\title{
Task 9 - Centrifugal Membrane Filtration
}

\author{
Semi-Annual Report
}

April 1 - September 30, 1996

Work Performed Under Contract No.: DE-FC21-94MC31388

RECEIVED

By

Daniel J. Stepan

MAR 311997

Thomas A. Moe

OSTI

For

U.S. Department of Energy

Office of Environmental Management

Office of Technology Development

1000 Independence Avenue

Washington, DC 20585
U.S. Department of Energy

Office of Fossil Energy

Morgantown Energy Technology Center

P.O. Box 880

Morgantown, West Virginia 26507-0880

DISTRIBUTION QF THIS DOCUMENT IS UNLIMHED

Energy and Environmental Research Center

University of North Dakota

P. O. Box 9018

Grand Forks, North Dakota 58202-9018

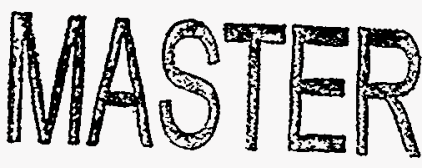




\section{Disclaimer}

This report was prepared as an account of work sponsored by an agency of the United States Government. Neither the United States Government nor any agency thereof, nor any of their employees, makes any warranty, express or implied, or assumes any legal liability or responsibility for the accuracy, completeness, or usefulness of any information, apparatus, product, or process disclosed, or represents that its use would not infringe privately owned rights. Reference herein to any specific commercial product, process, or service by trade name, trademark, manufacturer, or otherwise does not necessarily constitute or imply its endorsement, recommendation, or favoring by the United States Government or any agency thereof. The views and opinions of authors expressed herein do not necessarily state or reflect those of the United States Government or any agency thereof. 


\section{DISCLAIMIER}

Portions of this document may be illegible in electronic image products. Images are produced from the best available original document. 
LIST OF FIGURES $\ldots \ldots \ldots \ldots \ldots \ldots \ldots \ldots \ldots \ldots \ldots \ldots \ldots \ldots \ldots \ldots \ldots \ldots \ldots$

LIST OF TABLES $\ldots \ldots \ldots \ldots \ldots \ldots \ldots \ldots \ldots \ldots \ldots \ldots \ldots \ldots \ldots \ldots \ldots \ldots$ iii

EXECUTIVE SUMMARY $\ldots \ldots \ldots \ldots \ldots \ldots \ldots \ldots \ldots \ldots \ldots$ iv

1.0 INTRODUCTION $\ldots \ldots \ldots \ldots \ldots \ldots \ldots \ldots \ldots \ldots \ldots \ldots \ldots \ldots \ldots \ldots$

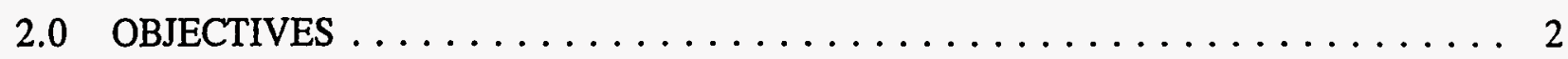

3.0 SPINTEK CENTRIFUGAL MEMBRANE FILTRATION $\ldots \ldots \ldots \ldots \ldots \ldots$

4.0 SPINTEK CENTRIFUGAL MEMBRANE FILTRATION TESTING PROGRAM $\ldots \ldots .5$

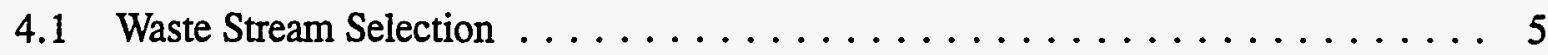

4.2 Membrane Screening and Selection $\ldots \ldots \ldots \ldots \ldots \ldots \ldots \ldots$

4.3 Centrifugal Membrane Filtration Performance Evaluation $\ldots \ldots \ldots \ldots \ldots 10$

4.3.1 Experimental Design $\ldots \ldots \ldots \ldots \ldots \ldots \ldots \ldots \ldots \ldots \ldots \ldots \ldots \ldots$

4.3.2 Statistical Analysis of Data . . . . . . . . . . . . . . 11

4.3.3 Surface Plots for Predictive Equation $\ldots \ldots \ldots \ldots \ldots \ldots \ldots \ldots \ldots$

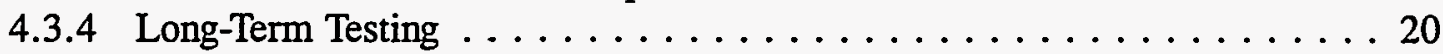

4.4 Preliminary Evaluation of Turbulence Promoters $\ldots \ldots \ldots \ldots \ldots \ldots \ldots 21$

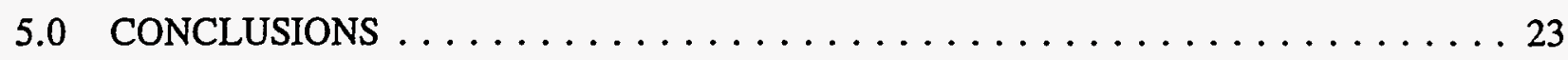

INTERIM PROJECT REPORT $\ldots \ldots \ldots \ldots \ldots \ldots \ldots \ldots \ldots \ldots \ldots \ldots$ Appendix A

MEMBRANE SCREENING TEST DATA $\ldots \ldots \ldots \ldots \ldots \ldots \ldots \ldots$ Appendix B

PERFORMANCE EVALUATION TEST DATA $\ldots \ldots \ldots \ldots \ldots \ldots$ Appendix C 


\section{LIST OF FIGURES}

1 Schematic representation of the SpinTek centrifugal membrane filtration system showing a

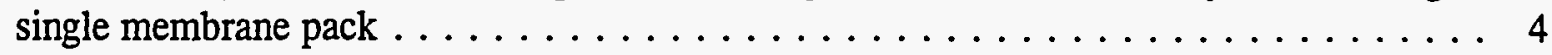

2 Schematic representation of SpinTek applications in the Tank Waste Focus Area $\ldots . \ldots 7$

3 SpinTek ST-IIL process schematic $\ldots \ldots \ldots \ldots \ldots \ldots \ldots \ldots \ldots \ldots \ldots \ldots \ldots$

4 Standard deviation as a function of flux $\ldots \ldots \ldots \ldots \ldots \ldots \ldots \ldots \ldots \ldots$

5 Constant variance criteria transformation for statistical model $\ldots \ldots \ldots \ldots \ldots \ldots$

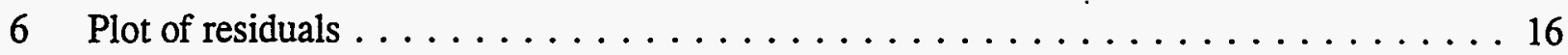

7. Log of measured flux versus $\log$ of predicted flux $\ldots \ldots \ldots \ldots \ldots \ldots$

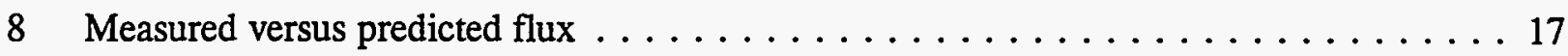

9 Predicted flux response surface - constant temperature, pressure, and time $\ldots \ldots \ldots 18$

10 Predicted flux response surface - constant temperature, rotor speed, and time $\ldots \ldots \ldots 18$

11 Predicted flux response surface - constant temperature, solids, and time $\ldots \ldots \ldots \ldots 19$

12 Predicted flux response surface - constant pressure, rotor speed, and time $\ldots \ldots \ldots 19$

13 Predicted flux response surface - constant temperature, rotor speed, and pressure . . . . 20

14 Permeate flux and modeling data as a function of time $\ldots \ldots \ldots \ldots \ldots \ldots \ldots$ 


\section{LIST OF TABLES}

1 Membranes Available for the SpinTek Centrifugal Membrane Filtration System _ . . . . 6

2 Surrogate Tank Waste Composition $\ldots \ldots \ldots \ldots \ldots \ldots$

3 Membrane Screening Results - Surrogate Tank Waste Solution with No Added Solids . . 9

4 Membrane Screening Results - Surrogate Tank Waste Solution with 3 wt\% Kaolin Added . . . . . . . . . . . . . . . . . . . . . 10

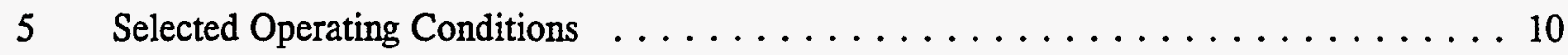

6 Test Conditions and Measured Data from Experimental Test Matrix $\ldots \ldots \ldots 12$

7 Summary of Model Statistics for Predicting the Logarithm of Permeate Flux . . . . . . 14

8 Membrane Composition at the Completion of Testing $\ldots \ldots \ldots \ldots \ldots \ldots$ 


\section{CENTRIFUGAL MEMBRANE FILTRATION}

\section{EXECUTIVE SUMMARY}

The Energy \& Environmental Research Center (EERC) has teamed with SpinTek Membrane Systems, Inc., the developer of a centrifugal membrane filtration technology, to demonstrate applications for the SpinTek technology within the U.S. Department of Energy (DOE) Environmental Management (EM) Program. 'The EERC is providing both assessment opportunities based on available information on wastes in the DOE inventory and focused testing to demonstrate the effectiveness of the SpinTek technology on selected waste streams. The technology uses supported microporous membranes rotating at high rpm, under pressure, to separate suspended and colloidal solids from liquid streams, yielding a solids-free permeate stream and a highly concentrated solids stream. This is a crosscutting technology that falls under the Efficient Separations and Processing Crosscutting Program, with potential application to tank wastes, contaminated groundwater, landfill leachate, and secondary liquid waste streams from other remediation processes, including decontamination and decommissioning systems. Based on the EERC's review of potential applications, the Tank Waste Focus Area was chosen for focused study.

Membrane-screening tests were performed with the SpinTek STC-X4 static test cell filtration unit, using five ceramic membranes with different pore size and composition. Based on permeate flux, a $0.25-\mu \mathrm{m} \mathrm{TiO}_{2} / \mathrm{Al}_{2} \mathrm{O}_{3}$ membrane was selected for detailed performance evaluation using the SpinTek ST-IIL centrifugal membrane filtration unit with a surrogate tank waste solution.

The performance of the ST-IIL centrifugal membrane filtration unit was evaluated using a statistical test design that determined the effect of four key operating parameters (temperature, pressure, membrane rotational speed, and solids loading) on permeate flux. All four variables were found to be statistically significant, with the magnitude of the effect in the order of temperature, solids loading, rotor speed, and pressure. Temperature, rotor speed, and pressure had an increasing effect on flux with increasing value, while increases in solids loading showed a decrease in permeate flux. Significant interactions between rotor speed and solids loading and pressure and solids loading were also observed. The regression equation derived from the test data had a correlation coefficient $\left(\mathrm{R}^{2}\right)$ of 0.934 , which represents a useful predictive capability for integrating the SpinTek technology into DOE cleanup efforts. An extended test run of $100 \mathrm{hr}$ performed on a surrogate tank waste solution showed some deterioration in filtration performance, based on flux, apparently due to the buildup of solids near the inner portion of the membrane where relative membrane velocities were low. Continued testing of the system will focus on modifications to the shear pattern across the entire membrane surface to affect improved long-term performance. 


\section{CENTRIFUGAL MEMBRANE FILTRATION}

\subsection{INTRODUCTION}

Approximately $90 \%$ of all the mixed, hazardous, and radioactive waste that has ever been generated in the United States has come from U.S. Department of Energy (DOE) installations. To compound the problem, the wastes were generated during the Cold War, when the race to develop nuclear weapons did not factor in environmental considerations. Environmental disposal was common and was believed to have limited long-term implications; however, many of the chemical constituents in the wastes are now known or suspected to pose significant human and environmental health concerns and are regulated under federal and state statutes. Fifty years of nuclear armament have produced spent nuclear fuel, more than 3000 inactive waste sites, an estimated $3.1 \mathrm{MM} \mathrm{m}^{3}$ of buried waste, deteriorating high-level nuclear waste storage tanks, and a public questioning DOE's commitment and ability to correct the problems. The total cost of environmental compliance and cleanup at the $18 \mathrm{DOE}$ facilities are estimated at approximately $\$ 60$ to $\$ 90$ billion $(1,2)$.

Recognizing the severity of environmental problems at its facilities, DOE has begun to remediate its contaminated sites. As part of these remediation efforts, basic research has been funded to improve the understanding of contaminant transport and fate and develop new and more appropriate remediation options.

The extent and complexity of contamination vary among the DOE sites according to the facility mission, size, and waste management practices. Even though many waste sites have been identified, the extent and complexity of the contamination are still largely unknown. The larger DOE facilities typically had multiple activities, which were chemically intensive, producing subsurface contamination of an extremely complex nature. The smaller facilities were more limited in their activities, resulting in less complex subsurface contamination. Contamination levels at the evaluated sites range from low levels of parts per billion $(\mathrm{ppb})$ for certain chemicals to elevated levels, as high as parts per hundred (pph), for other constituents.

Environmental media are contaminated with radionuclides, metals, and anions. Other contaminants include chlorinated solvents, fuel hydrocarbons, plasticizers, polychlorinated biphenyls (PCBs), explosives, organic acids, pesticides, and pharmaceutical wastes.

Concentrations of radionuclides in groundwater have been reported in the range of picocuries per liter (pCi/L) up to millicuries per liter $(\mathrm{mCi} / \mathrm{L})$.

The DOE Environmental Management (EM) Program, formerly known as the Office of Environmental Restoration and Waste Management, was created in November 1989 to initiate the management and remediation of contamination problems at several DOE facilities used in the research and production of nuclear arms and fuels over a period of nearly 50 years. These facilities contain 3700 specific sites with over 26,000 acres of hazardous or radioactive contaminated soils, groundwater, and structures. The EM mission is to minimize risks to the environment, human health, and safety posed by the production, handling, treatment, storage, transportation, and disposal of DOE waste and to bring DOE sites into compliance with all environmental regulations by the year 2019 . 
Within the EM Program is the Office of Science and Technology, formerly called the Office of Technology Development, which is responsible for developing technologies to meet DOE's 30-year goal for environmental restoration. This program is designed to identify and develop innovative and cost-effective technologies that can clean up the inventory of DOE nuclear component-manufacturing sites and manage DOE-generated waste faster, safer, and more costeffectively than is possible with current environmental cleanup technologies.

To achieve the EM objectives, there are five main focus areas within the EM Program, which are divided into several subprograms. These main focus areas are as follows:

- Contaminant Plumes Containment and Remediation

- Mixed-Waste Characterization, Treatment, and Disposal

- Radioactive Tank Waste Remediation

- Landfill Stabilization

- Decontamination and Decommissioning

The subprograms of these focus areas are in place to further concentrate similar efforts and promote and develop promising technologies. Together with private, federal, and state facilities, the EM Program hopes to provide visibility, focus, and accountability for DOE's waste management and cleanup efforts.

SpinTek Membrane Systems, Inc., has developed a novel centrifugal membrane filtration process that has application in remediation efforts currently under way at the various contaminated DOE sites. The greatest benefits of centrifugal membrane ultrafiltration include its efficient separations, efficient processing, and high-rate volume reduction capabilities. The innovative membrane filtration system coupled with centrifugal force gives this crosscutting technology distinct advantages over conventional ultrafiltration processes. It can accept feed streams containing relatively large particulate sizes, unlike hollow fiber ultrafilters, and can maintain high permeate flux at significantly higher feed solids concentrations than tubular or spirally wound ultrafiltration units. Rotating membrane ultrafiltration could be one step in a series of remediation operations or employed in series using multiple membrane types to selectively remove various waste components. Used as a stand-alone unit, it is capable of producing a permeate free of colloidal and suspended solids and a low-volume concentrate stream. The SpinTek technology may also be used as a complement to other cleanup efforts.

\subsection{OBJECTIVES}

This project consists of several integrated research phases related to the applicability, continued development, demonstration, and commercialization of the SpinTek centrifugal membrane filtration process for the remediation of liquid mixed-waste streams at DOE facilities. Specific Phase I project objectives include the following:

- A problem and opportunity assessment to identify applicable waste streams, including mixed wastes, associated with DOE sites 
- Development of detailed process data that will provide information on the application of the technology at DOE sites

- Testing and evaluation of a laboratory centrifugal membrane filtration unit using surrogate waste streams under a variety of operating conditions

- Development of process data that will allow optimization of the technology for appropriate DOE waste stream remediation

\subsection{SPINTEK CENTRIFUGAL MEMBRANE FILTRATION}

The SpinTek centrifugal membrane filtration process is designed to accomplish difficult separations under harsh conditions in a cost-effective manner. Using ultrafiltration membranes, the process separates suspended solids down to about 1000 molecular weight (MW). With reverseosmosis membranes, suspended solids smaller than $1000 \mathrm{MW}$ can be removed along with $96 \%-98 \%$ of dissolved solids. General applications for the system range from removing undesirable contaminants to concentration for recovery of solids or dissolved species. The feed stream to the filtration system is divided into two output streams, the permeate and the concentrate, with the permeate making up about $75 \%-95 \%$ of the effluent. Either the permeate or concentrate can be the desired end product, depending upon the application.

During ultrafiltration or reverse osmosis, liquid is forced through a semipermeable membrane, leaving suspended and dissolved solids larger than the nominal membrane pore size at the membrane interface. Therefore, the concentration of solids at the boundary between the membrane and the feed solution is increased. This phenomenon is referred to as concentration polarization, and the result is decreased flow through the membrane because of fouling. Traditional systems attempt to solve the problem of concentration polarization through the use of turbulence promoters or high recycle flow rates to remix the concentrated solution. However, these methods lose effectiveness with significant increases in solids concentration.

Research on concentration polarization phenomenon revealed that water flow and salt rejection both increased when a reverse-osmosis membrane was operated while centrifugal acceleration was applied parallel to the membrane-liquid interface. The SpinTek system takes advantage of these process dynamics. Disk-shaped membrane packs are stacked on a hollow rotor inside of a cylindrical housing. The rotor spins at speeds of up to $3500 \mathrm{rpm}$ to develop centrifugal forces exceeding 400 times the force of gravity across the membrane surfaces. Static turbulence promoters are located on either side of each membrane pack to impart shear forces to the concentrated boundary layer. Feed water enters through the cylindrical housing and is forced to flow across the filters. A back-pressure regulator/control valve is used to provide up to $150 \mathrm{psig}$ inside the pressure vessel, which is the driving force for the movement of product water through the membranes. Permeate is forced to the central hollow rotary shaft, which acts as the main permeate collector, and flows out of the membrane housing. Salts and solids left concentrated at the membrane surface are kept in suspension through turbulent shear and are swept to the perimeter of the disks by centrifugal force. The concentrate stream then exits the membrane housing and can be collected separately or recycled for additional concentration. A schematic of the basic SpinTek 
centrifugal membrane filtration system, showing a cutaway of a single membrane pack, is given in Figure 1.

Each membrane pack consists of a rigid internal support plate, with microfiltration membranes covering both sides. The membranes are separated from the support plate by a highly permeable layer of polypropylene mesh or similar type of material. Either membrane type can be used, depending on system design criteria. Since the membranes are flat, high-tech construction materials such as metal and ceramics may be used, as well as traditional cellulosic and polymeric formulations. Essentially, the system can be configured to utilize any commercially available flatsheet membrane. The membrane packs are stacked together to provide high packing density. The capacity of the unit depends on the number of packs and the height of the pressure vessel. Capacity can be increased or decreased by adding or subtracting packs.

The system operates well in high-fouling environments that would be unsuitable for conventional membrane systems operated in a static mode. To date, successful on-site testing has been performed on fermentation and food-processing streams and on process streams containing clays, colloids, and industrial oils. Specific examples of SpinTek system successes include recovery of latex from a dilute waste stream (1\% solids) and concentration up to $50 \%$; concentration of hydrated alumina to $40 \%$; yeast concentration above $35 \%$; and $90+\%$ recovery of protein from cheese whey. The centrifugal membrane filtration system has also been shown to be effective in concentrating nonsettleable solids to viscosities above 6400 centipoise.

SpinTek's rotating membrane is superior to traditional units because of its low fouling tendencies and increased flow characteristics. However, the membrane species used will require

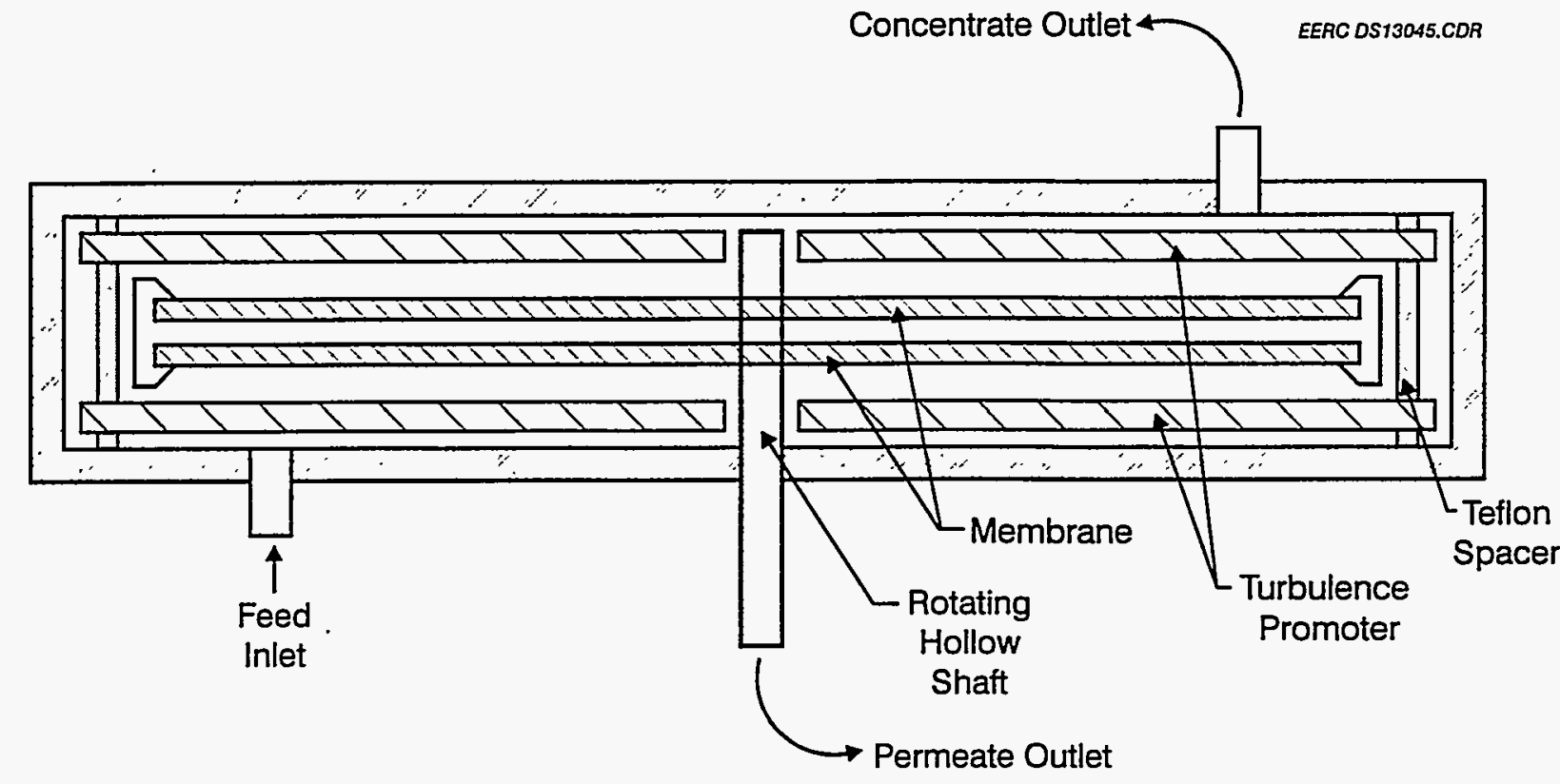

Figure 1. Schematic representation of the SpinTek centrifugal membrane filtration system showing a single membrane pack. 
evaluation for each waste stream to optimize product water flux. A wide range of membrane types can be utilized in the SpinTek system, with both hydrophilic and hydrophobic polymeric membrane types available, as well as membranes that show excellent resistance to solvents. Table 1 lists the various types of membranes that have been tested in the SpinTek system. These membranes are durable, industrial-grade membranes with unique characteristics that make them more or less suitable for various applications. Brief descriptions of each membrane's properties are included in the table.

\subsection{SPINTEK CENTRIFUGAL MEMBRANE FILTRATION TESTING PROGRAM}

\subsection{Waste Stream Selection}

A literature review was conducted to assess the liquid waste stream problem at DOE facilities and to identify potential applications of the SpinTek technology. An interim project report was prepared and is included as Appendix A. Based on that review, centrifugal membrane filtration was classified as a crosscutting technology that has potential application in all five DOE EM Program focus areas. For this study, the Radioactive Tank Waste Remediation Focus Area was selected for SpinTek testing and evaluation.

There are 332 underground storage tanks across the DOE complex accounting for 100 million gallons of radioactive wastes. The composition of the tank wastes varies, but typically contains nitrate and nitrate salts, hydrated metal oxides, phosphate precipitates, ferrocyanides, and radionuclides, such as ${ }^{90} \mathrm{Sr}$ and ${ }^{137} \mathrm{Cs}$. These wastes exist as three physical forms, both individually and in combination: liquid supernatant, sludge, and hard salt cake.

The SpinTek centrifugal membrane filtration process has several potential applications for the remediation of tank wastes, providing an effective means of waste separation and waste volume reduction, while producing a solids-free effluent that will improve the operation and efficiency of downstream unit operations for cesium and strontium removal. Figure 2 shows a schematic representation of these applications. The upper portion of the Figure 2 diagram illustrates the direct filtration of colloidal and suspended solids from tank waste supernatants, while the lower portion of the figure depicts its use in dewatering and solids concentration in conjunction with tank sludge-washing operations. Additionally, the SpinTek process could be used in conjunction with salt cake removal operations, such as "confined sluicing" where the permeate stream could be recycled back to the sluicing system, reducing the need for additional makeup water.

Table 2 provides a list of the surrogate chemicals and their respective concentrations that were used to simulate tank wastes during the centrifugal membrane filtration test trials. This particular solution represents the major contaminants in INEL (Idaho National Engineering Laboratory) high-sodium tank wastes.

In order to simulate the solids or sludge from tank wastes, a suspension of kaolin was added to the surrogate solution. This application of SpinTek centrifugal membrane filtration, then, was designed to demonstrate the efficient removal of suspended and colloidal solids in the supernatant, as well as solids separation during sludge and salt cake removal and washing operations. 


\section{TABLE 1}

Membranes Available for the SpinTek Centrifugal Membrane Filtration System

\author{
Polyvinylidene Fluoride \\ (PVDF)
}

Polysulfone (PS)

Polyethersulfone (PES)

Hydrophilic Polyethersulfone (HPES)

Polyester (PETE)

Polycarbonate (PC)

Polyolefin (PO)

Polyimide (PI)

Polyaramide (PA)
Excellent general-purpose membrane available in a variety of pore sizes that generally exhibits very good clean water fluxes. Resists biological attack; moderate $\mathrm{pH}$ and temperature limitations; moderately hydrophobic.

Good general-purpose membrane with good clean water fluxes and in some cases greater chemical resistance than PVDF. Resists biological attack; moderate $\mathrm{pH}$ and temperature limitations; moderately hydrophilic.

Exhibits many of the same characteristics as the PS membrane but has greater chemical resistance. Resistant to higher levels of oxidizing agents such as chlorine, certain aliphatic hydrocarbons, and alcohol.

Membrane specially formulated to be highly hydrophilic. Should only be used when normal PS or PES membranes perform marginally.

Track-etched membrane that is hydrophilic and has excellent chemical resistance to most commonly used chemicals. The most acid-resistant of commonly used membranes, but susceptible to attack by strong bases.

Track-etched membrane with poorer solvent resistance than PETE. Free of extractables and available in a wide range of pore sizes.

Very hydrophilic and most suited to oily water separations. More stringent temperature limitations with respect to most other polymeric membranes. Available pore sizes limited to the ultrafiltration range.

Very solvent-resistant, but available only in limited pore sizes. Should be considered in many hydrocarbon and solvent applications.

Excellent solvent resistance. However, permeate rates are generally lower than several other membrane types. Available pore sizes are limited to the ultrafiltration range.

continued . . 
TABLE 1 (continued)

Membranes Available for the SpinTek Centrifugal Membrane Filtration System

\begin{tabular}{ll}
\hline Teflon (PTFE) & $\begin{array}{l}\text { Excellent clean water fluxes and chemical resistance. } \\
\text { Current membrane formulations are very hydrophobic, } \\
\text { with more hydrophilic versions to be available in the } \\
\text { future. Weak mechanical strength makes membrane highly } \\
\text { susceptible to damage. Therefore, PTFE should be } \\
\text { selected only when its exceptional chemical resistance } \\
\text { overrides the structural shortcomings. }\end{array}$ \\
Ceramic (CR) & $\begin{array}{l}\text { Available in formulations with exceptional resistance to } \\
\text { solvents and temperature extremes. Very rugged and less } \\
\text { susceptible to irreversible fouling because of the range of } \\
\text { cleaning compounds that can be utilized for flux recovery. }\end{array}$ \\
Stainless Steel (SS) & $\begin{array}{l}\text { Sintered } 316 \text { SS construction. Chemically resistant with } \\
\text { extremely high clean water fluxes. Impervious to } \\
\text { biological attack, with excellent pH and temperature } \\
\text { resistance. Membrane has a tendency to trap particles } \\
\text { within the metal matrix and hence lose flux quickly. }\end{array}$ \\
\hline
\end{tabular}

EERC DS12518.CDR
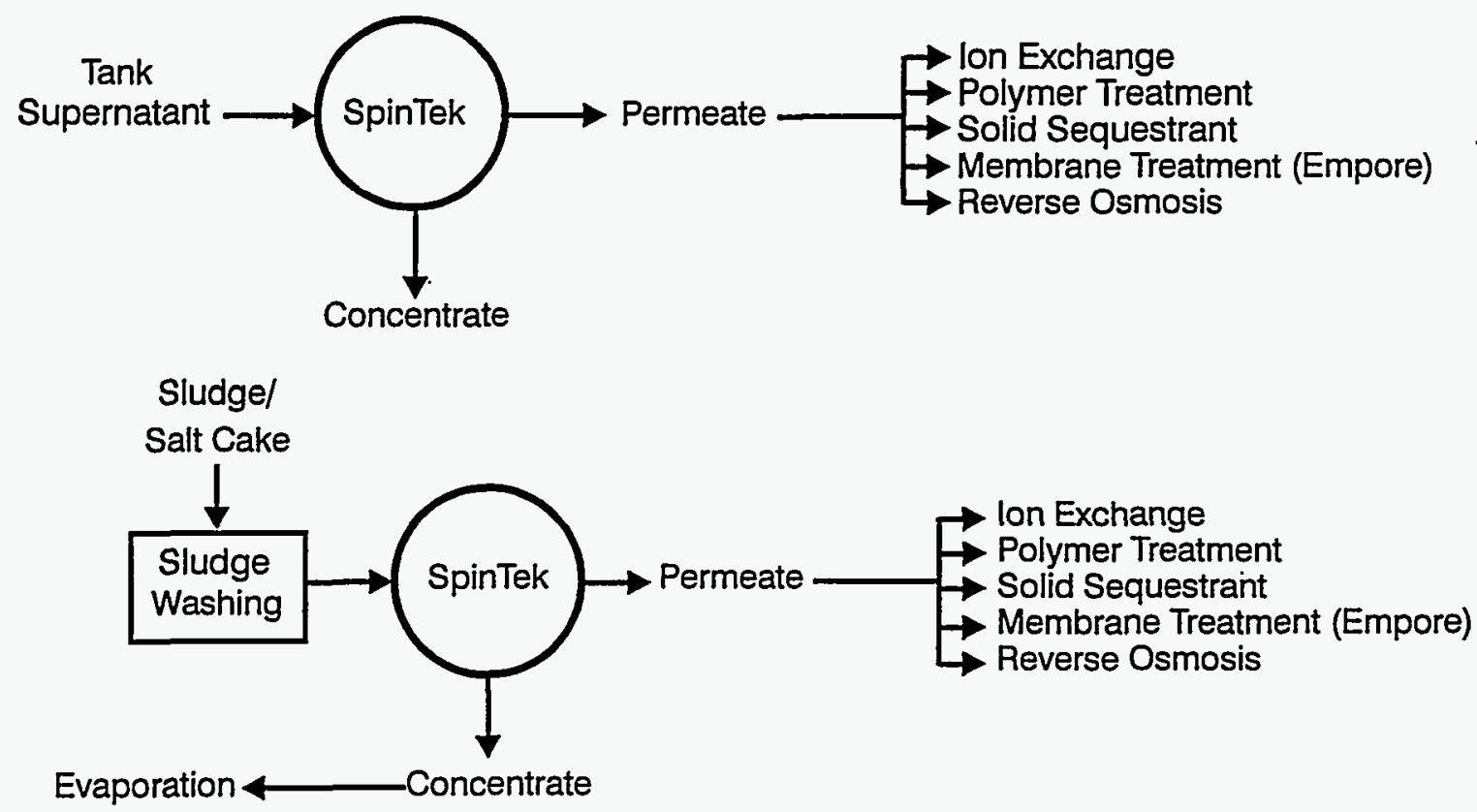

Figure 2. Schematic representation of SpinTek applications in the Tank Waste Focus Area. 
TABLE 2

Surrogate Tank Waste Composition

\begin{tabular}{lc}
\hline Component & Concentration, mol/L \\
\hline $\mathrm{NaNO}_{3}$ & 1.78 \\
$\mathrm{HNO}_{3}$ & 1.66 \\
$\mathrm{KNO}_{3}$ & 0.23 \\
$\mathrm{Ca}\left(\mathrm{NO}_{3}\right)_{2}$ & 0.04 \\
$\mathrm{H}_{2} \mathrm{SO}_{4}$ & 0.03 \\
$\mathrm{Fe}\left(\mathrm{NO}_{3}\right)_{3}$ & 0.03 \\
$\mathrm{H}_{3} \mathrm{BO}_{3}$ & 0.02 \\
$\mathrm{HCl}$ & 0.02 \\
$\mathrm{H}_{3} \mathrm{PO}_{4}$ & 0.01 \\
$\mathrm{Mn}\left(\mathrm{NO}_{3}\right)_{2}$ & 0.01 \\
$\mathrm{Al}\left(\mathrm{NO}_{3}\right)_{3}$ & 0.004 \\
$\mathrm{Cd}\left(\mathrm{NO}_{3}\right)_{2}$ & 0.002 \\
$\mathrm{Ni}\left(\mathrm{NO}_{3}\right)_{2}$ & 0.002 \\
$\mathrm{Hg}\left(\mathrm{NO}_{3}\right)_{2}$ & 0.002 \\
$\mathrm{CsNO}$ & 0.00004 \\
$\mathrm{Sr}\left(\mathrm{NO}_{3}\right)_{2}$ & 0.0000029 \\
\hline
\end{tabular}

\subsection{Membrane Screening and Selection}

SpinTek has a small test unit available with the capability to test up to four membrane types simultaneously at the same conditions on a given feed stream. This test unit, designated the STC-X4, makes use of static membrane cells in which the feed material flows past small sections of membrane (approximately 3 in. $\times 4.5$ in.) at velocities of up to about 15 fps. Feed material enters each cell and flows through a channel in a serpentine path to the cell exit. The flow channel is 0.312 in. wide and $0.100 \mathrm{in}$. deep. The total membrane area in which filtration takes place is $0.0493 \mathrm{ft}^{2}$. Using the STC-X4, many different membrane types, such as those listed in Table 1, can be quickly evaluated in terms of permeate flux, permeate quality, and membrane durability. Any flat-sheet membrane material available can be utilized. The results of STC-X4 testing can then be used to scale up to SpinTek's ST-IIL rotating membrane test unit.

Prior to testing and performance evaluation using the ST-IIL centrifugal membrane filtration unit, membrane-screening trials were conducted using SpinTek's STC-X4 test unit. Because of the nature of the tank wastes, ceramic membranes were considered for screening. Five ceramic membranes having different mean pore sizes and different compositions were evaluated during the screening trials:

- $0.25-\mu \mathrm{m} \mathrm{TiO}_{2} / \mathrm{Al}_{2} \mathrm{O}_{3}$

- $0.25-\mu \mathrm{m} \mathrm{TiO}_{2} / \mathrm{SiO}_{2}$

- $0.3-\mu \mathrm{m} \mathrm{ZrO}_{2} / \mathrm{TiO}_{2}$

- $0.4-\mu \mathrm{m} \mathrm{TiO}_{2} / \mathrm{Al}_{2} \mathrm{O}_{3}$

- $0.6-\mu \mathrm{m} \mathrm{ZrO}{ }_{2}$ 
Membrane-screening activities involved testing of the various membranes under several different operating conditions of pressure and solids loading. Temperature and flow rates were held constant throughout all screening tests at $90^{\circ} \mathrm{F}$ and 1 gallon per minute, respectively. Feed pressures were varied between 30 and 75 psig. Two different solids loadings, 0 and $3 \mathrm{wt} \%$, were evaluated.

Table 3 summarizes screening test results using the surrogate tank waste solution with no solids added. In general, all five different membranes exhibited an increase in flux with increasing feed pressure. Additionally, membranes with a larger nominal pore size had a greater flux.

The second set of membrane-screening trials was conducted using the surrogate tank waste solution with $3 \mathrm{wt} \%$ kaolin added. Table 4 summarizes test results with the $3 \mathrm{wt} \%$ surrogate tank waste solution at an average feed pressure of 29.5 psig. Performance, as measured by flux, was observed to be higher than test trials under similar pressure conditions with no added solids. The reason for this is not clearly understood, as one would intuitively think that additional solids would reduce flux. It is noteworthy, however, that flux did not show an increase with increasing nominal pore size, apparently due to plugging of the larger diameter pores, thus reducing overall filtration capacity. Test data collected during membrane screening and selection activities are presented in Appendix B.

Based on a review of the data by SpinTek and EERC personnel, it was decided that a smaller diameter pore size membrane would be used for performance evaluation using the SpinTek ST-IIL unit. A $0.25-\mu \mathrm{m} \mathrm{TiO}_{2} / \mathrm{Al}_{2} \mathrm{O}_{3}$ ceramic membrane was ultimately selected because of performance and availability.

\section{TABLE 3}

Membrane Screening Results - Surrogate Tank Waste Solution with No Added Solids

\begin{tabular}{|c|c|c|c|c|c|}
\hline $\begin{array}{l}\text { Membrane: } \\
\text { Pore Size: }\end{array}$ & $\begin{array}{c}\mathrm{TiO}_{2} / \mathrm{Al}_{2} \mathrm{O}_{3} \\
0.25 \mu \mathrm{m} \\
\end{array}$ & $\begin{array}{l}\mathrm{TiO}_{2} / \mathrm{SiO}_{2} \\
0.25 \mu \mathrm{m}\end{array}$ & $\begin{array}{c}\mathrm{ZrO}_{2} / \mathrm{TiO}_{2} \\
0.3 \mu \mathrm{m} \\
\end{array}$ & $\begin{array}{c}\mathrm{TiO}_{2} / \mathrm{Al}_{2} \mathrm{O}_{3} \\
0.4 \mu \mathrm{m} \\
\end{array}$ & $\begin{array}{c}\mathrm{ZrO} \\
0.6 \mu \mathrm{m} \\
.\end{array}$ \\
\hline Average Feed Pressure, psig & 28.4 & 28.4 & 28.4 & 28.5 & 28.5 \\
\hline Average Concentrate Pressure, psig & 6.6 & 6.6 & 6.6 & 5.6 & 5.6 \\
\hline Average Measured Flux, gal $/ \mathrm{ft}^{2}$-day & 63.8 & 69.0 & 63.1 & 57.1 & 60.7 \\
\hline Average Feed Pressure, psig & 46.9 & 46.9 & 46.9 & $-^{a}$ & - \\
\hline Average Concentrate Pressure, psig & 14.6 & 14.6 & 14.6 & - & - \\
\hline Average Measured Flux, gal $/ \mathrm{ft}^{2}$-day & 72.3 & 75.0 & 82.0 & - & - \\
\hline Average Feed Pressure, psig & 72.5 & 72.5 & 72.5 & 72.9 & 72.9 \\
\hline Average Concentrate Pressure, psig & 39.9 & 39.9 & 39.9 & 39.1 & 39.1 \\
\hline Average Measured Flux, gal $/ \mathrm{ft}^{2}$-day & 143.3 & 145.6 & 149.3 & 200.1 & 207.6 \\
\hline
\end{tabular}

a Not performed. 


\section{TABLE 4}

Membrane Screening Results - Surrogate Tank Waste Solution with 3 wt\% Kaolin Added

\begin{tabular}{|c|c|c|c|c|c|}
\hline $\begin{array}{l}\text { Membrane: } \\
\text { Pore Size: }\end{array}$ & $\begin{array}{c}\mathrm{TiO}_{2} / \mathrm{Al}_{2} \mathrm{O}_{3} \\
0.25 \mu \mathrm{m}\end{array}$ & $\begin{array}{c}\mathrm{TiO}_{2} / \mathrm{SiO}_{2} \\
0.25 \mu \mathrm{m}\end{array}$ & $\begin{array}{c}\mathrm{ZrO}_{2} / \mathrm{TiO}_{2} \\
0.3 \mu \mathrm{m}\end{array}$ & $\begin{array}{c}\mathrm{TiO}_{2} / \mathrm{Al}_{2} \mathrm{O}_{3} \\
0.4 \mu \mathrm{m}\end{array}$ & $\begin{array}{c}\mathrm{ZrO}_{2} \\
0.6 \mu \mathrm{m}\end{array}$ \\
\hline Average Feed Pressure, psig & 29.9 & 29.9 & 29.9 & 29.2 & 29.2 \\
\hline Average Concentrate Pressure, psig & 7.0 & 7.0 & 7.0 & 6.0 & 6.0 \\
\hline Average Measured Flux, gal/ft'-day & 100.0 & 104.9 & 96.3 & 82.3 & 79.0 \\
\hline
\end{tabular}

\subsection{Centrifugal Membrane Filtration Performance Evaluation}

Performance evaluation of the centrifugal membrane filtration process was conducted using the $0.25-\mu \mathrm{m} \mathrm{TiO} / \mathrm{Al}_{2} \mathrm{O}_{3}$ ceramic membrane on the SpinTek ST-IIL unit. Based upon preliminary process evaluation, four key factors-temperature, pressure, rotor speed, and solids loading-were identified as having the greatest potential influence on process performance, as measured by flux. An experimental test plan was designed to account for statistically significant interactions of the variables and the determination of optimized operating conditions for treating tank wastes. Table 5 shows the coded design set point operating conditions selected to determine process performance and develop statistical data. Based on the optimized operating condition, an extended run was conducted to determine performance over time. The following discusses the methodology and results of this testing.

A schematic diagram of the ST-IIL process, as configured for testing and evaluation, is shown in Figure 3. The surrogate tank waste solution for a designated test was placed in the surge tank, and the mixer was turned on to maintain the solids in suspension. The feed pump was turned on, and flow was established at 132 gallons per hr for each test run. The pressure controller was then set to the desired pressure for the run. When the desired pressure was obtained, the rotor motor was turned on and set to the desired $\mathrm{rpm}$. The system was then allowed to equilibrate at the desired temperature setting. Each experimental run was conducted over a 3- to 4-hr period after set point operating conditions had been established. When steady-state flux was achieved, data were collected over a 2 -hr period. Operating data for each of the 27 test runs are provided in Appendix C.

TABLE 5

Selected Operating Conditions

\begin{tabular}{lccc}
\hline & \multicolumn{3}{c}{ Test Condition Set Point } \\
Parameter & Lower $(-1)$ & Midpoint $(0)$ & Upper $(+1)$ \\
\hline Temperature, ${ }^{\circ} \mathrm{F}$ & 60 & 90 & 120 \\
Pressure, psig & 45 & 60 & 75 \\
Rotor Speed, rpm & 1000 & 1500 & 2000 \\
Solids Loading, wt\% & 1 & 15 & 30 \\
\hline
\end{tabular}




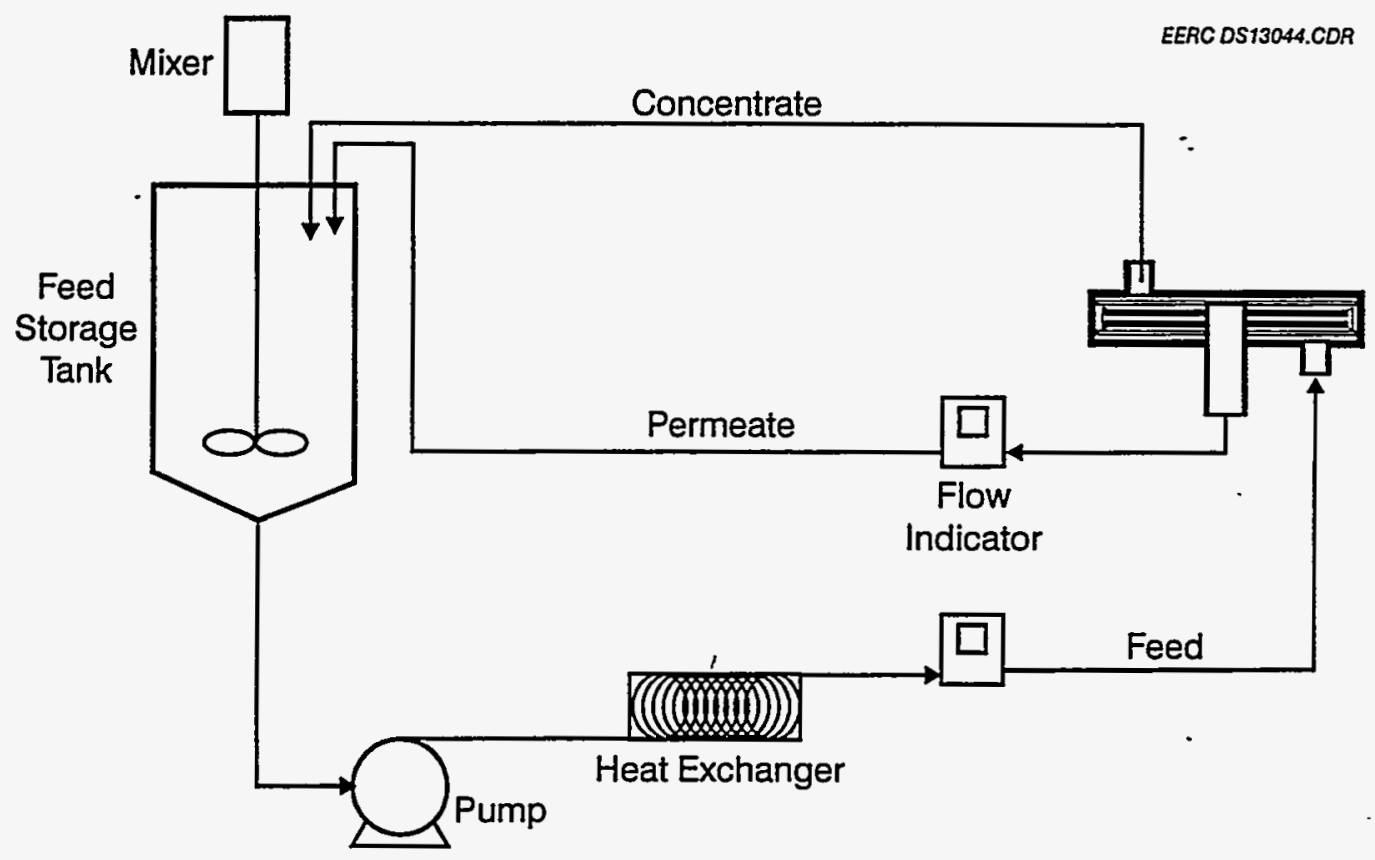

Figure 3. SpinTek ST-IIL process schematic.

\subsubsection{Experimental Design}

In order to determine the effects of temperature, feed pressure, rotor speed, and solids concentration, a statistically designed experimental matrix was created that would test the significance of these variables and also result in an empirical model of the observed response. The simplest design is a full-factorial matrix that requires approximately 20 experiments to formulate a linear model. However, a response surface model using a Box-Behnken design requires only 27 experiments, but affords all of the benefits of a quadratic model to fit the experimental data. The latter design is more efficient in the event that one of the experimental variables results in a nonlinear response and was used for constructing the test matrix used in this study. The advantage of using a statistical design is that the levels of the variables are chosen to maintain the independence of their effects on the response or, alternatively speaking, the variables are uncorrelated with each other.

\subsubsection{Statistical Analysis of Data}

The actual uncoded independent and dependent variables are summarized in Table 6 for each of the 27 tests performed using the Box-Behnken design. Note that the experiments were randomized prior to execution to remove the effect of bias from the analysis, and the time reflects the cumulative run time based on the randomized order. The average steady-state data shown in Table 6 were modeled using the multiple linear regression procedure (REG) from the SAS/STAT ${ }^{\mathrm{TM}}$ statistical software package to determine the significant variables and regression coefficients. As part of the analysis, all independent variables were coded to normalize the range of values between -1 and 1 . The normalization of the data allows one to examine the regression coefficients for their relative contribution to the model. 
TABLE 6

Test Conditions and Measured Data from Experimental Test Matrix

\begin{tabular}{rrrrrrrc}
\hline Test & $\begin{array}{c}\text { Rotor Speed, } \\
\text { rpm }\end{array}$ & $\begin{array}{c}\text { Pressure, } \\
\text { psig }\end{array}$ & $\begin{array}{c}\text { Solids, } \\
\%\end{array}$ & $\begin{array}{r}\text { Temperature, } \\
{ }^{\circ} \text { F }\end{array}$ & $\begin{array}{r}\text { Flux, } \\
\text { gfd }\end{array}$ & $\begin{array}{r}\text { Std. Dev., } \\
\text { gfd }\end{array}$ & $\begin{array}{c}\text { Time, } \\
\text { hr }\end{array}$ \\
\hline 1 & 1991 & 44 & 15 & 90 & 316 & 14.4 & 3.32 \\
2 & 1501 & 75 & 15 & 119 & 427 & 16.3 & 5.65 \\
3 & 995 & 61 & 1 & 90 & 528 & 16.7 & 9.2 \\
4 & 1991 & 60 & 30 & 90 & 254 & 14.4 & 12.22 \\
5 & 986 & 45 & 15 & 90 & 193 & 4.0 & 15.14 \\
6 & 1498 & 74 & 1 & 89 & 488 & 11.0 & 19.12 \\
7 & 2008 & 61 & 1 & 89 & 526 & 12.2 & 22.64 \\
8 & 1491 & 60 & 30 & 119 & 259 & 15.2 & 25.11 \\
9 & 1501 & 47 & 30 & 89 & 184 & 7.3 & 28.29 \\
10 & 1500 & 62 & 1 & 120 & 604 & 20.8 & 31.47 \\
11 & 993 & 58 & 30 & 89 & 90 & 9.8 & 34.62 \\
12 & 1499 & 77 & 30 & 90 & 155 & 12.5 & 37.67 \\
13 & 1503 & 61 & 15 & 90 & 218 & 10.9 & 40.85 \\
14 & 998 & 59 & 15 & 120 & 221 & 10.3 & 44.07 \\
15 & 1499 & 61 & 30 & 59 & 114 & 11.5 & 48.09 \\
16 & 1512 & 59 & 15 & 90 & 209 & 8.9 & 51.11 \\
17 & 997 & 57 & 15 & 60 & 92 & 2.3 & 55.13 \\
18 & 995 & 78 & 15 & 89 & 160 & 3.3 & 58.7 \\
19 & 1499 & 59 & 15 & 90 & 200 & 10.5 & 61.13 \\
20 & 1501 & 43 & 15 & 60 & 114 & 1.1 & 63.53 \\
21 & 1498 & 59 & 1 & 61 & 140 & 1.8 & 66.03 \\
22 & 2015 & 73 & 15 & 90 & 329 & 18.2 & 69.1 \\
23 & 1994 & 63 & 15 & 120 & 408 & 23.2 & 72.38 \\
24 & 1507 & 47 & 15 & 120 & 299 & 12.5 & 75.7 \\
25 & 1501 & 71 & 15 & 60 & 162 & 5.4 & 78.45 \\
26 & 1487 & 45 & 1 & 89 & 193 & 5.7 & 81.07 \\
27 & 2005 & 57 & 15 & 60 & 180 & 3.5 & 84.05 \\
\hline & & & & & & &
\end{tabular}

In order to make value judgments on the significance of the variables in a model through statistical inference, certain criteria must be met. Among these are that the form of the model is correct, that the variance among the observations of the dependent variable (flux) are constant, and that the errors are normally distributed. These criteria can also be interrelated. For example, if the form of the model is incorrect or the variance of the observations is not a constant, then the errors may not be normally distributed. The objective of the modeling effort is, therefore, to meet these criteria by selecting the best form of the model by transforming the data.

Figure 4 shows a plot of the standard deviation as a function of the permeate flux (response variable). As mentioned previously, the variance of the data should be constant across all 


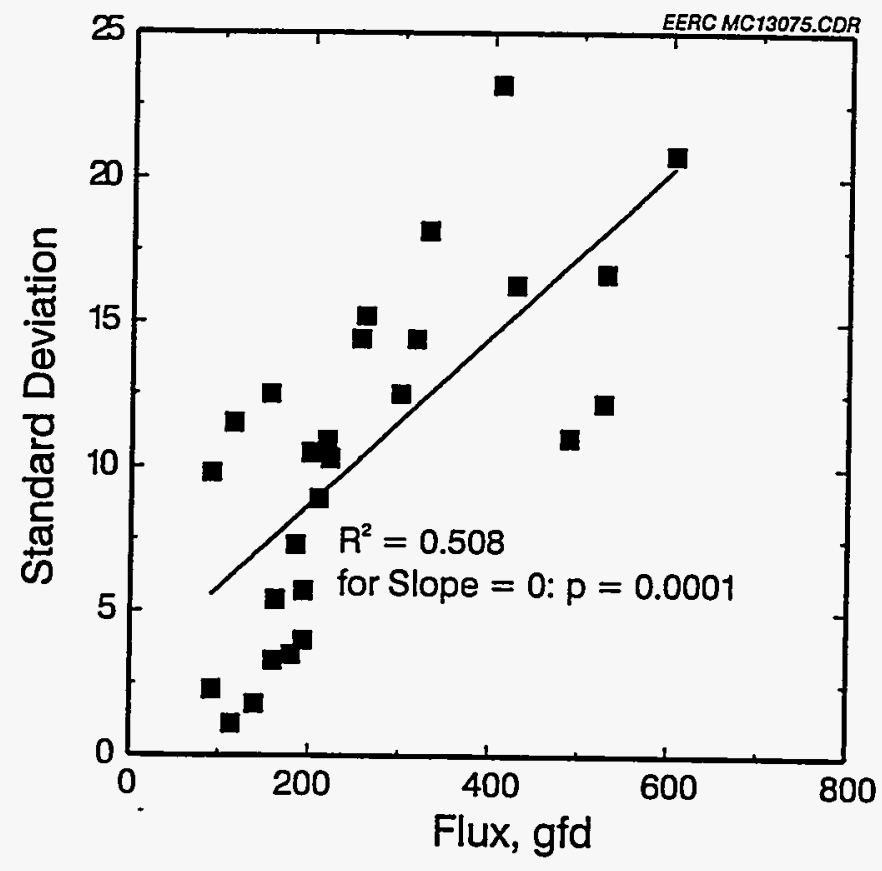

Figure 4. Standard deviation as a function of flux.

observations; however, the figure clearly shows a trend of increasing variance with flux rate. This trend was shown to be statistically significant, with only a 1 -in-10,000 chance of the slope of the line actually being zero. On the other hand, Figure 5 presents the relative standard deviation (RSD) as a function of the flux and shows the independence of the RSD with flux. The RSD is the standard deviation divided by the mean and puts the data variation on a percent, rather than an absolute, basis. In the case of Figure 5, there is a $29 \%$ probability that the slope is equal to zero; therefore, the slope is very likely to be zero.

To meet the criteria of constant variance as depicted in Figure 5, the data must be transformed. This can be accomplished by taking the logarithm of the response variable (flux); i.e., the standard deviation expressed on a log scale is equivalent to the RSD on a linear scale. Because of the logarithmic transformation, the resultant model will be exponential and of the form $y=a b^{x}$, where $a$ and $b$ are constants calculated from the linear regression.

A summary of the statistics for the best-fit linear regression model is presented in Table 7 . As seen from Table 7, all of the variables included in the test matrix were significant at $95 \%$ probability or better, and in addition, there were interactions among the parameters of rotor speed and solids concentration and feed pressure and solids concentration.

The magnitude of the parameter estimates (coefficients used to formulate the modeling equation) indicate that the influence of the variables follows the order:

Temperature $\geq$ Solids Concentration $>$ Rotor Speed $>$ Pressure 


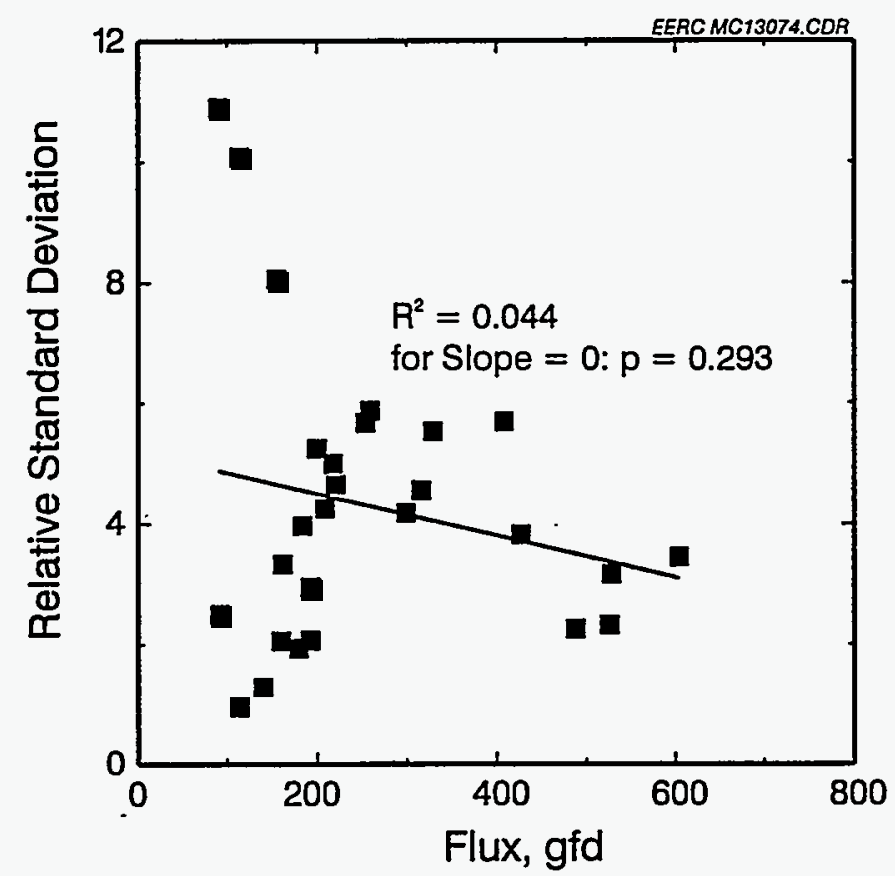

Figure 5. Constant variance criteria transformation for statistical model.

\section{TABLE 7}

Summary of Model Statistics for Predicting the Logarithm of Permeate Flux Dependent Variable: $\log$ (flux)

\begin{tabular}{lccccc}
\hline & \multicolumn{5}{c}{ Parameter Estimates } \\
\cline { 2 - 6 } Variable & DF & $\begin{array}{c}\text { Parameter } \\
\text { Estimate }\end{array}$ & $\begin{array}{c}\text { Standard } \\
\text { Error }\end{array}$ & $\begin{array}{c}\text { T for H0 } \\
\text { Parameter }=0\end{array}$ & Prob. > $>|\mathrm{T}|$ \\
\hline Intercept & 1 & 2.356882 & 0.01346299 & 175.064 & 0.0001 \\
rpm & 1 & 0.144016 & 0.02073814 & 6.944 & 0.0001 \\
Pressure & 1 & 0.057948 & 0.02361105 & 2.454 & 0.0239 \\
Solids & 1 & -0.179544 & 0.02028288 & -8.852 & 0.0001 \\
Temperature & 1 & 0.180538 & 0.02185245 & 8.262 & 0.0001 \\
rpm $\times$ Solids & 1 & 0.090153 & 0.03619734 & 2.491 & 0.0222 \\
Pressure $\times$ Solids & 1 & -0.093287 & 0.04307829 & -2.166 & 0.0433 \\
Time & 1 & -0.103791 & 0.02498318 & -4.154 & 0.0005 \\
\hline
\end{tabular}

The sign of the coefficients also indicates that an increase in pressure, rotor speed, or temperature will result in an increase in the permeate flux, while increasing the solids concentration will tend to decrease the flux. The interaction terms show that the level of one variable in the interaction will impact the effect of the other; e.g., the change in the flux of the permeate with rpm will be different at a low solids concentration than at a high solids concentration. The cumulative run time variable was introduced into the model after discovering the membrane flux had 
diminished as a result of running the 27 experiments. This variable was found to be very significant, illustrating that other variables such as chemical or physical erosion or membrane plugging had occurred in a manner that was time-dependent.

Figure 6 shows a plot of the residuals, the difference between the actual values and those predicted by the statistically derived model, as a function of normal probability. The straight-line plot shows that the errors are normally distributed, required for applying statistical inference, and indicates that the logarithmic transformation of the data was adequate. The points at both ends of the line may be outliers to the data set and correspond to Tests 3 and 21 in the test matrix shown in Table 6. These two points share a common element of being at the lowest solids concentration of $1 \%$. If these data points are valid, then this could indicate that the flux is sensitive to the region near $0 \%$ solids concentration and that these concentrations need to be measured more accurately to be modeled correctly, e.g., $0.55 \%$ solids instead of $1 \%$.

Figure 7 presents a plot of the actual $\log (f l u x)$ versus the predicted $\log (f l u x)$ for the best-fit model shown in Table 7. The correlation coefficient $\left(R^{2}\right)$ for the predictive model was 0.934 , with the 45 degree line representing a perfect fit of an $R^{2}$ equal to 1 . An equivalent plot is shown in Figure 8 , where the inverse logarithms were used to convert the data to more meaningful units.

The predictive equation presented in Table 7 is in the coded form and is not readily solvable by direct substitution of values from Table 6 for the independent variables. After substituting in the independent-variable-coding equations, multiplying and collecting terms, the functional form of the modeling equation becomes:

$$
\begin{gathered}
\log (f l u x)=1.448+9.270 \mathrm{E}-5(\mathrm{rpm})+8.999 \mathrm{E}-3(\mathrm{P})-8.310 \mathrm{E}-3(\mathrm{~S})+5.918 \mathrm{E}-3(\mathrm{~T}) \quad \text { [Eq. 1] } \\
+1.208 \mathrm{E}-5(\mathrm{rpm})(\mathrm{S})-3.670 \mathrm{E}-4(\mathrm{P})(\mathrm{S})-2.572 \mathrm{E}-3(\mathrm{t})
\end{gathered}
$$

where

$$
\begin{aligned}
& \mathrm{rpm}=\text { rotor speed, } \mathrm{rpm} \\
& \mathrm{P}=\text { feed pressure, } \mathrm{psig} \\
& \mathrm{S}=\text { solids concentration, } \% \\
& \mathrm{~T}=\text { temperature, }{ }^{\circ} \mathrm{F} \\
& \mathrm{t}=\text { cumulative run time, } \mathrm{hr}
\end{aligned}
$$

Because all of the coefficients are small, further simplification by taking the inverse logarithm of Equation 1 will result in loss of accuracy unless the coefficients are carried out to 7 or more significant figures. The exponential form of Equation 1 then becomes

$$
\begin{aligned}
\text { Flux }= & 28.05(1.0002135)^{\mathrm{rm}}(1.02094)^{\mathrm{P}}(0.98105)^{\mathrm{S}}(1.01372)^{\mathrm{T}}(1.00002782)^{\mathrm{rpm} \cdot \mathrm{s}} \\
& (0.99916)^{\mathrm{PSS}}(0.99409)^{\mathrm{t}}
\end{aligned}
$$

Equation 2 allows for direct substitution of operating parameters into the exponents to calculate a flux rate, but Equation 1 is more accurate and practical to apply. It should be noted that extrapolation of this model beyond the limits of the experimental range should be avoided, since the results are unpredictable. 


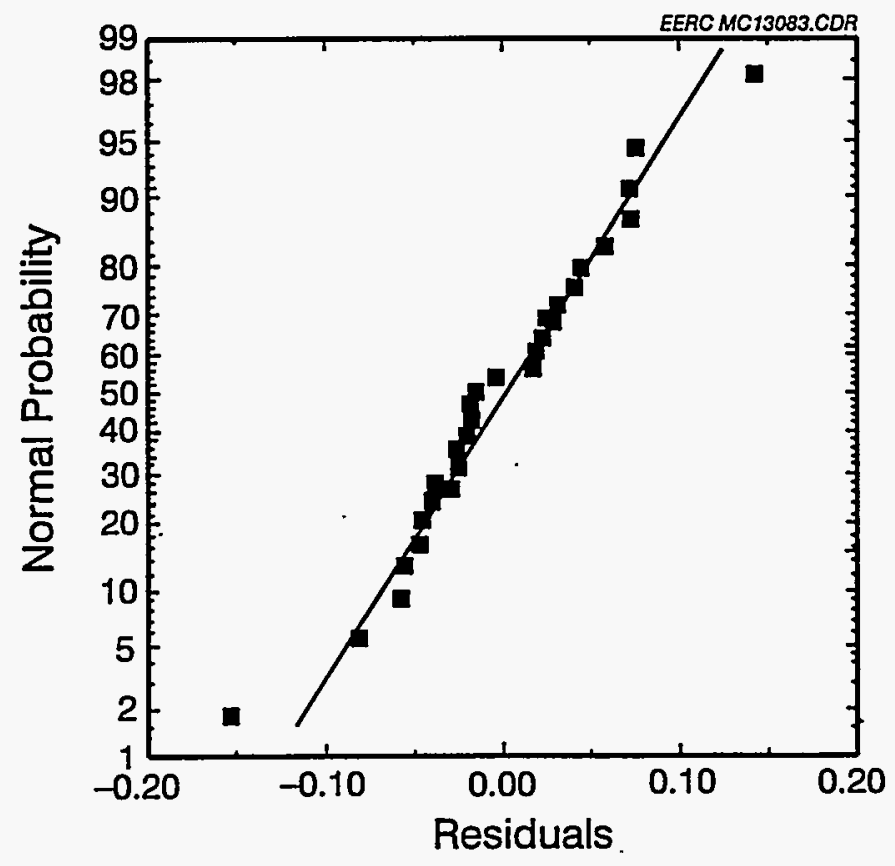

Figure 6. Plot of residuals.

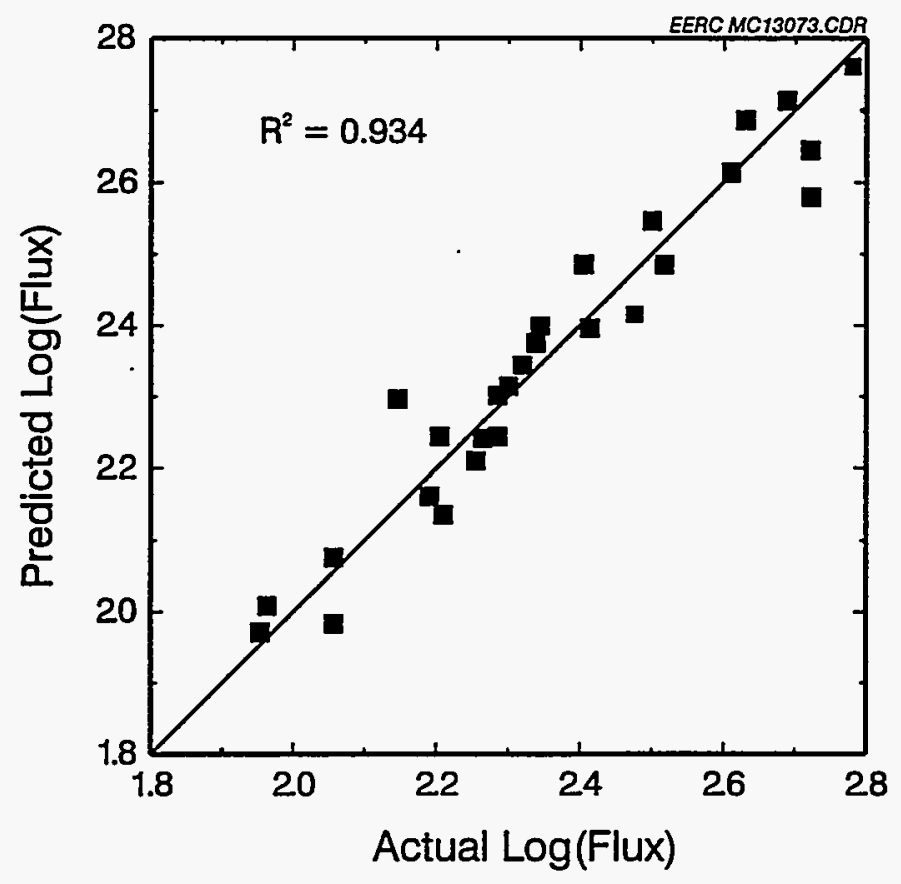

Figure 7. Log of measured flux versus log of predicted flux. 


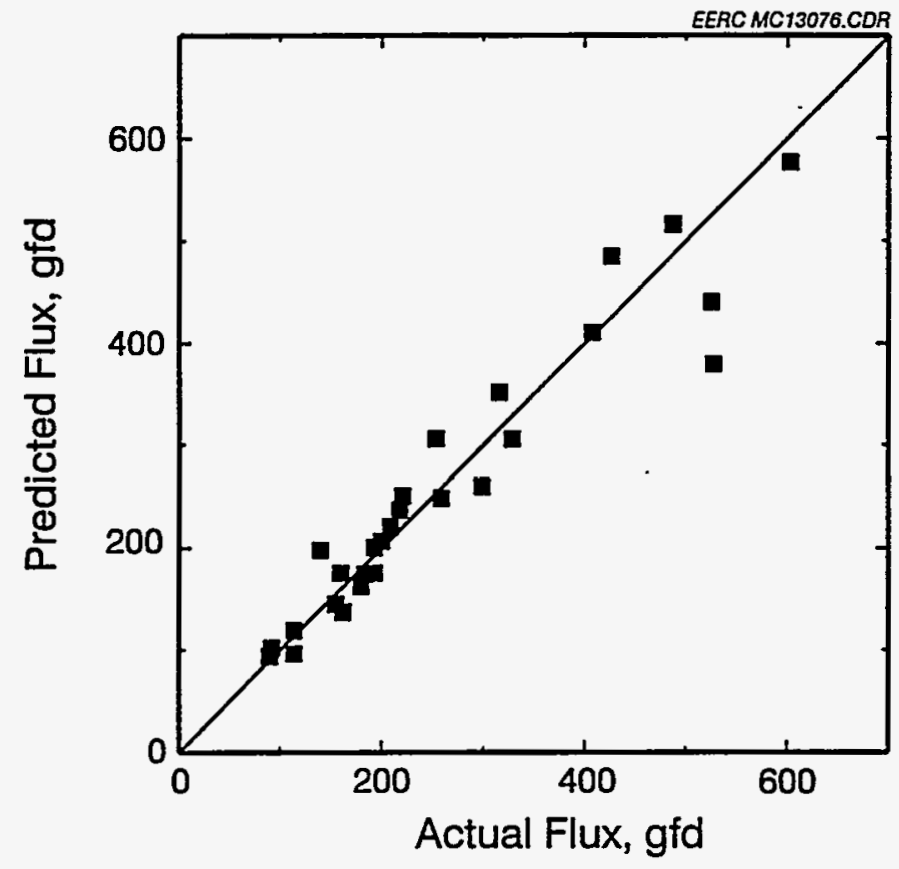

Figure 8. Measured versus predicted flux.

\subsubsection{Surface Plots for Predictive Equation}

Surface plots provide a convenient means of examining the model graphically. These plots were generated from the statistical model characterizing the experimental data. The coded version of Equation 2 was used to plot the response surfaces for the variables in the model because of greater accuracy and simplicity in solving the equation. In the coded format, $a+1$ is the greatest value of the experimental range, 0 is the midpoint, and -1 is the smallest value. For example, a nominal rotor speed of $2000 \mathrm{rpm}$ equals +1 , while $1000 \mathrm{rpm}$ is equal to -1 . Since there were six dimensions to the predictive equation, any three of these were held constant at the midpoint of the experimental range while the other variables were changed. Figures 9 through 13 present the plots for the prediction of flux as a function of the operating parameters.

Both rotor speed and feed pressure were shown in the model to interact with solids concentration. This effect can be seen in Figures 9 and 10 as a change in the slope of surfaces between parallel $x$ and $y$ axes, but is most evident in Figure 10, where the slope of the pressure effect on the flux actually changes sign as solids concentration is changed from $1 \%$ to $30 \%$ (-1 to +1 ). The remaining surface plots, Figures 11 through 13, present surfaces without interactions including, respectively, rotor speed versus pressure, temperature versus solids concentration, and time versus solids concentration. Note that the interaction terms drop out of the coded modeling equations when the variables are held constant at the midpoint values $(0)$. These figures show that the response to each of the modeling parameters ranges from nearly linear, under certain conditions such as in Figure 8, to that of significant curvature as shown in the other figures. 


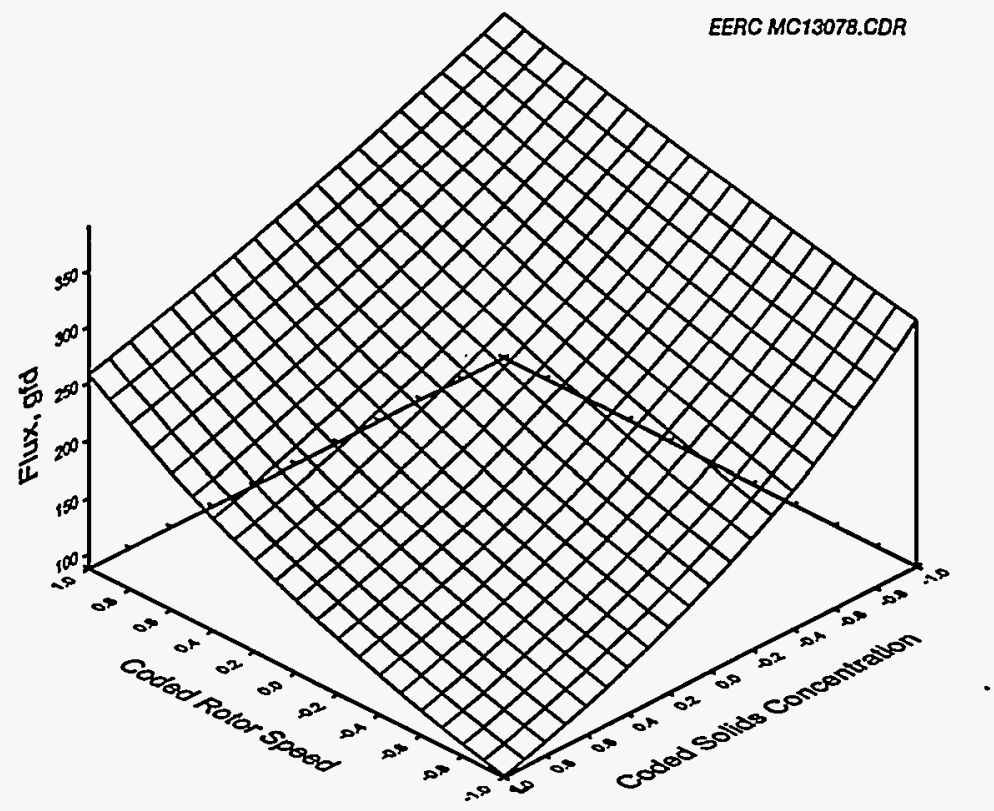

Figure 9. Predicted flux response surface - constant temperature, pressure, and time.

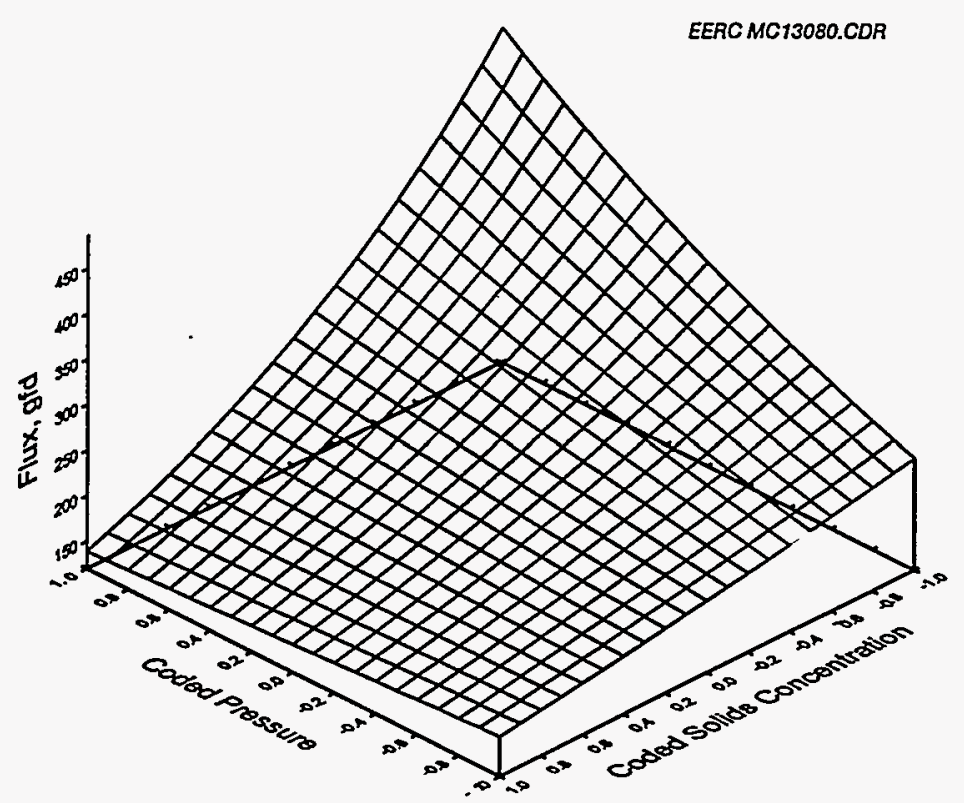

Figure 10. Predicted flux response surface - constant temperature, rotor speed, and time. 


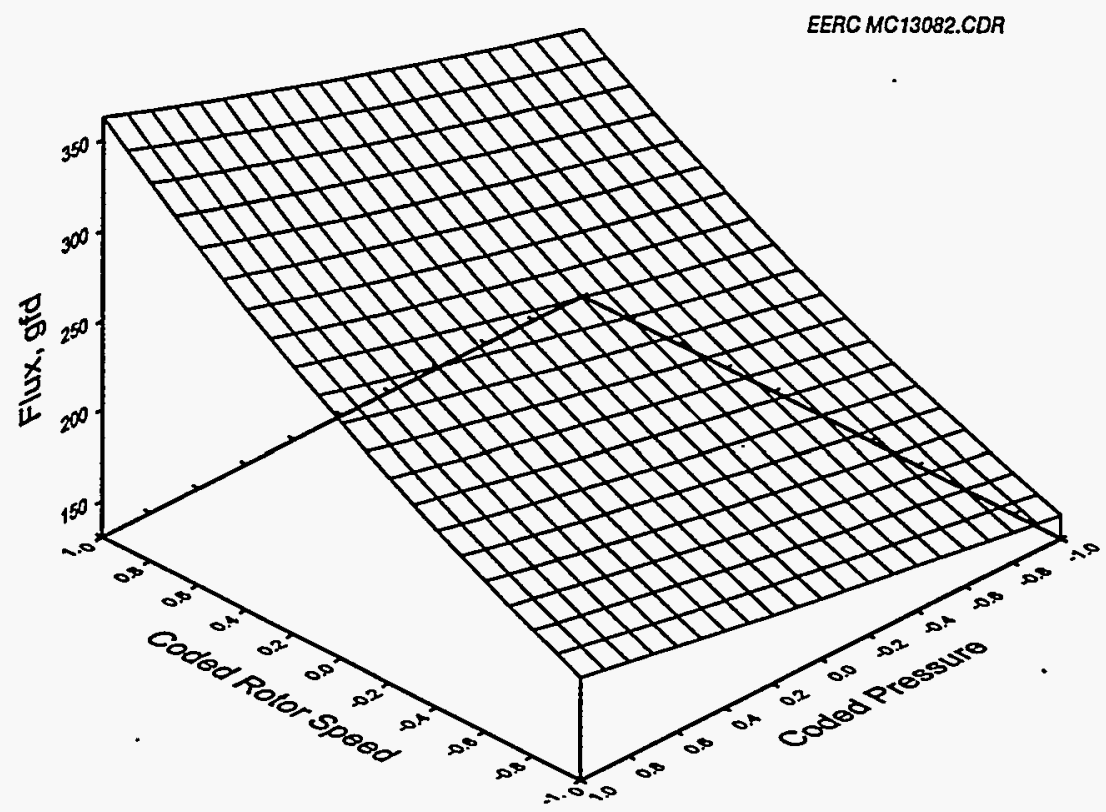

Figure 11. Predicted flux response surface - constant temperature, solids, and time.

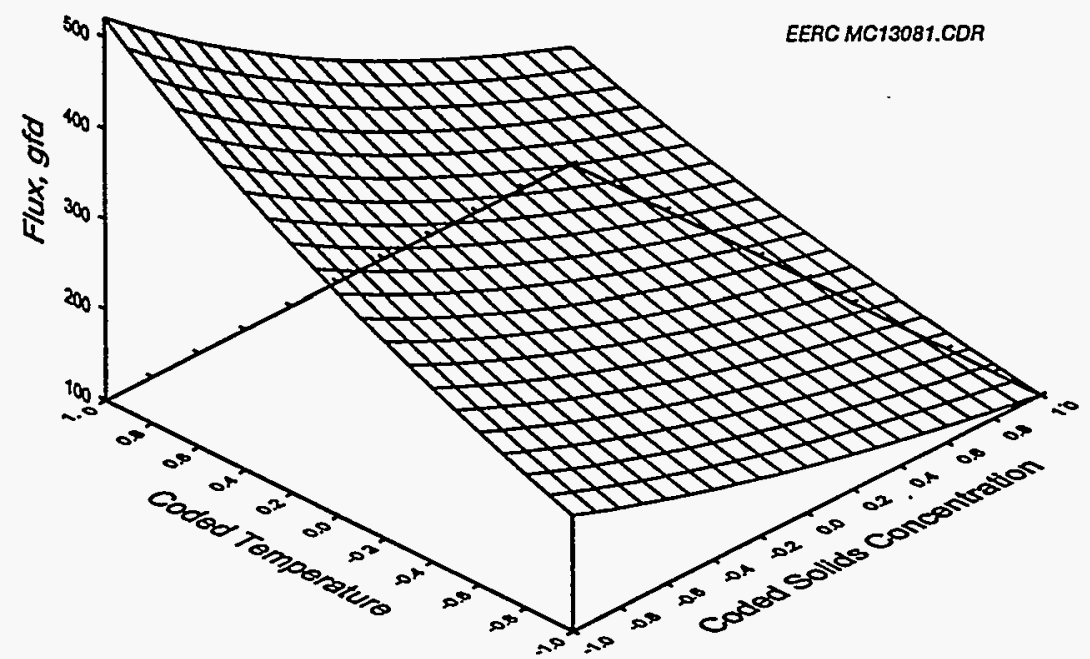

Figure 12. Predicted flux response surface - constant pressure, rotor speed, and time. 


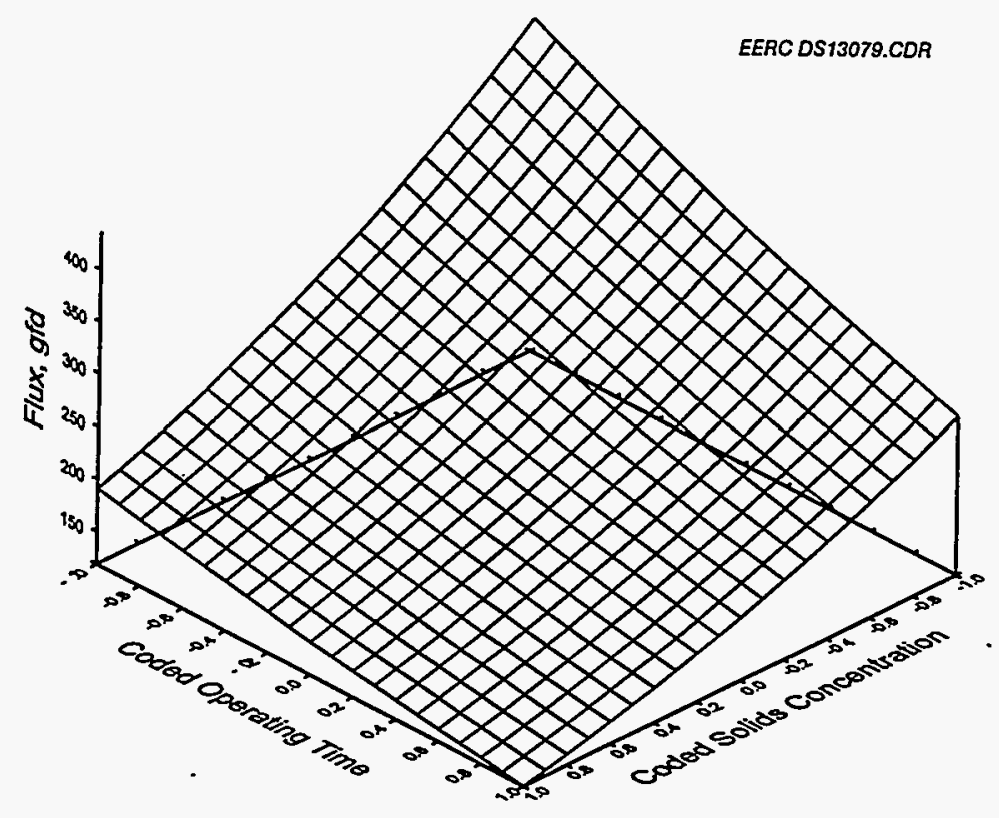

Figure 13. Predicted flux response surface - constant temperature, rotor speed, and pressure.

\subsubsection{Long-Term Testing}

After completion of the previous test matrix, the rotor assembly was fitted with new membranes to test their long-term (200-hr) performance for particle separation. The nominal conditions for the long-term test were as follows: $75 \mathrm{psig}$ feed pressure, $120^{\circ} \mathrm{F}$ feed temperature, $2000 \mathrm{rpm}$ rotor speed, and $15 \mathrm{wt} \%$ solids. The equipment was successfully operated for approximately $100 \mathrm{hr}$ before the run was terminated because of ancillary equipment failure. However, enough data were acquired to test the time dependency factor found to be very significant in the model.

Figure 14 presents the membrane permeate flux data and a variety of modeling data as a function of time. The flux data were acquired at 30 -min intervals and were logged as point measurements. The best fit of the flux data was found to be linear, with an $\mathrm{R}^{2}=0.848$, while an exponential fit followed with an $\mathrm{R}^{2}=0.830$ (these data are not shown in the figure). Even though the best fit was shown to be linear, more data would be required to confirm this conclusion, since one might expect the flux rate to approach zero asymptotically rather than linearly, or possibly the flux rate would level off and never reach zero. In any case, the exponential model derived from the experimental data is a very good approximation to this type of data.

The curves plotted in Figure 14 present various scenarios for application of this model to the data acquired for the new membranes. The line in this figure labeled "Model" and denoted by the dotted line is for the application of the derived model (Equation 1) to the long-term testing. The values for pressure, temperature, rotor speed, and solids concentration were held constant, with operating time being the only variable changed. In this case, the model overpredicts the permeate flux, but generally reflects the trend of the data. However, there is no reason to expect the new 


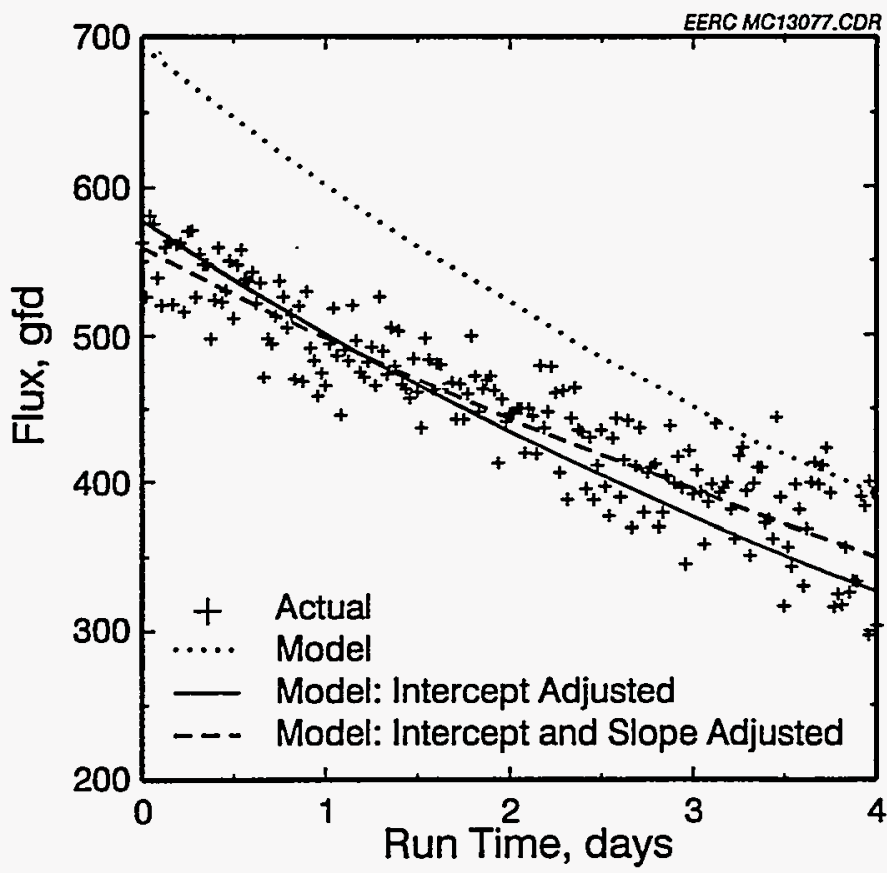

Figure 14. Permeate flux and modeling data as a function of time.

membranes to have identical baseline flux rates to the old set of membranes. For this reason, the intercept to the original model was adjusted from 1.448 to 1.369 in Equation 1 to produce the solidline curve, since the intercept dictates the baseline value for a given set of test parameters. The third curve in Figure 14, represented by the dashed line, shows the "best fit" that could be made if the slope of the time variable in the model (Equation 1) was shifted from -0.002572 to -0.002100 , and again, the intercept changed to a value of 1.355 to reflect the adjustment in baseline values.

The adjustment of the model's intercept to better reflect these data can be justified, especially since at this time no data exist on membrane variability. If this variability were determined, then the discrepancy between the model fit and the actual data could be better categorized as a chance versus a true difference in the membranes. Changing the slope of the time variable in Equation 1 is not justifiable, except to model the latter data set, since the original experimental test matrix was performed at a variety of run conditions that may also influence the time-dependent wearing of the membrane.

\subsection{Preliminary Evaluation of Turbulence Promoters}

Following each test run used to develop data for statistical analysis, the membranes were removed from the stationary housing, rinsed with tap water to remove any solids from the membrane surface, and inspected for wear and any damage. At the conclusion of both matrix testing and the extended run, membrane wear was noticeable. The original blue color of the titanium dioxide coating was worn from the perimeter of the membrane, approximately 1.5 inches from the outer edge. In order to investigate the wear or abrasion of the membrane coating, the composition of the membrane surface was analyzed by scanning electron microscopy (SEM). SEM analysis confirmed that the $\mathrm{TiO}_{2}$ coating had been abraded from the membrane surface, exposing the stainless steel support plate. Table 8 lists the normalized weight percent composition of selected 
oxides across the membrane surface, from the outside edge to the inner diameter of the membrane. The data in columns "Outside" and "Inside" were areas covered by the stainless steel retaining ring and were not directly exposed to the surrogate test solution or the fluid dynamics encountered by the other portions of the membrane. Titanium dioxide was the major surface component in the covered regions (outside and inside) and the inner portion of the membrane. However, in the areas where surface abrasion was visibly evident (outer and middle), the predominant metal oxide was iron. Chromium oxide was also present at higher levels in the regions of visible wear. These data indicate that the surface coating $\left(\mathrm{TiO}_{2} / \mathrm{Al}_{2} \mathrm{O}_{3}\right)$ had indeed been abraded from the surface, exposing the stainless steel support disk.

Several factors may contribute to the observed wear pattern. The first relates to a function of membrane velocity. The highest velocity occurs at the outside perimeter of the membrane, where the abrasion was most apparent. The second factor was the accumulation of a thin layer of clay solids that collected on the membrane surface near the inner portions of the membrane. This buildup of solids in this region prevents the scouring action observed near the outer perimeter.

The net effect on filtration performance due to membrane wear or solids buildup on the inner portions of the membrane surface is not known. However, based on the statistical analysis of operational data and data developed during the extended run, performance, as measured by permeate flux, decreased with time. It should be noted that permeate quality, as measured by

\section{TABLE 8}

Membrane Composition at the Completion of Testing

\begin{tabular}{lccccc}
\hline & \multicolumn{5}{c}{ Normalized Weight Percent } \\
\cline { 2 - 6 } Oxide & Outside & Outer & Middle & Inner & Inside \\
\hline $\mathrm{NaO}_{2}$ & & & & & \\
$\mathrm{Al}_{2} \mathrm{O}_{3}$ & 1.2 & 0.0 & 0.0 & 0.9 & 1.2 \\
$\mathrm{SiO}_{2}$ & 2.9 & 1.1 & 0.6 & 2.6 & 2.9 \\
$\mathrm{P}_{2} \mathrm{O}_{5}$ & 4.1 & 3.0 & 2.5 & 9.8 & 4.1 \\
$\mathrm{SO}_{3}$ & 0.2 & 0.0 & 0.4 & 0.2 & 0.2 \\
$\mathrm{ClO}$ & 0.2 & 1.1 & 1.2 & 0.0 & 0.2 \\
$\mathrm{~K}_{2} \mathrm{O}$ & 0.2 & 0.0 & 0.0 & 0.0 & 0.2 \\
$\mathrm{CaO}$ & 0.2 & 0.0 & 0.0 & 0.0 & 0.2 \\
$\mathrm{TiO}_{2}$ & 0.0 & 0.0 & 0.1 & 0.3 & 0.0 \\
$\mathrm{Cr}_{2} \mathrm{O}_{3}$ & 75.1 & 23.4 & 25.1 & 75.3 & 75.1 \\
$\mathrm{Fe}_{2} \mathrm{O}_{3}$ & 5.5 & 19.4 & 17.6 & 3.6 & 5.5 \\
\hline
\end{tabular}


turbidity and suspended solids, showed no deterioration throughout all testing, producing a turbidity-free permeate. Additionally, the observed wear is a membrane-related phenomena, and is not a system deficiency.

The ST-IIL system employs the use of "wagon wheel" turbulence promoters on either side of the membrane disk to create a shear force across the membrane surface to prevent the development of solids buildup. Based on membrane abrasion and solids buildup observed during testing, it appears that the current configuration of the turbulence promoters allows membrane cleaning where linear velocities are greater than approximately $17.5 \mathrm{fps}$. Optimization of the design of the turbulence promoter should allow the entire surface to be free of solids buildup, which should improve permeate flux. The effect of turbulence promoter optimization on membrane wear is not readily apparent at this time. However, under optimized turbulence conditions, it may be anticipated that higher rotational speeds would not be required to maintain self-cleaning membranes.

\subsection{CONCLUSIONS}

SpinTek centrifugal membrane filtration has applications in several program elements of the EM focus areas and as a crosscutting technology. Its unique ability in combining centrifugal force with membrane filtration allows for the continuous removal and concentration of selected contaminants from groundwater, surface water, process water, process chemicals, etc. The combination of the ceramic membrane with the low-fouling characteristics for the rotary system results in SpinTek's ability to concentrate hazardous wastewater to a slurry-type level and fractionate dilute low-level wastes, high-level wastes, and hazardous waste and reduce tank sludge. The system does not generate any secondary waste streams, and because it is self-contained, it reduces worker exposure and chance of spillage.

Rotating membrane filtration technology, such as the process developed by SpinTek Membrane Systems, Inc., appears to have great potential in remediation efforts currently under way at the various contaminated DOE sites. The greatest benefits of centrifugal membrane ultrafiltration include its efficient separations, efficient processing, and high-rate volume reduction capabilities. The innovative membrane filtration system coupled with centrifugal force gives this crosscutting technology distinct advantages over conventional ultrafiltration processes. It can accept feed streams containing relatively large particulate sizes, unlike hollow-fiber ultrafilters, and can maintain high permeate flux at significantly higher feed solids concentrations than tubular or spirally wound ultrafiltration units. Rotating membrane ultrafiltration could be one step in a series of remediation operations or employed in series using multiple membrane types to selectively remove various waste components. Used as a stand-alone unit, it is capable of producing a permeate free of colloidal and suspended solids and a low-volume concentrate stream. The Spin'Tek technology may also be used as a complement to other cleanup efforts.

Phase I research efforts have indicated that the SpinTek process is well suited for application to the cleanup of liquid mixed-waste streams at DOE facilities. The process configuration provides higher flux rates per unit membrane area in easily transportable, compact treatment units. The process is a near-tank technology that can be easily integrated with other unit operations to improve the operation and efficiency of downstream unit operations, such as adsorption and ion exchange. 
Filtration performance of the SpinTek process may be further enhanced through efforts to optimize the design of the turbulence promoters to allow the entire surface to be free of solids buildup, which should improve permeate flux. Optimization of the turbulence promoter design should be considered, followed by scaleup of the process to effectively interface with other treatment technologies in use at DOE facilities. 
Energy \&

Environmental

Research

Center

APPENDIX A

INTERIM PROJECT REPORT 
7 त 


\section{SPINTEK INTERIM PROJECT REPORT}

Prepared for:

Dr. Venkat Venkataraman

U.S. Department of Energy

Morgantown Energy Technology Center

PO Box 880, MS C05

Morgantwn, WV 26507-0880

Prepared by:

Rose H. Fraley Marc D. Kurz

Thomas A. Moe Steve S. Philbrick

Daniel J. Stepan

Energy \& Environmental Research Center University of North Dakota PO Box 9018

Grand Forks, ND 58202-9018

William A. Greene Patricia A. Kirk

SpinTek Membrane Systems Inc. 16421 Gothard Street, Unit A Huntington Beach, CA 92647 
Energy $\mathcal{E}$

Environmental

Research

Center

APPENDIX B

MEMBRANE SCREENING TEST DATA 
SpinTek Static Test Cell STC-X4

$-\cdot-\cdot 0.25$ um TiO2/Al2O3 - - - 0.25 um TiO2/SiO2 - - - 0.3 um ZrO2/TiO2

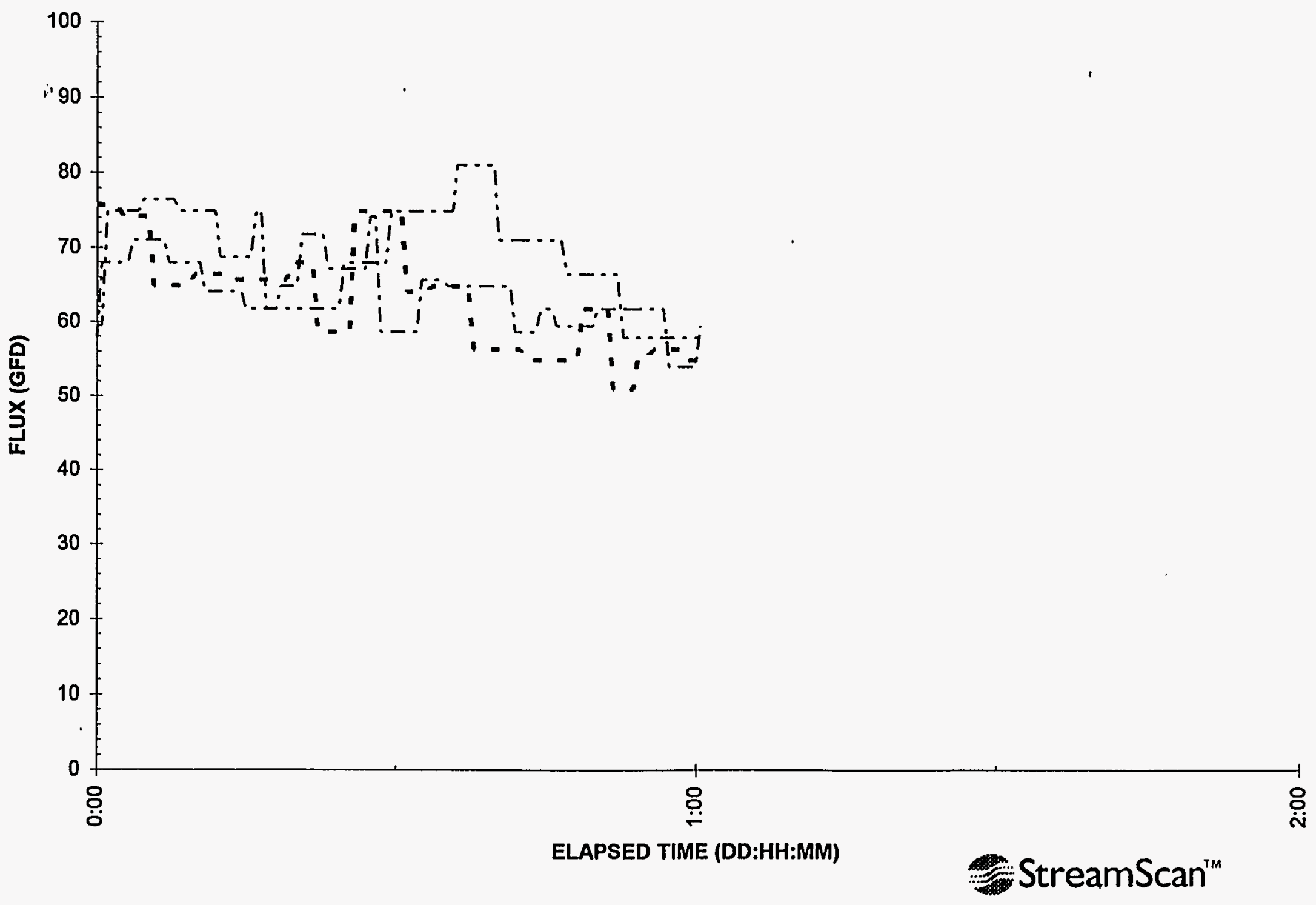




\begin{tabular}{|c|c|c|c|c|c|c|c|}
\hline \multicolumn{8}{|l|}{ SCRN1 } \\
\hline \multicolumn{8}{|c|}{0.25 um $\mathrm{TiO}_{2} / \mathrm{Al}_{2} \mathrm{O}_{3}$} \\
\hline $\begin{array}{c}\text { TIME OF } \\
\text { DAY }\end{array}$ & $\begin{array}{l}\text { ELAPSED } \\
\text { TIME }\end{array}$ & $\begin{array}{c}\text { FEED } \\
\text { PRESSURE }\end{array}$ & $\begin{array}{c}\text { CONC } \\
\text { PRESSURE }\end{array}$ & $\begin{array}{c}\text { FEED FLOW } \\
\text { (L/min) }\end{array}$ & FEED TEMP & $\begin{array}{l}\text { PERMEATE } \\
\text { FLOW }\end{array}$ & $\begin{array}{l}\text { PERMEATE } \\
\text { FLUX }\end{array}$ \\
\hline & (HH:MM) & (psig) & (psig) & (gpm) & $\left({ }^{\circ} \mathrm{F}\right)$ & (ml/min) & (gal $/ \mathrm{ft}^{2}-$ day) \\
\hline $14: 54$ & $0: 00$ & 29.0 & 7.0 & 0.8 & 89 & 7.5 & 58 \\
\hline $14: 55$ & $0: 00$ & 28.0 & 7.0 & 0.8 & 89 & 8.8 & 68 \\
\hline $14: 55$ & $0: 01$ & 32.0 & 7.0 & 0.9 & 90 & 8.8 & 68 \\
\hline $14: 56$ & 0:01 & 31.0 & 7.0 & 0.9 & 89 & 8.8 & 68 \\
\hline $14: 56$ & $0: 02$ & 31.0 & 7.0 & 0.9 & 90 & 8.8 & 68 \\
\hline $14: 57$ & $0: 02$ & 31.0 & 7.0 & 0.9 & 90 & 8.8 & 68 \\
\hline $14: 57$ & $0: 03$ & 31.0 & 7.0 & 0.9 & 89 & 8.8 & 68 \\
\hline $14: 58$ & $0: 03$ & 31.0 & 7.0 & 0.9 & 90 & 9.2 & 71 \\
\hline $14: 58$ & $0: 04$ & 31.0 & 7.0 & 0.9 & 89 & 9.2 & 71 \\
\hline $14: 59$ & $0: 04$ & 30.0 & 7.0 & 0.9 & 90 & 9.2 & 71 \\
\hline $14: 59$ & $0: 05$ & 27.0 & 6.0 & 0.8 & 90 & 9.2 & 71 \\
\hline $15: 00$ & $0: 05$ & 27.0 & 6.0 & 0.8 & 89 & 9.2 & 71 \\
\hline $15: 00$ & $0: 06$ & 27.0 & 6.0 & 0.8 & 90 & 9.2 & 71 \\
\hline 15:01 & $0: 06$ & 30.0 & 7.0 & 0.8 & 89 & 9.2 & 71 \\
\hline $15: 01$ & $0: 07$ & 30.0 & 7.0 & 0.9 & 90 & 8.8 & 68 \\
\hline $15: 02$ & $0: 07$ & 30.0 & 7.0 & 0.8 & 90 & 8.8 & 68 \\
\hline $15: 02$ & $0: 08$ & 28.0 & 7.0 & 0.8 & 89 & 8.8 & 68 \\
\hline $15: 03$ & $0: 08$ & 28.0 & 7.0 & 0.8 & 90 & 8.8 & 68 \\
\hline $15: 03$ & $0: 09$ & 27.0 & 7.0 & 0.8 & 89 & 8.8 & 68 \\
\hline 15:04 & $0: 09$ & 27.0 & 6.0 & 0.8 & 89 & 8.8 & 68 \\
\hline $15: 04$ & $0: 10$ & 27.0 & 7.0 & 0.8 & 90 & 8.8 & 68 \\
\hline $15: 05$ & $0: 10$ & 27.0 & 7.0 & 0.8 & 89 & 8.3 & 64 \\
\hline $15: 05$ & $0: 11$ & 27.0 & 6.0 & 0.8 & 90 & 8.3 & 64 \\
\hline $15: 06$ & $0: 11$ & 27.0 & 6.0 & 0.8 & 90 & 8.3 & 64 \\
\hline $15: 06$ & $0: 12$ & 27.0 & 6.0 & 0.8 & 90 & 8.3 & 64 \\
\hline 15:07 & $0: 12$ & 27.0 & 6.0 & 0.8 & 90 & 8.3 & 64 \\
\hline $15: 07$ & $0: 13$ & 27.0 & 6.0 & 0.8 & 89 . & 8.3 & 64 \\
\hline $15: 08$ & $0: 13$ & 27.0 & 6.0 & 0.8 & 90 & 8.3 & 64 \\
\hline $15: 09$ & $0: 14$ & 27.0 & 6.0 & 0.8 & 89 & 8.3 & 64 \\
\hline $15: 09$ & $0: 14$ & 27.0 & 7.0 & 0.8 & 89 & 8.0 & 62 \\
\hline $15: 10$ & $0: 15$ & 30.0 & 7.0 & 0.8 & 90 & 8.0 & 62 \\
\hline $15: 10$ & $0: 15$ & 29.0 & 7.0 & 0.8 & 89 & 8.0 & 62 \\
\hline $15: 11$ & $0: 16$ & 29.0 & 7.0 & 0.8 & 90 & 8.0 & 62 \\
\hline $15: 11$ & $0: 16$ & 29.0 & 7.0 & 0.8 & 89 & 8.0 & 62 \\
\hline $15: 12$ & $0: 17$ & 29.0 & 7.0 & 0.8 & 89 & 8.0 & 62 \\
\hline $15: 12$ & $0: 18$ & 29.0 & 7.0 & 0.8 & 90 & 8.0 & 62 \\
\hline $15: 13$ & $0: 18$ & 29.0 & 7.0 & 0.8 & 89 & 8.4 & 65 \\
\hline $15: 13$ & $0: 19$ & 29.0 & 7.0 & 0.8 & 90 & 8.4 & 65 \\
\hline $15: 14$ & $0: 19$ & 29.0 & 7.0 & 0.8 & 89 & 8.4 & 65 \\
\hline $15: 14$ & $0: 20$ & 29.0 & 7.0 & 0.8 & 89 & 8.4 & 65 \\
\hline $15: 15$ & $0: 20$ & 29.0 & 7.0 & 0.8 & 90 & 9.3 & 72 \\
\hline $15: 15$ & $0: 21$ & 29.0 & 7.0 & 0.8 & 89 & 9.3 & 72 \\
\hline $15: 16$ & $0: 21$ & 29.0 & 7.0 & 0.8 & 90 & 9.3 & 72 \\
\hline $15: 16$ & $0: 22$ & 29.0 & 7.0 & 0.8 & 89 & 9.3 & 72 \\
\hline
\end{tabular}




\begin{tabular}{|c|c|c|c|c|c|c|c|}
\hline \multicolumn{3}{|c|}{$0.25 \mathrm{um} \mathrm{TiO}_{2} / \mathrm{Al}_{2} \mathrm{O}_{3}$} & \multirow[b]{2}{*}{$\begin{array}{c}\text { CONC } \\
\text { PRESSURE }\end{array}$} & \multirow[b]{2}{*}{$\begin{array}{c}\text { FEED FLOW } \\
\text { (L/min) }\end{array}$} & \multirow[b]{2}{*}{ FEED TEMP } & \multirow[b]{2}{*}{$\begin{array}{l}\text { PERMEATE } \\
\text { FLOW }\end{array}$} & \multirow[b]{2}{*}{$\begin{array}{l}\text { PERMEATE } \\
\text { FLUX }\end{array}$} \\
\hline $\begin{array}{c}\text { TIME OF } \\
\text { DAY }\end{array}$ & $\begin{array}{c}\text { ELAPSED } \\
\text { TIME }\end{array}$ & $\begin{array}{c}\text { FEED } \\
\text { PRESSURE }\end{array}$ & & & & & \\
\hline & (HH:MM) & (psig) & (psig) & (gpm) & $\left({ }^{\circ} \mathrm{F}\right)$ & (mU/min) & (gal/ftit-day) \\
\hline $15: 17$ & $0: 22$ & 29.0 & 7.0 & 0.8 & 90 & 9.3 & 72 \\
\hline $15: 17$ & $0: 23$ & 29.0 & 7.0 & 0.8 & 90 & 8.7 & 67 \\
\hline $15: 18$ & $0: 23$ & 29.0 & 7.0 & 0.8 & 89 & 8.7 & 67 \\
\hline $15: 18$ & $0: 24$ & 29.0 & 7.0 & 0.8 & 90 & 8.7 & 67 \\
\hline $15: 19$ & $0: 24$ & 28.0 & 7.0 & 0.8 & 89 & 8.7 & 67 \\
\hline $15: 19$ & $0: 25$ & 29.0 & 7.0 & 0.8 & 90 & 8.7 & 67 \\
\hline $15: 20$ & $0: 25$ & 28.0 & 7.0 & 0.8 & 89 & 8.7 & 67 \\
\hline $15: 21$ & $0: 26$ & 28.0 & 7.0 & 0.8 & 90 & 8.7 & 67 \\
\hline $15: 21$ & $0 ; 26$ & 30.0 & 7.0 & 0.8 & 90 & 8.7 & 67 \\
\hline $15: 22$ & $0: 27$ & 30.0 & 7.0 & 0.8 & 89 & 9.6 & 74 \\
\hline $15: 22$ & $0: 27$ & 30.0 & 7.0 & 0.8 & 90 & 9.6 & 74 \\
\hline $15: 23$ & $0: 28$ & 29.0 & 7.0 & 0.8 & 89 & 7.6 & 59 \\
\hline $15: 23$ & $0: 29$ & 29.0 & 7.0 & 0.8 & 89 & 7.6 & 59 \\
\hline $15: 24$ & $0: 29$ & 29.0 & 7.0 & 0.8 & 90 & 7.6 & 59 \\
\hline $15: 24$ & $0: 30$ & 29.0 & 7.0 & 0.8 & 89 & 7.6 & 59 \\
\hline $15: 25$ & $0: 30$ & 29.0 & 7.0 & 0.8 & 90 & 7.6 & 59 \\
\hline $15: 25$ & $0: 31$ & 29.0 & 7.0 & 0.8 & 89 & 7.6 & 59 \\
\hline $15: 26$ & $0: 31$ & 29.0 & 7.0 & 0.8 & 90 & 7.6 & 59 \\
\hline $15: 26$ & $0: 32$ & 29.0 & 7.0 & 0.8 & 90 & 7.6 & 59 \\
\hline $15: 27$ & $0: 32$ & 29.0 & 7.0 & 0.8 & $\overline{89}$ & 8.5 & 66 \\
\hline $15: 27$ & $0: 33$ & 29.0 & 7.0 & 0.8 & 90 & 8.5 & 66 \\
\hline $15: 28$ & $0: 33$ & 29.0 & 7.0 & 0.8 & 89 & 8.5 & 66 \\
\hline $15: 28$ & $0: 34$ & 29.0 & 7.0 & 0.8 & 90 & 8.5 & 66 \\
\hline $15: 29$ & $0: 34$ & 29.0 & 7.0 & 0.8 & 90 & 8.5 & 66 \\
\hline $15: 29$ & $0: 35$ & 29.0 & 7.0 & 0.8 & 89 & 8.4 & 65 \\
\hline $15: 30$ & $0: 35$ & 29.0 & 7.0 & 0.8 & 90 & 8.4 & 65 \\
\hline $15: 30$ & $0: 36$ & 29.0 & 7.0 & 0.8 & 90 & 8.4 & 65 \\
\hline $15: 31$ & $0: 36$ & 29.0 & 7.0 & 0.8 & 90 & 8.4 & 65 \\
\hline $15: 31$ & $0: 37$ & 29.0 & 7.0 & 0.8 & 90 & 8.4 & 65 \\
\hline $15: 32$ & $0: 37$ & 29.0 & 7.0 & 0.8 & 89 & 8.4 & 65 \\
\hline $15: 32$ & $.0: 38$ & 29.0 & 7.0 & 0.8 & 90 & 8.4 & 65 \\
\hline $15: 33$ & $0: 38$ & 29.0 & 7.0 & 0.8 & 89 & 8.4 & 65 \\
\hline $15: 33$ & $0: 39$ & 29.0 & 7.0 & 0.8 & 89 & 8.4 & 65 \\
\hline $15: 34$ & $0: 39$ & 29.0 & 7.0 & 0.8 & 90 & 8.4 & 65 \\
\hline $15: 34$ & $0: 40$ & 29.0 & 7.0 & 0.8 & 89 & 8.4 & 65 \\
\hline $15: 35$ & $0: 40$ & 29.0 & 7.0 & 0.8 & 90 & 8.4 & 65 \\
\hline $15: 35$ & $0: 41$ & 29.0 & 7.0 & 0.8 & 89 & 8.4 & 65 \\
\hline $15: 36$ & $0: 41$ & 28.0 & 7.0 & 0.8 & 89 & 7.6 & 59 \\
\hline $15: 36$ & $0: 42$ & 29.0 & 7.0 & 0.8 & 90 & 7.6 & 59 \\
\hline $15: 37$ & $0: 42$ & 29.0 & 7.0 & 0.8 & 89 & 7.6 & 59 \\
\hline $15: 37$ & $0: 43$ & 28.0 & 6.0 & 0.8 & 90 & 7.6 & 59 \\
\hline $15: 38$ & $0: 43$ & 28.0 & 6.0 & 0.8 & 89 & 7.6 & 59 \\
\hline $15: 39$ & $0: 44$ & 28.0 & 6.0 & 0.8 & 90 & 8.0 & 62 \\
\hline $15: 39$ & $0: 44$ & 28.0 & 6.0 & 0.8 & 90 & 8.0 & 62 \\
\hline $15: 40$ & $0: 45$ & 28.0 & 6.0 & 0.8 & 89 & 8.0 & 62 \\
\hline
\end{tabular}




\begin{tabular}{|c|c|c|c|c|c|c|c|}
\hline \multicolumn{3}{|c|}{0.25 um $\mathrm{TiO}_{2} / \mathrm{Al}_{2} \mathrm{O}_{3}$} & \multirow[b]{2}{*}{$\begin{array}{c}\text { CONC } \\
\text { PRESSURE }\end{array}$} & \multirow[b]{2}{*}{$\begin{array}{c}\text { FEED FLOW } \\
\text { (L/min) }\end{array}$} & \multirow[b]{2}{*}{ FEED TEMP } & \multirow[b]{2}{*}{$\begin{array}{l}\text { PERMEATE } \\
\text { FLOW }\end{array}$} & \multirow[b]{2}{*}{$\begin{array}{l}\text { PERMEATE } \\
\text { FLUX }\end{array}$} \\
\hline $\begin{array}{l}\text { TIME OF } \\
\text { DAY }\end{array}$ & $\begin{array}{c}\text { ELAPSED } \\
\text { TIME }\end{array}$ & $\begin{array}{c}\text { FEED } \\
\text { PRESSURE }\end{array}$ & & & & & \\
\hline & (HH:MM) & (psig) & (psig) & (gpm) & $\left({ }^{\circ} \mathrm{F}\right)$ & (ml/min) & (gal/ft ${ }^{2}$-day) \\
\hline $15: 40$ & $0: 46$ & 28.0 & 6.0 & 0.8 & 90 & 7.7 & 59 \\
\hline $15: 41$ & $0: 46$ & 28.0 & 6.0 & 0.8 & 89 & 7.7 & 59 \\
\hline $15: 41$ & $0: 47$ & 28.0 & 7.0 & 0.8 & 90 & 7.7 & 59 \\
\hline $15: 42$ & $0: 47$ & 28.0 & 6.0 & 0.8 & 90 & 7.7 & 59 \\
\hline $15: 42$ & $0: 48$ & 28.0 & 6.0 & 0.8 & 89 & 7.7 & 59 \\
\hline $15: 43$ & $0: 48$ & 27.0 & 6.0 & 0.8 & 90 & 7.7 & 59 \\
\hline $15: 43$ & $0: 49$ & 27.0 & 6.0 & 0.8 & 90 & 7.7 & 59 \\
\hline $15: 44$ & $0: 49$ & 27.0 & 6.0 & 0.8 & 89 & 7.7 & 59 \\
\hline $15: 44$ & $0: 50$ & 27.0 & 6.0 & 0.8 & 90 & 8.0 & 62 \\
\hline $15: 45$ & $0: 50$ & 27.0 & 6.0 & 0.8 & 89 & 8.0 & 62 \\
\hline $15: 45$ & $0: 51$ & 27.0 & 6.0 & 0.8 & 90 & 8.0 & 62 \\
\hline $15: 46$ & $0: 51$ & 27.0 & 6.0 & 0.8 & 90 & 8.0 & 62 \\
\hline $15: 46$ & $0: 52$ & 27.0 & 6.0 & 0.8 & 89 & 8.0 & 62 \\
\hline $15: 47$ & $0: 52$ & 27.0 & 6.0 & 0.8 & 90 & 8.0 & 62 \\
\hline $15: 47$ & $0: 53$ & 27.0 & 6.0 & 0.8 & 89 & 8.0 & 62 \\
\hline $15: 48$ & $0: 53$ & 27.0 & 6.0 & 0.8 & 90 & 8.0 & 62 \\
\hline $15: 48$ & $0: 54$ & 27.0 & 6.0 & 0.8 & 90 & 8.0 & 62 \\
\hline $15: 49$ & $0: 54$ & 27.0 & 6.0 & 0.8 & 89 & 8.0 & 62 \\
\hline $15: 49$ & $0: 55$ & 27.0 & 6.0 & 0.8 & 90 & 8.0 & 62 \\
\hline $15: 50$ & $0: 55$ & 27.0 & 6.0 & 0.8 & 89 & 8.0 & 62 \\
\hline $15: 50$ & $0: 56$ & 27.0 & 6.0 & 0.8 & 90 & 8.0 & 62 \\
\hline $15: 51$ & $0: 56$ & 27.0 & 6.0 & 0.8 & 90 & 8.0 & 62 \\
\hline $15: 51$ & $0: 57$ & 27.0 & 6.0 & 0.8 & 89 & 7.0 & 54 \\
\hline $15: 52$ & $0: 57$ & 27.0 & 6.0 & 0.8 & 90 & 7.0 & 54 \\
\hline $15: 52$ & $0: 58$ & 27.0 & 6.0 & 0.8 & 90 & 7.0 & 54 \\
\hline $15: 53$ & $0: 58$ & 27.0 & 6.0 & 0.8 & 89 & 7.0 & 54 \\
\hline $15: 54$ & $0: 59$ & 27.0 & 6.0 & 0.8 & 90 & 7.0 & 54 \\
\hline $15: 54$ & $0: 59$ & 27.0 & 6.0 & 0.8 & 89 & 7.0 & 54 \\
\hline $15: 55$ & $1: 00$ & 27.0 & 6.0 & 0.8 & 90 & 7.7 & 59 \\
\hline Average & ? & 28.4 & 6.6 & 0.8 & 89.6 & 8.3 & 63.8 \\
\hline & & & & \multirow{2}{*}{\multicolumn{3}{|c|}{ Feed Pressure $=28.4 \mathrm{nsia}$}} & \\
\hline \multicolumn{4}{|c|}{ Average Test Conditions: SCRN1.XLS } & & & & \\
\hline & & & & \multicolumn{3}{|c|}{ Concentrate Pressure $=6.6 \mathrm{psig}$} & \\
\hline \multirow{2}{*}{\multicolumn{2}{|c|}{ Date Run: 20 -Apr-96 }} & & & \multicolumn{3}{|c|}{ Feed Flow $=0.8 \mathrm{gpm}$} & \\
\hline & & & & \multicolumn{3}{|c|}{ Temp. $=90 \mathrm{~F}$} & \\
\hline
\end{tabular}


SCRN1.XLS

\begin{tabular}{|c|c|c|c|c|c|c|c|}
\hline SCRN1 & & & & & & & \\
\hline $0.25 \mathrm{ur}$ & $\mathrm{TiO}_{2} / \mathrm{S}$ & $O_{2}$ & & & & & \\
\hline $\begin{array}{c}\text { TIME OF } \\
\text { DAY }\end{array}$ & $\begin{array}{c}\text { ELAPSED } \\
\text { TIME }\end{array}$ & $\begin{array}{c}\text { FEED } \\
\text { PRESSURE }\end{array}$ & $\begin{array}{c}\text { CONC } \\
\text { PRESSURE }\end{array}$ & $\begin{array}{c}\text { FEED FLOW } \\
\text { (L/min) }\end{array}$ & FEED TEMP & $\begin{array}{l}\text { PERMEATE } \\
\text { FLOW }\end{array}$ & $\begin{array}{l}\text { PERMEATE } \\
\text { FLUX }\end{array}$ \\
\hline & (HH:MM) & (psig) & (psig) & (gpm) & $\left({ }^{\circ} \mathrm{F}\right)$ & $(\mathrm{m} / \mathrm{min})$ & (gal/ft ${ }^{2}$-day) \\
\hline $14: 54$ & $0: 00$ & 29.0 & 7.0 & 0.8 & 89 & 7.7 & 59 \\
\hline $14: 55$ & $0: 00$ & 28.0 & 7.0 & 0.8 & 89 & 7.7 & 59 \\
\hline $14: 55$ & $0: 01$ & 32.0 & 7.0 & 0.9 & 90 & 9.7 & 75 \\
\hline $14: 56$ & $0: 01$ & 31.0 & 7.0 & 0.9 & 89 & 9.7 & 75 \\
\hline $14: 56$ & $0: 02$ & 31.0 & 7.0 & 0.9 & 90 & 9.7 & 75 \\
\hline $14: 57$ & $0: 02$ & 31.0 & 7.0 & 0.9 & 90 & 9.7 & 75 \\
\hline $14: 57$ & $0: 03$ & 31.0 & 7.0 & 0.9 & 89 & 9.7 & 75 \\
\hline $14: 58$ & $0: 03$ & 31.0 & 7.0 & 0.9 & 90 & 9.7 & 75 \\
\hline $14: 58$ & $0: 04$ & 31.0 & 7.0 & 0.9 & 89 & 9.7 & 75 \\
\hline $14: 59$ & $0: 04$ & 30.0 & 7.0 & 0.9 & 90 & 9.9 & 76 \\
\hline $14: 59$ & $0: 05$ & 27.0 & 6.0 & 0.8 & 90 & 9.9 & 76 \\
\hline $15: 00$ & $0: 05$ & 27.0 & 6.0 & 0.8 & 89 & 9.9 & 76 \\
\hline $15: 00$ & $0: 06$ & 27.0 & 6.0 & 0.8 & 90 & 9.9 & 76 \\
\hline $15: 01$ & $0: 06$ & 30.0 & 7.0 & 0.8 & 89 & 9.9 & 76 \\
\hline $15: 01$ & $0: 07$ & 30.0 & 7.0 & 0.9 & 90 & 9.9 & 76 \\
\hline $15: 02$ & $0: 07$ & 30.0 & 7.0 & 0.8 & 90 & 9.9 & 76 \\
\hline $15: 02$ & $0: 08$ & 28.0 & 7.0 & 0.8 & 89 & 9.7 & 75 \\
\hline $15: 03$ & $0: 08$ & 28.0 & 7.0 & 0.8 & 90 & 9.7 & 75 \\
\hline $15: 03$ & $0: 09$ & 27.0 & 7.0 & 0.8 & 89 & 9.7 & 75 \\
\hline $15: 04$ & $0: 09$ & 27.0 & 6.0 & 0.8 & 89 & 9.7 & 75 \\
\hline $15: 04$ & $0: 10$ & 27.0 & 7.0 & $0 . \overline{0}$ & 90 & 9.7 & 75 \\
\hline $15: 05$ & $0: 10$ & 27.0 & 7.0 & 0.8 & 89 & 9.7 & 75 \\
\hline $15: 05$ & $0: 11$ & 27.0 & 6.0 & 0.8 & 90 & 9.7 & 75 \\
\hline $15: 06$ & $0: 11$ & 27.0 & 6.0 & 0.8 & 90 & 9.7 & 75 \\
\hline $15: 06$ & $0: 12$ & 27.0 & 6.0 & 0.8 & 90 & 8.9 & 69 \\
\hline $15: 07$ & $0: 12$ & 27.0 & 6.0 & 0.8 & 90 & 8.9 & 69 \\
\hline 15:08 & $0: 13$ & 27.0 & 6.0 & 0.8 & 89 & 8.9 & 69 \\
\hline $15: 08$ & $0: 13$ & 27.0 & 6.0 & 0.8 & 90 & 8.9 & 69 \\
\hline $15: 09$ & $0: 14$ & 27.0 & 6.0 & 0.8 & 89 & 8.9 & 69 \\
\hline $15: 09$ & $0: 14$ & 27.0 & 7.0 & 0.8 & 89 & 8.9 & 69 \\
\hline $15: 10$ & $0: 15$ & 30.0 & 7.0 & 0.8 & 90 & 8.9 & 69 \\
\hline $15: 10$ & $0: 15$ & 29.0 & 7.0 & 0.8 & 89 & 9.7 & 75 \\
\hline $15: 11$ & $0: 16$ & 29.0 & 7.0 & 0.8 & 90 & 9.7 & 75 \\
\hline $15: 11$ & $0: 16$ & 29.0 & 7.0 & 0.8 & 89 & 8.0 & 62 \\
\hline $15: 12$ & $0: 17$ & 29.0 & 7.0 & 0.8 & 89 & 8.0 & 62 \\
\hline $15: 12$ & $0: 18$ & 29.0 & 7.0 & 0.8 & 90 & 8.0 & 62 \\
\hline $15: 13$ & $0: 18$ & 29.0 & 7.0 & 0.8 & 89 & 8.0 & 62 \\
\hline $15: 13$ & $0: 19$ & 29.0 & 7.0 & 0.8 & 90 & 8.0 & 62 \\
\hline $15: 14$ & $0: 19$ & 29.0 & 7.0 & 0.8 & 89 & 8.0 & 62 \\
\hline $15: 14$ & $0: 20$ & 29.0 & 7.0 & 0.8 & 89 & 8.0 & 62 \\
\hline $15: 15$ & $0: 20$ & 29.0 & 7.0 & 0.8 & 90 & 8.0 & 62 \\
\hline $15: 15$ & $0: 21$ & 29.0 & 7.0 & 0.8 & 89 & 8.0 & 62 \\
\hline $15: 16$ & $0: 21$ & 29.0 & 7.0 & 0.8 & 90 & 8.0 & 62 \\
\hline $15: 16$ & $=0: 22$ & 29.0 & 7.0 & 0.8 & 89 & 8.0 & 62 \\
\hline
\end{tabular}


SCRN1.XLS

\begin{tabular}{|c|c|c|c|c|c|c|c|}
\hline \multicolumn{8}{|c|}{$0.25 \mathrm{um} \mathrm{TiO}_{2} / \mathrm{SiO}_{2}$} \\
\hline $\begin{array}{c}\text { TIME OF } \\
\text { DAY }\end{array}$ & $\begin{array}{l}\text { ELAPSED } \\
\text { TIME }\end{array}$ & $\begin{array}{c}\text { FEED } \\
\text { PRESSURE }\end{array}$ & $\begin{array}{c}\text { CONC } \\
\text { PRESSURE }\end{array}$ & $\begin{array}{c}\text { FEED FLOW } \\
\text { (L/min) }\end{array}$ & FEED TEMP & $\begin{array}{l}\text { PERMEATE } \\
\text { FLOW }\end{array}$ & $\begin{array}{l}\text { PERMEATE } \\
\text { FLUX }\end{array}$ \\
\hline & (HH:MM) & (psig) & (psig) & (gpm) & $\left({ }^{\circ} \mathrm{F}\right)$ & (mU/min) & (gal/fft ${ }^{2}$-day) \\
\hline $15: 17$ & $0: 22$ & 29.0 & 7.0 & 0.8 & 90 & 8.0 & 62 \\
\hline $15: 17$ & $0: 23$ & 29.0 & 7.0 & 0.8 & 90 & 8.0 & 62 \\
\hline $15: 18$ & $0: 23$ & 29.0 & 7.0 & 0.8 & 89 & 8.0 & 62 \\
\hline 15:18 & $0: 24$ & 29.0 & 7.0 & 0.8 & 90 & 8.0 & 62 \\
\hline $15: 19$ & $0: 24$ & 28.0 & 7.0 & 0.8 & 89 & 8.8 & 68 \\
\hline $15: 20$ & $0: 25$ & 29.0 & 7.0 & 0.8 & 90 & 8.8 & 68 \\
\hline $15: 20$ & $0: 25$ & 28.0 & 7.0 & 0.8 & 89 & 8.8 & 68 \\
\hline $15: 21$ & $0: 26$ & 28.0 & 7.0 & 0.8 & 90 & 8.8 & 68 \\
\hline $15: 21$ & $0: 26$ & 30.0 & 7.0 & 0.8 & 90 & 8.8 & 68 \\
\hline $15: 22$ & $0: 27$ & 30.0 & 7.0 & 0.8 & 89 & 8.8 & 68 \\
\hline $15: 22$ & $0: 27$ & 30.0 & 7.0 & 0.8 & 90 & 8.8 & 68 \\
\hline $15: 23$ & $0: 28$ & 29.0 & 7.0 & 0.8 & 89 & 8.8 & 68 \\
\hline $15: 23$ & $0: 29$ & 29.0 & 7.0 & 0.8 & 89 & 8.8 & 68 \\
\hline $15: 24$ & $0: 29$ & 29.0 & 7.0 & 0.8 & 90 & 9.7 & 75 \\
\hline $15: 24$ & $0: 30$ & 29.0 & 7.0 & 0.8 & 89 & 9.7 & 75 \\
\hline $15: 25$ & $0: 30$ & 29.0 & 7.0 & 0.8 & 90 & 9.7 & 75 \\
\hline $15: 25$ & $0: 31$ & 29.0 & 7.0 & 0.8 & 89 & 9.7 & 75 \\
\hline $15: 26$ & $0: 31$ & 29.0 & 7.0 & 0.8 & 90 & 9.7 & 75 \\
\hline $15: 26$ & $0: 32$ & 29.0 & 7.0 & 0.8 & 90 & 9.7 & 75 \\
\hline $15: 27$ & $0: 32$ & 29.0 & 7.0 & 0.8 & 89 & 9.7 & 75 \\
\hline $15: 27$ & $0: 33$ & 29.0 & 7.0 & 0.8 & 90 & 9.7 & 75 \\
\hline $15: 28$ & $0: 33$ & 29.0 & 7.0 & 0.8 & 89 & 9.7 & 75 \\
\hline $15: 28$ & $0: 34$ & 29.0 & 7.0 & 0.8 & 90 & 9.7 & 75 \\
\hline $15: 29$ & $0: 34$ & 29.0 & 7.0 & 0.8 & 90 & 9.7 & 75 \\
\hline $15: 29$ & $0: 35$ & 29.0 & 7.0 & 0.8 & 89 & 9.7 & 75 \\
\hline $15: 30$ & $0: 35$ & 29.0 & 7.0 & 0.8 & 90 & 9.7 & 75 \\
\hline $15: 30$ & $0: 36$ & 29.0 & 7.0 & 0.8 & 90 & 10.5 & 81 \\
\hline $15: 31$ & $0: 36$ & 29.0 & 7.0 & 0.8 & 90 & 10.5 & 81 \\
\hline $15: 31$ & $0: 37$ & 29.0 & 7.0 & 0.8 & 90 & 10.5 & 81 \\
\hline $15: 32$ & $0: 37$ & 29.0 & 7.0 & 0.8 & 89 & 10.5 & 81 \\
\hline $15: 32$ & $0: 38$ & 29.0 & 7.0 & 0.8 & 90 & 10.5 & 81 \\
\hline $15: 33$ & $0: 38$ & 29.0 & 7.0 & 0.8 & 89 & 10.5 & 81 \\
\hline $15: 33$ & $0: 39$ & 29.0 & 7.0 & 0.8 & 89 & 10.5 & 81 \\
\hline $15: 34$ & $0: 39$ & 29.0 & 7.0 & 0.8 & 90 & 10.5 & 81 \\
\hline $15: 34$ & $0: 40$ & 29.0 & 7.0 & 0.8 & 89 & 9.2 & 71 \\
\hline $15: 35$ & $0: 40$ & 29.0 & 7.0 & 0.8 & 90 & 9.2 & 71 \\
\hline $15: 35$ & $0: 41$ & 29.0 & 7.0 & 0.8 & 89 & 9.2 & 71 \\
\hline $15: 36$ & $0: 41$ & 28.0 & 7.0 & 0.8 & 89 & 9.2 & 71 \\
\hline $15: 37$ & $0: 42$ & 29.0 & 7.0 & 0.8 & 90 & 9.2 & 71 \\
\hline $15: 37$ & $0: 42$ & 29.0 & 7.0 & 0.8 & 89 & 9.2 & 71 \\
\hline $15: 38$ & $0: 43$ & 28.0 & 6.0 & 0.8 & 90 & 9.2 & 71 \\
\hline $15: 38$ & $0: 43$ & 28.0 & 6.0 & 0.8 & 89 & 9.2 & 71 \\
\hline $15: 39$ & $0: 44$ & 28.0 & 6.0 & 0.8 & 90 & 9.2 & 71 \\
\hline $15: 39$ & $0: 44$ & 28.0 & 6.0 & 0.8 & 90 & 9.2 & 71 \\
\hline $15: 40$ & $=0: 45$ & 28.0 & 6.0 & 0.8 & 89 & 9.2 & 71 \\
\hline
\end{tabular}




\begin{tabular}{|c|c|c|c|c|c|c|c|}
\hline \multicolumn{8}{|c|}{$0.25 \mathrm{um} \mathrm{TiO}_{2} / \mathrm{SiO}_{2}$} \\
\hline \multirow[t]{2}{*}{$\begin{array}{c}\text { TIME OF } \\
\text { DAY }\end{array}$} & $\begin{array}{c}\text { ELAPSED } \\
\text { TIME }\end{array}$ & $\begin{array}{c}\text { FEED } \\
\text { PRESSURE }\end{array}$ & $\begin{array}{c}\text { CONC } \\
\text { PRESSURE }\end{array}$ & $\begin{array}{c}\text { FEED FLOW } \\
\text { (Lmin) }\end{array}$ & FEED TEMP & $\begin{array}{l}\text { PERMEATE } \\
\text { FLOW }\end{array}$ & $\begin{array}{l}\text { PERMEATE } \\
\text { FLUX }\end{array}$ \\
\hline & (HH:MM) & (psig) & (psig) & (gpm) & $\left({ }^{\circ} \mathrm{F}\right)$ & $(\mathrm{ml} / \mathrm{min})$ & (gal/ftt ${ }^{2}$-day) \\
\hline $15: 40$ & $0: 46$ & 28.0 & 6.0 & 0.8 & 90 & 9.2 & 71 \\
\hline $15: 41$ & $0: 46$ & 28.0 & 6.0 & 0.8 & 89 & 9.2 & 71 \\
\hline $15: 41$ & $0: 47$ & 28.0 & 7.0 & 0.8 & 90 & 8.6 & 66 \\
\hline $15: 42$ & $0: 47$ & 28.0 & 6.0 & 0.8 & 90 & 8.6 & 66 \\
\hline $15: 42$ & $0: 48$ & 28.0 & 6.0 & 0.8 & 89 & 8.6 & 66 \\
\hline $15: 43$ & $0: 48$ & 27.0 & 6.0 & 0.8 & 90 & 8.6 & 66 \\
\hline $15: 43$ & $0: 49$ & 27.0 & 6.0 & 0.8 & 90 & 8.6 & 66 \\
\hline $15: 44$ & $0: 49$ & 27.0 & 6.0 & 0.8 & 89 & 8.6 & 66 \\
\hline $15: 44$ & $0: 50$ & 27.0 & 6.0 & 0.8 & 90 & 8.6 & 66 \\
\hline $15: 45$ & $0: 50$ & 27.0 & 6.0 & 0.8 & 89 & 8.6 & 66 \\
\hline $15: 45$ & $0: 51$ & 27.0 & 6.0 & 0.8 & 90 & 8.6 & 66 \\
\hline $15: 46$ & $0: 51$ & 27.0 & 6.0 & 0.8 & 90 & 8.6 & 66 \\
\hline $15: 46$ & $0: 52$ & 27.0 & 6.0 & 0.8 & 89 & 8.6 & 66 \\
\hline $15: 47$ & $0: 52$ & 27.0 & 6.0 & 0.8 & 90 & 7.5 & 58 \\
\hline $15: 47$ & $0: 53$ & 27.0 & 6.0 & 0.8 & 89 & 7.5 & 58 \\
\hline $15: 48$ & $0: 53$ & 27.0 & 6.0 & 0.8 & 90 & 7.5 & 58 \\
\hline $15: 48$ & $0: 54$ & 27.0 & 6.0 & 0.8 & 90 & 7.5 & 58 \\
\hline $15: 49$ & $0: 54$ & 27.0 & 6.0 & 0.8 & 89 & 7.5 & 58 \\
\hline $15: 49$ & $0: 55$ & 27.0 & 6.0 & 0.8 & 90 & 7.5 & 58 \\
\hline $15: 50$ & $0: 55$ & 27.0 & 6.0 & 0.8 & 89 & 7.5 & 58 \\
\hline $15: 50$ & $0: 56$ & 27.0 & 6.0 & 0.8 & 90 & 7.5 & 58 \\
\hline $15: 51$ & $0: 56$ & 27.0 & 6.0 & 0.8 & 90 & 7.5 & 58 \\
\hline $15: 51$ & $0: 57$ & 27.0 & 6.0 & 0.8 & 89 & 7.5 & 58 \\
\hline $15: 52$ & $0: 57$ & 27.0 & 6.0 & 0.8 & 90 & 7.5 & 58 \\
\hline $15: 52$ & $0: 58$ & 27.0 & 6.0 & 0.8 & 90 & 7.5 & 58 \\
\hline $15: 53$ & $0: 58$ & 27.0 & 6.0 & 0.8 & 89 & 7.5 & 58 \\
\hline $15: 54$ & $0: 59$ & 27.0 & 6.0 & 0.8 & 90 & 7.5 & 58 \\
\hline $15: 54$ & $0: 59$ & 27.0 & 6.0 & 0.8 & 89 & 7.5 & 58 \\
\hline $15: 55$ & $1: 00$ & 27.0 & 6.0 & 0.8 & 90 & 7.5 & 58 \\
\hline Average & & 28.4 & 6.6 & 0.8 & 89.6 & 8.9 & 69.0 \\
\hline \multicolumn{4}{|c|}{ Average Test Conditions: SCRN1.XLS } & \multicolumn{3}{|c|}{ Feed Pressure $=28.4 \mathrm{psig}$} & \\
\hline & & & & \multicolumn{3}{|c|}{ Concentrate Pressure $=6.6 \mathrm{psig}$} & \\
\hline \multirow{2}{*}{\multicolumn{2}{|c|}{ Date Run: 20-Apr-96 }} & & & \multicolumn{3}{|c|}{ Feed Flow $=0.8 \mathrm{gpm}$} & \\
\hline & & & & \multicolumn{3}{|c|}{ Temp. $=90 \mathrm{~F}$} & \\
\hline
\end{tabular}




\begin{tabular}{|c|c|c|c|c|c|c|c|}
\hline SCRN1 & & & & & & & \\
\hline $0.3 \mathrm{um}$ & $\mathrm{rO}_{2} / \mathrm{Ti}$ & & & & & & \\
\hline $\begin{array}{c}\text { TIME OF } \\
\text { DAY }\end{array}$ & $\begin{array}{c}\text { ELAPSED } \\
\text { TIME }\end{array}$ & $\begin{array}{c}\text { FEED } \\
\text { PRESSURE }\end{array}$ & $\begin{array}{c}\text { CONC } \\
\text { PRESSURE }\end{array}$ & $\begin{array}{c}\text { FEED FLOW } \\
\text { (L/min) }\end{array}$ & FEED TEMP & $\begin{array}{c}\text { PERMEATE } \\
\text { FLOW }\end{array}$ & $\begin{array}{l}\text { PERMEATE } \\
\text { FLUX }\end{array}$ \\
\hline & (HH:MM) & (psig) & (psig) & (gpm) & $\left({ }^{\circ} \mathrm{F}\right)$ & (mL/min) & (gal/fti2-day) \\
\hline $14: 54$ & $0: 00$ & 29.0 & 7.0 & 0.8 & 89 & 9.8 & 76 \\
\hline $14: 55$ & $0: 00$ & 28.0 & 7.0 & 0.8 & 89 & 9.8 & 76 \\
\hline $14: 55$ & $0: 01$ & 32.0 & 7.0 & 0.9 & 90 & 9.8 & 76 \\
\hline $14: 56$ & $0: 01$ & 31.0 & 7.0 & 0.9 & 89 & 9.8 & 76 \\
\hline $14: 56$ & $0: 02$ & 31.0 & 7.0 & 0.9 & 90 & 9.8 & 76 \\
\hline $14: 57$ & $0: 02$ & 31.0 & 7.0 & 0.9 & 90 & 9.6 & 74 \\
\hline $14: 57$ & $0: 03$ & 31.0 & 7.0 & 0.9 & 89 & 9.6 & 74 \\
\hline $14: 58$ & $0: 03$ & 31.0 & 7.0 & 0.9 & 90 & 9.6 & 74 \\
\hline $14: 58$ & $0: 04$ & 31.0 & 7.0 & 0.9 & 89 & 9.6 & 74 \\
\hline $14: 59$ & $0: 04$ & 30.0 & 7.0 & 0.9 & 90 & 9.6 & 74 \\
\hline $14: 59$ & $0: 05$ & 27.0 & 6.0 & 0.8 & 90 & 9.6 & 74 \\
\hline $15: 00$ & $0: 05$ & 27.0 & 6.0 & 0.8 & 89 & 8.4 & 65 \\
\hline $15: 00$ & $0: 06$ & 27.0 & 6.0 & 0.8 & 90 & 8.4 & 65 \\
\hline $15: 01$ & $0: 06$ & 30.0 & 7.0 & 0.8 & 89 & 8.4 & 65 \\
\hline $15: 01$ & $0: 07$ & 30.0 & 7.0 & 0.9 & 90 & 8.4 & 65 \\
\hline $15: 02$ & $0: 07$ & 30.0 & 7.0 & 0.8 & 90 & 8.4 & 65 \\
\hline $15: 02$ & $0: 08$ & 28.0 & 7.0 & 0.8 & 89 & 8.4 & 65 \\
\hline $15: 03$ & $0: 08$ & 28.0 & 7.0 & 0.8 & 90 & 8.4 & 65 \\
\hline 15:03 & $0: 09$ & 27.0 & 7.0 & 0.8 & 89 & 8.4 & 65 \\
\hline $15: 04$ & $0: 09$ & 27.0 & 6.0 & 0.8 & 89 & 8.6 & 66 \\
\hline $15: 04$ & $0: 10$ & 27.0 & 7.0 & 0.8 & 90 & 8.6 & 66 \\
\hline $15: 05$ & $0: 10$ & 27.0 & 7.0 & 0.8 & 89 & 8.6 & 66 \\
\hline $15: 05$ & $0: 11$ & 27.0 & 6.0 & 0.8 & 90 & 8.6 & 66 \\
\hline $15: 06$ & $0: 11$ & 27.0 & 6.0 & 0.8 & 90 & 8.6 & 66 \\
\hline $15: 06$ & $0: 12$ & 27.0 & 6.0 & 0.8 & 90 & 8.6 & 66 \\
\hline $15: 07$ & $0: 12$ & 27.0 & 6.0 & 0.8 & 90 & 8.6 & 66 \\
\hline $15: 08$ & $0: 13$ & 27.0 & 6.0 & 0.8 & 89 & 8.5 & 66 \\
\hline $15: 08$ & $0: 13$ & 27.0 & 6.0 & 0.8 & 90 & 8.5 & 66 \\
\hline $15: 09$ & $0: 14$ & 27.0 & 6.0 & 0.8 & 89 & 8.5 & 66 \\
\hline $15: 09$ & $0: 14$ & 27.0 & 7.0 & 0.8 & 89 & 8.5 & 66 \\
\hline $15: 10$ & $0: 15$ & 30.0 & 7.0 & 0.8 & 90 & 8.5 & 66 \\
\hline $15: 10$ & $0: 15$ & 29.0 & 7.0 & 0.8 & 89 & 8.5 & 66 \\
\hline $15: 11$ & $0: 16$ & 29.0 & 7.0 & 0.8 & 90 & 8.5 & 66 \\
\hline $15: 11$ & $0: 16$ & 29.0 & 7.0 & 0.8 & 89 & 8.5 & 66 \\
\hline $15: 12$ & $0: 17$ & 29.0 & 7.0 & 0.8 & 89 & 8.5 & 66 \\
\hline $15: 12$ & $0: 18$ & 29.0 & 7.0 & 0.8 & 90 & 8.5 & 66 \\
\hline $15: 13$ & $0: 18$ & 29.0 & 7.0 & 0.8 & 89 & 8.5 & 66 \\
\hline $15: 13$ & $0: 19$ & 29.0 & 7.0 & 0.8 & 90 & 8.5 & 66 \\
\hline $15: 14$ & $0: 19$ & 29.0 & 7.0 & 0.8 & 89 & 8.8 & 68 \\
\hline $15: 14$ & $0: 20$ & 29.0 & 7.0 & 0.8 & 89 & 8.8 & 68 \\
\hline $15: 15$ & $0: 20$ & 29.0 & 7.0 & 0.8 & 90 & 8.8 & 68 \\
\hline $15: 15$ & $0: 21$ & 29.0 & 7.0 & 0.8 & 89 & 8.8 & 68 \\
\hline $15: 16$ & $0: 21$ & 29.0 & 7.0 & 0.8 & 90 & 8.8 & 68 \\
\hline $15: 16$ & $=0: 22$ & 29.0 & 7.0 & 0.8 & 89 & 7.6 & 59 \\
\hline
\end{tabular}




\begin{tabular}{|c|c|c|c|c|c|c|c|}
\hline \multicolumn{3}{|c|}{0.3 um $\mathrm{ZrO}_{2} / \mathrm{TiO}_{2}$} & \multirow[b]{2}{*}{$\begin{array}{c}\text { CONC } \\
\text { PRESSURE }\end{array}$} & \multirow[b]{2}{*}{$\begin{array}{l}\text { FEED FLOW } \\
\text { (Umin) }\end{array}$} & \multirow[b]{2}{*}{ FEED TEMP } & \multirow[b]{2}{*}{$\begin{array}{l}\text { PERMEATE } \\
\text { FLOW }\end{array}$} & \multirow[b]{2}{*}{$\begin{array}{l}\text { PERMEATE } \\
\text { FLUX }\end{array}$} \\
\hline$\underset{\text { DAY }}{\text { TIME OF }}$ & $\begin{array}{c}\text { ELAPSED } \\
\text { TIME }\end{array}$ & $\begin{array}{c}\text { FEED } \\
\text { PRESSURE }\end{array}$ & & & & & \\
\hline & (HH:MM) & (psig) & (psig) & (gpm) & $\left({ }^{\circ} \mathrm{F}\right)$ & $(\mathrm{ml} / \mathrm{min})$ & (gal/ft' -day) \\
\hline $15: 17$ & $0: 22$ & 29.0 & 7.0 & 0.8 & 90 & 7.6 & 59 \\
\hline $15: 17$ & $0: 23$ & 29.0 & 7.0 & 0.8 & 90 & 7.6 & 59 \\
\hline $15: 18$ & $0: 23$ & 29.0 & 7.0 & 0.8 & 89 & 7.6 & 59 \\
\hline $15: 18$ & $0: 24$ & 29.0 & 7.0 & 0.8 & 90 & 7.6 & 59 \\
\hline $15: 19$ & $0: 24$ & 28.0 & 7.0 & 0.8 & 89 & 7.6 & 59 \\
\hline $15: 20$ & $0: 25$ & 29.0 & 7.0 & 0.8 & 90 & 7.6 & 59 \\
\hline $15: 20$ & $0: 25$ & 28.0 & 7.0 & 0.8 & 89 & 9.7 & 75 \\
\hline $15: 21$ & $0: 26$ & 28.0 & 7.0 & 0.8 & 90 & 9.7 & 75 \\
\hline $15: 21$ & $0: 26$ & 30.0 & 7.0 & 0.8 & 90 & 9.7 & 75 \\
\hline $15: 22$ & $0: 27$ & 30.0 & 7.0 & 0.8 & 89 & 9.7 & 75 \\
\hline $15: 22$ & $0: 27$ & 30.0 & 7.0 & 0.8 & 90 & 9.7 & 75 \\
\hline $15: 23$ & $0: 28$ & 29.0 & 7.0 & 0.8 & 89 & 9.7 & 75 \\
\hline $15: 23$ & $0: 29$ & 29.0 & 7.0 & 0.8 & 89 & 9.7 & 75 \\
\hline $15: 24$ & $0: 29$ & 29.0 & 7.0 & 0.8 & 90 & 9.7 & 75 \\
\hline $15: 24$ & $0: 30$ & 29.0 & 7.0 & 0.8 & 89 & 9.7 & 75 \\
\hline $15: 25$ & $0: 30$ & 29.0 & 7.0 & 0.8 & 90 & 9.7 & 75 \\
\hline $15: 25$ & $0: 31$ & 29.0 & 7.0 & 0.8 & 89 & 8.3 & 64 \\
\hline $15: 26$ & $0: 31$ & 29.0 & 7.0 & 0.8 & 90 & 8.3 & 64 \\
\hline $15: 26$ & $0: 32$ & 29.0 & 7.0 & 0.8 & 90 & 8.3 & 64 \\
\hline $15: 27$ & $0: 32$ & 29.0 & 7.0 & 0.8 & 89 & 8.3 & 64 \\
\hline $15: 27$ & $0: 33$ & 29.0 & 7.0 & 0.8 & 90 & 8.3 & 64 \\
\hline $15: 28$ & $0: 33$ & 29.0 & 7.0 & 0.8 & 89 & 8.4 & 65 \\
\hline $15: 28$ & $0: 34$ & 29.0 & 7.0 & 0.8 & 90 & 8.4 & 65 \\
\hline $15: 29$ & $0: 34$ & 29.0 & 7.0 & 0.8 & 90 & 8.4 & 65 \\
\hline $15: 29$ & $0: 35$ & 29.0 & 7.0 & 0.8 & 89 & 8.4 & 65 \\
\hline $15: 30$ & $0: 35$ & 29.0 & 7.0 & 0.8 & 90 & 8.4 & 65 \\
\hline $15: 30$ & $0: 36$ & 29.0 & 7.0 & 0.8 & 90 & 8.4 & 65 \\
\hline $15: 31$ & $0: 36$ & 29.0 & 7.0 & 0.8 & 90 & 8.4 & 65 \\
\hline $15: 31$ & $0: 37$ & 29.0 & 7.0 & 0.8 & 90 & 8.4 & 65 \\
\hline $15: 32$ & $0: 37$ & 29.0 & 7.0 & 0.8 & 89 & 7.3 & 56 \\
\hline $15: 32$ & $0: 38$ & 29.0 & 7.0 & 0.8 & 90 & 7.3 & 56 \\
\hline $15: 33$ & $0: 38$ & 29.0 & 7.0 & 0.8 & 89 & 7.3 & 56 \\
\hline $15: 33$ & $0: 39$ & 29.0 & 7.0 & 0.8 & 89 & 7.3 & 56 \\
\hline $15: 34$ & $0: 39$ & 29.0 & 7.0 & 0.8 & 90 & 7.3 & 56 \\
\hline $15: 34$ & $0: 40$ & 29.0 & 7.0 & 0.8 & 89 & 7.3 & 56 \\
\hline $15: 35$ & $0: 40$ & 29.0 & 7.0 & 0.8 & 90 & 7.3 & 56 \\
\hline $15: 36$ & $0: 41$ & 29.0 & 7.0 & 0.8 & 89 & 7.3 & 56 \\
\hline $15: 36$ & $0: 41$ & 28.0 & 7.0 & 0.8 & 89 & 7.3 & 56 \\
\hline $15: 37$ & $0: 42$ & 29.0 & 7.0 & 0.8 & 90 & 7.3 & 56 \\
\hline $15: 37$ & $0: 42$ & 29.0 & 7.0 & 0.8 & 89 & 7.1 & 55 \\
\hline $15: 38$ & $0: 43$ & 28.0 & 6.0 & 0.8 & 90 & 7.1 & 55 \\
\hline $15: 38$ & $0: 43$ & 28.0 & 6.0 & 0.8 & 89 & 7.1 & 55 \\
\hline $15: 39$ & $0: 44$ & 28.0 & 6.0 & 0.8 & 90 & 7.1 & 55 \\
\hline $15: 39$ & $0: 44$ & 28.0 & 6.0 & 0.8 & 90 & 7.1 & 55 \\
\hline $15: 40$ & $=0: 45$ & 28.0 & 6.0 & 0.8 & 89 & 7.1 & 55 \\
\hline
\end{tabular}




\begin{tabular}{|c|c|c|c|c|c|c|c|}
\hline \multicolumn{3}{|c|}{0.3 um $\mathrm{ZrO}_{2} / \mathrm{TiO}_{2}$} & \multirow[b]{2}{*}{$\begin{array}{c}\text { CONC } \\
\text { PRESSURE }\end{array}$} & \multirow[b]{2}{*}{$\begin{array}{c}\text { FEED FLOW } \\
\text { (L/min) }\end{array}$} & \multirow[b]{2}{*}{ FEED TEMP } & \multirow[b]{2}{*}{$\begin{array}{c}\text { PERMEATE } \\
\text { FLOW }\end{array}$} & \multirow[b]{2}{*}{$\begin{array}{l}\text { PERMEATE } \\
\text { FLUX }\end{array}$} \\
\hline $\begin{array}{l}\text { TIME OF } \\
\text { DAY }\end{array}$ & $\begin{array}{c}\text { ELAPSED } \\
\text { TIME }\end{array}$ & $\begin{array}{c}\text { FEED } \\
\text { PRESSURE }\end{array}$ & & & & & \\
\hline & (HH:MM) & (psig) & (psig) & (gpm) & $\left({ }^{\circ} \mathrm{F}\right)$ & $(\mathrm{ml} / \mathrm{min})$ & (gal/ft²-day) \\
\hline $15: 40$ & $0: 46$ & 28.0 & 6.0 & 0.8 & 90 & 7.1 & 55 \\
\hline $15: 41$ & $0: 46$ & 28.0 & 6.0 & 0.8 & 89 & 7.1 & 55 \\
\hline $15: 41$ & $0: 47$ & 28.0 & 7.0 & 0.8 & 90 & 7.1 & 55 \\
\hline $15: 42$ & $0: 47$ & 28.0 & 6.0 & 0.8 & 90 & 7.1 & 55 \\
\hline $15: 42$ & $0: 48$ & 28.0 & 6.0 & 0.8 & 89 & 7.1 & $55^{\circ}$ \\
\hline $15: 43$ & $0: 48$ & 27.0 & 6.0 & 0.8 & 90 & 8.0 & 62 \\
\hline $15: 43$ & $0: 49$ & 27.0 & 6.0 & 0.8 & 90 & 8.0 & 62 \\
\hline $15: 44$ & $0: 49$ & 27.0 & 6.0 & 0.8 & 89 & 8.0 & 62 \\
\hline $15: 44$ & $0: 50$ & 27.0 & 6.0 & 0.8 & 90 & 8.0 & 62 \\
\hline $15: 45$ & $0: 50$ & 27.0 & 6.0 & 0.8 & 89 & 8.0 & 62 \\
\hline $15: 45$ & $0: 51$ & 27.0 & 6.0 & 0.8 & 90 & 8.0 & 62 \\
\hline $15: 46$ & $0: 51$ & 27.0 & 6.0 & 0.8 & 90 & 6.6 & 51 \\
\hline $15: 46$ & $0: 52$ & 27.0 & 6.0 & 0.8 & 89 & 6.6 & 51 \\
\hline $15: 47$ & $0: 52$ & 27.0 & 6.0 & 0.8 & 90 & 6.6 & 51 \\
\hline $15: 47$ & $0: 53$ & 27.0 & 6.0 & 0.8 & 89 & 6.6 & 51 \\
\hline $15: 48$ & $0: 53$ & 27.0 & 6.0 & 0.8 & 90 & 6.6 & 51 \\
\hline $15: 48$ & $0: 54$ & 27.0 & 6.0 & 0.8 & 90 & 7.2 & 56 \\
\hline $15: 49$ & $0: 54$ & 27.0 & 6.0 & 0.8 & 89 & 7.2 & 56 \\
\hline $15: 49$ & $0: 55$ & 27.0 & 6.0 & 0.8 & 90 & 7.2 & 56 \\
\hline $15: 50$ & $0: 55$ & 27.0 & 6.0 & 0.8 & 89 & 7.3 & 56 \\
\hline $15: 50$ & $0: 56$ & 27.0 & 6.0 & 0.8 & 90 & 7.3 & 56 \\
\hline $15: 51$ & $0: 56$ & 27.0 & 6.0 & 0.8 & 90 & 7.3 & 56 \\
\hline $15: 51$ & $0: 57$ & 27.0 & 6.0 & 0.8 & 89 & 7.3 & 56 \\
\hline $15: 52$ & $0: 57$ & 27.0 & 6.0 & 0.8 & 90 & 7.3 & 56 \\
\hline $15: 52$ & $0: 58$ & 27.0 & 6.0 & 0.8 & 90 & 7.3 & 56 \\
\hline $15: 53$ & $0: 58$ & 27.0 & 6.0 & 0.8 & 89 & 7.1 & 55 \\
\hline $15: 54$ & $0: 59$ & 27.0 & 6.0 & 0.8 & 90 & 7.1 & 55 \\
\hline $15: 54$ & $0: 59$ & 27.0 & 6.0 & 0.8 & 89 & 7.1 & 55 \\
\hline $15: 55$ & $1: 00$ & 27.0 & 6.0 & 0.8 & 90 & 7.1 & 55 \\
\hline Average & & 28.4 & 6.6 & 0.8 & 89.6 & 8.2 & 63.1 \\
\hline & & & & & & & \\
\hline \multicolumn{4}{|c|}{ Average Test Conditions: SCRN1.XLS } & \multicolumn{3}{|c|}{ Feed Pressure $=28.4$ psig } & \\
\hline & & & & \multicolumn{3}{|c|}{ Concentrate Pressure $=6.6$ psig } & \\
\hline \multirow{2}{*}{\multicolumn{2}{|c|}{ Date Run: 20-Apr-96 }} & & & \multicolumn{3}{|c|}{ Feed Flow $=0.8 \mathrm{gpm}$} & \\
\hline & & & & \multicolumn{3}{|c|}{ Temp. $=90 \mathrm{~F}$} & \\
\hline
\end{tabular}




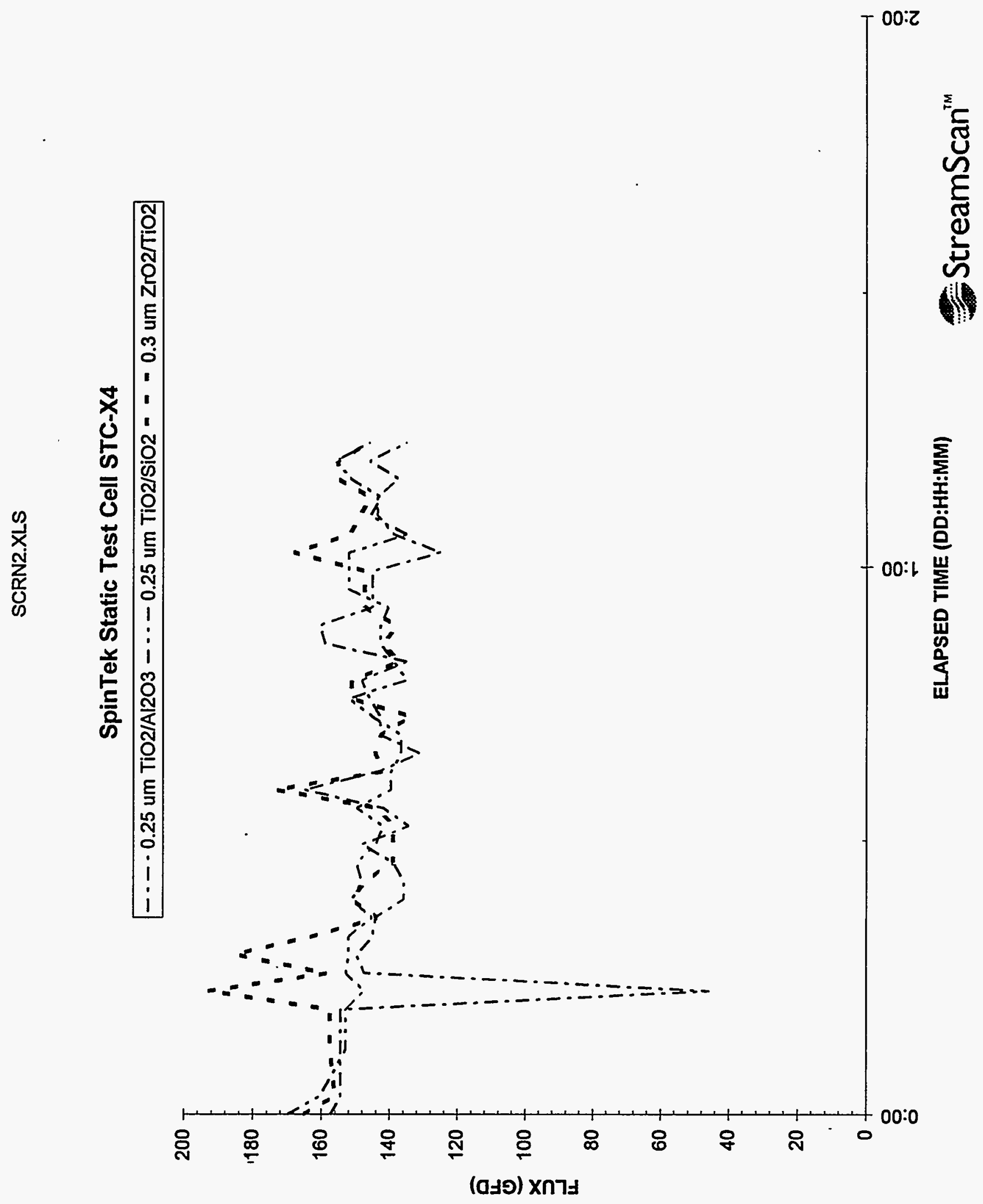




\begin{tabular}{|c|c|c|c|c|c|c|c|}
\hline \multicolumn{5}{|l|}{ SCRN2 } & & & \\
\hline \multicolumn{8}{|c|}{0.25 um $\mathrm{TiO}_{2} / \mathrm{Al}_{2} \mathrm{O}_{3}$} \\
\hline \multirow[t]{2}{*}{$\begin{array}{l}\text { TIME OF } \\
\text { DAY }\end{array}$} & $\begin{array}{l}\text { ELAPSED } \\
\text { TIME }\end{array}$ & $\begin{array}{c}\text { FEED } \\
\text { PRESSURE }\end{array}$ & $\begin{array}{c}\text { CONC } \\
\text { PRESSURE }\end{array}$ & $\begin{array}{c}\text { FEED FLOW } \\
\text { (L'min) }\end{array}$ & FEED TEMP & $\begin{array}{l}\text { PERMEATE } \\
\text { FLOW }\end{array}$ & $\begin{array}{l}\text { PERMEATE } \\
\text { FLUX }\end{array}$ \\
\hline & (HH:MM) & (psig) & (psig) & (gpm) & $\left({ }^{\circ} \mathrm{F}\right)$ & $(\mathrm{m} / \mathrm{min})$ & (gal/fiteday) \\
\hline $19: 16$ & $0: 00$ & 75.0 & 42.0 & 1.0 & 90 & 20.4 & 157 \\
\hline $19: 18$ & $0: 01$ & 75.0 & 42.0 & 1.0 & 89 & 20.0 & 154 \\
\hline $19: 23$ & $0: 07$ & 75.0 & 42.0 & 1.0 & 89 & 20.0 & 154 \\
\hline $19: 24$ & $0: 08$ & 75.0 & 42.0 & 1.0 & 89 & 20.0 & 154 \\
\hline $19: 27$ & $0: 11$ & 75.0 & 42.0 & 1.0 & 89 & 20.0 & 154 \\
\hline $19: 29$ & $0: 13$ & 75.0 & 42.0 & 1.0 & 89 & 5.9 & 46 \\
\hline $19: 31$ & $0: 15$ & 75.0 & 42.0 & 1.0 & 89 & 19.1 & 147 \\
\hline $19: 33$ & $0: 17$ & 75.0 & 42.0 & 1.0 & 90 & 19.4 & 150 \\
\hline $19: 35$ & $0: 19$ & 75.0 & 41.0 & 1.0 & 90 & 18.8 & 145 \\
\hline $19: 37$ & $0: 21$ & 75.0 & 42.0 & 1.0 & 90 & 18.7 & 144 \\
\hline $19: 39$ & $0: 23$ & 75.0 & 42.0 & 1.0 & 90 & 17.6 & 136 \\
\hline $19: 41$ & $0: 25$ & 75.0 & 41.0 & 1.0 & 90 & 17.6 & 136 \\
\hline $19: 43$ & $0: 27$ & 75.0 & 41.0 & 1.0 & 89 & 18.0 & 139 \\
\hline $19: 45$ & $0: 29$ & 75.0 & 42.0 & 1.0 & 89 & 19.2 & 148 \\
\hline $19: 47$ & $0: 31$ & 75.0 & 41.0 & 1.0 & 90 & 17.4 & 134 \\
\hline $19: 49$ & $0: 33$ & 75.0 & 41.0 & 1.0 & 89 & 18.4 & 142 \\
\hline $19: 51$ & $0: 35$ & 75.0 & 41.0 & 1.0 & 90 & 21.4 & 165 \\
\hline $19: 53$ & $0: 37$ & 75.0 & 41.0 & 1.0 & 90 & 18.5 & 143 \\
\hline $19: 55$ & $0: 39$ & 75.0 & 41.0 & 1.0 & 90 & 17.0 & 131 \\
\hline $19: 57$ & $0: 41$ & 75.0 & 41.0 & 1.0 & 90 & 18.5 & 143 \\
\hline $19: 59$ & $0: 43$ & 75.0 & 41.0 & 1.0 & 90 & 18.5 & 143 \\
\hline $20: 01$ & $0: 45$ & 75.0 & 41.0 & 1.0 & 89 & 18.9 & 146 \\
\hline $20: 03$ & $0: 47$ & 75.0 & 41.0 & 1.0 & 90 & 19.2 & 148 \\
\hline $20: 05$ & $0: 49$ & 75.0 & 41.0 & 1.0 & 90 & 17.5 & 135 \\
\hline $20: 07$ & $0: 51$ & 75.0 & 41.0 & 1.0 & 89 & 20.6 & 159 \\
\hline $20: 09$ & $0: 53$ & 75.0 & 42.0 & 1.0 & 90 & 20.8 & 161 \\
\hline $20: 11$ & $0: 55$ & 75.0 & 42.0 & 1.0 & 90 & 18.8 & 145 \\
\hline $20: 13$ & $0: 57$ & 0.0 & 0.0 & 0.0 & 89 & 18.8 & 145 \\
\hline $20: 15$ & $0: 59$ & 74.0 & 41.0 & 0.7 & 89 & 18.8 & 145 \\
\hline $20: 17$ & $1: 01$ & 75.0 & 41.0 & 1.0 & 89 & 16.2 & 125 \\
\hline $20: 19$ & $1: 03$ & 75.0 & 42.0 & 1.0 & 90 & 18.0 & 139 \\
\hline $20: 21$ & $1: 05$ & 75.0 & 42.0 & 1.0 & 90 & 18.6 & 144 \\
\hline $20: 23$ & $1: 07$ & 75.0 & 41.0 & 1.0 & 90 & 18.6 & 144 \\
\hline $20: 25$ & $1: 09$ & 75.0 & 41.0 & 1.0 & 90 & 17.8 & 137 \\
\hline $20: 27$ & $1: 11$ & 75.0 & 41.0 & 1.0 & 90 & 18.9 & 146 \\
\hline $20: 30$ & $1: 13$ & 75.0 & 41.0 & 1.0 & 90 & 17.5 & 135 \\
\hline Average & & 75.0 & 41.4 & 1.0 & 89.6 & 18.4 & 142.1 \\
\hline \multicolumn{4}{|c|}{$\begin{array}{l}1 \\
\text { Average Test Conditions: SCRN2.XLS } \\
\end{array}$} & \multicolumn{4}{|c|}{$\frac{1}{\text { Feed Pressure }=72.5 \mathrm{psig}}$} \\
\hline & & & & \multicolumn{4}{|c|}{ Concentrate Pressure $=39.9$ psig } \\
\hline \multicolumn{2}{|c|}{ Date Run: 20-Apr-96 } & & & \multicolumn{3}{|c|}{ Feed Flow $=1.0 \mathrm{gpm}$} & \\
\hline & & & & & Temp. & $=90 \mathrm{~F}$ & \\
\hline
\end{tabular}




\begin{tabular}{|c|c|c|c|c|c|c|c|}
\hline \multicolumn{8}{|l|}{ SCRN2 } \\
\hline \multicolumn{8}{|c|}{0.25 um $\mathrm{TiO}_{2} / \mathrm{SiO}_{2}$} \\
\hline \multirow[t]{2}{*}{$\begin{array}{c}\text { TIME OF } \\
\text { DAY }\end{array}$} & $\begin{array}{c}\text { ELAPSED } \\
\text { TIME }\end{array}$ & $\begin{array}{c}\text { FEED } \\
\text { PRESSURE }\end{array}$ & $\begin{array}{c}\text { CONC } \\
\text { PRESSURE }\end{array}$ & $\begin{array}{c}\text { FEED FLOW } \\
\text { (Lmin) }\end{array}$ & FEED TEMP & $\begin{array}{l}\text { PERMEATE } \\
\text { FLOW }\end{array}$ & $\begin{array}{l}\text { PERMEATE } \\
\text { FLUX }\end{array}$ \\
\hline & (HH:MM) & (psig) & (psig) & (gpm) & $\left({ }^{\circ} \mathrm{F}\right)$ & $(\mathrm{m} / \mathrm{min})$ & (gal/fit ${ }^{2}$ day) \\
\hline $19: 16$ & $0: 00$ & 75.0 & 42.0 & 1.0 & 90 & 22.0 & 170 \\
\hline $19: 18$ & $0: 01$ & 75.0 & 42.0 & 1.0 & 89 & 20.8 & 161 \\
\hline $19: 23$ & $0: 07$ & 75.0 & 42.0 & 1.0 & 89 & 19.8 & 153 \\
\hline $19: 24$ & $0: 08$ & 75.0 & 42.0 & 1.0 & 89 & 19.8 & 153 \\
\hline $19: 27$ & $0: 11$ & 75.0 & 42.0 & 1.0 & 89 & 19.8 & 153 \\
\hline $19: 29$ & $0: 13$ & 75.0 & 42.0 & 1.0 & 89 & 19.1 & 147 \\
\hline $19: 31$ & $0: 15$ & 75.0 & 42.0 & 1.0 & 89 & 19.8 & 153 \\
\hline $19: 33$ & $0: 17$ & 75.0 & 42.0 & 1.0 & 90 & 19.7 & 152 \\
\hline $19: 35$ & $0: 19$ & 75.0 & 41.0 & 1.0 & 90 & 19.7 & 152 \\
\hline $19: 37$ & $0: 21$ & 75.0 & 42.0 & 1.0 & 90 & 18.8 & 145 \\
\hline $19: 39$ & $0: 23$ & 75.0 & 42.0 & 1.0 & 90 & 19.6 & 151 \\
\hline $19: 41$ & $0: 25$ & 75.0 & 41.0 & 1.0 & 90 & 19.2 & 148 \\
\hline $19: 43$ & $0: 27$ & 75.0 & 41.0 & 1.0 & 89 & 19.4 & 150 \\
\hline $19: 45$ & $0: 29$ & 75.0 & 42.0 & 1.0 & 89 & 18.7 & 144 \\
\hline $19: 47$ & $0: 31$ & 75.0 & 41.0 & 1.0 & 90 & 18.4 & 142 \\
\hline $19: 49$ & $0: 33$ & $75: 0$ & 41.0 & 1.0 & 89 & 19.4 & 150 \\
\hline $19: 51$ & $0: 35$ & 75.0 & 41.0 & 1.0 & 90 & 18.1 & 140 \\
\hline $19: 53$ & $0: 37$ & 75.0 & 41.0 & 1.0 & 90 & 18.1 & 140 \\
\hline $19: 55$ & $0: 39$ & 75.0 & 41.0 & 1.0 & 90 & 17.7 & 137 \\
\hline $19: 57$ & $0: 41$ & 75.0 & 41.0 & 1.0 & 90 & 17.7 & 137 \\
\hline $19: 59$ & $0: 43$ & 75.0 & 41.0 & 1.0 & 90 & 18.8 & 145 \\
\hline $20: 01$ & $0: 45$ & 75.0 & 41.0 & 1.0 & 89 & 19.7 & 152 \\
\hline $20: 03$ & $0: 47$ & 75.0 & 41.0 & 1.0 & 90 & 17.5 & 135 \\
\hline $20: 05$ & $0: 49$ & 75.0 & 41.0 & 1.0 & 90 & 18.0 & 139 \\
\hline $20: 07$ & $0: 51$ & 75.0 & 41.0 & 1.0 & 89 & 18.5 & 143 \\
\hline $20: 09$ & $0: 53$ & 75.0 & 42.0 & 1.0 & 90 & 18.5 & 143 \\
\hline $20: 11$ & $0: 55$ & 75.0 & 42.0 & 1.0 & 90 & 18.2 & 140 \\
\hline $20: 13$ & $0: 57$ & 0.0 & 0.0 & 0.0 & 89 & 19.7 & 152 \\
\hline $20: 15$ & $0: 59$ & 74.0 & 41.0 & 0.7 & 89 & 19.7 & 152 \\
\hline $20: 17$ & $1: 01$ & 75.0 & 41.0 & 1.0 & 89 & 19.7 & 152 \\
\hline $20: 19$ & $1: 03$ & 75.0 & 42.0 & 1.0 & 90 & 17.5 & 135 \\
\hline $20: 21$ & $1: 05$ & 75.0 & 42.0 & 1.0 & 90 & 18.9 & 146 \\
\hline $20: 24$ & $1: 07$ & 75.0 & 41.0 & 1.0 & 90 & 18.5 & 143 \\
\hline $20: 26$ & 1:09 & 75.0 & 41.0 & 1.0 & 90 & 19.6 & 151 \\
\hline $20: 28$ & $1: 11$ & 75.0 & 41.0 & 1.0 & 90 & 20.2 & 156 \\
\hline $20: 30$ & $1: 13$ & 75.0 & 41.0 & 1.0 & 90 & 18.9 & 146 \\
\hline Average & & 75.0 & 41.4 & 1.0 & 89.6 & 19.1 & 1472 \\
\hline & & & & & & & \\
\hline Average & est Conditi & IS: SCRN2 & XLS & Feed & d Pressure & $=72.5$ psig & \\
\hline & & & & Concentrate & e Pressure & $=39.9 \mathrm{psig}$ & \\
\hline Date Run & 20-Apr-96 & & & & Feed Flow & $=1.0 \mathrm{gpm}$ & \\
\hline & & & & & Temp. & $=90 \mathrm{~F}$ & \\
\hline
\end{tabular}


SCRN2.XLS

\begin{tabular}{|c|c|c|c|c|c|c|c|}
\hline \multicolumn{8}{|l|}{ SCRN2 } \\
\hline \multicolumn{8}{|c|}{0.3 um $\mathrm{ZrO}_{2} / \mathrm{TiO}_{2}$} \\
\hline \multirow[t]{2}{*}{$\begin{array}{c}\text { TIME OF } \\
\text { DAY }\end{array}$} & $\begin{array}{c}\text { ELAPSED } \\
\text { TIME }\end{array}$ & $\begin{array}{c}\text { FEED } \\
\text { PRESSURE }\end{array}$ & $\begin{array}{c}\text { CONC } \\
\text { PRESSURE }\end{array}$ & $\begin{array}{c}\text { FEED FLOW } \\
\text { (L/min) }\end{array}$ & FEED TEMP & $\begin{array}{c}\text { PERMEATE } \\
\text { FLOW }\end{array}$ & $\begin{array}{l}\text { PERMEATE } \\
\text { FLUX }\end{array}$ \\
\hline & (HH:MM) & (psig) & (psig) & (gpm) & $\left({ }^{\circ} \mathrm{F}\right)$ & $(\mathrm{m} / \mathrm{min})$ & $\left(g a l / f^{2}-d a y\right)$ \\
\hline $19: 16$ & $0: 00$ & 75.0 & 42.0 & 1.0 & 90 & 21.4 & 165 \\
\hline $19: 18$ & $0: 01$ & 75.0 & 42.0 & 1.0 & 89 & 20.2 & 156 \\
\hline $19: 23$ & $0: 07$ & 75.0 & 42.0 & 1.0 & 89 & 20.4 & 157 \\
\hline $19: 24$ & $0: 08$ & .75 .0 & 42.0 & 1.0 & 89 & 20.4 & 157 \\
\hline $19: 27$ & $0: 11$ & 75.0 & 42.0 & 1.0 & 89 & 20.4 & 157 \\
\hline $19: 29$ & $0: 13$ & 75.0 & 42.0 & 1.0 & 89 & 25.0 & 193 \\
\hline $19: 31$ & $0: 15$ & 75.0 & 42.0 & 1.0 & 89 & 20.5 & 158 \\
\hline $19: 33$ & $0: 17$ & 75.0 & 42.0 & 1.0 & 90 & 24.0 & 185 \\
\hline $19: 35$ & $0: 19$ & 75.0 & 41.0 & 1.0 & 90 & 21.1 & 163 \\
\hline $19: 37$ & $0: 21$ & 75.0 & 42.0 & 1.0 & 90 & 18.6 & 144 \\
\hline $19: 39$ & $0: 23$ & 75.0 & 42.0 & 1.0 & 90 & 19.6 & 151 \\
\hline $19: 41$ & $0: 25$ & 75.0 & 41.0 & 1.0 & 90 & 19.1 & 147 \\
\hline $19: 43$ & $0: 27$ & 75.0 & 41.0 & 1.0 & 89 & 18.0 & 139 \\
\hline $19: 45$ & $0: 29$ & 75.0 & 42.0 & 1.0 & 89 & 18.0 & 139 \\
\hline $19: 47$ & $0: 31$ & 75.0 & 41.0 & 1.0 & 90 & 18.0 & 139 \\
\hline $19: 49$ & $0: 33$ & 75.0 & 41.0 & 1.0 & 89 & 18.6 & 144 \\
\hline $19: 51$ & $0: 35$ & 75.0 & 41.0 & 1.0 & 90 & 22.5 & 174 \\
\hline $19: 53$ & $0: 37$ & 75.0 & 41.0 & 1.0 & 90 & 18.4 & 142 \\
\hline $19: 55$ & $0: 39$ & 75.0 & 41.0 & 1.0 & 90 & 18.7 & 144 \\
\hline $19: 57$ & $0: 41$ & 75.0 & 41.0 & 1.0 & 90 & 18.5 & 143 \\
\hline $19: 59$ & $0: 43$ & 75.0 & 41.0 & 1.0 & 90. & 17.3 & 134 \\
\hline $20: 01$ & $0: 45$ & 75.0 & 41.0 & 1.0 & 89 & 19.6 & 151 \\
\hline $20: 03$ & $0: 47$ & 75.0 & 41.0 & 1.0 & 90 & 19.6 & 151 \\
\hline $20: 05$ & $0: 49$ & 75.0 & 41.0 & 1.0 & 90 & 17.8 & 137 \\
\hline $20: 07$ & $0: 51$ & 75.0 & 41.0 & 1.0 & 89 & 18.4 & 142 \\
\hline $20: 09$ & $0: 53$ & 75.0 & 42.0 & 1.0 & 90 & 17.9 & 138 \\
\hline $20: 11$ & $0: 55$ & 75.0 & 42.0 & 1.0 & 90 & 19.1 & 147 \\
\hline $20: 13$ & $0: 57$ & 0.0 & 0.0 & 0.0 & 89 & 19.1 & 147 \\
\hline $20: 15$ & $0: 59$ & 74.0 & 41.0 & 0.7 & 89 & 19.1 & 147 \\
\hline $20: 17$ & $1: 01$ & 75.0 & 41.0 & 1.0 & 89 & 21.8 & 168 \\
\hline $20: 19$ & $1: 03$ & 75.0 & 42.0 & 1.0 & 90 & 19.7 & 152 \\
\hline $20: 21$ & $1: 05$ & 75.0 & 42.0 & 1.0 & 90 & 19.3 & 149 \\
\hline $20: 24$ & $1: 07$ & 75.0 & 41.0 & 1.0 & 90 & 18.9 & 146 \\
\hline $20: 26$ & $1: 09$ & 75.0 & 41.0 & 1.0 & 90 & 20.1 & 155 \\
\hline $20: 28$ & $1: 11$ & 75.0 & 41.0 & 1.0 & 90 & 20.1 & 155 \\
\hline $20: 30$ & $1: 13$ & 75.0 & 41.0 & 1.0 & 90 & 18.9 & 146 \\
\hline & & & & & & & \\
\hline Average & & 75.0 & 41.4 & 1.0 & 89.6 & 19.7 & 151.9 \\
\hline & & & & & & & \\
\hline Average & est Conditi & ns: SCRN & $X \mathrm{XLS}$ & Fee & ed Pressure & $=72.5 \mathrm{psig}$ & \\
\hline & & & & Concentrat & te Pressure & $=39.9 \mathrm{psig}$ & \\
\hline Date Run & 20-Apr-96 & & & & Feed Flow & $=1.0 \mathrm{gpm}$ & \\
\hline & & & & & Temp. & $=90 \mathrm{~F}$ & \\
\hline
\end{tabular}




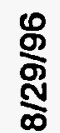

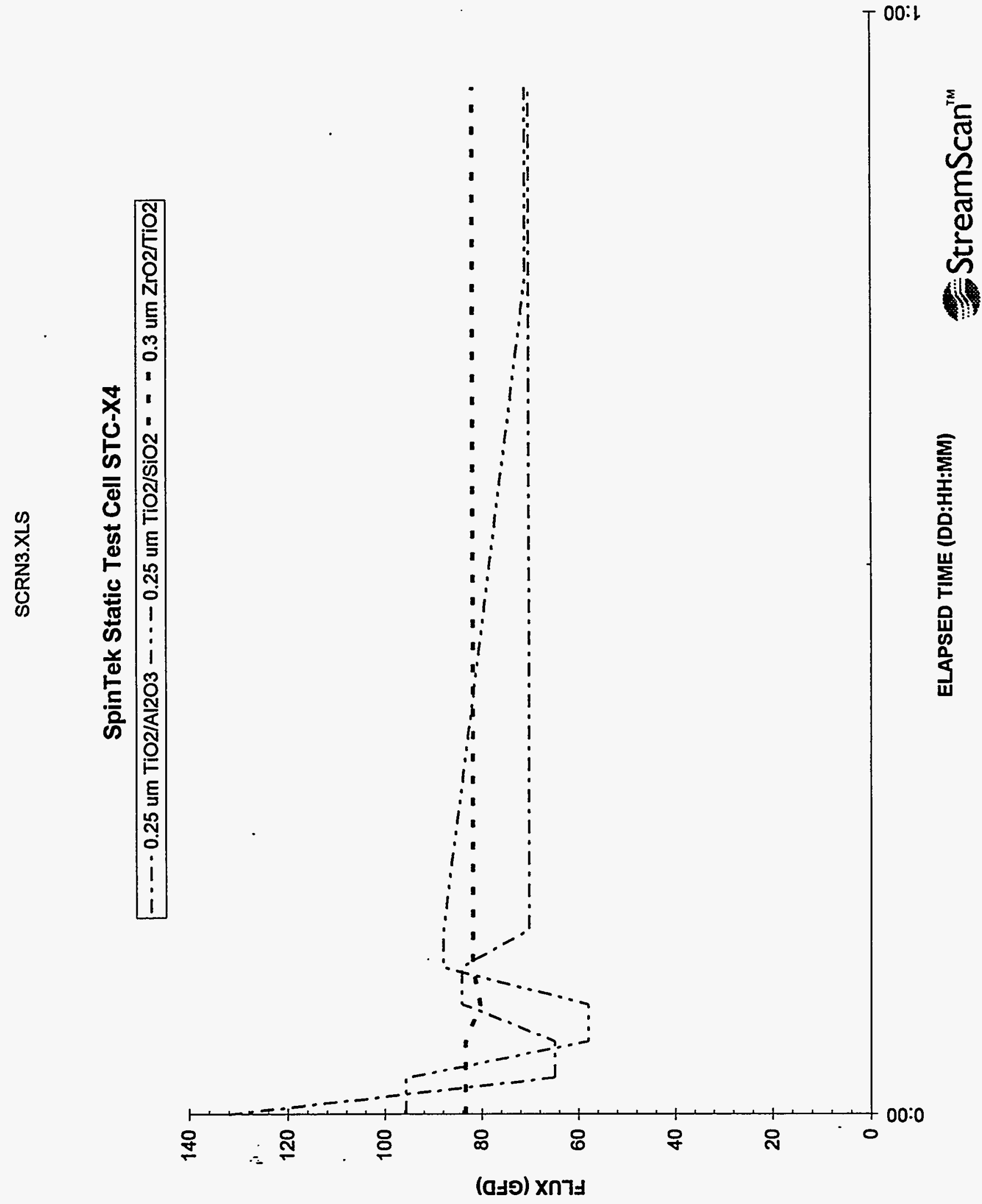


SCRN3.XLS

\begin{tabular}{|c|c|c|c|c|c|c|c|}
\hline SCRN3 & & & & & & & \\
\hline 0.25 um & $\mathrm{TiO}_{2} / \mathrm{A}$ & $\mathrm{I}_{2} \mathrm{O}_{3}$ & & & & & \\
\hline $\begin{array}{c}\text { TIME OF } \\
\text { DAY }\end{array}$ & $\begin{array}{l}\text { ELAPSED } \\
\text { TIME }\end{array}$ & $\begin{array}{c}\text { FEED } \\
\text { PRESSURE }\end{array}$ & $\begin{array}{c}\text { CONC } \\
\text { PRESSURE }\end{array}$ & $\begin{array}{c}\text { FEED FLOW } \\
\text { (L/min) }\end{array}$ & FEED TEMP & $\begin{array}{c}\text { PERMEATE } \\
\text { FLOW }\end{array}$ & $\begin{array}{l}\text { PERMEATE } \\
\text { FLUX }\end{array}$ \\
\hline & (HH:MM) & (psig) & (psig) & (gpm) & $\left({ }^{\circ} \mathrm{F}\right)$ & (ml/min) & (gal/fta -day) \\
\hline $20: 41$ & $0: 00$ & 46.0 & 16.0 & 1.0 & 90 & 17.1 & 132 \\
\hline $20: 43$ & $0: 02$ & 35.0 & 14.0 & 0.8 & 90 & 8.4 & 65 \\
\hline $20: 45$ & $0: 04$ & 50.0 & 16.0 & 1.0 & 89 & 8.4 & 65 \\
\hline $20: 47$ & $0: 06$ & 54.0 & 16.0 & 1.1 & 89 & 10.9 & 84 \\
\hline $20: 49$ & $0: 08$ & 48.0 & 15.0 & 1.0 & 90 & 10.9 & 84 \\
\hline $20: 51$ & $0: 10$ & 47.0 & 14.0 & 1.0 & 89 & 9.1 & 70 \\
\hline $21: 27$ & $0: 45$ & 47.0 & 14.0 & 1.0 & 90 & 9.1 & 70 \\
\hline $21: 34$ & $0: 53$ & 47.0 & 14.0 & 1.0 & 90 & 9.1 & 70 \\
\hline $21: 37$ & $0: 56$ & 47.0 & 14.0 & 1.0 & 90 & 9.1 & 70 \\
\hline Average & & 46.8 & 14.8 & 1.0 & 89.7 & 10.2 & 79.0 \\
\hline Average T & est Conditi & ns: SCRN3 &.$X L S$ & Fee & d Pressure & $=46.9 \mathrm{psig}$ & \\
\hline & & & & Concentra & te Pressure & $=14.6 \mathrm{psig}$ & \\
\hline Date Run: & 20-Apr-96 & & & & Feed Flow & $=1.0 \mathrm{gpm}$ & \\
\hline & & & & & Temp. $=$ & $=90 \mathrm{~F}$ & \\
\hline
\end{tabular}




\begin{tabular}{|c|c|c|c|c|c|c|c|}
\hline SCRN3 & & & & & & & \\
\hline \multicolumn{8}{|c|}{0.25 um $\mathrm{TiO}_{2} / \mathrm{SiO}_{2}$} \\
\hline \multirow[t]{2}{*}{$\begin{array}{c}\text { TIME OF } \\
\text { DAY }\end{array}$} & $\begin{array}{l}\text { ELAPSED } \\
\text { TIME }\end{array}$ & $\begin{array}{c}\text { FEED } \\
\text { PRESSURE }\end{array}$ & $\begin{array}{c}\text { CONC } \\
\text { PRESSURE }\end{array}$ & $\begin{array}{c}\text { FEED FLOW } \\
\text { (L'min) }\end{array}$ & FEED TEMP & $\begin{array}{c}\text { PERMEATE } \\
\text { FLOW }\end{array}$ & $\begin{array}{l}\text { PERMEATE } \\
\text { FLUX }\end{array}$ \\
\hline & (HH:MM) & (psig) & (psig) & (gpm) & $\left({ }^{\circ} \mathrm{F}\right)$ & (mU/min) & (gal/fiteday) \\
\hline $20: 41$ & $0: 00$ & 46.0 & 16.0 & 1.0 & 90 & 12.4 & 96 \\
\hline $20: 43$ & $0: 01$ & 35.0 & 14.0 & 0.8 & 90 & 12.4 & 96 \\
\hline $20: 45$ & $0: 03$ & 50.0 & 16.0 & 1.0 & 89 & 7.5 & 58 \\
\hline $20: 47$ & $0: 06$ & 54.0 & 16.0 & 1.1 & 89 & 7.5 & 58 \\
\hline $20: 49$ & $0: 08$ & 48.0 & 15.0 & 1.0 & 90 & 11.4 & 88 \\
\hline $20: 51$ & $0: 10$ & 47.0 & 14.0 & 1.0 & 89 & 11.4 & 88 \\
\hline $21: 27$ & $0: 45$ & 47.0 & 14.0 & 1.0 & 90 & 9.2 & 71 \\
\hline $21: 34$ & $0: 53$ & 47.0 & 14.0 & 1.0 & 90 & 9.2 & 71 \\
\hline 21:37 & $0: 56$ & 47.0 & 14.0 & 1.0 & 90 & 9.2 & 71 \\
\hline Average & & 46.8 & 14.8 & 1.0 & 89.7 & 10.0 & 77.3 \\
\hline & & & & \\
\hline & & & & \multicolumn{3}{|c|}{ Feed Pressure $=46.9$ psig } & \\
\hline Date Run: & 20-Apr-96 & & & \multicolumn{3}{|c|}{ Feed Flow $=1.0 \mathrm{gpm}$} & \\
\hline & & & & \multicolumn{3}{|c|}{ Temp. $=90 \mathrm{~F}$} & \\
\hline
\end{tabular}


SCRN3.XLS

\begin{tabular}{|c|c|c|c|c|c|c|c|}
\hline SCRN3 & & & & & & & \\
\hline $0.3 \mathrm{um}$ & $\mathrm{ZrO}_{2} / \mathrm{Ti}$ & & & & & & \\
\hline $\begin{array}{c}\text { TIME OF } \\
\text { DAY }\end{array}$ & $\begin{array}{c}\text { ELAPSED } \\
\text { TIME }\end{array}$ & $\begin{array}{c}\text { FEED } \\
\text { PRESSURE }\end{array}$ & $\begin{array}{c}\text { CONC } \\
\text { PRESSURE }\end{array}$ & $\begin{array}{c}\text { FEED FLOW } \\
\text { (Umin) }\end{array}$ & FEED TEMP & $\begin{array}{l}\text { PERMEATE } \\
\text { FLOW }\end{array}$ & $\begin{array}{l}\text { PERMEATE } \\
\text { FLUX }\end{array}$ \\
\hline & (HH:MM) & (psig) & (psig) & (gpm) & $\left(0^{\circ} \mathrm{F}\right)$ & (m/min) & (gal/ft'-day) \\
\hline $20: 41$ & $0: 00$ & 46.0 & 16.0 & 1.0 & 90 & 10.8 & 83 \\
\hline $20: 43$ & $0: 01$ & 35.0 & 14.0 & 0.8 & 90 & 10.8 & 83 \\
\hline $20: 45$ & $0: 03$ & 50.0 & 16.0 & 1.0 & 89 & 10.8 & 83 \\
\hline $20: 47$ & $0: 06$ & 54.0 & 16.0 & 1.1 & 89 & 10.4 & 80 \\
\hline $20: 49$ & $0: 08$ & 48.0 & 15.0 & 1.0 & 90 & 10.6 & 82 \\
\hline $20: 51$ & $0: 10$ & 47.0 & 14.0 & 1.0 & 89 & 10.6 & 82 \\
\hline $21: 27$ & $0: 45$ & 47.0 & 14.0 & 1.0 & 90 & 10.6 & 82 \\
\hline $21: 34$ & $0: 53$ & 47.0 & 14.0 & 1.0 & 90 & 10.6 & 82 \\
\hline $21: 37$ & $0: 56$ & 47.0 & 14.0 & 1.0 & 90 & 10.6 & 82 \\
\hline Average & & 46.8 & 14.8 & 1.0 & 89.7 & 10.6 & 82.1 \\
\hline Average $T e$ & st Conditic & ns: SCRN3 & XLS & Feed & Pressure $=$ & $=46.9 \mathrm{psig}$ & \\
\hline & & & & Concentrat & Pressure & $=14.6 \mathrm{psig}$ & \\
\hline Date Run: & $20-A p r-96$ & & & & Feed Flow = & $=1.0 \mathrm{gpm}$ & \\
\hline & & & & & Temp. $=$ & $90 \mathrm{~F}$ & \\
\hline
\end{tabular}




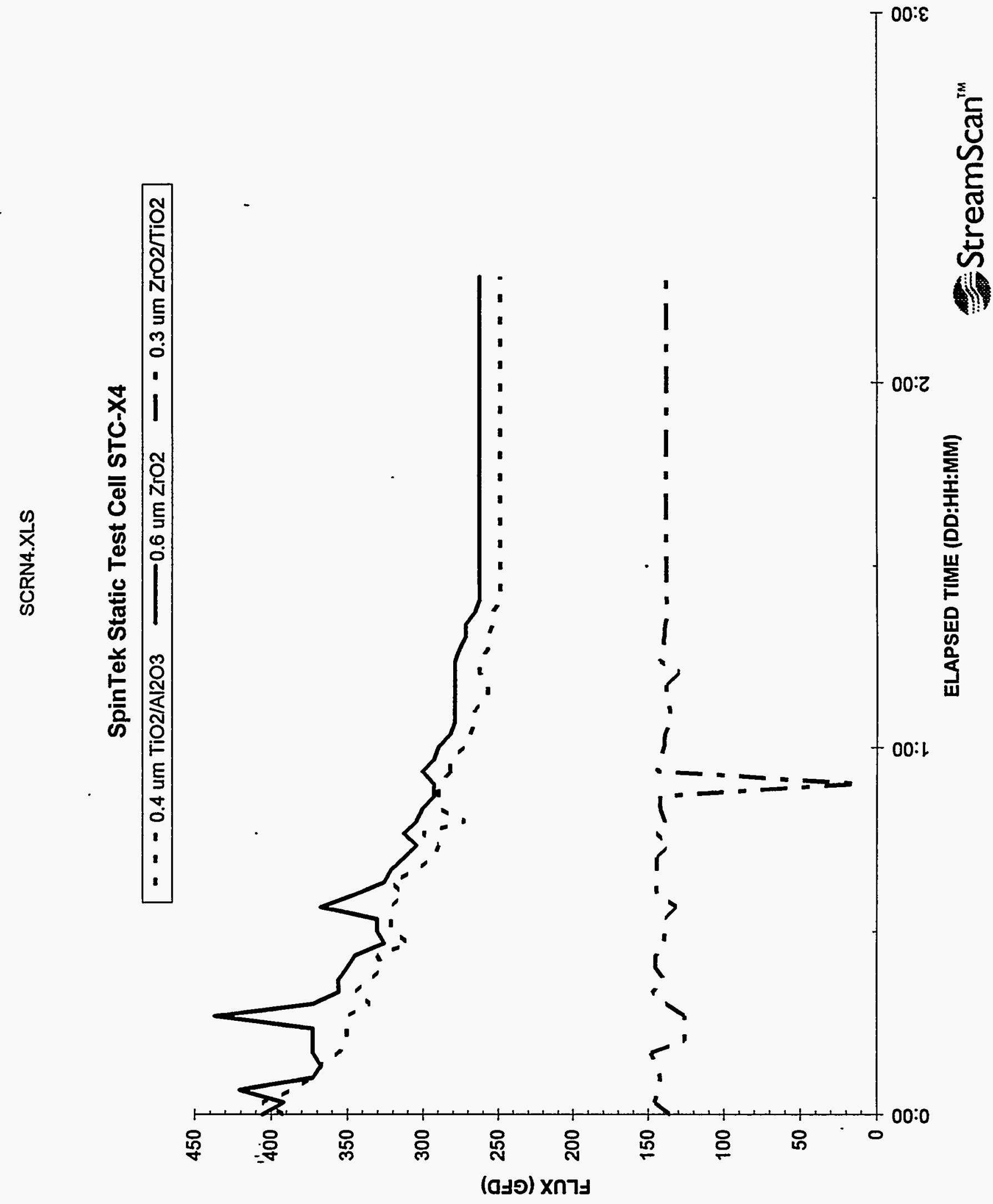


SCRN4.XLS

\begin{tabular}{|c|c|c|c|c|c|c|c|}
\hline SCRN4 & & & & & & & \\
\hline $0.4 \mathrm{um}$ & $\mathrm{IO}_{2} / \mathrm{Al}$ & & & & & & \\
\hline $\begin{array}{c}\text { TIME OF } \\
\text { DAY }\end{array}$ & $\begin{array}{c}\text { ELAPSED } \\
\text { TIME }\end{array}$ & $\begin{array}{c}\text { FEED } \\
\text { PRESSURE }\end{array}$ & $\begin{array}{c}\text { CONC } \\
\text { PRESSURE }\end{array}$ & $\begin{array}{c}\text { FEED FLOW } \\
\text { (L/min) }\end{array}$ & FEED TEMP & $\begin{array}{c}\text { PERMEATE } \\
\text { FLOW }\end{array}$ & $\begin{array}{l}\text { PERMEATE } \\
\text { FLUX }\end{array}$ \\
\hline & (HH:MM) & (psig) & (psig) & (gpm) & $\left({ }^{\circ} \mathrm{F}\right)$ & (mU/min) & (gal/ft'tay) \\
\hline $10: 01$ & $0: 00$ & 75.0 & 40.0 & 1.1 & 89 & 50.8 & 392 \\
\hline $10: 03$ & $0: 02$ & 75.0 & 40.0 & 1.1 & 89 & 52.6 & 406 \\
\hline $10: 05$ & $0: 04$ & 75.0 & 40.0 & 1.1 & 90 & 50.0 & 386 \\
\hline $10: 07$ & $0: 06$ & 74.0 & 40.0 & 1.1 & 90 & 48.3 & 373 \\
\hline $10: 09$ & $0: 08$ & 75.0 & 40.0 & 1.1 & 90 & 47.6 & 367 \\
\hline $10: 11$ & $0: 10$ & 75.0 & 40.0 & 1.1 & 89 & 46.1 & 356 \\
\hline $10: 13$ & $0: 12$ & 74.0 & 40.0 & 1.1 & 90 & 45.4 & 350 \\
\hline $10: 15$ & $0: 14$ & 0.0 & 0.0 & 0.0 & 90 & 45.4 & 350 \\
\hline $10: 17$ & $0: 16$ & 75.0 & 40.0 & 1.1 & 89 & 45.4 & 350 \\
\hline $10: 19$ & $0: 18$ & 75.0 & 40.0 & 1.1 & 89 & 43.4 & 335 \\
\hline $10: 21$ & $0: 20$ & 74.0 & 40.0 & 1.1 & 90 & 44.7 & 345 \\
\hline $10: 23$ & $0: 22$ & 75.0 & 40.0 & 1.1 & 90 & 43.4 & 335 \\
\hline $10: 25$ & $0: 24$ & 74.0 & 40.0 & 1.1 & 90 & 42.2 & 326 \\
\hline $10: 27$ & $0: 26$ & 74.0 & 40.0 & 1.1 & 90 & 42.8 & 330 \\
\hline $10: 29$ & $0: 28$ & 74.0 & 40.0 & 1.1 & 90 & 40.0 & 309 \\
\hline $10: 31$ & $0: 30$ & 0.0 & 0.0 & 0.2 & 89 & 41.6 & 321 \\
\hline $10: 33$ & $0: 32$ & 0.0 & 0.0 & 0.0 & 89 & 41.6 & 321 \\
\hline $10: 35$ & $0: 34$ & 75.0 & 40.0 & 1.1 & 89 & 41.6 & 321 \\
\hline $10: 37$ & $0: 36$ & 75.0 & 40.0 & 1.1 & 90 & 40.5 & 313 \\
\hline $10: 39$ & $0: 38$ & 74.0 & 40.0 & 1.1 & 90 & 41.6 & 321 \\
\hline $10: 41$ & $0: 40$ & 75.0 & 40.0 & 1.1 & 90 & 39.4 & 304 \\
\hline $10: 43$ & $0: 42$ & 75.0 & 40.0 & 1.1 & 89 & 37.9 & 292 \\
\hline $10: 45$ & $0: 44$ & 75.0 & 40.0 & 1.1 & 89 & 37.5 & 289 \\
\hline $10: 47$ & $0: 46$ & 75.0 & 41.0 & 1.1 & 90 & 38.9 & 300 \\
\hline $10: 49$ & $0: 48$ & 75.0 & 40.0 & 1.1 & 90 & 35.2 & 272 \\
\hline $10: 51$ & $0: 50$ & 75.0 & 41.0 & 1.1 & 89 & 37.5 & 289 \\
\hline $10: 53$ & $0: 52$ & 0.0 & 0.0 & 0.0 & 89 & 37.5 & 289 \\
\hline $10: 55$ & $0: 54$ & 75.0 & 41.0 & 1.1 & 89 & 37.5 & 289 \\
\hline $10: 57$ & $0: 56$ & 75.0 & 40.0 & 1.1 & 90 & 36.5 & 282 \\
\hline $10: 59$ & $.0: 58$ & 75.0 & 41.0 & 1.1 & 89 & 36.5 & 282 \\
\hline $11: 01$ & $1: 00$ & 75.0 & 40.0 & 1.1 & 90 & 35.2 & 272 \\
\hline $11: 03$ & $1: 02$ & 75.0 & 41.0 & 1.1 & 89 & 34.8 & 269 \\
\hline $11: 05$ & $1: 04$ & 75.0 & 40.0 & 1.1 & 90 & 34.4 & 265 \\
\hline $11: 07$ & $1: 06$ & 75.0 & 40.0 & 1.1 & 89 & 34.4 & 265 \\
\hline $11: 10$ & $1: 08$ & 0.0 & 0.0 & 0.0 & 89 & 33.3 & 257 \\
\hline $11: 12$ & $1: 10$ & 75.0 & 40.0 & 1.1 & 88 & 33.3 & 257 \\
\hline $11: 14$ & $1: 12$ & 75.0 & 41.0 & 1.1 & 90 & 34.0 & 262 \\
\hline $11: 16$ & $1: 14$ & 75.0 & 40.0 & 1.1 & 89 & 34.0 & 262 \\
\hline $11: 18$ & $1: 16$ & 75.0 & 41.0 & 1.1 & 90 & 33.3 & 257 \\
\hline $11: 20$ & $1: 18$ & 75.0 & 40.0 & 1.1 & 90 & 33.3 & 257 \\
\hline $11: 22$ & $1: 20$ & 75.0 & 40.0 & 1.1 & 90 & 32.9 & 254 \\
\hline $11: 24$ & $1: 22$ & 75.0 & 41.0 & 1.1 & 89 & 32.9 & 254 \\
\hline $11: 26$ & $.1: 24$ & 75.0 & 40.0 & 1.1 & 90 & 32.2 & 248 \\
\hline $11: 57$ & $-1: 56$ & 75.0 & 40.0 & 1.1 & 90 & 32.2 & 248 \\
\hline
\end{tabular}


SCRN4.XLS

\begin{tabular}{|c|c|c|c|c|c|c|c|}
\hline \multicolumn{8}{|c|}{$0.4 \mathrm{um} \mathrm{TiO}_{2} / \mathrm{Al}_{2} \mathrm{O}_{3}$} \\
\hline \multirow[t]{2}{*}{$\begin{array}{c}\text { TIME OF } \\
\text { DAY }\end{array}$} & $\begin{array}{l}\text { ELAPSED } \\
\text { TIME }\end{array}$ & $\begin{array}{c}\text { FEED } \\
\text { PRESSURE }\end{array}$ & $\begin{array}{c}\text { CONC } \\
\text { PRESSURE }\end{array}$ & $\begin{array}{l}\text { FEED FLOW } \\
\text { (L/min) }\end{array}$ & FEED TEMP & $\begin{array}{c}\text { PERMEATE } \\
\text { FLOW }\end{array}$ & $\begin{array}{l}\text { PERMEATE } \\
\text { FLUX }\end{array}$ \\
\hline & (HH:MM) & (psig) & (psig) & (gpm) & $\left({ }^{\circ} \mathrm{F}\right)$ & $(\mathrm{ml} / \mathrm{min})$ & (gal/fteday) \\
\hline $11: 58$ & $1: 56$ & 75.0 & 40.0 & 1.1 & 90 & 32.2 & 248 \\
\hline $12: 00$ & $1: 59$ & 75.0 & 40.0 & 1.1 & 90 & 32.2 & 248 \\
\hline $12: 02$ & $2: 01$ & 75.0 & 40.0 & 1.1 & 90 & 32.2 & 248 \\
\hline $12: 05$ & $2: 03$ & 75.0 & 40.0 & 1.1 & 90 & 32.2 & 248 \\
\hline $12: 06$ & $2: 04$ & 75.0 & 40.0 & 1.1 & 90 & 32.2 & 248 \\
\hline $12: 09$ & $2: 08$ & 75.0 & 40.0 & 1.1 & 90 & 32.2 & 248 \\
\hline $12: 11$ & $2: 09$ & 75.0 & 40.0 & 1.1 & 90 & 32.2 & 248 \\
\hline $12: 15$ & $2: 14$ & 75.0 & 40.0 & 1.1 & 90 & 32.2 & 248 \\
\hline $12: 19$ & $2: 17$ & 75.0 & 40.0 & 1.1 & 90 & 32.2 & 248 \\
\hline Average & & 74.9 & 40.2 & 1.1 & 89.6 & 38.1 & 294.2 \\
\hline \multirow{2}{*}{\multicolumn{4}{|c|}{ Average Test Conditions: SCRN4.XLS }} & & & & \\
\hline & & & & \multicolumn{4}{|c|}{ Feed Pressure $=72.9 \mathrm{psig}$} \\
\hline & & & & \multicolumn{4}{|c|}{ Concentrate Pressure $=39.1 \mathrm{psig}$} \\
\hline \multirow{2}{*}{\multicolumn{2}{|c|}{ Date Run: 21-Apr-96 }} & & & \multirow{2}{*}{\multicolumn{3}{|c|}{$\begin{aligned} \text { Feed Flow } & =1.0 \mathrm{gpm} \\
\text { Temp } & =90 \mathrm{~F}\end{aligned}$}} & \\
\hline & & & & & & & \\
\hline
\end{tabular}


SCRN4.XLS

\begin{tabular}{|c|c|c|c|c|c|c|c|}
\hline SCRN4 & & & & & & & \\
\hline $0.6 \mathrm{um}$ & $\mathrm{ZrO}_{2}$ & & & & & & \\
\hline $\begin{array}{c}\text { TIME OF } \\
\text { DAY }\end{array}$ & $\begin{array}{l}\text { ELAPSED } \\
\text { TIME }\end{array}$ & $\begin{array}{c}\text { FEED } \\
\text { PRESSURE }\end{array}$ & $\begin{array}{c}\text { CONC } \\
\text { PRESSURE }\end{array}$ & $\begin{array}{c}\text { FEED FLOW } \\
\text { (L'min) }\end{array}$ & FEED TEMP & $\begin{array}{l}\text { PERMEATE } \\
\text { FLOW }\end{array}$ & $\begin{array}{l}\text { PERMEATE } \\
\text { FLUX }\end{array}$ \\
\hline & (HH:MM) & (psig) & (psig) & (gpm) & $\left({ }^{\circ} \mathrm{F}\right)$ & $(\mathrm{m} / \mathrm{min})$ & $\left(g a l / f^{2}-d a y\right)$ \\
\hline $10: 01$ & $0: 00$ & 75.0 & 40.0 & 1.1 & 89 & 52.6 & 406 \\
\hline $10: 03$ & $0: 02$ & 75.0 & 40.0 & 1.1 & 89 & 50.8 & 392 \\
\hline $10: 05$ & $0: 04$ & 75.0 & 40.0 & 1.1 & 90 & 54.5 & 421 \\
\hline $10: 07$ & $0: 06$ & 74.0 & 40.0 & 1.1 & 90 & 48.3 & 373 \\
\hline $10: 09$ & $0: 08$ & 75.0 & 40.0 & 1.1 & 90 & 47.6 & 367 \\
\hline $10: 11$ & $0: 10$ & 75.0 & 40.0 & 1.1 & 89 & 48.3 & 373 \\
\hline $10: 13$ & $0: 12$ & 74.0 & 40.0 & 1.1 & 90 & 48.3 & 373 \\
\hline $10: 15$ & $0: 14$ & 0.0 & 0.0 & 0.0 & 90 & 48.3 & 373 \\
\hline $10: 17$ & $0: 16$ & 75.0 & 40.0 & 1.1 & 89 & 56.6 & 437 \\
\hline $10: 19$ & $0: 18$ & 75.0 & 40.0 & 1.1 & 89 & 48.3 & 373 \\
\hline $10: 21$ & $0: 20$ & 74.0 & 40.0 & 1.1 & 90 & 46.1 & 356 \\
\hline $10: 23$ & $0: 22$ & 75.0 & 40.0 & 1.1 & 90 & 46.1 & 356 \\
\hline $10: 25$ & $0: 24$ & 74.0 & 40.0 & 1.1 & 90 & 45.4 & 350 \\
\hline $10: 27$ & $0: 26$ & 74.0 & 40.0 & 1.1 & 90 & 44.7 & 345 \\
\hline $10: 29$ & $0: 28$ & 74.0 & 40.0 & 1.1 & 90 & 42.2 & 326 \\
\hline $10: 31$ & $0: 30$ & 0.0 & 0.0 & 0.2 & 89 & 42.8 & 330 \\
\hline $10: 33$ & $0: 32$ & 0.0 & 0.0 & 0.0 & 89 & 42.8 & 330 \\
\hline $10: 35$ & $0: 34$ & 75.0 & 40.0 & 1.1 & 89 & 47.6 & 367 \\
\hline $10: 37$ & $0: 36$ & 75.0 & 40.0 & 1.1 & 90 & 44.7 & 345 \\
\hline $10: 39$ & $0: 38$ & 74.0 & 40.0 & 1.1 & 90 & 42.2 & 326 \\
\hline $10: 41$ & $0: 40$ & 75.0 & 40.0 & 1.1 & 90 & 41.6 & 321 \\
\hline $10: 43$ & $0: 42$ & 75.0 & 40.0 & 1.1 & 89 & 40.5 & 313 \\
\hline $10: 45$ & $0: 44$ & 75.0 & 40.0 & 1.1 & 89 & 39.4 & 304 \\
\hline $10: 47$ & $0: 46$ & 75.0 & 41.0 & 1.1 & 90 & 40.5 & 313 \\
\hline $10: 49$ & $0: 48$ & 75.0 & 40.0 & 1.1 & 90 & 39.4 & 304 \\
\hline $10: 51$ & $0: 50$ & 75.0 & 41.0 & 1.1 & 89 & 38.9 & 300 \\
\hline $10: 53$ & $0: 52$ & 0.0 & 0.0 & 0.0 & 89 & 37.9 & 292 \\
\hline $10: 55$ & $0: 54$ & 75.0 & 41.0 & 1.1 & 89 & 37.9 & 292 \\
\hline $10: 57$ & $0: 56$ & 75.0 & 40.0 & 1.1 & 90 & 38.9 & 300 \\
\hline $10: 59$ & $0: 58$ & 75.0 & 41.0 & 1.1 & 89 & 37.9 & 292 \\
\hline $11: 02$ & $1: 00$ & 75.0 & 40.0 & 1.1 & 90 & 37.5 & 289 \\
\hline $11: 04$ & $1: 02$ & 75.0 & 41.0 & 1.1 & 89 & 36.5 & 282 \\
\hline $11: 06$ & 1:04 & 75.0 & 40.0 & 1.1 & 90 & 36.1 & 279 \\
\hline $11: 08$ & $1: 06$ & 75.0 & 40.0 & 1.1 & 89 & 36.1 & 279 \\
\hline $11: 10$ & $1: 08$ & 0.0 & 0.0 & 0.0 & 89 & 36.1 & 279 \\
\hline $11: 12$ & $1: 10$ & 75.0 & 40.0 & 1.1 & 88 & 36.1 & 279 \\
\hline $11: 14$ & $1: 12$ & 75.0 & 41.0 & 1.1 & 90 & 36.1 & 279 \\
\hline $11: 16$ & $1: 14$ & 75.0 & 40.0 & 1.1 & 89 & 36.1 & 279 \\
\hline $11: 18$ & $1: 16$ & 75.0 & 41.0 & 1.1 & 90 & 35.7 & 275 \\
\hline $11: 20$ & $1: 18$ & 75.0 & 40.0 & 1.1 & 90 & 35.2 & 272 \\
\hline $11: 22$ & $1: 20$ & 75.0 & 40.0 & 1.1 & 90 & 35.2 & 272 \\
\hline $11: 24$ & $1: 22$ & 75.0 & 41.0 & 1.1 & 89 & 34.4 & 265 \\
\hline $11: 26$ & $1: 24$ & 75.0 & 40.0 & 1.1 & 90 & 34.0 & 262 \\
\hline $11: 57$ & $-1: 56$ & 75.0 & 40.0 & 1.1 & 90 & 34.0 & 262 \\
\hline
\end{tabular}


SCRN4.XLS

\begin{tabular}{|c|c|c|c|c|c|c|c|}
\hline \multicolumn{8}{|c|}{0.6 um $\mathrm{ZrO}_{2}$} \\
\hline \multirow[t]{2}{*}{$\begin{array}{l}\text { TIME OF } \\
\text { DAY }\end{array}$} & $\begin{array}{l}\text { ELAPSED } \\
\text { TIME }\end{array}$ & $\begin{array}{c}\text { FEED } \\
\text { PRESSURE }\end{array}$ & $\begin{array}{c}\text { CONC } \\
\text { PRESSURE }\end{array}$ & $\begin{array}{c}\text { FEED FLOW } \\
\text { (L/min) }\end{array}$ & FEED TEMP & $\begin{array}{l}\text { PERMEATE } \\
\text { FLOW }\end{array}$ & $\begin{array}{l}\text { PERMEATE } \\
\text { FLUX }\end{array}$ \\
\hline & (HH:MM) & (psig) & (psig) & (gpm) & $\left({ }^{\circ} \mathrm{F}\right)$ & $(\mathrm{m} / \mathrm{min})$ & (gal/f/2 -day) \\
\hline $11: 58$ & $1: 56$ & 75.0 & 40.0 & 1.1 & 90 & 34.0 & 262 \\
\hline $12: 00$ & $1: 58$ & 75.0 & 40.0 & 1.1 & 90 & 34.0 & 262 \\
\hline $12: 02$ & $2: 01$ & 75.0 & 40.0 & 1.1 & 90 & 34.0 & 262 \\
\hline $12: 05$ & $2: 03$ & 75.0 & 40.0 & 1.1 & 90 & 34.0 & 262 \\
\hline $12: 06$ & $2: 04$ & 75.0 & 40.0 & 1.1 & 90 & 34.0 & 262 \\
\hline $12: 09$ & $2: 08$ & 75.0 & 40.0 & 1.1 & 90 & 34.0 & 262 \\
\hline $12: 11$ & $2: 09$ & 75.0 & 40.0 & 1.1 & 90 & 34.0 & 262 \\
\hline $12: 15$ & $2: 14$ & 75.0 & 40.0 & 1.1 & 90 & 34.0 & 262 \\
\hline $12: 19$ & $2: 17$ & 75.0 & 40.0 & 1.1 & 90 & 34.0 & 262 \\
\hline & & & & & & & \\
\hline $\begin{array}{l}\text { Average } \\
\end{array}$ & & 74.9 & 40.2 & 1.1 & 89.6 & 40.4 & 311.6 \\
\hline \multicolumn{4}{|c|}{$\frac{1}{\mid \text { Average Test Conditions: SCRN4.XLS }}$} & \multicolumn{4}{|c|}{$\frac{1}{\text { Feed Pressure }}=72.9 \mathrm{psig}$} \\
\hline \multirow{2}{*}{\multicolumn{2}{|c|}{\begin{tabular}{|l|l|} 
& \\
Date Run: 21-Apr-96 \\
\end{tabular}}} & & & \multicolumn{4}{|c|}{ Concentrate Pressure $=39.1 \mathrm{psig}$} \\
\hline & & & & \multirow{2}{*}{\multicolumn{3}{|c|}{$\begin{array}{c}\text { Feed Flow }=1.0 \mathrm{gpm} \\
\text { Temp. }=90 \mathrm{~F}\end{array}$}} & \\
\hline & & & & & & & \\
\hline
\end{tabular}


SCRN4.XLS

\begin{tabular}{|c|c|c|c|c|c|c|c|}
\hline SCRN4 & & & & & & & \\
\hline $0.3 \mathrm{um}$ & $\mathrm{ZrO}_{2} / \mathrm{Ti}$ & & & & & & \\
\hline $\begin{array}{c}\text { TIME OF } \\
\text { DAY }\end{array}$ & $\begin{array}{c}\text { ELAPSED } \\
\text { TIME }\end{array}$ & $\begin{array}{c}\text { FEED } \\
\text { PRESSURE }\end{array}$ & $\begin{array}{c}\text { CONC } \\
\text { PRESSURE }\end{array}$ & $\begin{array}{c}\text { FEED FLOW } \\
\text { (Lmin) }\end{array}$ & FEED TEMP & $\begin{array}{l}\text { PERMEATE } \\
\text { FLOW }\end{array}$ & $\begin{array}{l}\text { PERMEATE } \\
\text { FLUX }\end{array}$ \\
\hline & (HH:MM) & (psig) & (psig) & (gpm) & $\left({ }^{\circ} \mathrm{F}\right)$ & $(\mathrm{ml} / \mathrm{min})$ & (gal/ft $\left.t^{2}-d a y\right)$ \\
\hline $10: 01$ & $0: 00$ & 75.0 & 40.0 & 1.1 & 89 & 17.6 & 136 \\
\hline $10: 03$ & $0: 02$ & 75.0 & 40.0 & 1.1 & 89 & 18.9 & 146 \\
\hline $10: 05$ & 0:04 & 75.0 & 40.0 & 1.1 & 90 & 18.6 & 144 \\
\hline $10: 07$ & $0: 06$ & 74.0 & 40.0 & 1.1 & 90 & 18.4 & 142 \\
\hline $10: 09$ & $0: 08$ & 75.0 & 40.0 & 1.1 & 90 & 18.7 & 144 \\
\hline $10: 11$ & $0: 10$ & 75.0 & 40.0 & 1.1 & 89 & 19.2 & 148 \\
\hline $10: 13$ & $0: 12$ & 74.0 & 40.0 & 1.1 & 90 & 16.3 & 126 \\
\hline $10: 15$ & $0: 14$ & 0.0 & 0.0 & 0.0 & 90 & 16.3 & 126 \\
\hline $10: 17$ & $0: 16$ & 75.0 & 40.0 & 1.1 & 89 & 16.3 & 126 \\
\hline $10: 19$ & $0: 18$ & 75.0 & 40.0 & 1.1 & 89 & 17.9 & 138 \\
\hline $10: 21$ & $0: 20$ & 74.0 & 40.0 & 1.1 & 90 & 19.1 & 147 \\
\hline $10: 23$ & $0: 22$ & 75.0 & 40.0 & 1.1 & 90 & 18.1 & 140 \\
\hline $10: 25$ & $0: 24$ & 74.0 & 40.0 & 1.1 & 90 & 18.8 & 145 \\
\hline $10: 27$ & $0: 26$ & 74.0 & 40.0 & 1.1 & 90 & 18.8 & 145 \\
\hline $10: 29$ & $0: 28$ & 74.0 & 40.0 & 1.1 & 90 & 18.1 & 140 \\
\hline $10: 31$ & $0: 30$ & 0.0 & 0.0 & 0.2 & 89 & 18.0 & 139 \\
\hline $10: 33$ & $0: 32$ & 0.0 & 0.0 & 0.0 & 89 & 18.0 & 139 \\
\hline $10: 35$ & $0: 34$ & 75.0 & 40.0 & 1.1 & 89 & 17.0 & 131 \\
\hline $10: 37$ & $0: 36$ & 75.0 & 40.0 & 1.1 & 90 & 18.7 & 144 \\
\hline $10: 39$ & $0: 38$ & 74.0 & 40.0 & 1.1 & 90 & 18.8 & 145 \\
\hline $10: 41$ & $0: 40$ & 75.0 & 40.0 & 1.1 & 90 & 18.7 & 144 \\
\hline $10: 43$ & $0: 42$ & 75.0 & 40.0 & 1.1 & 89 & 18.7 & 144 \\
\hline $10: 45$ & $0: 44$ & 75.0 & 40.0 & 1.1 & 89 & 17.6 & 136 \\
\hline $10: 47$ & $0: 46$ & 75.0 & 41.0 & 1.1 & 90 & 18.8 & 145 \\
\hline $10: 49$ & $0: 48$ & 75.0 & 40.0 & 1.1 & 90 & 18.0 & 139 \\
\hline $10: 51$ & $0: 50$ & 75.0 & 41.0 & 1.1 & 89 & 18.4 & 142 \\
\hline $10: 53$ & $0: 52$ & 0.0 & 0.0 & 0.0 & 89 & 18.4 & 142 \\
\hline $10: 55$ & $0: 54$ & 75.0 & 41.0 & 1.1 & 89 & 1.5 & 12 \\
\hline $10: 57$ & $0: 56$ & 75.0 & 40.0 & 1.1 & 90 & 18.7 & 144 \\
\hline $11: 00$ & $0: 58$ & 75.0 & 41.0 & 1.1 & 89 & 18.4 & 142 \\
\hline $11: 02$ & $1: 00$ & 75.0 & 40.0 & 1.1 & 90 & 18.1 & 140 \\
\hline $11: 04$ & $1: 02$ & 75.0 & 41.0 & 1.1 & 89 & 18.0 & 139 \\
\hline $11: 06$ & $1: 04$ & 75.0 & 40.0 & 1.1 & 90 & 17.6 & 136 \\
\hline $11: 08$ & $1: 06$ & 75.0 & 40.0 & 1.1 & 89 & 17.6 & 136 \\
\hline $11: 10$ & $1: 08$ & 0.0 & 0.0 & 0.0 & 89 & 17.9 & 138 \\
\hline $11: 12$ & $1: 10$ & 75.0 & 40.0 & 1.1 & 88 & 17.9 & 138 \\
\hline $11: 14$ & $1: 12$ & 75.0 & 41.0 & 1.1 & 90 & 16.8 & 130 \\
\hline $11: 16$ & $1: 14$ & 75.0 & 40.0 & 1.1 & 89 & 18.5 & 143 \\
\hline $11: 18$ & $1: 16$ & 75.0 & 41.0 & 1.1 & 90 & 18.2 & 140 \\
\hline $11: 20$ & $1: 18$ & 75.0 & 40.0 & 1.1 & 90 & 18.1 & 140 \\
\hline $11: 22$ & $1: 20$ & 75.0 & 40.0 & 1.1 & 90 & 18.0 & 139 \\
\hline $11: 24$ & $1: 22$ & 75.0 & 41.0 & 1.1 & 89 & 17.8 & 137 \\
\hline $11: 26$ & $1: 24$ & 75.0 & 40.0 & 1.1 & 90 & 17.9 & 138 \\
\hline $11: 57$ & $=1: 56$ & 75.0 & 40.0 & 1.1 & 90 & 17.9 & 138 \\
\hline
\end{tabular}


SCRN4.XLS

\begin{tabular}{|c|c|c|c|c|c|c|c|}
\hline \multicolumn{8}{|c|}{0.3 um $\mathrm{ZrO}_{2} / \mathrm{TiO}_{2}$} \\
\hline \multirow[t]{2}{*}{$\begin{array}{l}\text { TIME OF } \\
\text { DAY }\end{array}$} & $\begin{array}{c}\text { ELAPSED } \\
\text { TIME }\end{array}$ & $\begin{array}{c}\text { FEED } \\
\text { PRESSURE }\end{array}$ & $\begin{array}{c}\text { CONC } \\
\text { PRESSURE }\end{array}$ & $\begin{array}{c}\text { FEED FLOW } \\
\text { (Umin) }\end{array}$ & FEED TEMP & $\begin{array}{l}\text { PERMEATE } \\
\text { FLOW }\end{array}$ & $\begin{array}{l}\text { PERMEATE } \\
\text { FLUX }\end{array}$ \\
\hline & (HH:MM) & (psig) & (psig) & (gpm) & $\left({ }^{\circ} \mathrm{F}\right)$ & $(\mathrm{m} / \mathrm{min})$ & (gal/f/t -day) \\
\hline $11: 58$ & $1: 56$ & 75.0 & 40.0 & 1.1 & 90 & 17.9 & 138 \\
\hline $12: 00$ & $1: 58$ & 75.0 & 40.0 & 1.1 & 90 & 17.9 & 138 \\
\hline $12: 02$ & $2: 01$ & 75.0 & 40.0 & 1.1 & 90 & 17.9 & 138 \\
\hline $12: 05$ & $2: 03$ & 75.0 & 40.0 & 1.1 & 90 & 17.9 & 138 \\
\hline 12:06 & $2: 04$ & 75.0 & 40.0 & 1.1 & 90 & 17.9 & 138 \\
\hline $12: 09$ & $2: 08$ & 75.0 & 40.0 & 1.1 & 90 & 17.9 & 138 \\
\hline $12: 11$ & $2: 09$ & 75.0 & 40.0 & 1.1 & 90 & 17.9 & 138 \\
\hline $12: 15$ & $2: 14$ & 75.0 & 40.0 & 1.1 & 90 & 17.9 & 138 \\
\hline $12: 19$ & $2: 17$ & 75.0 & 40.0 & 1.1 & 90 & 17.9 & 138 \\
\hline Average & & 74.9 & 40.2 & 1.1 & 89.6 & 17.7 & 136.9 \\
\hline & & & & & & & \\
\hline \multicolumn{4}{|c|}{ Average Test Conditions: SCRN4.XLS } & \multicolumn{4}{|c|}{$\frac{1}{1} \frac{1}{1}=72.9 \mathrm{psig}$} \\
\hline & & & & \multicolumn{4}{|c|}{ Concentrate Pressure $=39.1$ psig } \\
\hline \multicolumn{2}{|c|}{ Date Run: 21-Apr-96 } & & & \multicolumn{3}{|c|}{ Feed Flow $=1.0 \mathrm{gpm}$} & \\
\hline & & & & & Temp. & $=90 \mathrm{~F}$ & \\
\hline
\end{tabular}




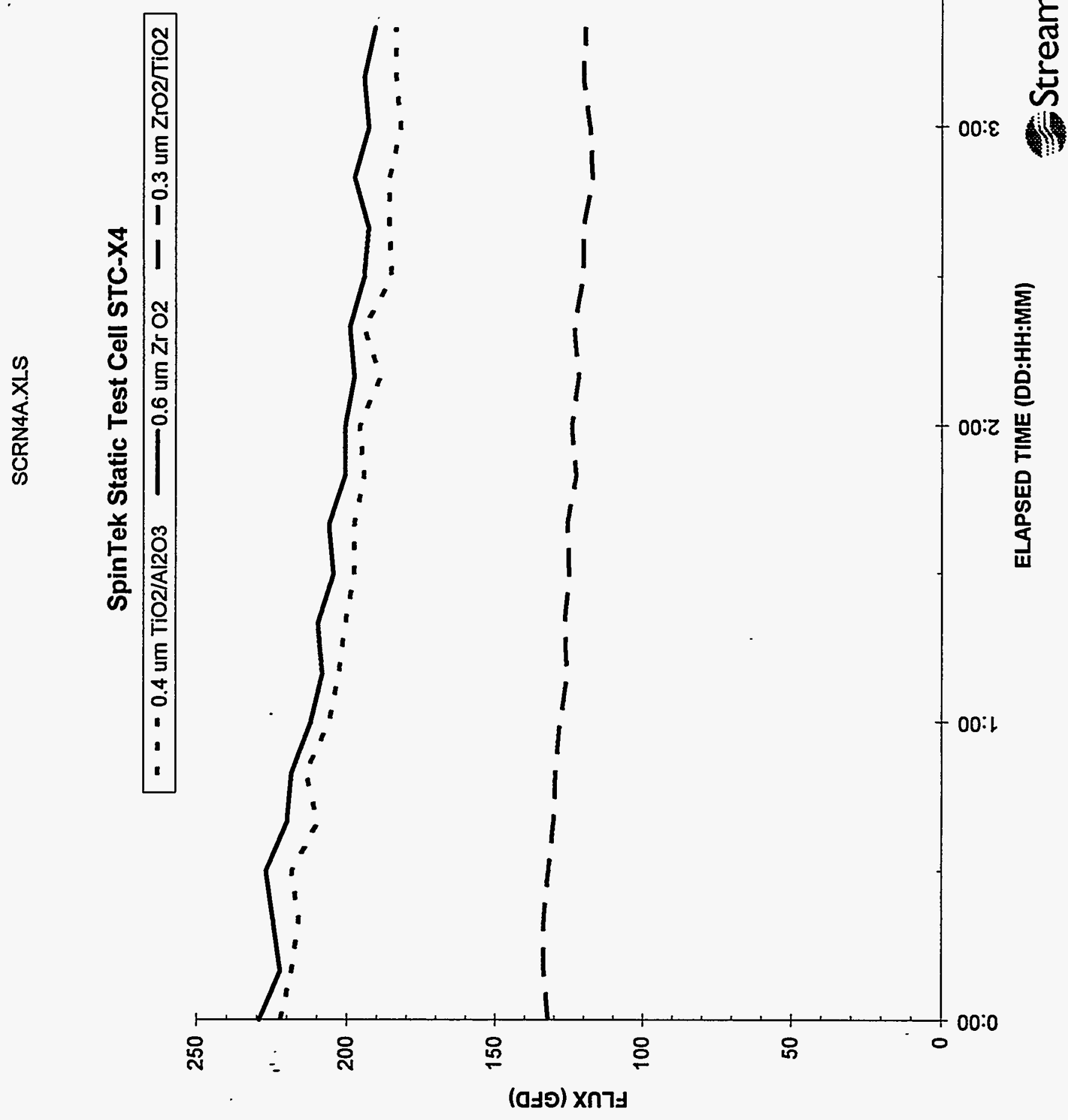




\begin{tabular}{|c|c|c|c|c|c|c|c|}
\hline \multicolumn{8}{|l|}{ SCRN4A } \\
\hline \multicolumn{8}{|c|}{0.4 um $\mathrm{TiO}_{2} / \mathrm{Al}_{2} \mathrm{O}_{3}$} \\
\hline \multirow[t]{2}{*}{ TIME OF DAY } & $\begin{array}{l}\text { ELAPSED } \\
\text { TIME }\end{array}$ & $\begin{array}{c}\text { FEED } \\
\text { PRESSURE }\end{array}$ & $\begin{array}{c}\text { CONC } \\
\text { PRESSURE }\end{array}$ & $\begin{array}{c}\text { FEED FLOW } \\
\text { (L/min) }\end{array}$ & FEED TEMP & $\begin{array}{l}\text { PERMEATE } \\
\text { FLOW }\end{array}$ & $\begin{array}{l}\text { PERMEATE } \\
\text { FLUX }\end{array}$ \\
\hline & (HН:MM) & (psig) & (psig) & (gpm) & $\left({ }^{\circ} \mathrm{F}\right)$ & (mi/min) & (gal/ft' -day) \\
\hline $12: 26$ & $0: 00$. & 75.0 & 40.0 & 1.1 & 90 & 28.8 & 222 \\
\hline $12: 36$ & 0:09 & 0.0 & 0.0 & 0.0 & 89 & 28.3 & 218 \\
\hline $12: 46$ & $0: 19$ & 75.0 & 40.0 & 1.1 & 89 & 28.0 & 216 \\
\hline $12: 56$ & $0: 29$ & 75.0 & 40.0 & 1.1 & 90 & 28.3 & 218 \\
\hline $13: 06$ & $0: 39$ & 75.0 & 40.0 & 1.1 & 90 & 27.2 & 210 \\
\hline $13: 16$ & $0: 49$ & 75.0 & 40.0 & 1.1 & 90 & 27.7 & 214 \\
\hline $13: 26$ & $0: 59$ & 75.0 & 41.0 & 1.1 & 90 & 26.7 & 206 \\
\hline $13: 36$ & $1: 09$ & 39.0 & 21.0 & 0.1 & 89 & 26.3 & 203 \\
\hline $13: 46$ & $1: 19$ & 75.0 & 40.0 & 1.1 & 90 & 26.0 & 201 \\
\hline $13: 56$ & $1: 29$ & 6.0 & 3.0 & 0.7 & 90 & 25.6 & 198 \\
\hline $14: 06$ & $1: 39$ & 75.0 & 41.0 & 1.1 & 89 & 25.6 & 198 \\
\hline $14: 16$ & $1: 49$ & 75.0 & 40.0 & 1.1 & 90 & 25.2 & 194 \\
\hline $14: 26$ & $1: 59$ & 75.0 & 41.0 & 1.1 & 89 & 25.4 & 196 \\
\hline $14: 36$ & $2: 09$ & 75.0 & 40.0 & 1.1 & 89 & 24.5 & 189 \\
\hline $14: 46$ & $2: 19$ & 75.0 & 40.0 & 1.1 & 89 & 25.2 & 194 \\
\hline $14: 56$ & $2: 30$ & 0.0 & 0.0 & 0.0 & 89 & 24.0 & 185 \\
\hline $15: 06$ & $2: 39$ & 75.0 & 40.0 & 1.1 & 89 & 24.1 & 186 \\
\hline $15: 16$ & $2: 50$ & 75.0 & 40.0 & 1.1 & 89 & 24.1 & 186 \\
\hline $15: 26$ & $3: 00$ & 0.0 & 0.0 & 0.0 & 89 & 23.6 & 182 \\
\hline $15: 36$ & $3: 10$ & 75.0 & 40.0 & 1.1 & 89 & 23.8 & 184 \\
\hline $15: 46$ & $3: 20$ & 75.0 & 40.0 & 1.1 & 90 & 23.8 & 184 \\
\hline Average & & 72.9 & 39.1 & 1.0 & 89.5 & 25.9 & 200.1 \\
\hline & & & & & & & \\
\hline \multicolumn{4}{|c|}{ Average Test Conditions: SCRN4A.XLS } & \multicolumn{4}{|c|}{ Feed Pressure $=72.9 \mathrm{psig}$} \\
\hline & & & & \multicolumn{4}{|c|}{ Concentrate Pressure $=39.1 \mathrm{psig}$} \\
\hline \multicolumn{2}{|c|}{ Date Run: 21-Apr-96 } & & & \multicolumn{3}{|c|}{ Feed Flow $=1.0 \mathrm{gpm}$} & \\
\hline & & & & & Temp. & $=90 \mathrm{~F}$ & \\
\hline
\end{tabular}




\begin{tabular}{|c|c|c|c|c|c|c|c|}
\hline SCRN4A & & & & & & & \\
\hline $0.6 \mathrm{um}$ & $\mathrm{Zr} \mathrm{O}$ & & & & & & \\
\hline $\begin{array}{c}\text { TIME OF } \\
\text { DAY }\end{array}$ & $\begin{array}{l}\text { ELAPSED } \\
\text { TIME }\end{array}$ & $\begin{array}{c}\text { FEED } \\
\text { PRESSURE }\end{array}$ & $\begin{array}{c}\text { CONC } \\
\text { PRESSURE }\end{array}$ & $\begin{array}{c}\text { FEED FLOW } \\
\text { (L/min) }\end{array}$ & FEED TEMP & $\begin{array}{c}\text { PERMEATE } \\
\text { FLOW }\end{array}$ & $\begin{array}{l}\text { PERMEATE } \\
\text { FLUX }\end{array}$ \\
\hline & (HH:MM) & (psig) & (psig) & (gpm) & $\left({ }^{\circ} \mathrm{F}\right)$ & $(\mathrm{m} / \mathrm{min})$ & (gal/fitaday) \\
\hline $12: 26$ & $0: 00$ & 75.0 & 40.0 & 1.1 & 90 & 29.7 & 229 \\
\hline $12: 36$ & $0: 09$ & 0.0 & 0.0 & 0.0 & 89 & 28.8 & 222 \\
\hline $12: 46$ & $0: 19$ & 75.0 & 40.0 & 1.1 & 89 & 29.1 & 225 \\
\hline $12: 56$ & $0: 29$ & 75.0 & 40.0 & 1.1 & 90 & 29.4 & 227 \\
\hline $13: 06$ & $0: 39$ & 75.0 & 40.0 & 1.1 & 90 & 28.5 & 220 \\
\hline $13: 16$ & $0: 49$ & 75.0 & 40.0 & 1.1 & 90 & 28.3 & 218 \\
\hline $13: 26$ & $0: 59$ & 75.0 & 41.0 & 1.1 & 90 & 27.5 & 212 \\
\hline $13: 36$ & $1: 09$ & 39.0 & 21.0 & 0.1 & 89 & 27.0 & 208 \\
\hline $13: 46$ & $1: 19$ & 75.0 & 40.0 & 1.1 & 90 & 27.2 & 210 \\
\hline $13: 56$ & $1: 29$ & 6.0 & 3.0 & 0.7 & 90 & 26.5 & 205 \\
\hline $14: 06$ & $1: 39$ & 75.0 & 41.0 & 1.1 & 89 & 26.7 & 206 \\
\hline $14: 16$ & $1: 49$ & 75.0 & 40.0 & 1.1 & 90 & 26.0 & 201 \\
\hline $14: 26$ & $1: 59$ & 75.0 & 41.0 & 1.1 & 89 & 26.0 & 201 \\
\hline $14: 36$ & $2: 09$ & 75.0 & 40.0 & 1.1 & 89 & 25.6 & 198 \\
\hline $14: 46$ & $2: 19$ & 75.0 & 40.0 & 1.1 & 89 & 25.8 & 199 \\
\hline $14: 56$ & $2: 29$ & 0.0 & 0.0 & 0.0 & 89 & 25.2 & 194 \\
\hline $15: 06$ & $2: 39$ & 75.0 & 40.0 & 1.1 & 89 & 25.0 & 193 \\
\hline $15: 16$ & $2: 49$ & 75.0 & 40.0 & 1.1 & 89 & 25.6 & 198 \\
\hline $15: 26$ & $3: 00$ & 0.0 & 0.0 & 0.0 & 89 & 25.0 & 193 \\
\hline $15: 36$ & $3: 10$ & 75.0 & 40.0 & 1.1 & 89 & 25.2 & 194 \\
\hline $15: 46$ & $3: 20$ & 75.0 & 40.0 & 1.1 & 90 & 24.7 & 191 \\
\hline Average & & 72.9 & 39.1 & 1.0 & 89.5 & 26.9 & 207.6 \\
\hline Average T & st Conditio & ns: SCRN4 & A.XLS & $\mathrm{Fee}$ & d Pressure & $=72.9 \mathrm{psig}$ & \\
\hline & & & & Concentrat & e Pressure & $=39.1$ psig & \\
\hline Date Run: & 21-Apr-96 & & & & Feed Flow & $=1.0 \mathrm{gpm}$ & \\
\hline & & & & & Temp. & $=90 \mathrm{~F}$ & \\
\hline
\end{tabular}




\begin{tabular}{|c|c|c|c|c|c|c|c|}
\hline SCRN4A & & & & & & & \\
\hline $0.3 \mathrm{um}$ & $\mathrm{ZrO}_{2} / \mathrm{Ti}$ & & & & & & \\
\hline $\begin{array}{c}\text { TIME OF } \\
\text { DAY }\end{array}$ & $\begin{array}{c}\text { ELAPSED } \\
\text { TIME }\end{array}$ & $\begin{array}{c}\text { FEED } \\
\text { PRESSURE }\end{array}$ & $\begin{array}{c}\text { CONC } \\
\text { PRESSURE }\end{array}$ & $\begin{array}{c}\text { FEED FLOW } \\
\text { (L/min) }\end{array}$ & FEED TEMP & $\begin{array}{c}\text { PERMEATE } \\
\text { FLOW }\end{array}$ & $\begin{array}{l}\text { PERMEATE } \\
\text { FLUX }\end{array}$ \\
\hline & (HH:MM) & (psig) & (psig) & (gpm) & $\left({ }^{\circ} \mathrm{F}\right)$ & $(\mathrm{m} / \mathrm{min})$ & (gal/fit ${ }^{2}$-day) \\
\hline $12: 26$ & $0: 00$ & 75.0 & 40.0 & 1.1 & 90 & 17.1 & 132 \\
\hline $12: 36$ & $0: 09$ & 0.0 & 0.0 & 0.0 & 89 & 17.3 & 134 \\
\hline $12: 46$ & $0: 19$ & 75.0 & 40.0 & 1.1 & 89 & 17.3 & 134 \\
\hline $12: 56$ & $0: 29$ & 75.0 & 40.0 & 1.1 & 90 & 17.1 & 132 \\
\hline $13: 06$ & $0: 39$ & 75.0 & 40.0 & 1.1 & 90 & 16.9 & 130 \\
\hline $13: 16$ & $0: 49$ & 75.0 & 40.0 & 1.1 & 90 & 16.8 & 130 \\
\hline $13: 26$ & $0: 59$ & 75.0 & 41.0 & 1.1 & 90 & 16.6 & 128 \\
\hline $13: 36$ & $1: 09$ & 39.0 & 21.0 & 0.1 & 89 & 16.3 & 126 \\
\hline $13: 46$ & $1: 19$ & 75.0 & 40.0 & 1.1 & 90 & 16.4 & 127 \\
\hline $13: 56$ & $1: 29$ & 6.0 & 3.0 & 0.7 & 90 & 16.2 & 125 \\
\hline $14: 06$ & $1: 39$ & 75.0 & 41.0 & 1.1 & 89 & 16.3 & 126 \\
\hline $14: 16$ & $1: 49$ & 75.0 & 40.0 & 1.1 & 90 & 15.9 & 123 \\
\hline $14: 26$ & $1: 59$ & 75.0 & 41.0 & 1.1 & 89 & 16.1 & 124 \\
\hline $14: 36$ & $2: 09$ & 75.0 & 40.0 & 1.1 & 89 & 15.8 & 122 \\
\hline $14: 46$ & $2: 19$ & 75.0 & 40.0 & 1.1 & 89 & 16.0 & 123 \\
\hline $14: 56$ & $2: 29$ & 0.0 & 0.0 & 0.0 & 89 & 15.6 & 120 \\
\hline $15: 06$ & $2: 39$ & 75.0 & 40.0 & 1.1 & 89 & 15.6 & 120 \\
\hline $15: 16$ & $2: 49$ & 75.0 & 40.0 & 1.1 & 89 & 15.2 & 117 \\
\hline $15: 26$ & $3: 00$ & 0.0 & 0.0 & 0.0 & 89 & 15.3 & 118 \\
\hline $15: 36$ & $3: 10$ & 75.0 & 40.0 & 1.1 & 89 & 15.6 & 120 \\
\hline $15: 46$ & $3: 20$ & 75.0 & 40.0 & 1.1 & 90 & 15.5 & 120 \\
\hline Average & & 72.9 & 39.1 & 1.0 & 89.5 & 16.3 & 125.5 \\
\hline \begin{tabular}{|l} 
Average Te \\
\end{tabular} & st Conditic & ns: SCRNA & $A X \mid S$ & F & Pressure & 729 nsia & \\
\hline & & & & Concentrat & e Pressure & $=39.1 \mathrm{psig}$ & \\
\hline Date Run: & $1-A p r-96$ & & & & Feed Flow & $=1.0 \mathrm{gpm}$ & \\
\hline & & & & & Temp. & $=90 \mathrm{~F}$ & \\
\hline
\end{tabular}




\section{SpinTek Static Test Cell STC-X4}

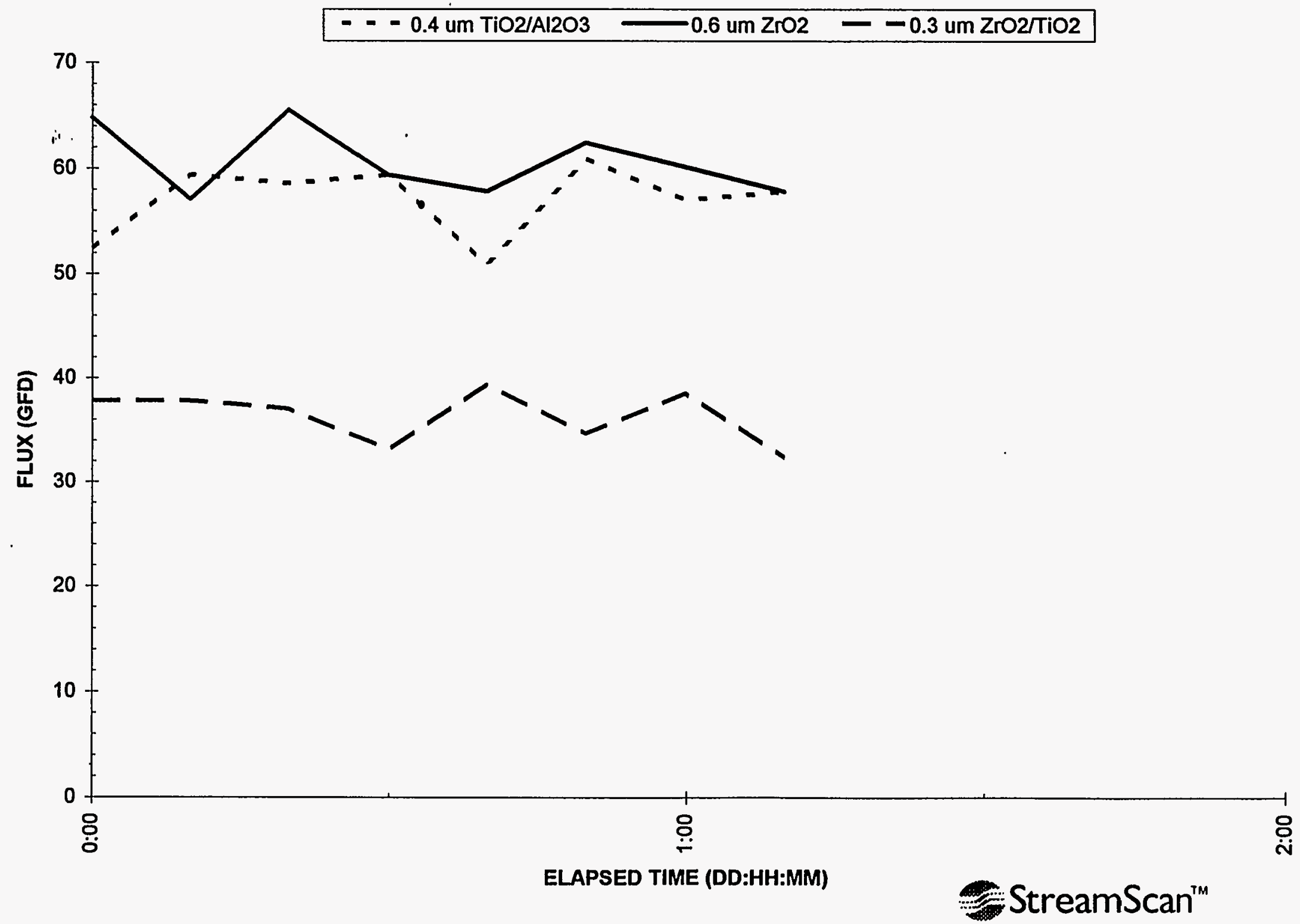


SCRN5.XLS

\begin{tabular}{|c|c|c|c|c|c|c|c|}
\hline \multicolumn{8}{|l|}{ SCRN5 } \\
\hline \multicolumn{8}{|c|}{$0.4 \mathrm{um} \mathrm{TiO} / \mathrm{Al}_{2} \mathrm{O}_{3}$} \\
\hline \multirow[t]{2}{*}{$\begin{array}{c}\text { TIME OF } \\
\text { DAY }\end{array}$} & $\begin{array}{l}\text { ELAPSED } \\
\text { TIME }\end{array}$ & $\begin{array}{c}\text { FEED } \\
\text { PRESSURE }\end{array}$ & $\begin{array}{c}\text { CONC } \\
\text { PRESSURE }\end{array}$ & $\begin{array}{c}\text { FEED FLOW } \\
\text { (L/min) }\end{array}$ & FEED TEMP & $\begin{array}{l}\text { PERMEATE } \\
\text { FLOW }\end{array}$ & $\begin{array}{l}\text { PERMEATE } \\
\text { FLUX }\end{array}$ \\
\hline & (HH:MM) & (psig) & (psig) & (gpm) & $\left({ }^{\circ} \mathrm{F}\right)$ & (m/min) & (gal/ft' $-d a y)$ \\
\hline $16: 22$ & $0: 00$ & 29.0 & 6.0 & 0.9 & 89 & 6.8 & 52 \\
\hline $16: 32$ & $0: 09$ & 27.0 & 5.0 & 0.8 & 89 & 7.7 & $\overline{59}$ \\
\hline $16: 42$ & $0: 19$ & 28.0 & 5.0 & 0.8 & 90 & 7.6 & 59 \\
\hline $16: 52$ & $0: 29$ & 29.0 & 6.0 & 0.9 & 90 & 7.7 & 59 \\
\hline $17: 02$ & $0: 39$ & 28.0 & 6.0 & 0.9 & 89 & 6.6 & 51 \\
\hline $17: 12$ & $0: 49$ & 30.0 & 6.0 & 0.9 & 90 & 7.9 & 61 \\
\hline $17: 22$ & $0: 59$ & 29.0 & 6.0 & 0.9 & 90 & 7.4 & 57 \\
\hline $17: 32$ & $1: 09$ & 28.0 & 5.0 & 0.9 & 90 & 7.5 & 58 \\
\hline Average & & 28.5 & 5.6 & 0.9 & 89.6 & 7.4 & 57.1 \\
\hline \multicolumn{4}{|c|}{ Average Test Conditions: SCRN5.XLS } & \multicolumn{4}{|c|}{ Feed Pressure $=28.5 \mathrm{psig}$} \\
\hline & & & & Concentrat & Pressure & $=5.6 \mathrm{psig}$ & \\
\hline \multirow{2}{*}{\multicolumn{2}{|c|}{ Date Run: 21-Apr-96 }} & & & & \multicolumn{2}{|c|}{ Feed Flow $=0.9 \mathrm{gpm}$} & \\
\hline & & & & & \multicolumn{2}{|c|}{ Temp. $=90 \mathrm{~F}$} & \\
\hline
\end{tabular}


SCRN5.XLS

\begin{tabular}{|c|c|c|c|c|c|c|c|}
\hline SCRN5 & & & & & & & \\
\hline $0.6 \mathrm{um}$ & $\mathrm{ZrO}_{2}$ & & & & & & \\
\hline $\begin{array}{c}\text { TIME OF } \\
\text { DAY }\end{array}$ & $\begin{array}{l}\text { ELAPSED } \\
\text { TIME }\end{array}$ & $\begin{array}{c}\text { FEED } \\
\text { PRESSURE }\end{array}$ & $\begin{array}{c}\text { CONC } \\
\text { PRESSURE }\end{array}$ & $\begin{array}{c}\text { FEED FLOW } \\
\text { (L/min) }\end{array}$ & FEED TEMP & $\begin{array}{l}\text { PERMEATE } \\
\text { FLOW }\end{array}$ & $\begin{array}{l}\text { PERMEATE } \\
\text { FLUX }\end{array}$ \\
\hline & (HH:MM) & (psig) & (psig) & (gpm) & $\left({ }^{\circ} \mathrm{F}\right)$ & $(\mathrm{ml} / \mathrm{min})$ & (gal/ft'tay) \\
\hline $16: 22$ & $0: 00$ & 29.0 & 6.0 & 0.9 & 89 & 8.4 & 65 \\
\hline $16: 32$ & $0: 09$ & 27.0 & 5.0 & 0.8 & 89 & 7.4 & 57 \\
\hline $16: 42$ & $0: 19$ & 28.0 & 5.0 & 0.8 & 90 & 8.5 & 66 \\
\hline $16: 52$ & $0: 29$ & 29.0 & 6.0 & 0.9 & 90 & 7.7 & 59 \\
\hline $17: 02$ & $0: 39$ & 28.0 & 6.0 & 0.9 & 89 & 7.5 & 58 \\
\hline $17: 12$ & $0: 49$ & 30.0 & 6.0 & 0.9 & 90 & 8.1 & 63 \\
\hline $17: 22$ & $0: 59$ & 29.0 & 6.0 & 0.9 & 90 & 7.8 & 60 \\
\hline $17: 32$ & $1: 09$ & 28.0 & 5.0 & 0.9 & 90 & 7.5 & 58 \\
\hline Average & & 28.5 & 5.6 & 0.9 & 89.6 & 7.9 & 60.7 \\
\hline Average T & st Conditic & ns: SCRN5 & $\overline{X L S}$ & Feec & Pressure & $=28.5 \mathrm{psig}$ & \\
\hline & & & & Concentrate & e Pressure & $=5.6 \mathrm{psig}$ & \\
\hline Date Run: & 1-Apr-96 & & & & Feed Flow & $=0.9 \mathrm{gpm}$ & \\
\hline & & & & & Temp. & $=90 \mathrm{~F}$ & \\
\hline
\end{tabular}


SCRN5.XLS

\begin{tabular}{|c|c|c|c|c|c|c|c|}
\hline SCRN5 & & & & & & & \\
\hline 0.3 um & $\mathrm{ZrO}_{2} / \mathrm{Ti}$ & & & & & & \\
\hline $\begin{array}{c}\text { TIME OF } \\
\text { DAY }\end{array}$ & $\begin{array}{c}\text { ELAPSED } \\
\text { TIME }\end{array}$ & $\begin{array}{c}\text { FEED } \\
\text { PRESSURE }\end{array}$ & $\begin{array}{c}\text { CONC } \\
\text { PRESSURE }\end{array}$ & $\begin{array}{c}\text { FEED FLOW } \\
\text { (L/min) }\end{array}$ & FEED TEMP & $\begin{array}{c}\text { PERMEATE } \\
\text { FLOW }\end{array}$ & $\begin{array}{l}\text { PERMEATE } \\
\text { FLUX }\end{array}$ \\
\hline & (HH:MM) & (psig) & (psig) & (gpm) & $\left({ }^{\circ} \mathrm{F}\right)$ & $(\mathrm{m} / \mathrm{min})$ & (gal/ft ${ }^{2}$-day) \\
\hline $16: 22$ & $0: 00$ & 29.0 & 6.0 & 0.9 & 89 & 4.9 & 38 \\
\hline $16: 32$ & $0: 09$ & 27.0 & 5.0 & 0.8 & 89 & 4.9 & 38 \\
\hline $16: 42$ & $0: 19$ & 28.0 & 5.0 & 0.8 & 90 & 4.8 & 37 \\
\hline $16: 52$ & $0: 29$ & 29.0 & 6.0 & 0.9 & 90 & 4.3 & 33 \\
\hline $17: 02$ & $0: 39$ & 28.0 & 6.0 & 0.9 & 89 & 5.1 & 39 \\
\hline $17: 12$ & $0: 49$ & 30.0 & 6.0 & 0.9 & 90 & 4.5 & 35 \\
\hline $17: 22$ & $0: 59$ & 29.0 & 6.0 & 0.9 & 90 & 5.0 & 39 \\
\hline $17: 32$ & $1: 09$ & 28.0 & 5.0 & 0.9 & 90 & 4.2 & 32 \\
\hline Average & & 28.5 & 5.6 & 0.9 & 89.6 & 4.7 & 36.4 \\
\hline Average T & st Conditi & IS: SCRNE &. $\mathrm{XLS}$ & Fee & Pressure & $=28.5 \mathrm{psig}$ & \\
\hline & & & & Concentrat & te Pressure & $=5.6 \mathrm{psig}$ & \\
\hline Date Run: & 21-Apr-96 & & & & Feed Flow & $=0.9 \mathrm{gpm}$ & \\
\hline & & & & & Temp. & $=90 \mathrm{~F}$ & \\
\hline
\end{tabular}


$\frac{\mathscr{g}}{\stackrel{\circ}{\infty}}$

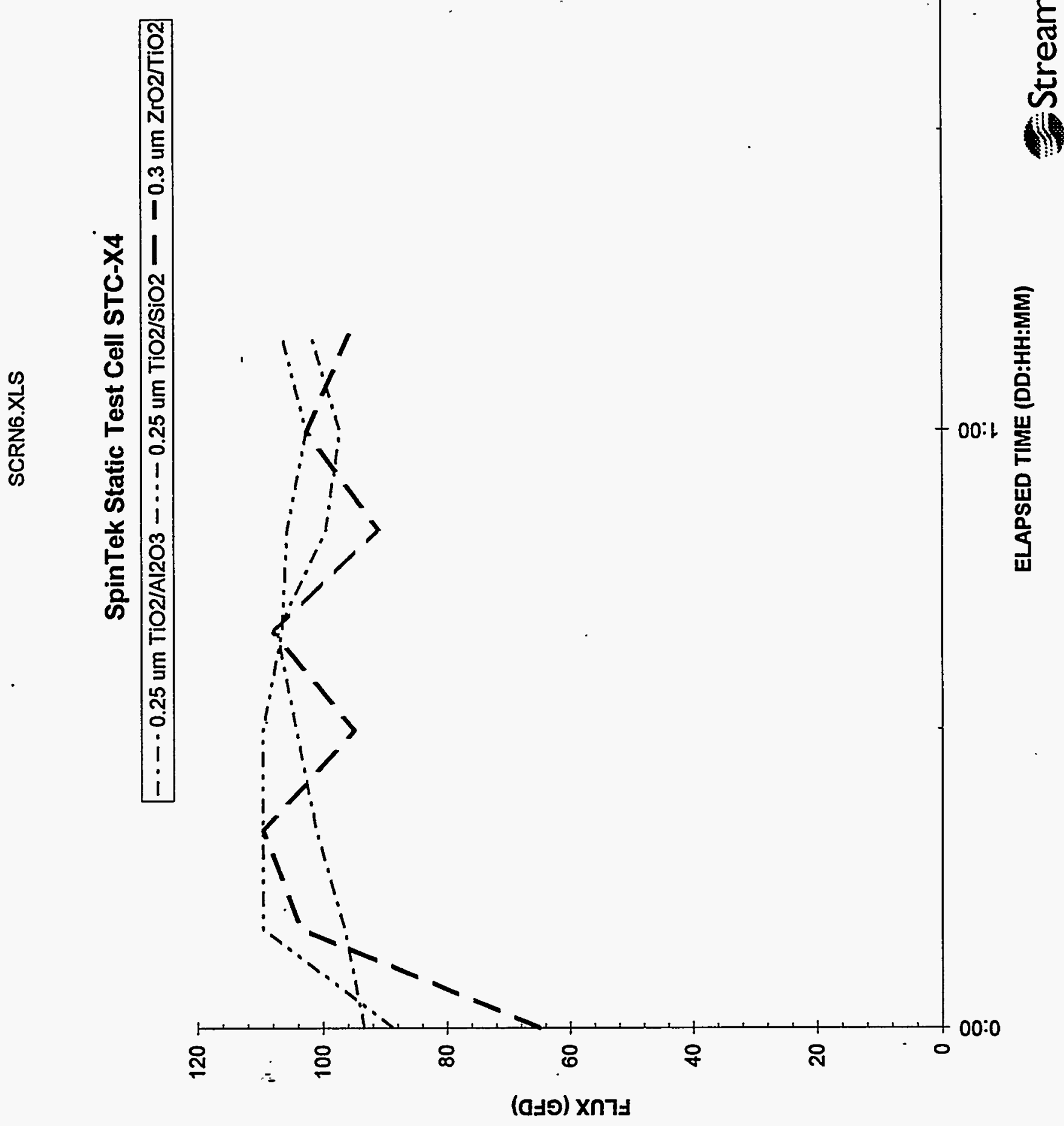




\begin{tabular}{|c|c|c|c|c|c|c|c|}
\hline SCRN6 & & & & & & & \\
\hline 0.25 un & $\mathrm{TiO}_{2} / \mathrm{A}$ & ${ }_{2} \mathrm{O}_{3}$ & & & & & \\
\hline$\underset{\text { DAY }}{\text { TIME OF }}$ & $\begin{array}{l}\text { ELAPSED } \\
\text { TIME }\end{array}$ & $\begin{array}{c}\text { FEED } \\
\text { PRESSURE }\end{array}$ & $\begin{array}{c}\text { CONC } \\
\text { PRESSURE }\end{array}$ & $\begin{array}{c}\text { FEED FLOW } \\
\text { (L/min) }\end{array}$ & FEED TEMP & $\begin{array}{l}\text { PERMEATE } \\
\text { FLOW }\end{array}$ & $\begin{array}{l}\text { PERMEATE } \\
\text { FLUX }\end{array}$ \\
\hline & (HH:MM) & (psig) & (psig) & (gpm) & $\left({ }^{\circ} \mathrm{F}\right)$ & (mU/min) & (gal/fft ${ }^{2}$-day) \\
\hline $18: 10$ & $0: 00$ & 29.0 & 7.0 & 0.9 & 89 & 12.1 & 93 \\
\hline $18: 20$ & $0: 09$ & 30.0 & 7.0 & 0.9 & 89 & 12.5 & 96 \\
\hline $18: 30$ & $0: 19$ & 31.0 & 7.0 & 0.9 & 89 & 13.1 & 101 \\
\hline $18: 40$ & $0: 29$ & 30.0 & 7.0 & 0.9 & 90 & 13.5 & 104 \\
\hline $18: 50$ & $0: 39$ & 31.0 & 7.0 & 0.9 & 89 & 13.9 & 107 \\
\hline $19: 00$ & $0: 49$ & 29.0 & 7.0 & 0.9 & 89 & 12.9 & 100 \\
\hline $19: 10$ & $1: 00$ & 29.0 & 7.0 & 0.9 & 89 & 12.6 & 97 \\
\hline $19: 20$ & $1: 10$ & 30.0 & 7.0 & 0.9 & 90 & 13.2 & 102 \\
\hline Average & & 29.9 & 7.0 & 0.9 & 89.3 & 13.0 & 100.1 \\
\hline & & & & & & & \\
\hline$\overline{\text { Average T }}$ & est Conditic & ns: SCRNG & $\mathrm{XLS}$ & Feed & Pressure $=$ & $=29.9$ psig & \\
\hline & & & & Concentrat & e Pressure & $=7.0 \mathrm{psig}$ & \\
\hline Date Run: & 21-Apr-96 & & & & Feed Flow: & $=0.9 \mathrm{gpm}$ & \\
\hline & & & & & Temp. $=$ & $=89.3 \mathrm{~F}$ & \\
\hline
\end{tabular}


SCRN6.XLS

\begin{tabular}{|c|c|c|c|c|c|c|c|}
\hline SCRN6 & & & & & & & \\
\hline 0.25 ur & $\mathrm{TiO}_{2} / \mathrm{S}$ & $\mathrm{O}_{2}$ & & & & & \\
\hline $\begin{array}{c}\text { TIME OF } \\
\text { DAY }\end{array}$ & $\begin{array}{l}\text { ELAPSED } \\
\text { TIME }\end{array}$ & $\begin{array}{c}\text { FEED } \\
\text { PRESSURE }\end{array}$ & $\begin{array}{c}\text { CONC } \\
\text { PRESSURE }\end{array}$ & $\begin{array}{l}\text { FEED FLOW } \\
\text { (L/min) }\end{array}$ & FEED TEMP & $\begin{array}{l}\text { PERMEATE } \\
\text { FLOW }\end{array}$ & $\begin{array}{l}\text { PERMEATE } \\
\text { FLUX }\end{array}$ \\
\hline & (HH:MM) & (psig) & (psig) & (gpm) & $\left({ }^{\circ} \mathrm{F}\right)$ & $(\mathrm{ml} / \mathrm{min})$ & (gal/ft ${ }^{2}$-day) \\
\hline $18: 10$ & $0: 00$ & 29.0 & 7.0 & 0.9 & 89 & 11.5 & 89 \\
\hline $18: 20$ & $0: 09$ & 30.0 & 7.0 & 0.9 & 89 & 14.2 & 110 \\
\hline $18: 30$ & $0: 19$ & 31.0 & 7.0 & 0.9 & 89 & 14.2 & 110 \\
\hline $18: 40$ & $0: 29$ & 30.0 & 7.0 & 0.9 & 90 & 14.2 & 110 \\
\hline $18: 50$ & $0: 39$ & 31.0 & 7.0 & 0.9 & 89 & 13.8 & 106 \\
\hline $19: 00$ & $0: 49$ & 29.0 & 7.0 & 0.9 & 89 & 13.7 & 106 \\
\hline $19: 10$ & $1: 00$ & 29.0 & 7.0 & 0.9 & 89 & 13.3 & 103 \\
\hline $19: 20$ & $1: 10$ & 30.0 & 7.0 & 0.9 & 90 & 13.8 & 106 \\
\hline Average & & 29.9 & 7.0 & 0.9 & 89.3 & 13.6 & 104.9 \\
\hline Average & & & & & & & \\
\hline Average 1 & onditic & SCRNE & $\mathbf{S}$ & $\begin{array}{r}\text { Feec } \\
\text { Concentrat }\end{array}$ & Pressure & $=29.9$ psig & \\
\hline Date Run: & $1-A p r-96$ & & & Vimo & Feed Flow & $=0.9 \mathrm{gpm}$ & \\
\hline & & & & & Temp. $=$ & $=89.3 \mathrm{~F}$ & \\
\hline
\end{tabular}


SCRN6.XLS

\begin{tabular}{|c|c|c|c|c|c|c|c|}
\hline SCRN6 & & & & & & & \\
\hline $0.3 \mathrm{um}$ & $\mathrm{ZrO}_{2} / \mathrm{TI}$ & & & & & & \\
\hline $\begin{array}{l}\text { TIME OF } \\
\text { DAY }\end{array}$ & $\begin{array}{c}\text { ELAPSED } \\
\text { TIME }\end{array}$ & $\begin{array}{c}\text { FEED } \\
\text { PRESSURE }\end{array}$ & $\begin{array}{c}\text { CONC } \\
\text { PRESSURE }\end{array}$ & $\begin{array}{c}\text { FEED FLOW } \\
\text { (L/min) }\end{array}$ & FEED TEMP & $\begin{array}{c}\text { PERMEATE } \\
\text { FLOW }\end{array}$ & $\begin{array}{l}\text { PERMEATE } \\
\text { FLUX }\end{array}$ \\
\hline & (HH:MM) & (psig) & (psig) & (gpm) & $\left({ }^{\circ} \mathrm{F}\right)$ & (mU/min) & 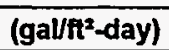 \\
\hline $18: 10$ & $0: 00$ & 29.0 & 7.0 & 0.9 & 89 & 8.4 & 65 \\
\hline $18: 20$ & $0: 09$ & 30.0 & 7.0 & 0.9 & 89 & 13.4 & 103 \\
\hline $18: 30$ & $0: 19$ & 31.0 & 7.0 & 0.9 & 89 & 14.2 & 110 \\
\hline $18: 40$ & $0: 29$ & 30.0 & 7.0 & 0.9 & 90 & 12.3 & 95 \\
\hline $18: 50$ & $0: 39$ & 31.0 & 7.0 & 0.9 & 89 & 14.0 & 108 \\
\hline $19: 00$ & $0: 49$ & 29.0 & 7.0 & 0.9 & 89 & 11.8 & 91 \\
\hline $19: 10$ & $1: 00$ & 29.0 & 7.0 & 0.9 & 89 & 13.3 & 103 \\
\hline $19: 20$ & $1: 10$ & 30.0 & 7.0 & 0.9 & 90 & 12.4 & 96 \\
\hline Average & & 29.9 & 7.0 & 0.9 & 89.3 & 12.5 & 96.3 \\
\hline \begin{tabular}{|l} 
Average T \\
\end{tabular} & t Conditi & s: SCRN6 & XLS & Feec & Pressure & $=29.9 \mathrm{Dsia}$ & \\
\hline & & & & Concentrat & e Pressure & $=7.0 \mathrm{psig}$ & \\
\hline Date Run: & 1-Apr-96 & & & & Feed Flow & $=0.9 \mathrm{gpm}$ & \\
\hline & & & & & Temp. $=$ & $=89.3 \mathrm{~F}$ & \\
\hline
\end{tabular}


ֻั

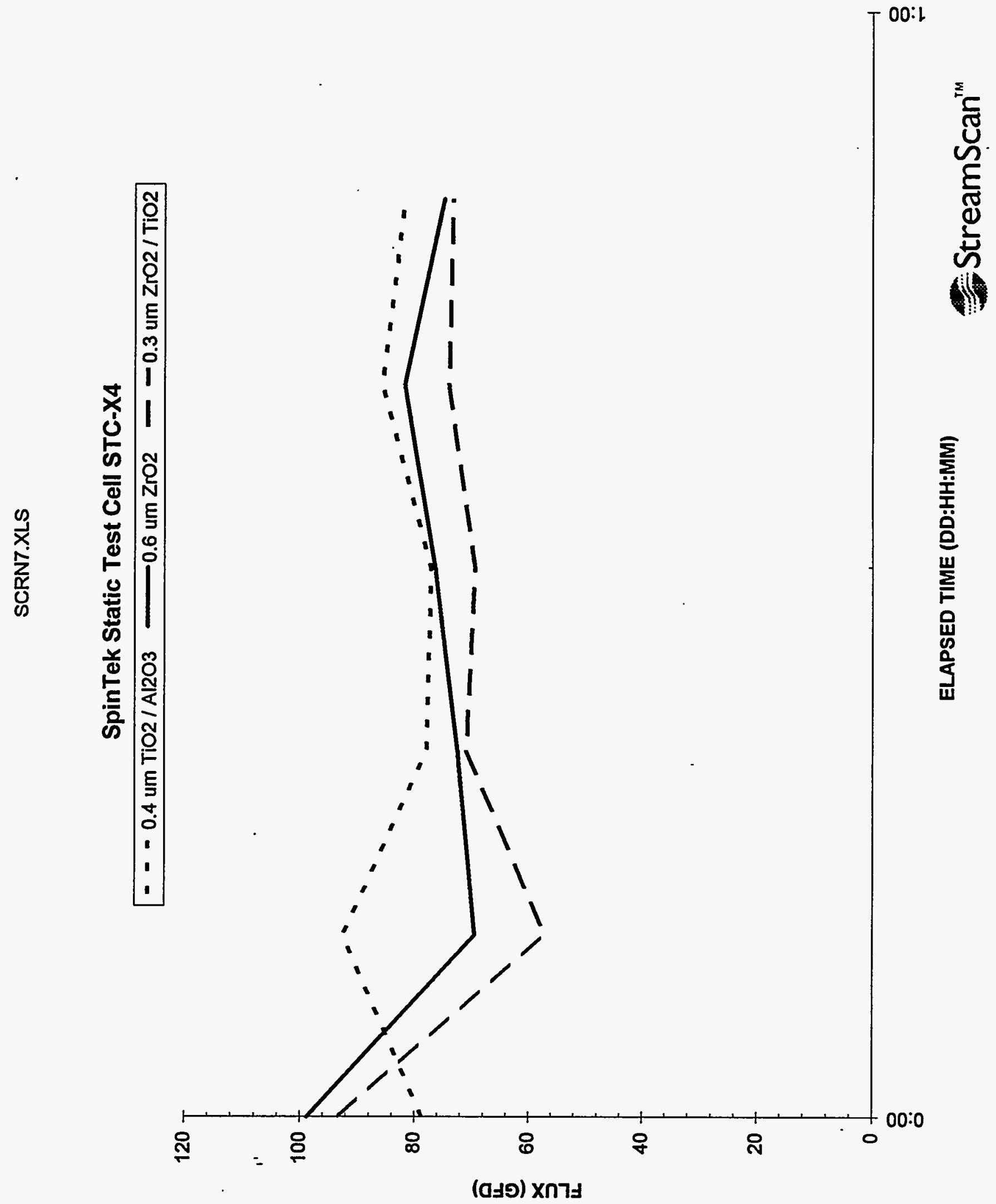




\begin{tabular}{|c|c|c|c|c|c|c|c|}
\hline \multicolumn{8}{|l|}{ SCRN7 } \\
\hline \multicolumn{8}{|c|}{$0.4 \mathrm{um} \mathrm{TiO}_{2} / \mathrm{Al}_{2} \mathrm{O}_{3}$} \\
\hline \multirow[t]{2}{*}{$\begin{array}{c}\text { TIME OF } \\
\text { DAY }\end{array}$} & $\begin{array}{c}\text { ELAPSED } \\
\text { TIME }\end{array}$ & $\begin{array}{c}\text { FEED } \\
\text { PRESSURE }\end{array}$ & $\begin{array}{c}\text { CONC } \\
\text { PRESSURE }\end{array}$ & $\begin{array}{c}\text { FEED FLOW } \\
\text { (L'min) }\end{array}$ & FEED TEMP & $\begin{array}{c}\text { PERMEATE } \\
\text { FLOW }\end{array}$ & $\begin{array}{l}\text { PERMEATE } \\
\text { FLUX }\end{array}$ \\
\hline & (HH:MM) & (psig) & (psig) & (gpm) & $\left({ }^{\circ} \mathrm{F}\right)$ & (mU/min) & (gal/ft ${ }^{2}$-day) \\
\hline $21: 01$ & $0: 00$ & 32.0 & 6.0 & 0.9 & 90 & 10.2 & 79 \\
\hline $21: 11$ & $0: 09$ & 31.0 & 6.0 & 0.9 & 89 & 12.0 & 93 \\
\hline $21: 21$ & $0: 19$ & 28.0 & 6.0 & 0.9 & 90 & 10.1 & 78 \\
\hline $21: 31$ & $0: 29$ & 29.0 & 6.0 & 0.9 & 90 & 10.0 & 77 \\
\hline $21: 41$ & $0: 39$ & 28.0 & 6.0 & 0.9 & 90 & 11.1 & 86 \\
\hline $21: 51$ & $0: 49$ & 27.0 & 6.0 & 0.9 & 90 & 10.6 & 82 \\
\hline Average & & 29.2 & 6.0 & 0.9 & 89.8 & 10.7 & 82.3 \\
\hline & & & & & & & \\
\hline \multicolumn{4}{|c|}{ Average Test Conditions: SCRN7.XLS } & \multicolumn{3}{|c|}{ Feed Pressure $=29.2 \mathrm{psig}$} & \\
\hline & & & & \multicolumn{3}{|c|}{ Concentrate Pressure $=6.0 \mathrm{psig}$} & \\
\hline \multirow{2}{*}{\multicolumn{2}{|c|}{ Date Run: 22-Apr-96 }} & & & \multicolumn{3}{|c|}{$\begin{aligned} \text { Feed Flow }=0.9 \mathrm{gpm} \\
\text { Temn }=898\end{aligned}$} & \\
\hline & & & & \multicolumn{3}{|c|}{ Temp. $=89.8$} & \\
\hline
\end{tabular}


SCRN7.XLS

\begin{tabular}{|c|c|c|c|c|c|c|c|}
\hline \multicolumn{8}{|l|}{ SCRN7 } \\
\hline \multicolumn{8}{|c|}{$0.6 \mathrm{um} \mathrm{ZrO}_{2}$} \\
\hline \multirow[t]{2}{*}{$\begin{array}{l}\text { TIME OF } \\
\text { DAY }\end{array}$} & $\begin{array}{l}\text { ELAPSED } \\
\text { TIME }\end{array}$ & $\begin{array}{c}\text { FEED } \\
\text { PRESSURE }\end{array}$ & $\begin{array}{c}\text { CONC } \\
\text { PRESSURE }\end{array}$ & $\begin{array}{c}\text { FEED FLOW } \\
\text { (Umin) }\end{array}$ & FEED TEMP & $\begin{array}{l}\text { PERMEATE } \\
\text { FLOW }\end{array}$ & $\begin{array}{l}\text { PERMEATE } \\
\text { FLUX }\end{array}$ \\
\hline & (HH:MM) & (psig) & (psig) & (gpm) & $\left({ }^{\circ} \mathrm{F}\right)$ & (ml/min) & (gal/ $/ t^{2}$-day) \\
\hline $21: 01$ & $0: 00$ & 32.0 & 6.0 & 0.9 & 90 & 12.8 & 99 \\
\hline $21: 11$ & $0: 09$ & 31.0 & 6.0 & 0.9 & 89 & 9.0 & 69 \\
\hline $21: 21$ & $0: 19$ & 28.0 & 6.0 & 0.9 & 90 & 9.4 & 73 \\
\hline $21: 31$ & $0: 29$ & 29.0 & 6.0 & 0.9 & 90 & 9.9 & 76 \\
\hline $21: 41$ & $0: 39$ & 28.0 & 6.0 & 0.9 & 90 & 10.6 & 82 \\
\hline $21: 51$ & $0: 49$ & 27.0 & 6.0 & 0.9 & 90 & 9.7 & 75 \\
\hline Average & & 29.2 & 6.0 & 0.9 & 89.8 & 10.2 & 79.0 \\
\hline \multicolumn{4}{|c|}{ Average Test Conditions: SCRN7.XLS } & \multicolumn{3}{|c|}{ Feed Pressure $=29.2$ psig } & \\
\hline & & & & \multicolumn{3}{|c|}{ Concentrate Pressure $=6.0 \mathrm{psig}$} & \\
\hline \multirow{2}{*}{\multicolumn{2}{|c|}{ Date Run: 22-Apr-96 }} & & & \multirow{2}{*}{\multicolumn{3}{|c|}{$\begin{aligned} \text { Feed Flow } & =0.9 \mathrm{gpm} \\
\text { Temp. } & =89.8\end{aligned}$}} & \\
\hline & & & & & & & \\
\hline
\end{tabular}




\begin{tabular}{|c|c|c|c|c|c|c|c|}
\hline SCRN7 & & & & & & & \\
\hline $0.3 \mathrm{um}$ & $\mathrm{ZrO}_{2} / \mathrm{T}$ & $\mathrm{O}_{2}$ & & & & & \\
\hline $\begin{array}{c}\text { TIME OF } \\
\text { DAY }\end{array}$ & $\begin{array}{l}\text { ELAPSED } \\
\text { TIME }\end{array}$ & $\begin{array}{c}\text { FEED } \\
\text { PRESSURE }\end{array}$ & $\begin{array}{c}\text { CONC } \\
\text { PRESSURE }\end{array}$ & $\begin{array}{c}\text { FEED FLOW } \\
\text { (L'min) }\end{array}$ & FEED TEMP & $\begin{array}{l}\text { PERMEATE } \\
\text { FLOW }\end{array}$ & $\begin{array}{l}\text { PERMEATE } \\
\text { FLUX }\end{array}$ \\
\hline & (HH:MM) & (psig) & (psig) & (gpm) & $\left({ }^{\circ} \mathrm{F}\right)$ & $(\mathrm{mL} / \mathrm{min})$ & (galfft²-day) \\
\hline $21: 01$ & $0: 00$ & 32.0 & 6.0 & 0.9 & 90 & 12.1 & 93 \\
\hline $21: 11$ & $0: 09$ & 31.0 & 6.0 & 0.9 & 89 & 7.4 & 57 \\
\hline $21: 21$ & $0: 19$ & 28.0 & 6.0 & 0.9 & 90 & 9.2 & 71 \\
\hline $21: 31$ & $0: 29$ & 29.0 & 6.0 & 0.9 & 90 & 9.0 & 69 \\
\hline $21: 41$ & $0: 39$ & 28.0 & 6.0 & 0.9 & 90 & 9.6 & 74 \\
\hline $21: 51$ & $0: 49$ & 27.0 & 6.0 & 0.9 & 90 & 9.5 & 73 \\
\hline Average & & 29.2 & 6.0 & 0.9 & 89.8 & 9.5 & 73.1 \\
\hline Averaqe T & st Conditic & ns: SCRN7 & $\mathrm{XLS}$ & Feed & Pressure : & $=29.2 \mathrm{psig}$ & \\
\hline & & & & Concentrat & e Pressure & $=6.0$ psig & \\
\hline Date Run: & $2-A p r-96$ & & & & Feed Flow & $=0.9 \mathrm{gpm}$ & \\
\hline & & & & & Temp. & $=89.8$ & \\
\hline
\end{tabular}


Energy $\mathcal{E}$

Environmental

Research

Center

APPENDIX C

PERFORMANCE EVALUATION TEST DATA 


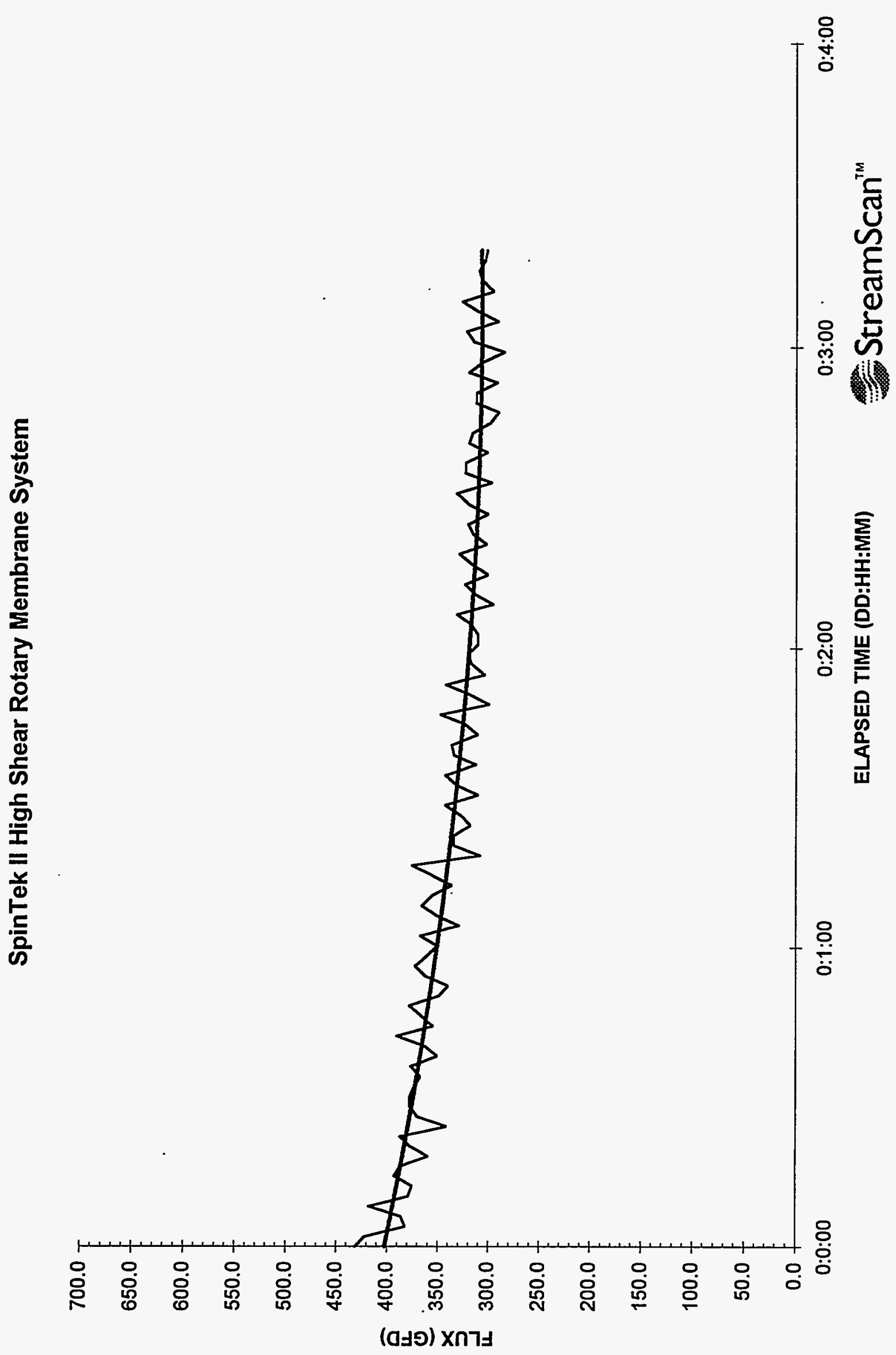




\begin{tabular}{|c|c|c|c|c|c|c|c|c|}
\hline $\begin{array}{c}\text { TIME OF } \\
\text { DAY }\end{array}$ & $\begin{array}{c}\text { ELAPSED } \\
\text { TIME }\end{array}$ & \begin{tabular}{|c|} 
FEED \\
PRESSURE
\end{tabular} & FEED TEMP & FEED FLOW & $\begin{array}{l}\text { ROTOR } \\
\text { SPEED }\end{array}$ & $\begin{array}{c}\text { PERMEATE } \\
\text { FLOW }\end{array}$ & $\begin{array}{c}\text { PERMEATE } \\
\text { FLUX }\end{array}$ & \\
\hline $17: 21$ & $0: 00$ & 44 & 88 & 527 & 1995 & 601 & 431.4 & \\
\hline $17: 23$ & $0: 02$ & 44 & 91 & 543 & 2004 & 588 & 422.1 & \\
\hline $17: 25$ & $0: 04$ & 50 & 91 & 572 & 2006 & 532 & 381.9 & \\
\hline $17: 27$ & $0: 06$ & 45 & 89 & 511 & 1990 & 538 & 386.2 & \\
\hline $17: 29$ & $0: 08$ & 49 & 90 & 539 & 1997 & 582 & 417.8 & \\
\hline $17: 31$ & $0: 10$ & 43 & 92 & 534 & 1995 & 528 & 379.0 & \\
\hline $17: 34$ & $0: 12$ & 43 & 90 & 524 & 1995 & 523 & 375.4 & \\
\hline $17: 36$ & $0: 14$ & 42 & 89 & 533 & 1990 & 547 & 392.7 & \\
\hline $17: 38$ & $0: 16$ & 49 & 91 & 538 & 2003 & 539 & 386.9 & \\
\hline $17: 40$ & $0: 18$ & 44 & 91 & 472 & 1999 & 501 & 359.6 & \\
\hline $17: 42$ & $0: 20$ & 49 & 90 & 475 & 2007 & 526 & 377.6 & \\
\hline $17: 44$ & $0: 22$ & 46 & 91 & 531 & 1991 & 539 & 386.9 & \\
\hline $17: 46$ & $0: 24$ & 49 & 91 & 494 & 2000 & 476 & 341.7 & \\
\hline $17: 48$ & $0: 26$ & 43 & 89 & 527 & 1998 & 516 & 370.4 & \\
\hline $17: 50$ & $0: 28$ & 46 & 90 & 514 & 1999 & 526 & 377.6 & \\
\hline $17: 52$ & $0: 30$ & 46 & 89 & 505 & 1995 & 526 & 377.6 & \\
\hline $17: 54$ & $0: 32$ & 47 & 91 & 514 & 1996 & 520 & 373.3 & \\
\hline $17: 56$ & $0: 34$ & 46 & 89 & 487 & 1991 & 512 & 367.5 & \\
\hline $17: 58$ & $0: 36$ & 47 & 89 & 511 & 1989 & 525 & 376.9 & \\
\hline $18: 00$ & $0: 38$ & 45 & 91 & 514 & 1989 & 489 & 351.0 & \\
\hline 18:02 & $0: 40$ & 47 & 89 & 502 & 1997 & 505 & 362.5 & \\
\hline $18: 04$ & $0: 42$ & 42 & 90 & 509 & 1996 & 544 & 390.5 & \\
\hline $18: 06$ & $0: 44$ & 44 & 90 & 537 & 1991 & 495 & 355.3 & \\
\hline $18: 08$ & $0: 46$ & 45 & 89 & 493 & 1996 & 511 & 366.8 & \\
\hline $18: 10$ & $0: 48$ & 46 & 89 & 545 & 1988 & 527 & 378.3 & \\
\hline $18: 12$ & $0: 50$ & 47 & 92 & 523 & 1991 & 487 & 349.6 & \\
\hline $18: 14$ & $0: 52$ & 48 & 90 & 515 & 1997 & 474 & 340.3 & \\
\hline $18: 16$ & $0: 54$ & 52 & 89 & 483 & 1987 & 505 & 362.5 & \\
\hline $18: 18$ & $0: 56$ & 50 & 90 & 509 & 1989 & 519 & 372.6 & \\
\hline $18: 20$ & $0: 58$ & 45 & 91 & 506 & 2003 & 504 & 361.8 & \\
\hline $18: 22$ & $1: 00$ & 51 & 89 & 507 & 2003 & 489 & 351.0 & \\
\hline $18: 24$ & $1: 02$ & 43 & 89 & 507 & 1990 & 512 & 367.5 & \\
\hline $18: 26$ & $1: 04$ & 43 & 91 & 513 & 2003 & 459 & 329.5 & \\
\hline $18: 28$ & $1: 06$ & 46 & 89 & 524 & 1995 & 489 & 351.0 & \\
\hline $18: 30$ & $1: 08$ & 49 & 89 & 521 & 1991 & 510 & 366.1 & \\
\hline $18: 32$ & $1: 10$ & 46 & 91 & 523 & 1996 & 496 & 356.0 & \\
\hline $18: 34$ & $1: 12$ & 48 & 90 & 487 & 1987 & 469 & 336.7 & \\
\hline $18: 36$ & $1: 14$ & 43 & 89 & 514 & 1997 & 496 & 356.0 & \\
\hline $18: 38$ & $1: 16$ & 49 & 90 & 514 & 1996 & 523 & 375.4 & \\
\hline $18: 40$ & $1: 18$ & 44 & 90 & 498 & 1999 & 430 & 308.7 & \\
\hline $18: 42$ & $1: 20$ & 43 & 89 & 478 & 1985 & 466 & 334.5 & Begin Steady-state \\
\hline $18: 44$ & $1: 22$ & 38 & 90 & 489 & 1989 & 466 & 334.5 & \\
\hline $18: 46$ & $1: 24$ & 41 & 90 & 510 & 1998 & 444 & 318.7 & \\
\hline $18: 48$ & $1: 26$ & 47 & 90 & 547 & 1986 & 457 & 328.0 & \\
\hline $18: 50$ & $1: 28$ & 47 & 90 & 512 & 1996 & 478 & 343.1 & \\
\hline
\end{tabular}


MATR_1.XLS

\begin{tabular}{|c|c|c|c|c|c|c|c|c|c|}
\hline $\begin{array}{c}\text { TIME OF } \\
\text { DAY }\end{array}$ & $\begin{array}{l}\text { ELAPSED } \\
\text { TIME }\end{array}$ & $\begin{array}{c}\text { FEED } \\
\text { PRESSURE }\end{array}$ & FEED TEMP & FEED FLOW & $\begin{array}{l}\text { ROTOR } \\
\text { SPEED }\end{array}$ & $\begin{array}{c}\text { PERMEATE } \\
\text { FLOW }\end{array}$ & $\begin{array}{c}\text { PERMEATE } \\
\text { FLUX }\end{array}$ & & \\
\hline $18: 52$ & $1: 30$ & 44 & 90 & 515 & 1996 & 433 & 310.8 & & \\
\hline $18: 54$ & $1: 32$ & 46 & 89 & 543 & 1985 & 462 & 331.6 & & \\
\hline $18: 56$ & $1: 34$ & 40 & 90 & 528 & 1995 & 478 & 343.1 & & \\
\hline $18: 58$ & $1: 36$ & 43 & 90 & 524 & 1987 & 436 & 313.0 & & \\
\hline $19: 00$ & $1: 38$ & 42 & 89 & 508 & 1997 & 466 & 334.5 & & \\
\hline $19: 02$ & $1: 40$ & 44 & 90 & 504 & 1992 & 469 & 336.7 & & \\
\hline $19: 04$ & $1: 42$ & 45 & 90 & 504 & 1992 & 434 & 311.5 & & \\
\hline $19: 06$ & $1: 44$ & 44 & 89 & 486 & 1987 & 451 & 323.7 & & \\
\hline $19: 08$ & $1: 46$ & 44 & 90 & 481 & 1990 & 484 & 347.4 & & \\
\hline $19: 10$ & $1: 48$ & 41 & 91 & 526 & 1990 & 418 & 300.1 & & \\
\hline $19: 12$ & $1: 50$ & 45 & 89 & 532 & 1995 & 445 & 319.4 & & \\
\hline $19: 14$ & $1: 52$ & 44 & 90 & 516 & 1989 & 477 & 342.4 & & \\
\hline $19: 16$ & $1: 54$ & 48 & 91 & 499 & 1990 & 424 & 304.4 & & \\
\hline $19: 18$ & $1: 56$ & 41 & 90 & 521 & 1998 & 442 & 317.3 & & \\
\hline $19: 20$ & $1: 58$ & 42 & 89 & 509 & 1993 & 447 & 320.9 & & \\
\hline $19: 22$ & $2: 00$ & 43 & 92 & 505 & 1987 & 434 & 311.5 & & \\
\hline $19: 24$ & $2: 02$ & 44 & 90 & 500 & 1996 & 433 & 310.8 & & \\
\hline $19: 26$ & $2: 04$ & 47 & 89 & 487 & 1996 & 443 & 318.0 & & \\
\hline $19: 28$ & $2: 06$ & 41 & 91 & 510 & 1994 & 462 & 331.6 & & \\
\hline $19: 30$ & $2: 08$ & 41 & 90 & 524 & 1986 & 412 & 295.7 & & \\
\hline $19: 32$ & $2: 10$ & 42 & 89 & 489 & 2000 & 437 & 313.7 & & \\
\hline $19: 34$ & $2: 12$ & 44 & 90 & 528 & 1990 & 451 & 323.7 & & \\
\hline $19: 36$ & $2: 14$ & 43 & 90 & 538 & 1998 & 420 & 301.5 & & \\
\hline $19: 38$ & $2: 16$ & $\overline{41}$ & 89 & 509 & 1986 & 441 & 316.6 & & \\
\hline $19: 40$ & $2: 18$ & 49 & 90 & 462 & 1993 & 459 & 329.5 & & \\
\hline $19: 42$ & $2: 20$ & 43 & 91 & 482 & 1990 & 422 & 302.9 & & \\
\hline $19: 44$ & $2: 22$ & 48 & 90 & 553 & 1998 & 440 & 315.8 & & \\
\hline $19: 46$ & $2: 24$ & 46 & 90 & 529 & 1998 & 447 & 320.9 & & \\
\hline $19: 48$ & $2: 26$ & 43 & 91 & 466 & 1984 & 420 & 301.5 & & \\
\hline $19: 50$ & $2: 28$ & 43 & 89 & 473 & 1987 & 447 & 320.9 & & \\
\hline $19: 52$ & $2: 31$ & 47 & 90 & 470 & 1993 & 463 & 332.4 & & \\
\hline $19: 54$ & $2: 33$ & 48 & 90 & 494 & 1996 & 415 & 297.9 & & \\
\hline $19: 56$ & $2: 35$ & 41 & 89 & 526 & 1987 & 451 & 323.7 & & \\
\hline $19: 58$ & $2: 37$ & 42 & 90 & 527 & 1988 & 450 & 323.0 & & \\
\hline $20: 00$ & $2: 39$ & 47 & 90 & 475 & 1989 & 421 & 302.2 & & \\
\hline $20: 02$ & $2: 41$ & 42 & 89 & 531 & 1996 & 446 & 320.2 & & \\
\hline $20: 04$ & $2: 43$ & 43 & 90 & 542 & 1988 & 441 & 316.6 & & \\
\hline $20: 06$ & $2: 45$ & 47 & 91 & 482 & 1986 & 417 & 299.3 & & \\
\hline $20: 08$ & $2: 47$ & 43 & 89 & 504 & 1996 & 405 & 290.7 & & \\
\hline $20: 10$ & $2: 49$ & 48 & 89 & 535 & 1985 & 436 & 313.0 & & \\
\hline $20: 12$ & $2: 51$ & 44 & 91 & 527 & 2003 & 435 & 312.3 & & \\
\hline $20: 14$ & $2: 53$ & 45 & 89 & 513 & 1989 & 407 & 292.2 & & \\
\hline $20: 16$ & $2: 55$ & 47 & 89 & 566 & 1983 & 446 & 320.2 & & \\
\hline $20: 18$ & $2: 57$ & 44 & 91 & 486 & 1999 & 428 & 307.2 & & \\
\hline $20: 20$ & $2: 59$ & 43 & 90 & 502 & 1984 & 397 & 285.0 & & \\
\hline $20: 23$ & $3: 01$ & 45 & 89 & 509 & 1987 & 439 & 315.1 & & \\
\hline $20: 25$ & $3: 03$ & 43 & 90 & 491 & 1989 & 449 & 322.3 & & \\
\hline $20: 27$ & $3: 05$ & 41 & 91 & 539 & 1993 & 406 & 291.4 & & \\
\hline $20: 29$ & 3:07 & 42 & 89 & 517 & 1988 & 434 & 311.5 & & \\
\hline $20: 31$ & $3: 09$ & 44 & 91 & 485 & 1987 & 455 & 326.6 & & \\
\hline
\end{tabular}


MATR_1.XLS

\begin{tabular}{|c|c|c|c|c|c|c|c|c|}
\hline $\begin{array}{l}\text { TIME OF } \\
\text { DAY }\end{array}$ & $\begin{array}{c}\text { ELAPSED } \\
\text { TIME }\end{array}$ & \begin{tabular}{|c|} 
FEED \\
PRESSURE
\end{tabular} & FEED TEMP & FEED FLOW & $\begin{array}{l}\text { ROTOR } \\
\text { SPEED }\end{array}$ & \begin{tabular}{|c|} 
PERMEATE \\
FLOW
\end{tabular} & $\begin{array}{l}\text { PERMEATE } \\
\text { FLUX }\end{array}$ & \\
\hline $20: 33$ & $3: 11$ & 44 & 91 & 511 & 1988 & 412 & 295.7 & \\
\hline $20: 35$ & $3: 13$ & 40 & 89 & 470 & 1986 & 428 & 307.2 & \\
\hline $20: 37$ & $3: 15$ & 43 & 90 & 503 & 1985 & 432 & 310.1 & \\
\hline $20: 39$ & $3: 17$ & 41 & 91 & 490 & 1992 & 424 & 304.4 & \\
\hline $20: 41$ & $3: 19$ & 44 & 90 & 524 & 1986 & 421 & 302.2 & End Steady-state \\
\hline \multicolumn{2}{|c|}{ Steady-state Avg. } & 44 & 90 & 508 & 1991 & 440 & 316 & \\
\hline \multicolumn{2}{|c|}{ Maximum } & 49 & 92 & 566 & 2003 & 484 & 347 & \\
\hline Minimum & & 38 & 89 & 462 & 1983 & 397 & 285 & \\
\hline \multicolumn{2}{|c|}{ Standard Deviation } & 2.4 & 0.8 & 23.5 & 4.9 & 20.1 & 14.4 & \\
\hline & & & & & & & & \\
\hline \multicolumn{4}{|c|}{ TEST CONDITIONS: MATR_1.XLS } & Temp. $=$ & $90 \mathrm{~F}$ & & & \\
\hline & & & & Press. $=$ & 45 psig & & & \\
\hline \multicolumn{2}{|c|}{ Date Run: 9-May-96 } & & & Rotor $=$ & $2000 \mathrm{rpm}$ & & & \\
\hline & & & & Solids $=$ & $15 w t \%$ & & & \\
\hline & & & & Flow $=$ & $500 \mathrm{~L} / \mathrm{hr}$ & & & \\
\hline
\end{tabular}




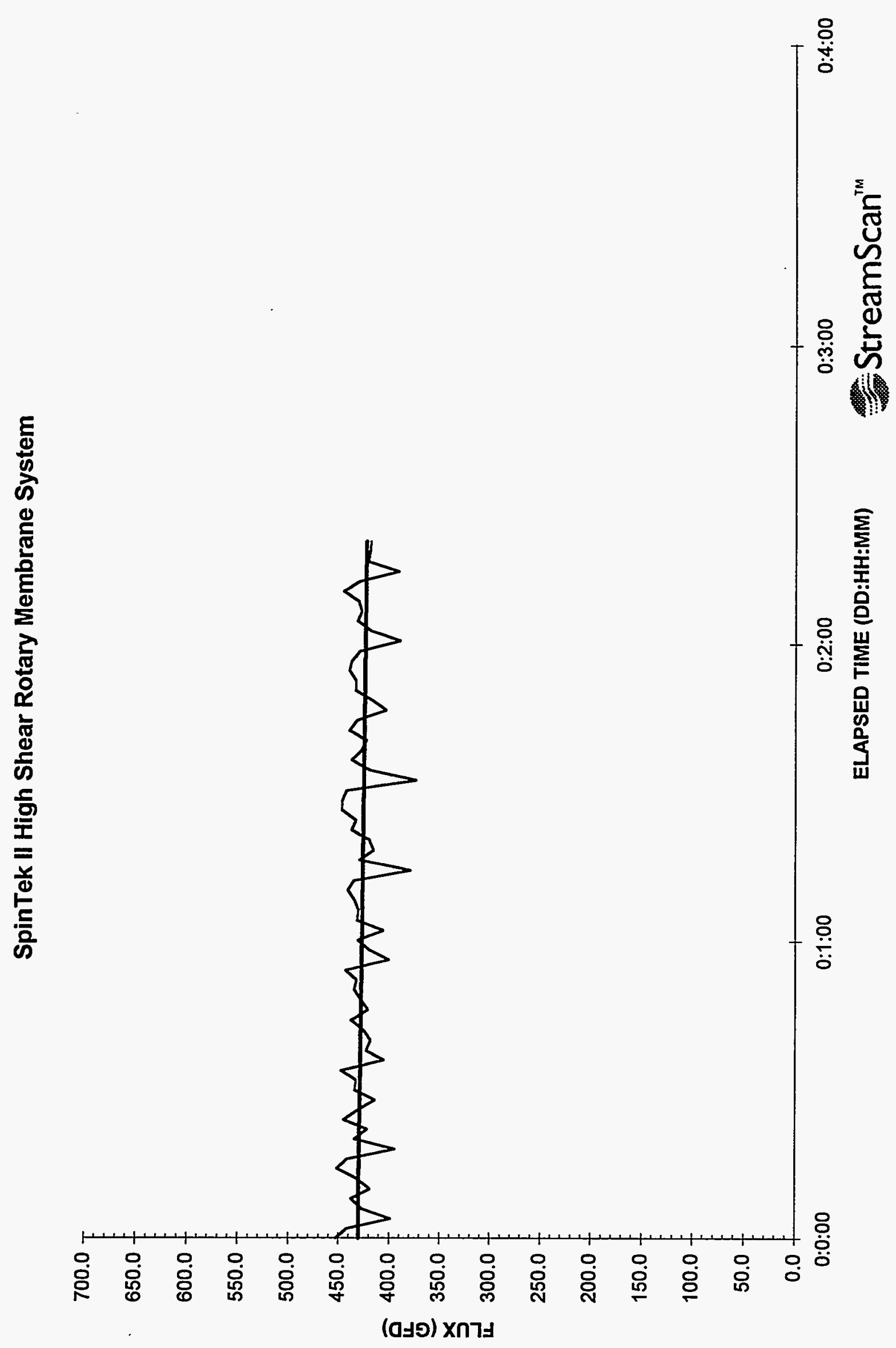




\begin{tabular}{|c|c|c|c|c|c|c|c|c|}
\hline $\begin{array}{c}\text { TIME OF } \\
\text { DAY }\end{array}$ & $\begin{array}{c}\text { ELAPSED } \\
\text { TIME }\end{array}$ & $\begin{array}{c}\text { FEED } \\
\text { PRESSURE }\end{array}$ & FEED TEMP & FEED FLOW & $\begin{array}{l}\text { ROTOR } \\
\text { SPEED }\end{array}$ & $\begin{array}{c}\text { PERMEATE } \\
\text { FLOW }\end{array}$ & $\begin{array}{c}\text { PERMEATE } \\
\text { FLUX }\end{array}$ & \\
\hline $17: 15$ & $0: 00$ & 75 & 120 & 475 & 1501 & 630 & 452.2 & Begin Steady-state \\
\hline $17: 17$ & $0: 01$ & 75 & 120 & 455 & 1507 & 616 & 442.2 & \\
\hline $17: 19$ & $0: 03$ & 75 & 120 & 515 & 1500 & 555 & 398.4 & \\
\hline $17: 21$ & $0: 05$ & 77 & 118 & 501 & 1500 & 595 & 427.1 & \\
\hline $17: 23$ & $0: 07$ & 73 & 118 & 484 & 1508 & 609 & 437.2 & \\
\hline $17: 25$ & $0: 09$ & 76 & 118 & 492 & 1496 & 584 & 419.2 & \\
\hline $17: 27$ & $0: 12$ & 73 & 119 & 455 & 1498 & 602 & 432.1 & \\
\hline $17: 29$ & $0: 14$ & 74 & 120 & 455 & 1504 & 629 & 451.5 & \\
\hline $17: 31$ & $0: 16$ & 73 & 120 & 479 & 1506 & 615 & 441.5 & \\
\hline $17: 33$ & $0: 18$ & 79 & 119 & 485 & 1495 & 549 & 394.1 & \\
\hline $17: 36$ & $0: 20$ & 73 & 117 & 440 & 1506 & 605 & 434.3 & \\
\hline $17: 37$ & $0: 22$ & 80 & 118 & 464 & 1500 & 588 & 422.1 & \\
\hline $17: 40$ & $0: 24$ & 80 & 118 & 442 & 1499 & 620 & 445.1 & \\
\hline $17: 41$ & $0: 26$ & 73 & 118 & 428 & 1503 & 601 & 431.4 & \\
\hline $17: 44$ & $0: 28$ & 74 & 119 & 497 & 1503 & 577 & 414.2 & \\
\hline $17: 45$ & $0: 30$ & 78 & 120 & 481 & 1503 & 605 & 434.3 & \\
\hline $17: 48$ & $0: 32$ & 74 & 120 & 433 & 1507 & 603 & 432.9 & \\
\hline $17: 50$ & $0: 34$ & 76 & 120 & 484 & 1504 & 624 & 447.9 & \\
\hline $17: 52$ & $0: 36$ & 74 & 118 & 450 & 1497 & 565 & 405.6 & \\
\hline $17: 54$ & $0: 38$ & 71 & 117 & 455 & 1500 & 589 & 422.8 & \\
\hline $17: 56$ & $0: 40$ & 75 & 118 & 458 & 1495 & 583 & 418.5 & \\
\hline $17: 58$ & $0: 42$ & 76 & 117 & 447 & 1500 & 593 & 425.7 & \\
\hline $18: 00$ & $0: 44$ & 78 & 118 & 466 & 1496 & 610 & 437.9 & \\
\hline $18: 02$ & $0: 46$ & 72 & 118 & 514 & 1504 & 587 & 421.4 & \\
\hline $18: 04$ & $0: 48$ & 72 & 118 & 504 & 1504 & 597 & 428.5 & \\
\hline $18: 06$ & $0: 50$ & 72 & 119 & 449 & 1499 & 606 & 435.0 & \\
\hline $18: 08$ & $0: 52$ & 72 & 120 & 479 & 1499 & 603 & 432.9 & \\
\hline $18: 10$ & $0: 54$ & 70 & 120 & 449 & 1506 & 618 & 443.6 & \\
\hline $18: 12$ & $0: 56$ & 75 & 120 & 516 & 1507 & 558 & 400.5 & \\
\hline $18: 14$ & $0: 58$ & 74 & 117 & 499 & 1504 & 585 & 419.9 & \\
\hline $18: 16$ & $1: 00$ & 71 & 117 & 449 & 1502 & 601 & 431.4 & \\
\hline $18: 18$ & $1: 02$ & 73 & 118 & 456 & 1499 & 566 & 406.3 & \\
\hline $18: 20$ & $1: 04$ & 75 & 118 & 509 & 1504 & 602 & 432.1 & \\
\hline $18: 22$ & $1: 06$ & 74 & 119 & 461 & 1503 & 600 & 430.7 & \\
\hline $18: 24$ & $1: 08$ & 73 & 119 & 445 & 1504 & 606 & 435.0 & \\
\hline $18: 26$ & $1: 10$ & 74 & 119 & 458 & 1496 & 615 & 441.5 & \\
\hline $18: 28$ & $1: 12$ & 71 & 120 & 491 & 1500 & 607 & 435.7 & \\
\hline $18: 30$ & $1: 14$ & 77 & 119 & 485 & 1505 & 529 & 379.7 & \\
\hline $18: 32$ & $1: 16$ & 72 & 117 & 406 & 1496 & 599 & 430.0 & \\
\hline $18: 34$ & $1: 18$ & 75 & 118 & 484 & 1497 & 580 & 416.3 & \\
\hline $18: 36$ & $1: 20$ & 78 & 118 & 482 & 1506 & 586 & 420.6 & \\
\hline $18: 38$ & $1: 22$ & 72 & 118 & 442 & 1509 & 610 & 437.9 & \\
\hline $18: 40$ & $1: 24$ & 72 & 119 & 480 & 1501 & 604 & 433.6 & \\
\hline $18: 42$ & $1: 26$ & 74 & 119 & 509 & 1503 & 623 & 447.2 & \\
\hline $18: 44$ & $1: 28$ & 75 & 120 & 421 & 1500 & 623 & 447.2 & \\
\hline
\end{tabular}


MATR_2.XLS

\begin{tabular}{|c|c|c|c|c|c|c|c|c|}
\hline $\begin{array}{c}\text { TIME OF } \\
\text { DAY }\end{array}$ & $\begin{array}{c}\text { ELAPSED } \\
\text { TIME }\end{array}$ & \begin{tabular}{|c|} 
FEED \\
PRESSURE \\
\end{tabular} & FEED TEMP & FEED FLOW & $\begin{array}{l}\text { ROTOR } \\
\text { SPEED }\end{array}$ & \begin{tabular}{|c|}
$\begin{array}{c}\text { PERMEATE } \\
\text { FLOW }\end{array}$ \\
\end{tabular} & $\begin{array}{l}\text { PERMEATE } \\
\text { FLUX }\end{array}$ & \\
\hline $18: 46$ & $1: 30$ & 76 & 120 & 478 & 1503 & 617 & 442.9 & \\
\hline $18: 48$ & $1: 32$ & 79 & 119 & 513 & 1496 & 521 & 374.0 & \\
\hline $18: 50$ & $1: 34$ & 73 & 117 & 464 & 1494 & 586 & 420.6 & \\
\hline $18: 52$ & $1: 36$ & 72 & 117 & 489 & 1504 & 610 & 437.9 & \\
\hline $18: 54$ & $1: 38$ & 76 & 117 & 454 & 1503 & 597 & 428.5 & \\
\hline 18:56 & $1: 40$ & 71 & 118 & 480 & 1496 & 590 & 423.5 & \\
\hline $18: 58$ & $1: 42$ & 77 & 119 & 428 & 1503 & 613 & 440.0 & \\
\hline $19: 00$ & $1: 44$ & 75 & 120 & 453 & 1496 & 603 & 432.9 & \\
\hline 19:02 & $1: 46$ & 74 & 120. & 554 & 1510 & 563 & 404.1 & - \\
\hline $19: 04$ & $1: 48$ & 73 & 118 & 484 & 1503 & 582 & 417.8 & \\
\hline $19: 06$ & $1: 50$ & 78 & 117 & 448 & 1504 & 605 & 434.3 & \\
\hline $19: 08$ & $1: 52$ & 73 & 118 & 475 & 1508 & 604 & 433.6 & \\
\hline $19: 10$ & $1: 54$ & 74 & 119 & 408 & 1496 & 613 & 440.0 & \\
\hline $19: 12$ & $1: 56$ & 76 & 119 & 514 & 1503 & 610 & 437.9 & \\
\hline $19: 14$ & $1: 58$ & 76 & 120 & 389 & 1507 & 599 & 430.0 & \\
\hline $19: 16$ & $2: 00$ & 74 & 118 & 507 & 1504 & 543 & 389.8 & \\
\hline $19: 18$ & $2: 02$ & 74 & 117 & 503 & 1496 & 584 & 419.2 & \\
\hline $19: 20$ & $2: 04$ & 75 & 117 & 481 & 1493 & 602 & 432.1 & \\
\hline $19: 22$ & $2: 06$ & 77 & 118 & 447 & 1500 & 596 & 427.8 & \\
\hline $19: 24$ & $2: 08$ & 73 & 119 & 501 & 1496 & 601 & 431.4 & \\
\hline $19: 26$ & $2: 10$ & 76 & 119 & 426 & 1496 & 621 & 445.8 & \\
\hline $19: 28$ & $2: 12$ & 75 & 120 & 489 & 1502 & 600 & 430.7 & \\
\hline $19: 30$ & $2: 14$ & 75 & 119 & 496 & 1503 & 545 & 391.2 & \\
\hline $19: 32$ & $2: 16$ & 76 & 117 & 411 & 1488 & 587 & 421.4 & \\
\hline $19: 34$ & $2: 18$ & 79 & 118 & 427 & 1503 & 585 & 419.9 & \\
\hline $19: 36$ & $2: 20$ & 74 & 118 & 488 & 1505 & 584 & 419.2 & End Steady-state \\
\hline \multicolumn{2}{|c|}{ Steady-state Avg. } & 75 & 119 & 470 & 1501 & 595 & 427 & \\
\hline \multicolumn{2}{|l|}{ Maximum } & 80 & 120 & 554 & 1510 & 630 & 452 & \\
\hline Minimum & & 70 & 117 & 389 & 1488 & 521 & 374 & \\
\hline \multicolumn{2}{|c|}{ Standard Deviation } & 2.3 & 1.1 & 31.5 & 4.4 & 22.7 & 16.3 & \\
\hline & & & & & & & & \\
\hline \multicolumn{4}{|c|}{ TEST CONDITIONS: MATR_2.XLS } & Temp. $=$ & $120 \mathrm{~F}$ & & & \\
\hline & & & & Press. $=$ & $75 \mathrm{psig}$ & & & \\
\hline \multicolumn{3}{|c|}{ Date Run: 10-May-96 } & & Rotor $=$ & $1500 \mathrm{rpm}$ & & & \\
\hline & & & & Solids $=$ & $15 w t \%$ & & & \\
\hline & & & & Flow $=$ & $500 \mathrm{~L} / \mathrm{hr}$ & & & \\
\hline
\end{tabular}


SpinTek II High Shear Rotary Membrane System

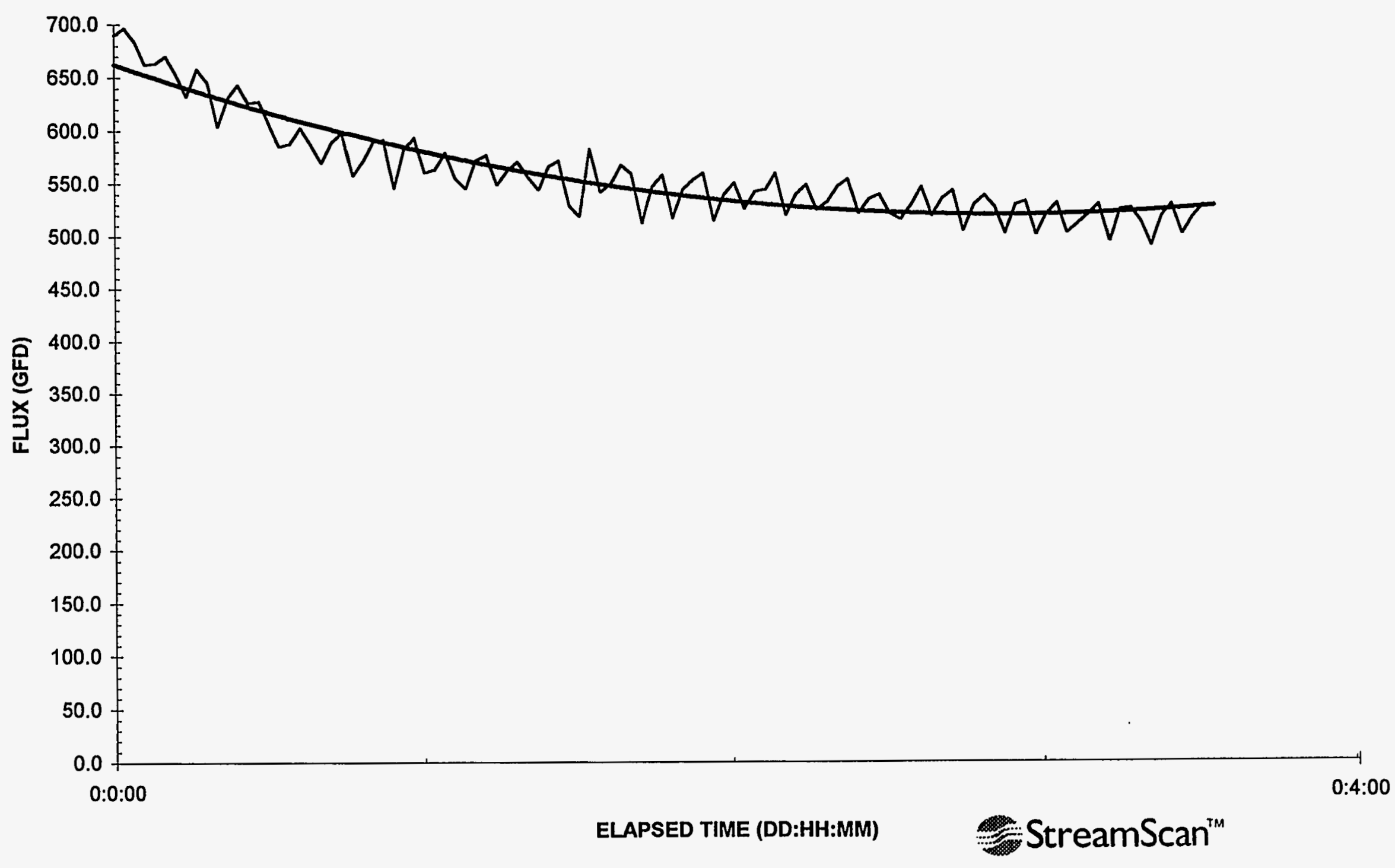




\begin{tabular}{|c|c|c|c|c|c|c|c|c|c|}
\hline $\begin{array}{c}\text { TIME OF } \\
\text { DAY }\end{array}$ & $\begin{array}{c}\text { ELAPSED } \\
\text { TIME }\end{array}$ & $\begin{array}{c}\text { FEED } \\
\text { PRESSURE }\end{array}$ & FEED TEMP & FEED FLOW & $\begin{array}{l}\text { ROTOR } \\
\text { SPEED }\end{array}$ & $\begin{array}{c}\text { PERMEATE } \\
\text { FLOW }\end{array}$ & $\begin{array}{c}\text { PERMEATE } \\
\text { FLUX }\end{array}$ & & \\
\hline $12: 04$ & $0: 00$ & 66 & 90 & 467 & 995 & 961 & 689.8 & & \\
\hline $12: 06$ & $0: 02$ & 59 & 91 & 483 & 997 & 970 & 696.3 & & \\
\hline $12: 08$ & $0: 04$ & 62 & 91 & 472 & 997 & 952 & 683.4 & & \\
\hline $12: 11$ & $0: 06$ & 60 & 91 & 476 & 995 & 922 & 661.8 & & \\
\hline $12: 13$ & $0: 08$ & 63 & 90 & 477 & 995 & 924 & 663.3 & & \\
\hline $12: 15$ & $0: 10$ & 67 & 90 & 477 & 995 & 933 & 669.7 & & \\
\hline $12: 17$ & $0: 12$ & 67 & 91 & 478 & 996 & 910 & 653.2 & & \\
\hline $12: 19$ & $0: 14$ & 67 & 90 & 480 & 998 & 880 & 631.7 & & \\
\hline $12: 21$ & $0: 16$ & 59 & 89 & 474 & 997 & 916 & 657.5 & & \\
\hline $12: 23$ & $0: 18$ & 59 & 91 & 478 & 997 & 899 & 645.3 & & \\
\hline $12: 25$ & $0: 20$ & 64 & 90 & 480 & 996 & 841 & 603.7 & & \\
\hline $12: 27$ & $0: 22$ & 58 & 89 & 485 & 997 & 879 & 631.0 & & \\
\hline $12: 29$ & $0: 24$ & 60 & 90 & 483 & 997 & 895 & 642.5 & & \\
\hline $12: 31$ & $0: 26$ & 64 & 91 & 487 & 995 & 872 & 625.9 & & \\
\hline $12: 33$ & $0: 28$ & 65 & 90 & 485 & 996 & 874 & 627.4 & & \\
\hline $12: 35$ & $0: 30$ & 56 & 90 & 491 & 995 & 843 & 605.1 & & \\
\hline $12: 37$ & $0: 32$ & 62 & 90 & 494 & 996 & 815 & 585.0 & & \\
\hline $12: 39$ & $0: 34$ & 60 & 90 & 486 & 995 & 818 & 587.2 & & \\
\hline $12: 41$ & $0: 36$ & 60 & 90 & 497 & 997 & 839 & 602.3 & & \\
\hline $12: 43$ & $0: 38$ & 57 & 91 & 491 & 997 & 818 & 587.2 & & \\
\hline $12: 45$ & $0: 40$ & 64 & 90 & 489 & 997 & 793 & 569.2 & & \\
\hline $12: 47$ & $0: 42$ & 56 & 89 & 499 & 995 & 820 & 588.6 & & \\
\hline $12: 49$ & $0: 44$ & 63 & 90 & 495 & 996 & 833 & 598.0 & & \\
\hline $12: 51$ & $0: 46$ & 60 & 91 & 491 & 995 & 776 & 557.0 & & \\
\hline $12: 53$ & $0: 48$ & 57 & 89 & 495 & 995 & 795 & 570.7 & & \\
\hline $12: 55$ & $0: 50$ & 63 & 89 & 494 & 993 & 821 & 589.3 & & \\
\hline $12: 57$ & $0: 52$ & 61 & 90 & 486 & 996 & 823 & 590.8 & & \\
\hline $12: 59$ & $0: 54$ & 61 & 90 & 492 & 996 & 760 & 545.5 & & \\
\hline 13:01 & $0: 56$ & 64 & 89 & 492 & 994 & 811 & 582.2 & & \\
\hline $13: 03$ & $0: 58$ & 61 & 90 & 492 & 997 & 826 & 592.9 & & \\
\hline $13: 05$ & $1: 00$ & 61 & 90 & 486 & 996 & 780 & 559.9 & & \\
\hline $13: 07$ & $1: 02$ & 57 & 89 & 488 & 994 & 784 & 562.8 & & \\
\hline $13: 09$ & $1: 04$ & 59 & 89 & 486 & 994 & 806 & 578.6 & & \\
\hline $13: 11$ & $1: 06$ & 60 & 90 & 482 & 996 & 773 & 554.9 & & \\
\hline $13: 13$ & $1: 08$ & 59 & 89 & 495 & 998 & 759 & 544.8 & & \\
\hline $13: 15$ & $1: 10$ & 61 & 89 & 488 & 994 & 796 & 571.4 & & \\
\hline $13: 17$ & $1: 12$ & 61 & 90 & 487 & 996 & 803 & 576.4 & & \\
\hline $13: 19$ & $1: 14$ & 63 & 90 & 487 & 996 & 764 & 548.4 & & \\
\hline $13: 21$ & $1: 16$ & 59 & 89 & 487 & 994 & 783 & 562.1 & & \\
\hline $13: 23$ & $1: 18$ & 59 & 89 & 487 & 996 & 794 & 570.0 & & \\
\hline $13: 25$ & $1: 20$ & 63 & 90 & 483 & 993 & 774 & 555.6 & & \\
\hline $13: 27$ & $1: 22$ & 60 & 89 & 491 & 997 & 757 & 543.4 & & \\
\hline $13: 29$ & $1: 24$ & 65 & 89 & 496 & 994 & 788 & 565.6 & & \\
\hline $13: 31$ & $1: 26$ & 60 & 90 & 489 & 995 & 795 & 570.7 & & \\
\hline $13: 33$ & $1: 28$ & 59 & 90 & 490 & 1006 & 736 & 528.3 & & \\
\hline
\end{tabular}


MATR_3.XLS

\begin{tabular}{|c|c|c|c|c|c|c|c|c|}
\hline $\begin{array}{l}\text { TIME OF } \\
\text { DAY }\end{array}$ & $\begin{array}{l}\text { ELAPSED } \\
\text { TIME }\end{array}$ & $\begin{array}{c}\text { FEED } \\
\text { PRESSURE }\end{array}$ & FEED TEMP & FEED FLOW & $\begin{array}{l}\text { ROTOR } \\
\text { SPEED }\end{array}$ & $\begin{array}{c}\text { PERMEATE } \\
\text { FLOW }\end{array}$ & $\begin{array}{c}\text { PERMEATE } \\
\text { FLUX }\end{array}$ & \\
\hline $13: 35$ & $1: 30$ & 61 & 89 & 496 & 995 & 722 & 518.3 & \\
\hline $13: 37$ & $1: 32$ & 61 & 89 & 496 & 994 & 810 & 581.4 & \\
\hline $13: 39$ & $1: 34$ & 58 & 90 & 487 & 995 & 754 & 541.2 & \\
\hline $13: 41$ & $1: 36$ & 61 & 89 & 499 & 995 & 765 & 549.1 & \\
\hline $13: 43$ & $1: 38$ & 61 & 90 & 492 & 997 & 789 & 566.4 & \\
\hline $13: 45$ & $1: 40$ & 61 & 90 & 488 & 993 & 778 & 558.5 & \\
\hline $13: 47$ & $1: 42$ & 59 & 89 & 483 & 993 & 713 & 511.8 & \\
\hline $13: 49$ & $1: 44$ & 58 & 89 & 496 & 995 & 761 & 546.3 & Begin Steady-state \\
\hline $13: 51$ & $1: 46$ & 61 & 90 & 500 & 992 & 776 & 557.0 & \\
\hline $13: 53$ & $1: 48$ & 57 & 90 & 482 & 993 & 719 & 516.1 & \\
\hline $13: 55$ & $1: 50$ & 63 & 89 & 482 & 995 & 757 & 543.4 & \\
\hline $13: 57$ & $1: 52$ & 61 & 89 & 484 & 993 & 770 & 552.7 & \\
\hline $13: 59$ & $1: 54$ & 61 & 90 & 487 & 999 & 779 & 559.2 & \\
\hline $14: 01$ & $1: 56$ & 61 & 90 & 475 & 997 & 716 & 514.0 & \\
\hline $14: 03$ & $1: 58$ & 62 & 89 & 497 & 993 & 751 & 539.1 & \\
\hline $14: 05$ & $2: 00$ & 60 & 89 & 488 & 995 & 766 & 549.9 & \\
\hline $14: 07$ & $2: 02$ & 63 & 91 & 490 & 996 & 732 & 525.5 & \\
\hline $14: 09$ & $2: 04$ & 61 & 89 & 496 & 993 & 754 & 541.2 & \\
\hline $14: 11$ & $2: 06$ & 61 & 89 & 492 & 996 & 757 & 543.4 & \\
\hline $14: 13$ & $2: 08$ & 63 & 90 & 482 & 992 & 778 & 558.5 & \\
\hline $14: 15$ & $2: 10$ & 58 & 90 & 495 & 995 & 723 & 519.0 & \\
\hline $14: 17$ & $2: 12$ & 63 & 89 & 492 & 998 & 750 & 538.4 & \\
\hline $14: 19$ & $2: 14$ & 62 & 89 & 482 & 997 & 763 & 547.7 & \\
\hline $14: 21$ & $2: 16$ & 62 & 90 & 498 & 995 & 730 & 524.0 & \\
\hline $14: 23$ & $2: 18$ & 58 & 89 & 493 & 994 & 741 & 531.9 & \\
\hline $14: 25$ & $2: 20$ & 60 & 89 & 476 & 994 & 761 & 546.3 & \\
\hline $14: 27$ & $2: 22$ & 58 & 90 & 490 & 999 & 770 & 552.7 & \\
\hline $14: 29$ & $2: 24$ & 63 & 90 & 478 & 995 & 725 & 520.4 & \\
\hline $14: 31$ & $2: 26$ & 58 & 89 & 499 & 995 & 744 & 534.1 & \\
\hline $14: 33$ & $2: 28$ & 61 & 89 & 500 & 995 & 750 & 538.4 & \\
\hline $14: 35$ & $2: 30$ & 62 & 90 & 490 & 996 & 725 & 520.4 & \\
\hline $14: 37$ & $2: 32$ & 59 & 89 & 477 & 994 & 717 & 514.7 & \\
\hline $14: 39$ & $2: 34$ & 65 & 89 & 494 & 994 & 737 & 529.0 & \\
\hline $14: 41$ & $2: 36$ & 57 & 90 & 496 & 994 & 760 & 545.5 & \\
\hline $14: 43$ & $2: 38$ & 66 & 91 & 475 & 993 & 722 & 518.3 & \\
\hline $14: 45$ & $2: 40$ & 61 & 89 & 491 & 993 & 745 & 534.8 & \\
\hline $14: 47$ & $2: 42$ & 61 & 89 & 499 & 993 & 755 & 542.0 & \\
\hline $14: 49$ & $2: 45$ & 63 & 90 & 495 & 992 & 702 & 503.9 & \\
\hline $14: 51$ & $2: 47$ & 61 & 89 & 486 & 996 & 736 & 528.3 & \\
\hline $14: 53$ & $2: 49$ & 63 & 89 & 489 & 994 & 749 & 537.7 & \\
\hline $14: 55$ & $2: 51$ & 60 & 90 & 490 & 994 & 734 & 526.9 & \\
\hline $14: 57$ & $2: 53$ & 61 & 89 & 484 & 995 & 698 & 501.0 & \\
\hline $15: 00$ & $2: 55$ & 63 & 89 & 485 & 996 & 736 & 528.3 & \\
\hline $15: 01$ & $2: 57$ & 60 & 90 & 482 & 994 & 741 & 531.9 & \\
\hline $15: 04$ & $2: 59$ & 58 & 90 & 493 & 998 & 697 & 500.3 & \\
\hline $15: 06$ & $3: 01$ & 59 & 89 & 486 & 992 & 724 & 519.7 & \\
\hline $15: 08$ & $3: 03$ & 62 & 89 & 497 & 991 & 738 & 529.8 & \\
\hline $15: 10$ & $3: 05$ & 62 & 90 & 486 & 996 & 699 & 501.8 & \\
\hline $15: 12$ & $3: 07$ & 62 & 89 & 483 & 996 & 711 & 510.4 & \\
\hline $15: 14$ & $3: 09$ & 58 & 89 & 492 & 995 & 724 & 519.7 & \\
\hline
\end{tabular}


MATR_3.XLS

\begin{tabular}{|c|c|c|c|c|c|c|c|c|}
\hline $\begin{array}{c}\text { TIME OF } \\
\text { DAY }\end{array}$ & $\begin{array}{c}\text { ELAPSED } \\
\text { TIME }\end{array}$ & \begin{tabular}{|c|} 
FEED \\
PRESSURE \\
\end{tabular} & FEED TEMP & FEED FLOW & $\begin{array}{l}\text { ROTOR } \\
\text { SPEED }\end{array}$ & \begin{tabular}{|c|}
$\begin{array}{c}\text { PERMEATE } \\
\text { FLOW }\end{array}$ \\
\end{tabular} & \begin{tabular}{|c|} 
PERMEATE \\
FLUX
\end{tabular} & \\
\hline $15: 16$ & $3: 11$ & 60 & 90 & 480 & 997 & 737 & 529.0 & \\
\hline $15: 18$ & $3: 13$ & 59 & 90 & 483 & 995 & 688 & 493.9 & \\
\hline $15: 20$ & $3: 15$ & 64 & 89 & 497 & 994 & 730 & 524.0 & \\
\hline $15: 22$ & $3: 17$ & 57 & 89 & 492 & 994 & 732 & 525.5 & \\
\hline $15: 24$ & $3: 19$ & 58 & 90 & 486 & 997 & 715 & 513.2 & \\
\hline $15: 26$ & $3: 21$ & 65 & 90 & 491 & 994 & 682 & 489.6 & \\
\hline $15: 28$ & $3: 23$ & 61 & 89 & 485 & 992 & 721 & 517.6 & \\
\hline $15: 30$ & $3: 25$ & 62 & 90 & 485 & 996 & 736 & 528.3 & \\
\hline $15: 32$ & $3: 27$ & 63 & 91 & 482 & 997 & 697 & 500.3 & \\
\hline $15: 34$ & $3: 29$ & 62 & 89 & 480 & 995 & 719 & 516.1 & \\
\hline $15: 36$ & $3: 31$ & 61 & 89 & 487 & 992 & 735 & 527.6 & \\
\hline $15: 38$ & $3: 33$ & 59 & 90 & 487 & 995 & 734 & 526.9 & End Steady-state \\
\hline \multicolumn{2}{|c|}{ Steady-State Avg. } & 61 & 90 & 488 & 995 & 735 & 528 & \\
\hline \multicolumn{2}{|c|}{ Maximum } & 66 & 91 & 500 & 999 & 779 & 559 & \\
\hline Minimum & & 57 & 89 & 475 & 991 & 682 & 490 & \\
\hline \multicolumn{2}{|c|}{ Standard Deviation } & 2.2 & 0.6 & 6.8 & 1.8 & 23.3 & 16.7 & \\
\hline \multirow{2}{*}{\multicolumn{4}{|c|}{ TEST CONDITIONS: MATR_3.XLS }} & & $\ln 25$ & & & \\
\hline & & & & lemp. $=$ & $90 \mathrm{r}$ & & & \\
\hline \multirow{2}{*}{\multicolumn{3}{|c|}{ Date Run: 11-May-96 }} & & Press. $=$ & 60 psig & & & \\
\hline & & & & Rotor $=$ & $1000 \mathrm{rpm}$ & & & \\
\hline & & & & Solids $=$ & $1 w t \%$ & & & \\
\hline & & & & Flow $=$ & $500 \mathrm{~L} / \mathrm{hr}$ & & & \\
\hline
\end{tabular}


SpinTek II High Shear Rotary Membrane System

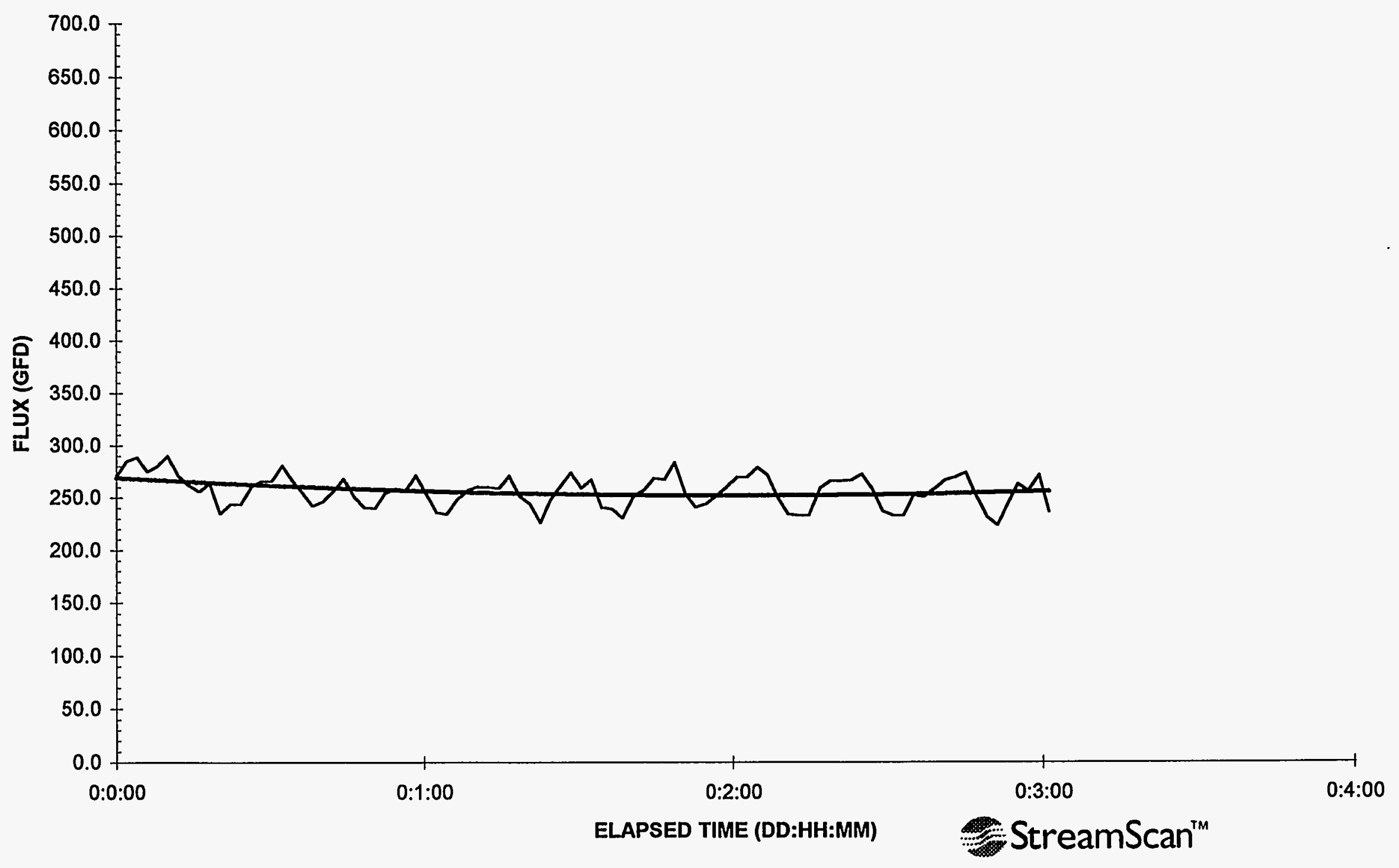


MATR_4.XLS

\begin{tabular}{|c|c|c|c|c|c|c|c|c|}
\hline $\begin{array}{l}\text { TIME OF } \\
\text { DAY }\end{array}$ & $\begin{array}{c}\text { ELAPSED } \\
\text { TIME }\end{array}$ & $\begin{array}{c}\text { FEED } \\
\text { PRESSURE }\end{array}$ & FEED TEMP & FEED FLOW & $\begin{array}{l}\text { ROTOR } \\
\text { SPEED }\end{array}$ & $\begin{array}{l}\text { PERMEATE } \\
\text { FLOW }\end{array}$ & $\begin{array}{l}\text { PERMEATE } \\
\text { FLUX }\end{array}$ & \\
\hline $7: 54$ & $0: 00$ & 62 & 88 & 510 & 1995 & 377 & 270.6 & \\
\hline $7: 56$ & $0: 02$ & 60 & 88 & 511 & 2006 & 397 & 285.0 & \\
\hline $7: 58$ & $0: 04$ & 58 & 88 & 512 & 2001 & 402 & 288.6 & \\
\hline $8: 00$ & $0: 06$ & 60 & 88 & 512 & 1997 & 383 & 274.9 & \\
\hline $8: 02$ & $0: 08$ & 60 & 89 & 512 & 2000 & 390 & 280.0 & . \\
\hline $8: 04$ & $0: 10$ & 63 & 89 & 509 & 1997 & 404 & 290.0 & \\
\hline $8: 06$ & $0: 12$ & 62 & 94 & 507 & 2005 & 378 & 271.3 & \\
\hline $8: 08$ & $0: 14$ & 58 & 93 & 508 & 2004 & 365 & 262.0 & \\
\hline $8: 10$ & $0: 16$ & 61 & 93 & 508 & 1997 & 356 & 255.5 & \\
\hline $8: 12$ & $0: 18$ & 62 & 92 & 510 & 2000 & 367 & 263.4 & \\
\hline $8: 14$ & $0: 20$ & 60 & 91 & 506 & 1996 & 327 & 234.7 & \\
\hline $8: 16$ & $0: 22$ & 59 & 91 & 504 & 1995 & 339 & 243.3 & \\
\hline $8: 18$ & $0: 24$ & 57 & 90 & 504 & 1992 & 340 & 244.1 & \\
\hline $8: 20$ & $0: 26$ & 58 & 88 & 507 & 1997 & 363 & 260.6 & \\
\hline $8: 22$ & $0: 28$ & 57 & 88 & 505 & 1992 & 370 & 265.6 & \\
\hline $8: 24$ & $0: 30$ & 60 & 89 & 506 & 1996 & 370 & 265.6 & \\
\hline $8: 26$ & $0: 32$ & 58 & 90 & 506 & 1990 & 391 & 280.7 & \\
\hline $8: 28$ & $0: 34$ & 58 & 90 & 504 & 1990 & 372 & 267.0 & \\
\hline $8: 30$ & $0: 36$ & 59 & 91 & 503 & 1996 & 355 & 254.8 & \\
\hline $8: 32$ & $0: 38$ & 57 & 90 & 502 & 2001 & 337 & 241.9 & \\
\hline $8: 34$ & $0: 40$ & 59 & 90 & 501 & 1989 & 343 & 246.2 & \\
\hline $8: 36$ & $0: 42$ & 59 & 90 & 503 & 1989 & 357 & 256.3 & \\
\hline $8: 38$ & $0: 44$ & 61 & 89 & 503 & 1995 & 373 & 267.8 & \\
\hline $8: 40$ & $0: 46$ & 57 & 91 & 501 & 1996 & 349 & 250.5 & Begin Steady-state \\
\hline $8: 42$ & $0: 48$ & 58 & 90 & 502 & 1995 & 335 & 240.5 & \\
\hline $8: 44$ & $0: 50$ & 58 & 90 & 499 & 1994 & 334 & 239.8 & \\
\hline $8: 46$ & $0: 52$ & 57 & 89 & 500 & 1994 & 354 & 254.1 & \\
\hline $8: 48$ & $0: 54$ & 60 & 89 & 501 & 1998 & 360 & 258.4 & \\
\hline $8: 50$ & $0: 56$ & 57 & 89 & 504 & 1997 & 358 & 257.0 & \\
\hline $8: 52$ & $0: 58$ & 57 & 90 & 499 & 1994 & 378 & 271.3 & \\
\hline $8: 54$ & $1: 00$ & 59 & 91 & 501 & 1993 & 353 & 253.4 & \\
\hline $8: 56$ & $1: 02$ & 58 & 91 & 495 & 1992 & 329 & 236.2 & \\
\hline $8: 58$ & $1: 04$ & 57 & 90 & 498 & 2005 & 326 & 234.0 & \\
\hline $9: 00$ & $1: 06$ & 59 & 90 & 495 & 1990 & 346 & 248.4 & \\
\hline $9: 02$ & $1: 08$ & 60 & 89 & 497 & 1993 & 358 & 257.0 & \\
\hline $9: 04$ & $1: 10$ & 60 & 89 & 496 & 1996 & 363 & 260.6 & \\
\hline $9: 06$ & $1: 12$ & 60 & 90 & 497 & 1991 & 362 & 259.9 & \\
\hline $9: 08$ & $1: 14$ & 61 & 89 & 499 & 2001 & 360 & 258.4 & \\
\hline $9: 10$ & $1: 16$ & 57 & 91 & 499 & 1994 & 377 & 270.6 & \\
\hline $9: 12$ & $1: 18$ & 58 & 91 & 496 & 1989 & 350 & 251.2 & \\
\hline $9: 14$ & $1: 20$ & 56 & 91 & 495 & 2006 & 340 & 244.1 & \\
\hline $9: 16$ & $1: 22$ & 57 & 90 & 497 & 1992 & 315 & 226.1 & \\
\hline $9: 18$ & $1: 24$ & 59 & 89 & 494 & 1996 & 345 & 247.7 & \\
\hline $9: 20$ & $1: 26$ & 61 & 90 & 494 & 1991 & 364 & 261.3 & \\
\hline $9: 22$ & $1: 28$ & 61 & 89 & 498 & 1988 & 381 & 273.5 & \\
\hline $9: 24$ & $1: 30$ & 60 & 90 & 494 & 1981 & 361 & 259.1 & \\
\hline
\end{tabular}


MATR_4.XLS

\begin{tabular}{|c|c|c|c|c|c|c|c|c|}
\hline $\begin{array}{l}\text { TIME OF } \\
\text { DAY }\end{array}$ & $\begin{array}{c}\text { ELAPSED } \\
\text { TIME }\end{array}$ & $\begin{array}{c}\text { FEED } \\
\text { PRESSURE }\end{array}$ & FEED TEMP & FEED FLOW & $\begin{array}{l}\text { ROTOR } \\
\text { SPEED }\end{array}$ & $\begin{array}{c}\text { PERMEATE } \\
\text { FLOW }\end{array}$ & \begin{tabular}{|c|} 
PERMEATE \\
FLUUX
\end{tabular} & \\
\hline $9: 26$ & $1: 32$ & 56 & 90 & 493 & 1990 & 372 & 267.0 & \\
\hline $9: 28$ & $1: 34$ & 56 & 91 & 494 & 1991. & 335 & 240.5 & \\
\hline $9: 30$ & $1: 36$ & 56 & 91 & 495 & 2000 & 333 & 239.0 & \\
\hline $9: 32$ & $1: 38$ & 57 & 90 & 492 & 1990 & 321 & 230.4 & \\
\hline $9: 34$ & $1: 40$ & 58 & 89 & 494 & 1988 & 349 & 250.5 & \\
\hline $9: 36$ & $1: 42$ & 57 & 89 & 495 & 1994 & 358 & 257.0 & \\
\hline $9: 38$ & $1: 44$ & 54 & 89 & 489 & 1996 & 374 & 268.5 & \\
\hline $9: 40$ & $1: 46$ & 55 & 89 & 494 & 1988 & 372 & 267.0 & \\
\hline $9: 42$ & $1: 48$ & 59 & 90 & 483 & 1987 & 395 & 283.5 & \\
\hline $9: 44$ & $1: 50$ & 60 & 91 & 482 & 1985 & 353 & 253.4 & \\
\hline $9: 46$ & $1: 52$ & 65 & 92 & 484 & 1996 & 335 & 240.5 & \\
\hline $9: 48$ & $1: 54$ & 60 & 91 & 480 & 1989 & 340 & 244.1 & \\
\hline $9: 50$ & $1: 56$ & 59 & 89 & 481 & 1998 & 350 & 251.2 & \\
\hline $9: 52$ & $1: 58$ & 65 & 89 & 484 & 1993 & 362 & 259.9 & \\
\hline $9: 54$ & $2: 00$ & 61 & 89 & 482 & 1994 & 375 & 269.2 & \\
\hline $9: 56$ & $2: 02$ & 60 & 90 & 481 & 1996 & 376 & 269.9 & \\
\hline $9: 58$ & 2:04 & 62 & 89 & 482 & 1986 & 388 & 278.5 & \\
\hline $10: 01$ & $2: 07$ & 60 & 90 & 482 & 1989 & 378 & 271.3 & \\
\hline $10: 03$ & $2: 09$ & 65 & 91 & 481 & 1993 & 348 & 249.8 & \\
\hline $10: 05$ & $2: 11$ & 61 & 91 & 481 & 1985 & 326 & 234.0 & \\
\hline $10: 07$ & $2: 13$ & 63 & 90 & 479 & 1979 & 324 & 232.6 & \\
\hline $10: 09$ & $2: 15$ & 62 & 90 & 482 & 1994 & 325 & 233.3 & \\
\hline $10: 11$ & $2: 17$ & 61 & 87 & 480 & 1988 & 361 & 259.1 & \\
\hline $10: 13$ & $2: 19$ & 62 & 88 & 478 & 1982 & 370 & 265.6 & \\
\hline $10: 15$ & $2: 21$ & 61 & 88 & 480 & 1992 & 370 & 265.6 & \\
\hline $10: 17$ & $2: 23$ & 61 & 90 & 480 & 1989 & 371 & 266.3 & \\
\hline $10: 19$ & $2: 25$ & 62 & 90 & 482 & 1992 & 379 & 272.1 & \\
\hline $10: 21$ & $2: 27$ & 61 & 91 & 480 & 1986 & 360 & 258.4 & \\
\hline $10: 23$ & $2: 29$ & 60 & 91 & 480 & 1994 & 330 & 236.9 & \\
\hline $10: 25$ & $2: 31$ & 65 & 91 & 482 & 1992 & 324 & 232.6 & \\
\hline $10: 27$ & $2: 33$ & 65 & 91 & 479 & 1986 & 324 & 232.6 & \\
\hline $10: 29$ & $2: 35$ & 64 & 90 & 479 & 1997 & 351 & 252.0 & \\
\hline $10: 31$ & $2: 37$ & 61 & 89 & 480 & 1988 & 349 & 250.5 & \\
\hline $10: 33$ & $2: 39$ & 60 & 88 & 482 & 1987 & 360 & 258.4 & \\
\hline $10: 35$ & $2: 41$ & 61 & 89 & 482 & 1987 & 371 & 266.3 & \\
\hline $10: 37$ & $2: 43$ & 61 & 89 & 482 & 1998 & 375 & 269.2 & \\
\hline $10: 39$ & $2: 45$ & 63 & 90 & 481 & 1988 & 381 & 273.5 & \\
\hline $10: 41$ & $2: 47$ & 65 & 91 & 476 & 1990 & 349 & 250.5 & \\
\hline $10: 43$ & $2: 49$ & 61 & 90 & 477 & 1990 & 322 & 231.1 & \\
\hline $10: 45$ & $2: 51$ & 59 & 89 & 478 & 1996 & 311 & 223.2 & \\
\hline $10: 47$ & $2: 53$ & 61 & 89 & 477 & 1983 & 340 & 244.1 & \\
\hline $10: 49$ & $2: 55$ & 61 & 89 & 479 & 1977 & 366 & 262.7 & \\
\hline $10: 51$ & $2: 57$ & 61 & 89 & 479 & 1982 & 357 & 256.3 & \\
\hline $10: 53$ & $2: 59$ & 61 & 90 & 477 & 1991 & 378 & 271.3 & \\
\hline $10: 55$ & $3: 01$ & 63 & 90 & 476 & 1985 & 329 & 236.2 & End Steady \\
\hline
\end{tabular}


MATR_4.XLS

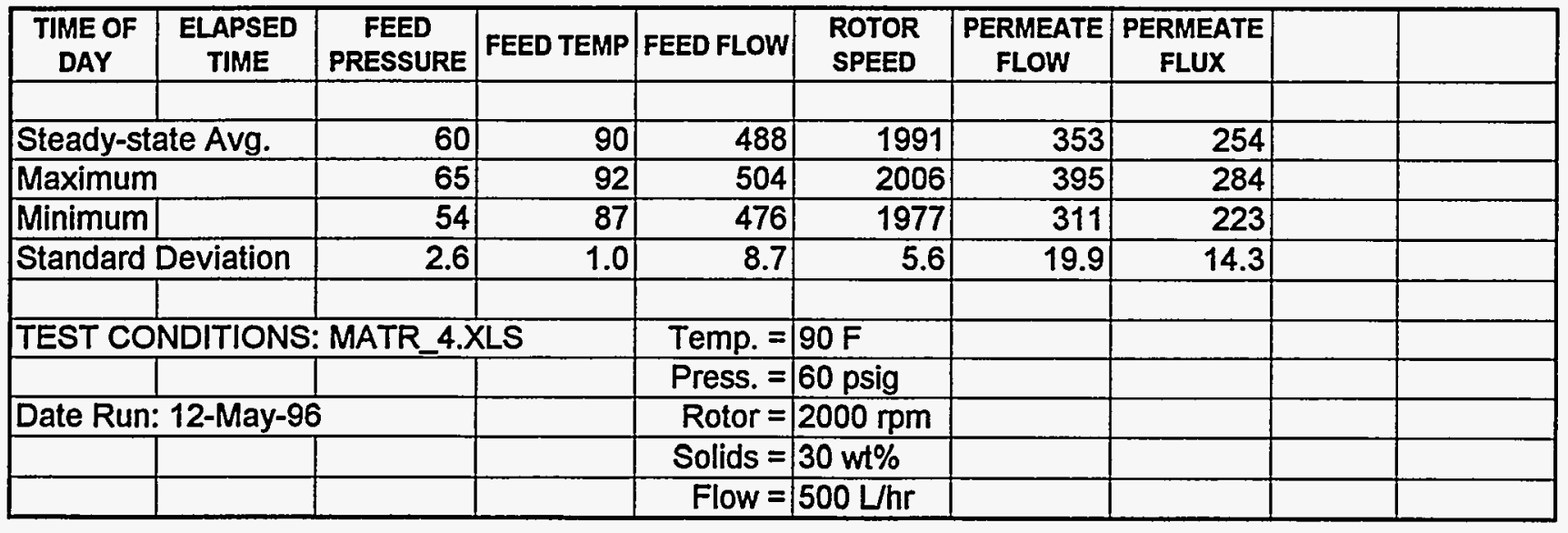


SpinTek II High Shear Rotary Membrane System

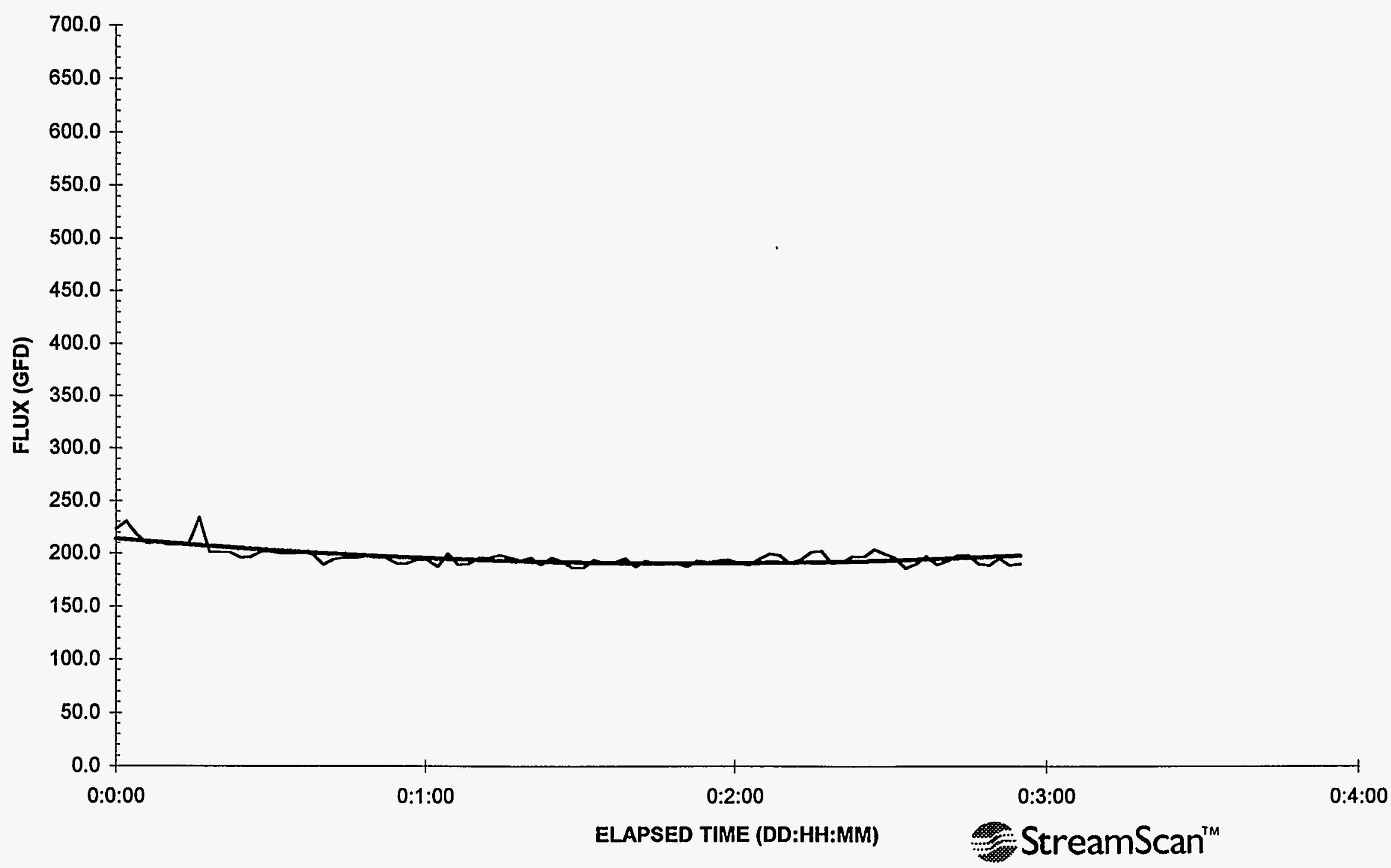




\begin{tabular}{|c|c|c|c|c|c|c|c|c|}
\hline $\begin{array}{c}\text { TIME OF } \\
\text { DAY }\end{array}$ & $\begin{array}{c}\text { ELAPSED } \\
\text { TIME }\end{array}$ & $\begin{array}{c}\text { FEED } \\
\text { PRESSURE }\end{array}$ & FEED TEMP & FEED FLOW & $\begin{array}{l}\text { ROTOR } \\
\text { SPEED }\end{array}$ & $\begin{array}{l}\text { PERMEATE } \\
\text { FLOW }\end{array}$ & $\begin{array}{l}\text { PERMEATE } \\
\text { FLUX }\end{array}$ & \\
\hline $0: 54$ & $0: 00$ & 50 & 91 & 482 & 1004 & 311 & 223.2 & \\
\hline $0: 56$ & $0: 02$ & 49 & 89 & 500 & 987 & 321 & 230.4 & \\
\hline $0: 58$ & $0: 04$ & 44 & 91 & 471 & 998 & 303 & 217.5 & \\
\hline $1: 00$ & $0: 06$ & 44 & 91 & 482 & 1000 & 291 & 208.9 & \\
\hline $1: 02$ & $0: 08$ & 44 & 90 & 445 & 990 & 293 & 210.3 & \\
\hline $1: 04$ & $0: 10$ & 46 & 90 & 470 & 990 & 290 & 208.2 & \\
\hline $1: 06$ & $0: 12$ & 49 & 91 & 464 & 989 & 290 & 208.2 & \\
\hline $1: 08$ & $0: 14$ & 47 & 90 & 485 & 992 & 292 & 209.6 & \\
\hline $1: 10$ & $0: 16$ & 54 & 90 & 489 & 989 & 326 & 234.0 & \\
\hline $1: 12$ & $0: 18$ & 45 & 90 & 484 & 995 & 280 & 201.0 & \\
\hline $1: 14$ & $0: 20$ & 46 & 90 & 446 & 987 & 280 & 201.0 & \\
\hline $1: 16$ & $0: 22$ & 43 & 91 & 470 & 990 & 280 & 201.0 & \\
\hline $1: 18$ & $0: 24$ & 46 & 91 & 486 & 984 & 273 & 196.0 & \\
\hline $1: 20$ & $0: 26$ & 43 & 89 & 478 & 990 & 274 & 196.7 & \\
\hline $1: 22$ & $0: 28$ & 47 & 90 & 482 & 985 & 281 & 201.7 & \\
\hline $1: 24$ & $0: 30$ & 46 & 89 & 472 & 984 & 281 & 201.7 & \\
\hline $1: 26$ & $0: 32$ & 44 & 90 & 484 & 990 & 278 & 199.6 & \\
\hline $1: 28$ & $0: 34$ & 45 & 90 & 463 & 990 & 278 & 199.6 & \\
\hline $1: 30$ & $0: 36$ & 48 & 90 & 492 & 990 & 280 & 201.0 & \\
\hline $1: 32$ & $0: 38$ & 44 & 91 & 516 & 983 & 277 & 198.8 & \\
\hline $1: 34$ & $0: 40$ & 43 & 90 & 493 & 990 & 264 & 189.5 & \\
\hline $1: 36$ & $0: 42$ & 46 & 89 & 483 & 988 & 271 & 194.5 & Begin Steady-state \\
\hline $1: 38$ & $0: 44$ & 43 & 90 & 484 & 987 & 273 & 196.0 & \\
\hline $1: 40$ & $0: 46$ & 48 & 89 & 458 & 989 & 272 & 195.2 & \\
\hline $1: 43$ & $0: 48$ & 46 & 90 & 464 & 996 & 275 & 197.4 & \\
\hline $1: 45$ & $0: 50$ & 44 & 90 & 467 & 991 & 273 & 196.0 & \\
\hline $1: 47$ & $0: 52$ & 44 & 90 & 485 & 985 & 273 & 196.0 & \\
\hline $1: 49$ & $0: 54$ & 43 & 90 & 493 & 986 & 266 & 190.9 & \\
\hline $1: 51$ & $0: 56$ & 43 & 90 & 470 & 983 & 265 & 190.2 & \\
\hline $1: 53$ & $0: 58$ & 48 & 89 & 487 & 988 & 270 & 193.8 & \\
\hline $1: 55$ & $1: 00$ & 47 & 90 & 469 & 1000 & 270 & 193.8 & \\
\hline $1: 57$ & $1: 02$ & 48 & 90 & 502 & 988 & 261 & 187.4 & \\
\hline $1: 59$ & $1: 04$ & 43 & 90 & 467 & 985 & 278 & 199.6 & \\
\hline $2: 01$ & $1: 06$ & 44 & 90 & 492 & 991 & 264 & 189.5 & \\
\hline $2: 03$ & $1: 08$ & 43 & 89 & 510 & 993 & 265 & 190.2 & \\
\hline $2: 05$ & $1: 10$ & 46 & 89 & 472 & 987 & 273 & 196.0 & \\
\hline $2: 07$ & $1: 12$ & 47 & 90 & 465 & 987 & 273 & 196.0 & \\
\hline $2: 09$ & $1: 14$ & 45 & 90 & 477 & 987 & 276 & 198.1 & \\
\hline $2: 11$ & $1: 16$ & 45 & 90 & 468 & 982 & 273 & 196.0 & \\
\hline $2: 13$ & $1: 18$ & 43 & 90 & 490 & 987 & 269 & 193.1 & \\
\hline $2: 15$ & $1: 20$ & 46 & 90 & 489 & 983 & 273 & 196.0 & \\
\hline $2: 17$ & $1: 22$ & 42 & 90 & 490 & 984 & 264 & 189.5 & \\
\hline $2: 19$ & $1: 24$ & 43 & 90 & 487 & 987 & 273 & 196.0 & \\
\hline $2: 21$ & $1: 26$ & 43 & 90 & 486 & 984 & 268 & 192.4 & \\
\hline $2: 23$ & $1: 28$ & 44 & 91 & 497 & 985 & 260 & 186.6 & \\
\hline
\end{tabular}


MATR_5.XLS

\begin{tabular}{|c|c|c|c|c|c|c|c|c|}
\hline $\begin{array}{c}\text { TIME OF } \\
\text { DAY }\end{array}$ & $\begin{array}{c}\text { ELAPSED } \\
\text { TIME }\end{array}$ & \begin{tabular}{|c|} 
FEED \\
PRESSURE \\
\end{tabular} & FEED TEMP & FEED FLOW & $\begin{array}{l}\text { ROTOR } \\
\text { SPEED }\end{array}$ & $\begin{array}{l}\text { PERMEATE } \\
\text { FLOW }\end{array}$ & $\begin{array}{c}\text { PERMEATE } \\
\text { FLUX }\end{array}$ & \\
\hline $2: 25$ & $1: 30$ & 45 & 90 & 486 & 983 & 260 & 186.6 & \\
\hline $2: 27$ & $1: 32$ & 43 & 90 & 459 & 992 & 270 & 193.8 & \\
\hline $2: 29$ & $1: 34$ & 44 & 90 & 466 & 983 & 266 & 190.9 & \\
\hline $2: 31$ & $1: 36$ & 46 & 90 & 496 & 986 & 267 & 191.7 & \\
\hline $2: 33$ & $1: 38$ & 46 & 90 & 480 & 991 & 272 & 195.2 & \\
\hline $2: 35$ & $1: 40$ & 45 & 90 & 505 & 987 & 261 & 187.4 & \\
\hline $2: 37$ & $1: 42$ & 42 & 90 & 483 & 983 & 269 & 193.1 & \\
\hline $2: 39$ & $1: 44$ & 42 & 89 & 450 & 985 & 266 & 190.9 & \\
\hline $2: 41$ & $1: 46$ & 43 & 90 & 460 & 987 & 265 & 190.2 & \\
\hline $2: 43$ & $1: 48$ & 42 & 90 & 479 & 987 & 266 & 190.9 & \\
\hline $2: 45$ & $1: 50$ & 45 & 89 & 497 & 984 & 261 & 187.4 & \\
\hline $2: 47$ & $1: 52$ & 46 & 89 & 469 & 985 & 269 & 193.1 & \\
\hline $2: 49$ & $1: 54$ & 42 & 90 & 471 & 983 & 267 & 191.7 & \\
\hline $2: 51$ & $1: 56$ & 43 & 90 & 469 & 985 & 269 & 193.1 & \\
\hline $2: 53$ & $1: 58$ & 42 & 90 & 487 & 984 & 270 & 193.8 & \\
\hline $2: 55$ & $2: 00$ & 42 & 90 & 446 & 985 & 266 & 190.9 & \\
\hline $2: 57$ & $2: 02$ & 42 & 90 & 477 & 986 & 263 & 188.8 & \\
\hline $2: 59$ & $2: 04$ & 46 & 90 & 474 & 982 & 271 & 194.5 & \\
\hline $3: 01$ & $2: 06$ & 44 & 90 & 499 & 983 & 278 & 199.6 & \\
\hline $3: 03$ & $2: 08$ & 48 & 90 & 494 & 983 & 276 & 198.1 & \\
\hline $3: 05$ & $2: 10$ & 45 & 91 & 486 & 983 & 266 & 190.9 & \\
\hline $3: 07$ & $2: 12$ & 44 & 90 & 446 & 983 & 270 & 193.8 & \\
\hline $3: 09$ & $2: 14$ & 43 & 89 & 502 & 982 & 281 & 201.7 & \\
\hline $3: 11$ & $2: 16$ & 43 & 89 & 473 & 984 & 282 & 202.4 & \\
\hline $3: 13$ & $2: 18$ & 47 & 89 & 484 & 983 & 266 & 190.9 & \\
\hline $3: 15$ & $2: 20$ & 48 & 90 & 481 & 986 & 267 & 191.7 & \\
\hline $3: 17$ & $2: 22$ & 48 & 91 & 487 & 983 & 275 & 197.4 & \\
\hline $3: 19$ & $2: 24$ & 47 & 90 & 460 & 988 & 274 & 196.7 & \\
\hline $3: 21$ & $2: 26$ & 48 & 90 & 474 & 982 & 284 & 203.9 & \\
\hline $3: 23$ & $2: 28$ & 49 & 90 & 477 & 979 & 278 & 199.6 & \\
\hline $3: 25$ & $2: 30$ & 47 & 91 & 490 & 986 & 273 & 196.0 & \\
\hline $3: 27$ & $2: 32$ & 43 & 90 & 459 & 984 & 259 & 185.9 & \\
\hline $3: 29$ & $2: 35$ & 47 & 89 & 494 & 982 & 265 & 190.2 & \\
\hline $3: 31$ & $2: 36$ & 45 & 89 & 480 & 995 & 275 & 197.4 & \\
\hline $3: 33$ & $2: 39$ & 43 & 89 & 491 & 983 & 264 & 189.5 & \\
\hline $3: 35$ & $2: 41$ & 45 & 89 & 493 & 982 & 269 & 193.1 & \\
\hline $3: 37$ & $2: 43$ & 47 & 89 & 477 & 983 & 276 & 198.1 & \\
\hline $3: 39$ & $2: 45$ & 47 & 90 & 496 & 983 & 276 & 198.1 & \\
\hline $3: 41$ & $2: 47$ & 46 & 90 & 464 & 983 & 265 & 190.2 & \\
\hline $3: 43$ & $2: 49$ & 48 & 90 & 464 & 983 & 263 & 188.8 & \\
\hline $3: 45$ & $2: 51$ & 47 & 89 & 494 & 981 & 272 & 195.2 & \\
\hline $3: 47$ & $2: 53$ & 43 & 90 & 481 & 982 & 263 & 188.8 & \\
\hline $3: 49$ & $2: 55$ & 48 & 90 & 487 & 986 & 265 & 190.2 & End Steady-state \\
\hline
\end{tabular}


MATR_5.XLS

\begin{tabular}{|c|c|c|c|c|c|c|c|c|c|}
\hline $\begin{array}{c}\text { TIME OF } \\
\text { DAY }\end{array}$ & $\begin{array}{c}\text { ELAPSED } \\
\text { TIME }\end{array}$ & $\begin{array}{c}\text { FEED } \\
\text { PRESSURE } \\
\end{array}$ & FEED TEMP & FEED FLOW & $\begin{array}{l}\text { ROTOR } \\
\text { SPEED }\end{array}$ & $\begin{array}{c}\begin{array}{c}\text { PERMEATE } \\
\text { FLOW }\end{array} \\
\end{array}$ & \begin{tabular}{|c|}
$\begin{array}{c}\text { PERMEATE } \\
\text { FLUX }\end{array}$ \\
\end{tabular} & & \\
\hline \multicolumn{2}{|c|}{ Steady-state Avg. } & 45 & 90 & 480 & 986 & 270 & 193 & & \\
\hline \multicolumn{2}{|c|}{ Maximum } & 49 & 91 & 510 & 1000 & 284 & 204 & & \\
\hline \multicolumn{2}{|c|}{ Minimum } & 42 & 89 & 446 & 979 & 259 & 186 & & \\
\hline \multicolumn{2}{|c|}{ Standard Deviation } & 2.1 & 0.5 & 14.4 & 3.8 & 5.6 & 4.0 & & \\
\hline & & & & & & & & & \\
\hline \multicolumn{4}{|c|}{ TEST CONDITIONS: MATR_5.XLS } & Temp. $=$ & $90 \mathrm{~F}$ & & & & \\
\hline & & & & Press. $=$ & $45 \mathrm{psig}$ & & & & \\
\hline \multicolumn{3}{|c|}{ Date Run: 13-May-96 } & & Rotor $=$ & $1000 \mathrm{rpm}$ & & & & \\
\hline & & & & Solids $=$ & $15 w \%$ & & & & \\
\hline & & & & Flow $=$ & $500 \mathrm{~L} / \mathrm{hr}$ & & & & \\
\hline
\end{tabular}


SpinTek II High Shear Rotary Membrane System

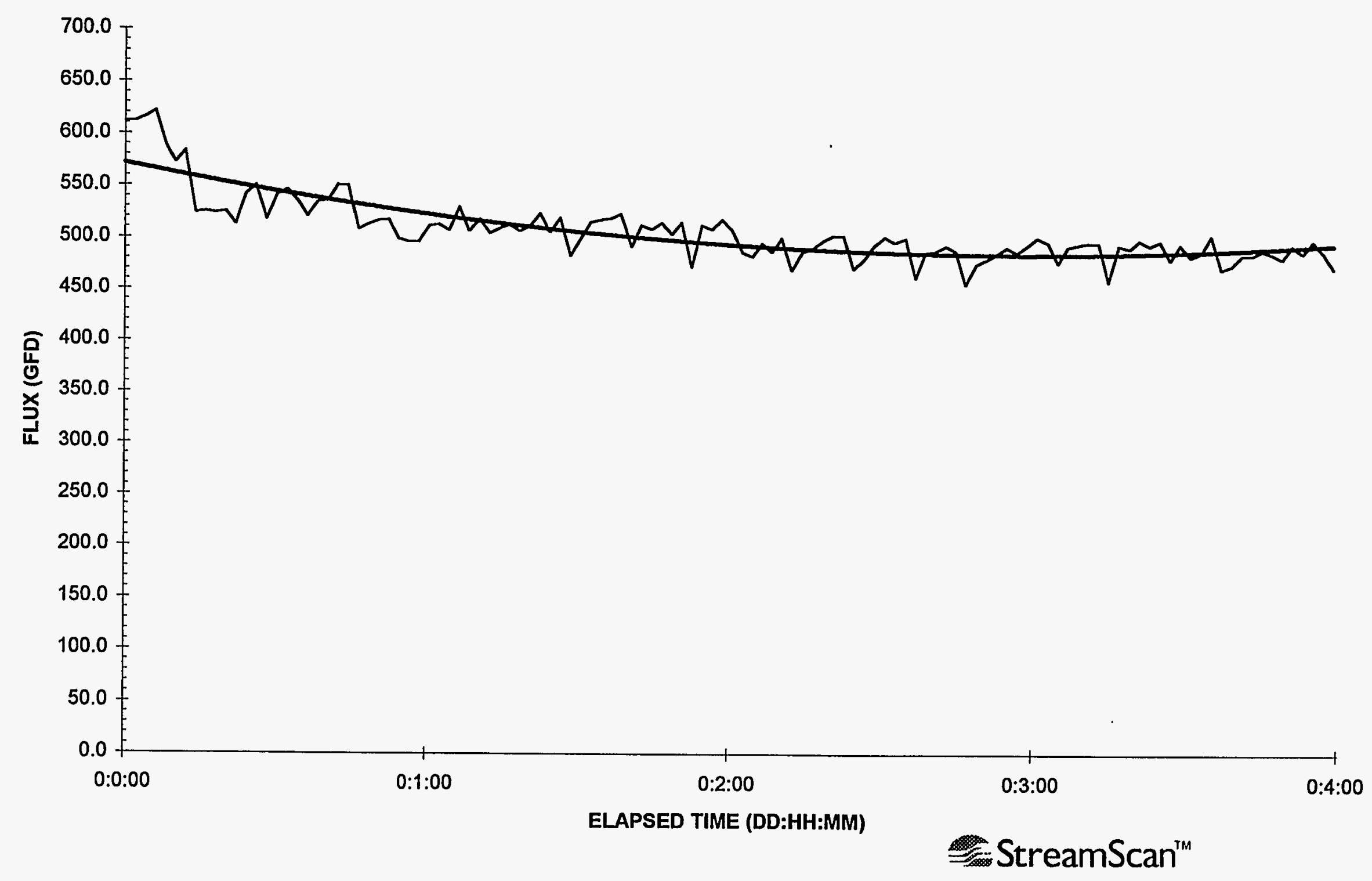




\begin{tabular}{|c|c|c|c|c|c|c|c|c|c|}
\hline $\begin{array}{l}\text { TIME OF } \\
\text { DAY }\end{array}$ & $\begin{array}{c}\text { ELAPSED } \\
\text { TIME }\end{array}$ & $\begin{array}{c}\text { FEED } \\
\text { PRESSURE } \\
\end{array}$ & FEED TEMP & FEED FLOW & $\begin{array}{l}\text { ROTOR } \\
\text { SPEED }\end{array}$ & $\begin{array}{c}\text { PERMEATE } \\
\text { FLOW }\end{array}$ & $\begin{array}{c}\text { PERMEATE } \\
\text { FLUX }\end{array}$ & & \\
\hline $15: 30$ & $0: 00$ & 81 & 89 & 381 & 1499 & 852 & 611.6 & & \\
\hline $15: 32$ & $0: 02$ & 75 & 89 & 391 & 1507 & 852 & 611.6 & & \\
\hline $15: 34$ & $0: 04$ & 78 & 89 & 382 & 1508 & 858 & 615.9 & & \\
\hline $15: 36$ & 0:06 & 83 & 89 & 382 & 1506 & 866 & 621.6 & & \\
\hline $15: 38$ & $0: 08$ & 82 & 90 & 390 & 1496 & 821 & 589.3 & & \\
\hline $15: 40$ & $0: 10$ & 79 & 90 & 406 & 1499 & 798 & 572.8 & & \\
\hline $15: 42$ & $0: 12$ & 82 & 89 & 394 & 1500 & 813 & 583.6 & & \\
\hline 15:44 & $0: 14$ & 67 & 90 & 446 & 1505 & 730 & 524.0 & & \\
\hline $15: 46$ & $0: 16$ & 71 & 91 & 412 & 1496 & 732 & 525.5 & & \\
\hline $15: 48$ & $0: 18$ & 73 & 91 & 443 & 1503 & 730 & 524.0 & & \\
\hline $15: 50$ & $0: 20$ & 72 & 91 & 410 & 1496 & 732 & 525.5 & & \\
\hline $15: 52$ & $0: 22$ & 73 & 90 & 448 & 1503 & 715 & 513.2 & & \\
\hline $15: 54$ & $0: 24$ & 70 & 91 & 407 & 1507 & 755 & 542.0 & & \\
\hline $15: 56$ & $0: 26$ & 75 & 89 & 430 & 1512 & 767 & 550.6 & & \\
\hline $15: 58$ & $0: 28$ & 75 & 90 & 423 & 1499 & 721 & 517.6 & & \\
\hline $16: 00$ & $0: 30$ & 77 & 90 & 403 & 1507 & 754 & 541.2 & & \\
\hline $16: 02$ & $0: 32$ & 73 & 90 & 436 & 1497 & 761 & 546.3 & & \\
\hline 16:04 & $0: 34$ & 79 & 91 & 416 & 1504 & 747 & 536.2 & & \\
\hline $16: 06$ & $0: 36$ & 75 & 91 & 414 & 1505 & 726 & 521.1 & & \\
\hline $16: 08$ & $0: 38$ & 79 & 88 & 446 & 1505 & 745 & 534.8 & & \\
\hline $16: 10$ & $0: 40$ & 78 & 89 & 417 & 1506 & 747 & 536.2 & & \\
\hline $16: 12$ & $0: 42$ & 78 & 89 & 421 & 1493 & 768 & 551.3 & & \\
\hline $16: 14$ & $0: 44$ & 79 & 90 & 448 & 1497 & 767 & 550.6 & & \\
\hline $16: 16$ & $0: 46$ & 75 & 91 & 424 & 1499 & 708 & 508.2 & & \\
\hline $16: 18$ & $0: 48$ & 74 & 91 & 458 & 1501 & 715 & 513.2 & & \\
\hline $16: 20$ & $0: 50$ & 73 & 90 & 429 & 1494 & 720 & 516.8 & & \\
\hline $16: 22$ & $0: 52$ & 75 & 91 & 456 & 1502 & 721 & 517.6 & & \\
\hline $16: 24$ & $0: 54$ & 73 & 91 & 454 & 1503 & 695 & 498.9 & & \\
\hline $16: 26$ & $0: 56$ & 70 & 89 & 421 & 1495 & 691 & 496.0 & & \\
\hline $16: 28$ & $0: 58$ & 75 & 89 & 445 & 1503 & 691 & 496.0 & & \\
\hline $16: 30$ & $1: 00$ & 75 & 90 & 447 & 1491 & 713 & 511.8 & & \\
\hline $16: 32$ & $1: 02$ & 69 & 89 & 417 & 1513 & 715 & 513.2 & & \\
\hline $16: 34$ & $1: 04$ & 72 & 90 & 457 & 1503 & 707 & 507.5 & & \\
\hline $16: 36$ & $1: 06$ & 75 & 90 & 442 & 1500 & 738 & 529.8 & & \\
\hline $16: 38$ & $1: 08$ & 73 & 90 & 428 & 1506 & 706 & 506.8 & & \\
\hline $16: 40$ & $1: 10$ & 73 & 91 & 461 & 1504 & 722 & 518.3 & & \\
\hline $16: 42$ & $1: 12$ & 72 & 89 & 421 & 1493 & 703 & 504.6 & & \\
\hline $16: 44$ & $1: 14$ & 71 & 89 & 441 & 1505 & 710 & 509.7 & & \\
\hline $16: 46$ & $1: 16$ & 78 & 90 & 460 & 1496 & 715 & 513.2 & & \\
\hline $16: 48$ & $1: 18$ & 72 & 89 & 428 & 1507 & 706 & 506.8 & & \\
\hline $16: 50$ & $1: 20$ & 77 & 91 & 455 & 1508 & 713 & 511.8 & & \\
\hline $16: 53$ & $1: 22$ & 75 & 90 & 448 & 1499 & 730 & 524.0 & & \\
\hline $16: 54$ & $1: 24$ & 75 & 90 & 429 & 1499 & 705 & 506.1 & & \\
\hline $16: 57$ & $1: 26$ & 72 & 91 & 464 & 1496 & 724 & 519.7 & & \\
\hline $16: 59$ & $1: 28$ & 77 & 89 & 434 & 1503 & 673 & 483.1 & & \\
\hline
\end{tabular}




\begin{tabular}{|c|c|c|c|c|c|c|c|c|}
\hline $\begin{array}{c}\text { TIME OF } \\
\text { DAY }\end{array}$ & $\begin{array}{c}\text { ELAPSED } \\
\text { TIME }\end{array}$ & $\begin{array}{c}\text { FEED } \\
\text { PRESSURE }\end{array}$ & FEED TEMP & FEED FLOW & $\begin{array}{l}\text { ROTOR } \\
\text { SPEED }\end{array}$ & \begin{tabular}{|c|} 
PERMEATE \\
FLOW
\end{tabular} & $\begin{array}{c}\text { PERMEATE } \\
\text { FLUX }\end{array}$ & \\
\hline $17: 01$ & $1: 30$ & 69 & 88 & 449 & 1499 & 695 & 498.9 & \\
\hline $17: 03$ & $1: 32$ & 77 & 89 & 455 & 1506 & 718 & 515.4 & \\
\hline $17: 05$ & $1: 34$ & 74 & 89 & 428 & 1497 & 721 & 517.6 & \\
\hline $17: 07$ & $1: 36$ & 73 & 90 & 456 & 1493 & 723 & 519.0 & \\
\hline $17: 09$ & $1: 38$ & 78 & 90 & 447 & 1496 & 729 & 523.3 & \\
\hline $17: 11$ & $1: 40$ & 75 & 90 & 447 & 1485 & 685 & 491.7 & \\
\hline $17: 13$ & $1: 42$ & 72 & 88 & 452 & 1501 & 714 & 512.5 & \\
\hline $17: 15$ & $1: 44$ & 73 & 89 & 440 & 1500 & 709 & 508.9 & \\
\hline $17: 17$ & $1: 46$ & 71 & 90 & 451 & 1502 & 717 & 514.7 & \\
\hline $17: 19$ & $1: 48$ & 74 & 89 & 427 & 1495 & 702 & 503.9 & \\
\hline $17: 21$ & $1: 50$ & 74 & 90 & 462 & 1496 & 718 & 515.4 & \\
\hline $17: 23$ & $1: 52$ & 73 & 90 & 424 & 1496 & 658 & 472.3 & \\
\hline $17: 25$ & $1: 54$ & 75 & 89 & 460 & 1505 & 714 & 512.5 & \\
\hline $17: 27$ & $1: 56$ & 75 & 89 & 423 & 1499 & 709 & 508.9 & \\
\hline $17: 29$ & $1: 58$ & 75 & 90 & 465 & 1496 & 722 & 518.3 & \\
\hline $17: 31$ & $2: 00$ & 76 & 90 & 424 & 1500 & 708 & 508.2 & \\
\hline $17: 33$ & $2: 02$ & 74 & 89 & 448 & 1498 & 678 & 486.7 & Begin Steady-state \\
\hline $17: 35$ & $2: 04$ & 72 & 89 & 440 & 1498 & 672 & 482.4 & \\
\hline $17: 37$ & $2: 06$ & 73 & 89 & 437 & 1497 & 690 & 495.3 & \\
\hline $17: 39$ & $2: 08$ & 74 & 89 & 425 & 1500 & 679 & 487.4 & \\
\hline $17: 41$ & $2: 10$ & 74 & 90 & 447 & 1499 & 697 & 500.3 & \\
\hline $17: 43$ & $2: 12$ & 74 & 90 & 424 & 1497 & 654 & 469.5 & \\
\hline $17: 45$ & $2: 15$ & 74 & 89 & 440 & 1497 & 679 & 487.4 & \\
\hline $17: 47$ & $2: 17$ & 71 & 89 & 454 & 1505 & 683 & 490.3 & \\
\hline $17: 49$ & $2: 19$ & 76 & 89 & 423 & 1500 & 693 & 497.5 & \\
\hline $17: 51$ & $2: 21$ & 74 & 89 & 444 & 1500 & 700 & 502.5 & \\
\hline $17: 53$ & $2: 23$ & 74 & 90 & 458 & 1491 & 700 & 502.5 & \\
\hline $17: 55$ & $2: 25$ & 71 & 90 & 427 & 1499 & 656 & 470.9 & \\
\hline $17: 57$ & $2: 27$ & 75 & 88 & 451 & 1500 & 669 & 480.2 & \\
\hline $17: 59$ & $2: 29$ & 71 & 88 & 467 & 1496 & 688 & 493.9 & \\
\hline $18: 01$ & $2: 31$ & 78 & 89 & 424 & 1506 & 699 & 501.8 & \\
\hline $18: 03$ & $2: 33$ & 73 & 90 & 436 & 1503 & 692 & 496.7 & \\
\hline $18: 05$ & $2: 35$ & 70 & 90 & 460 & 1502 & 697 & 500.3 & \\
\hline $18: 07$ & $2: 37$ & 72 & 89 & 435 & 1503 & 644 & 462.3 & \\
\hline $18: 09$ & $2: 39$ & 74 & 88 & 426 & 1497 & 678 & 486.7 & \\
\hline $18: 11$ & $2: 41$ & 72 & 88 & $\overline{443}$ & 1496 & 680 & 488.1 & \\
\hline $18: 13$ & $2: 43$ & 77 & 89 & 444 & 1503 & 687 & 493.1 & \\
\hline $18: 15$ & $2: 45$ & 74 & 90 & 426 & 1503 & 680 & 488.1 & \\
\hline $18: 17$ & $2: 47$ & 77 & 90 & 433 & 1504 & 635 & 455.8 & \\
\hline $18: 19$ & $2: 49$ & 69 & 90 & 448 & 1503 & 662 & 475.2 & \\
\hline $18: 21$ & $2: 51$ & 75 & 88 & 435 & 1497 & 669 & 480.2 & \\
\hline $18: 23$ & $2: 53$ & 72 & 88 & 459 & 1493 & 676 & 485.3 & \\
\hline $18: 25$ & $2: 55$ & 74 & 88 & 428 & 1494 & 685 & 491.7 & \\
\hline $18: 27$ & $2: 57$ & 78 & 89 & 460 & 1486 & 678 & 486.7 & \\
\hline $18: 29$ & $2: 59$ & 78 & 89 & 431 & 1497 & 688 & 493.9 & \\
\hline $18: 31$ & $3: 01$ & 75 & 90 & 469 & 1498 & 698 & 501.0 & \\
\hline $18: 33$ & $3: 03$ & 76 & 90 & 425 & 1503 & 692 & 496.7 & \\
\hline $18: 35$ & $3: 05$ & 73 & 90 & 461 & 1492 & 664 & 476.6 & \\
\hline $18: 37$ & $3: 07$ & 74 & 88 & 435 & 1496 & 686 & 492.4 & \\
\hline $18: 39$ & $3: 09$ & 70 & 89 & 445 & 1491 & 689 & 494.6 & \\
\hline
\end{tabular}


MATR_6.XLS

\begin{tabular}{|c|c|c|c|c|c|c|c|c|}
\hline $\begin{array}{l}\text { TIME OF } \\
\text { DAY }\end{array}$ & $\begin{array}{c}\text { ELAPSED } \\
\text { TIME }\end{array}$ & \begin{tabular}{|c|} 
FEED \\
PRESSURE \\
\end{tabular} & FEED TEMP & FEED FLOW & $\begin{array}{l}\text { ROTOR } \\
\text { SPEED }\end{array}$ & $\begin{array}{c}\text { PERMEATE } \\
\text { FLOW }\end{array}$ & $\begin{array}{c}\text { PERMEATE } \\
\text { FLUX } \\
\end{array}$ & \\
\hline $18: 41$ & $3: 11$ & 73 & 89 & 438 & 1500 & 692 & 496.7 & \\
\hline $18: 43$ & $3: 13$ & 75 & 90 & 439 & 1503 & 691 & 496.0 & \\
\hline $18: 45$ & $3: 15$ & 69 & 90 & 442 & 1501 & 639 & 458.7 & \\
\hline $18: 47$ & $3: 17$ & 78 & 89 & 451 & 1492 & 688 & 493.9 & \\
\hline $18: 49$ & $3: 19$ & 78 & 89 & 451 & 1496 & 684 & 491.0 & \\
\hline $18: 51$ & $3: 21$ & 72 & 89 & 424 & 1503 & 695 & 498.9 & \\
\hline $18: 53$ & $3: 23$ & 78 & 90 & 464 & 1495 & 688 & 493.9 & \\
\hline $18: 55$ & $3: 25$ & 75 & 90 & 429 & 1498 & 694 & 498.2 & \\
\hline $18: 57$ & $3: 27$ & 69 & 89 & 453 & 1500 & 669 & 480.2 & \\
\hline $18: 59$ & $3: 29$ & 69 & 89 & 423 & 1496 & 689 & 494.6 & \\
\hline 19:01 & $3: 31$ & 77 & 89 & 446 & 1499 & 673 & 483.1 & \\
\hline $19: 03$ & $3: 33$ & 72 & 89 & 446 & 1499 & 679 & 487.4 & \\
\hline $19: 05$ & $3: 35$ & 73 & 90 & 433 & 1495 & 701 & 503.2 & \\
\hline 19:07 & $3: 37$ & 75 & 91 & 457 & 1502 & 656 & 470.9 & \\
\hline $19: 09$ & $3: 39$ & 77 & 88 & 430 & 1494 & 661 & 474.5 & \\
\hline $19: 11$ & $3: 41$ & 69 & 88 & 439 & 1504 & 675 & 484.5 & \\
\hline $19: 13$ & $3: 43$ & 78 & 88 & 450 & 1499 & 675 & 484.5 & \\
\hline $19: 15$ & $3: 45$ & 73 & 89 & 412 & 1505 & 681 & 488.8 & \\
\hline $19: 17$ & $3: 47$ & 71 & 89 & 463 & 1504 & 677 & 486.0 & \\
\hline $19: 19$ & $3: 49$ & 74 & 90 & 422 & 1501 & 670 & 480.9 & \\
\hline $19: 21$ & $3: 51$ & 70 & 89 & 452 & 1489 & 688 & 493.9 & \\
\hline $19: 23$ & $3: 53$ & 75 & 89 & 452 & 1500 & 678 & 486.7 & \\
\hline $19: 25$ & $3: 55$ & 73 & 89 & 436 & 1504 & 694 & 498.2 & \\
\hline $19: 27$ & $3: 57$ & 74 & 89 & 456 & 1485 & 679 & 487.4 & \\
\hline $19: 29$ & $3: 59$ & 70 & 90 & 429 & 1496 & 657 & 471.6 & End Steady-state \\
\hline \multicolumn{2}{|c|}{ Steady-state Avg. } & 74 & 89 & 441 & 1498 & 679 & 488 & \\
\hline \multicolumn{2}{|l|}{ Maximum } & 78 & 91 & 469 & 1506 & 701 & 503 & \\
\hline Minimum & & 69 & 88 & 412 & 1485 & 635 & 456 & \\
\hline \multicolumn{2}{|c|}{ Standard Deviation } & 2.7 & 0.7 & 13.6 & 4.6 & 15.3 & 11.0 & \\
\hline \multicolumn{4}{|c|}{ TEST CONDITIONS: MATR_6.XLS } & Temp. $=$ & $90 \mathrm{~F}$ & & & \\
\hline-1 & & & & Press. $=$ & 75 psig & & & \\
\hline \multicolumn{3}{|c|}{ Date Run: 13-May-96 } & & Rotor $=$ & $1500 \mathrm{rpm}$ & & & \\
\hline & & & & Solids $=$ & $1 \mathrm{wt} \%$ & & & \\
\hline & & & & Flow $=1$ & $500 \mathrm{~L} / \mathrm{hr}$ & & & \\
\hline
\end{tabular}


SpinTek II High Shear Rotary Membrane System

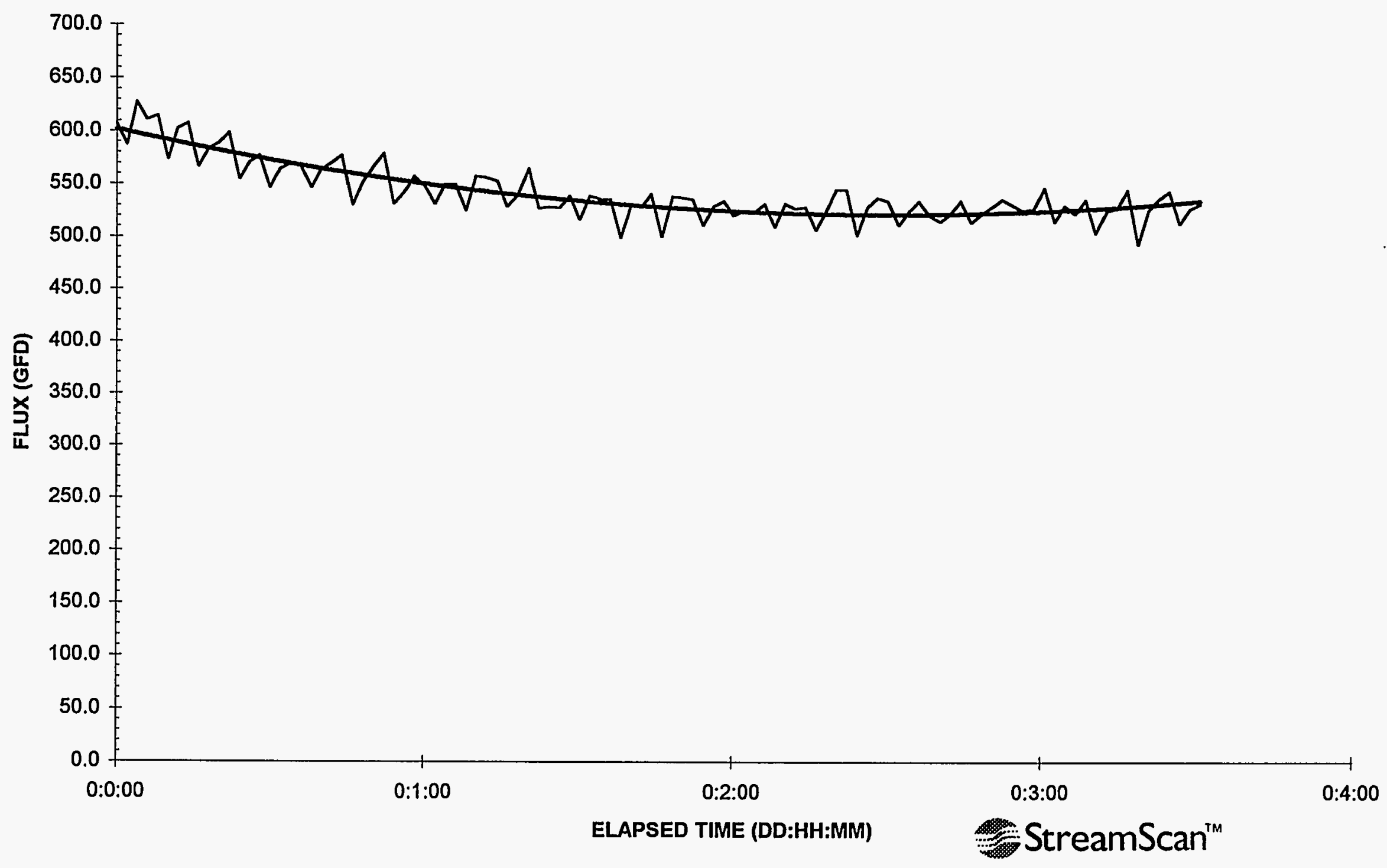




\begin{tabular}{|c|c|c|c|c|c|c|c|c|}
\hline $\begin{array}{c}\text { TIME OF } \\
\text { DAY }\end{array}$ & $\begin{array}{c}\text { ELAPSED } \\
\text { TIME }\end{array}$ & \begin{tabular}{c|} 
FEED \\
PRESSURE
\end{tabular} & FEED TEMP & FEED FLOW & $\begin{array}{l}\text { ROTOR } \\
\text { SPEED }\end{array}$ & $\begin{array}{l}\text { PERMEATE } \\
\text { FLOW }\end{array}$ & \begin{tabular}{|c|}
$\begin{array}{c}\text { PERMEATE } \\
\text { FLUX }\end{array}$ \\
\end{tabular} & \\
\hline $20: 24$ & $0: 00$ & 64 & 91 & 443 & 2013 & 846 & 607.3 & \\
\hline $20: 26$ & $0: 02$ & 58 & 89 & 442 & 2011 & 818 & 587.2 & \\
\hline $20: 28$ & $0: 03$ & 61 & 89 & 444 & 2010 & 874 & 627.4 & \\
\hline $20: 30$ & $0: 06$ & 63 & 90 & 452 & 2009 & 851 & 610.9 & \\
\hline $20: 32$ & $0: 08$ & 63 & 90 & 444 & 2010 & 856 & 614.5 & \\
\hline $20: 34$ & $0: 10$ & 59 & 90 & 438 & 2012 & 799 & 573.5 & \\
\hline $20: 36$ & $0: 12$ & 63 & 90 & 449 & 2010 & 839 & 602.3 & \\
\hline $20: 38$ & $0: 14$ & 60 & 89 & 450 & 2010 & 846 & 607.3 & \\
\hline $20: 40$ & $0: 16$ & 66 & 90 & 443 & 2019 & 789 & 566.4 & \\
\hline $20: 42$ & $0: 18$ & 65 & 89 & 442 & 2010 & 812 & 582.9 & \\
\hline $20: 44$ & $0: 20$ & 58 & 89 & 446 & 2010 & 820 & 588.6 & \\
\hline $20: 46$ & $0: 22$ & 65 & 90 & 441 & 2016 & 834 & 598.7 & \\
\hline $20: 48$ & $0: 24$ & 65 & 89 & 458 & 2009 & 773 & 554.9 & \\
\hline $20: 50$ & $0: 26$ & 57 & 89 & 439 & 2011 & 795 & 570.7 & \\
\hline $20: 52$ & $0: 28$ & 62 & 90 & 445 & 2010 & 804 & 577.1 & \\
\hline $20: 54$ & $0: 30$ & 60 & 91 & 452 & 2009 & 762 & 547.0 & \\
\hline $20: 56$ & $0: 32$ & 57 & 88 & 442 & 2010 & 786 & 564.2 & \\
\hline $20: 58$ & $0: 34$ & 59 & 89 & 445 & 2009 & 793 & 569.2 & \\
\hline $21: 00$ & $0: 36$ & 57 & 90 & 450 & 2015 & 790 & 567.1 & \\
\hline $21: 02$ & $0: 38$ & 63 & 90 & 450 & 2006 & 762 & 547.0 & \\
\hline $21: 04$ & $0: 40$ & 67 & 88 & 439 & 2013 & 785 & 563.5 & \\
\hline $21: 06$ & $0: 42$ & 59 & 89 & 439 & 2008 & 794 & 570.0 & \\
\hline $21: 08$ & $0: 44$ & 65 & 90 & 449 & 2013 & 804 & 577.1 & \\
\hline $21: 10$ & $0: 46$ & 66 & 90 & 433 & 2017 & 739 & 530.5 & \\
\hline $21: 12$ & $0: 48$ & 65 & 89 & 450 & 2009 & 769 & 552.0 & \\
\hline $21: 14$ & $0: 50$ & 60 & 89 & 450 & 2016 & 789 & 566.4 & \\
\hline $21: 16$ & $0: 52$ & 58 & 90 & 443 & 2006 & 806 & 578.6 & \\
\hline $21: 18$ & $0: 54$ & 58 & 90 & 455 & 2009 & 740 & 531.2 & \\
\hline $21: 20$ & $0: 56$ & 61 & 89 & 452 & 2005 & 755 & 542.0 & \\
\hline $21: 22$ & $0: 58$ & 61 & 89 & 432 & 2007 & 776 & 557.0 & \\
\hline $21: 24$ & $1: 00$ & 56 & 90 & 454 & 2007 & 764 & 548.4 & \\
\hline $21: 26$ & $1: 02$ & 63 & 89 & 446 & 2010 & 740 & 531.2 & \\
\hline $21: 28$ & $1: 04$ & 59 & 89 & 453 & 2010 & 766 & 549.9 & \\
\hline $21: 30$ & $1: 06$ & 67 & 90 & 446 & 2010 & 766 & 549.9 & \\
\hline $21: 32$ & $1: 08$ & 64 & 91 & 446 & 2010 & 732 & 525.5 & \\
\hline $21: 34$ & $1: 10$ & 57 & 89 & 446 & 2009 & 777 & 557.8 & \\
\hline $21: 36$ & $1: 12$ & 63 & 89 & 448 & 2005 & 775 & 556.3 & \\
\hline $21: 38$ & $1: 14$ & 63 & 90 & 446 & 2010 & 770 & 552.7 & \\
\hline $21: 40$ & $1: 16$ & 65 & 89 & 448 & 2010 & 737 & 529.0 & \\
\hline $21: 42$ & $1: 18$ & 63 & 89 & 446 & 2005 & 752 & 539.8 & \\
\hline $21: 44$ & $1: 20$ & 64 & 89 & 451 & 2011 & 787 & 564.9 & \\
\hline $21: 46$ & $1: 22$ & 66 & 90 & 441 & 2009 & 734 & 526.9 & Begin Steady-state \\
\hline $21: 48$ & $1: 24$ & 63 & 89 & 460 & 2007 & 736 & 528.3 & \\
\hline $21: 50$ & $1: 26$ & 60 & 89 & 450 & 2008 & 735 & 527.6 & \\
\hline $21: 52$ & $1: 28$ & 58 & 90 & 449 & 2009 & 751 & 539.1 & \\
\hline
\end{tabular}




\begin{tabular}{|c|c|c|c|c|c|c|c|c|c|}
\hline $\begin{array}{c}\text { TIME OF } \\
\text { DAY }\end{array}$ & $\begin{array}{c}\text { ELAPSED } \\
\text { TIME }\end{array}$ & $\begin{array}{c}\text { FEED } \\
\text { PRESSURE }\end{array}$ & FEED TEMP & FEED FLOW & $\begin{array}{l}\text { ROTOR } \\
\text { SPEED }\end{array}$ & $\begin{array}{c}\text { PERMEATE } \\
\text { FLOW }\end{array}$ & $\begin{array}{l}\text { PERMEATE } \\
\text { FLUX }\end{array}$ & & \\
\hline $21: 54$ & $1: 30$ & 60 & 91 & 449 & 2009 & 720 & 516.8 & & \\
\hline $21: 56$ & $1: 32$ & 62 & 88 & 444 & 2010 & 751 & 539.1 & & \\
\hline $21: 58$ & $1: 34$ & 65 & 89 & 452 & 2007 & 747 & 536.2 & & \\
\hline $22: 00$ & $1: 36$ & 62 & 90 & 454 & 2003 & 746 & 535.5 & & \\
\hline $22: 02$ & $1: 38$ & 61 & 90 & 446 & 2004 & 696 & 499.6 & & \\
\hline $22: 04$ & $1: 40$ & 62 & 89 & 457 & 2010 & 738 & 529.8 & & \\
\hline $22: 06$ & $1: 42$ & 62 & 89 & 453 & 2006 & 736 & 528.3 & & \\
\hline $22: 08$ & $1: 44$ & 61 & 90 & 448 & 2003 & 753 & 540.5 & & \\
\hline $22: 10$ & $1: 46$ & 59 & 89 & 448 & 2010 & 697 & 500.3 & & \\
\hline $22: 13$ & $1: 48$ & 60 & 88 & 454 & 2007 & 750 & 538.4 & & \\
\hline $22: 15$ & $1: 50$ & 62 & 89 & 452 & 2009 & 749 & 537.7 & & \\
\hline $22: 17$ & $1: 52$ & 62 & 90 & 452 & 2003 & 746 & 535.5 & & \\
\hline $22: 19$ & $1: 54$ & 62 & 89 & 456 & 2006 & 712 & 511.1 & & \\
\hline $22: 21$ & $1: 56$ & 58 & 89 & 455 & 2012 & 737 & 529.0 & & \\
\hline $22: 23$ & $1: 58$ & 56 & 89 & 439 & 2009 & 744 & 534.1 & & \\
\hline $22: 25$ & $2: 00$ & 64 & 90 & 450 & 2012 & 725 & 520.4 & & \\
\hline $22: 27$ & $2: 02$ & 62 & 88 & 442 & 2011 & 732 & 525.5 & & \\
\hline $22: 29$ & $2: 04$ & 57 & 89 & 451 & 2006 & 730 & 524.0 & & \\
\hline $22: 31$ & $2: 06$ & 57 & 89 & 448 & 2006 & 740 & 531.2 & & \\
\hline $22: 33$ & $2: 08$ & 63 & 90 & 441 & 2007 & 710 & 509.7 & & \\
\hline $22: 35$ & $2: 10$ & 62 & 89 & 459 & 2007 & 741 & 531.9 & & \\
\hline $22: 37$ & $2: 12$ & 58 & 89 & 436 & 2004 & 734 & 526.9 & & \\
\hline $22: 39$ & $2: 14$ & 62 & 90 & 456 & 2007 & 736 & 528.3 & & \\
\hline $22: 41$ & $2: 16$ & 62 & 90 & 450 & 2006 & 706 & 506.8 & & \\
\hline $22: 43$ & $2: 18$ & 63 & 89 & 455 & 2005 & 732 & 525.5 & & \\
\hline $22: 45$ & $2: 20$ & 65 & 89 & 453 & 2005 & 759 & 544.8 & & \\
\hline $22: 47$ & $2: 22$ & 63 & 90 & 459 & 2010 & 759 & 544.8 & & \\
\hline $22: 49$ & $2: 24$ & 58 & 90 & 448 & 2006 & 699 & 501.8 & & \\
\hline $22: 51$ & $2: 26$ & 65 & 88 & 451 & 2011 & 736 & 528.3 & & \\
\hline $22: 53$ & $2: 28$ & 63 & 89 & 454 & 2007 & 749 & 537.7 & & \\
\hline $22: 55$ & $2: 30$ & 63 & 90 & 454 & 2014 & 744 & 534.1 & & \\
\hline $22: 57$ & $2: 32$ & 63 & 89 & 442 & 2008 & 713 & 511.8 & & \\
\hline $22: 59$ & $2: 34$ & 58 & 89 & 448 & 2012 & 731 & 524.7 & & \\
\hline $23: 01$ & $2: 36$ & 57 & 89 & 454 & 2008 & 744 & 534.1 & & \\
\hline $23: 03$ & $2: 38$ & 59 & 91 & 455 & 2012 & 726 & 521.1 & & \\
\hline $23: 05$ & $2: 40$ & 63 & 89 & 446 & 2006 & 717 & 514.7 & & \\
\hline $23: 07$ & $2: 42$ & 61 & 89 & 447 & 2019 & 727 & 521.9 & & \\
\hline $23: 09$ & $2: 44$ & 65 & 90 & 454 & 2015 & 745 & 534.8 & & \\
\hline $23: 11$ & $2: 46$ & 65 & 90 & 455 & 2007 & 716 & 514.0 & & \\
\hline $23: 13$ & $2: 48$ & 64 & 88 & 445 & 2006 & 727 & 521.9 & & \\
\hline $23: 15$ & $2: 50$ & 59 & 89 & 444 & 2005 & 736 & 528.3 & & \\
\hline $23: 17$ & $2: 52$ & 61 & 89 & 453 & 2005 & 747 & 536.2 & & \\
\hline $23: 19$ & $2: 54$ & 63 & 90 & 438 & 2013 & 739 & 530.5 & & \\
\hline $23: 21$ & $2: 56$ & 58 & 89 & 440 & 2009 & 732 & 525.5 & & \\
\hline $23: 23$ & $2: 58$ & 63 & 89 & 458 & 2006 & 734 & 526.9 & & \\
\hline $23: 25$ & $3: 01$ & 64 & 89 & 450 & 2007 & 762 & 547.0 & & \\
\hline $23: 27$ & $3: 03$ & 63 & 90 & 454 & 2010 & 717 & 514.7 & & \\
\hline $23: 29$ & $3: 05$ & 56 & 88 & 458 & 2008 & 738 & 529.8 & & \\
\hline $23: 31$ & 3:07 & 61 & 89 & 449 & 2009 & 728 & 522.6 & & \\
\hline $23: 33$ & $3: 09$ & 58 & 90 & 451 & 2008 & 746 & 535.5 & & \\
\hline
\end{tabular}


MATR_7.XLS

\begin{tabular}{|c|c|c|c|c|c|c|c|c|}
\hline $\begin{array}{c}\text { TIME OF } \\
\text { DAY }\end{array}$ & $\begin{array}{c}\text { ELAPSED } \\
\text { TIME }\end{array}$ & \begin{tabular}{|c|} 
FEED \\
PRESSURE
\end{tabular} & FEED TEMP & FEED FLOW & $\begin{array}{c}\text { ROTOR } \\
\text { SPEED }\end{array}$ & $\begin{array}{l}\text { PERMEATE } \\
\text { FLOW }\end{array}$ & $\begin{array}{l}\text { PERMEATE } \\
\text { FLUX }\end{array}$ & \\
\hline $23: 35$ & $3: 11$ & 59 & 90 & 438 & 2007 & 702 & 503.9 & \\
\hline $23: 37$ & $3: 13$ & 63 & 88 & $\overline{454}$ & 2008 & 732 & 525.5 & \\
\hline $23: 39$ & $3: 15$ & 59 & 89 & 451 & 2008 & 735 & 527.6 & \\
\hline $23: 41$ & $3: 17$ & 63 & 90 & 454 & 2010 & 759 & 544.8 & \\
\hline $23: 43$ & $3: 19$ & 60 & 90 & 448 & 2006 & 687 & 493.1 & \\
\hline $23: 45$ & $3: 21$ & 61 & 88 & 453 & 1998 & 733 & 526.2 & \\
\hline $23: 47$ & $3: 23$ & 63 & 89 & 459 & 2006 & 748 & 536.9 & \\
\hline $23: 49$ & $3: 25$ & 63 & 90 & 453 & 2008 & 757 & 543.4 & \\
\hline $23: 51$ & $3: 27$ & 59 & 90 & 454 & 2006 & 715 & 513.2 & \\
\hline $23: 53$ & $3: 29$ & 59 & 89 & 456 & 2010 & 734 & 526.9 & \\
\hline $23: 55$ & $3: 31$ & 63 & 89 & 458 & 2008 & 741 & 531.9 & End Steady-state \\
\hline \multicolumn{2}{|c|}{ Steady-state Avg } & 61 & 89 & 451 & 2008 & 734 & 527 & \\
\hline \multicolumn{2}{|l|}{ Maximum } & 66 & 91 & 460 & 2019 & 762 & 547 & \\
\hline \multicolumn{2}{|l|}{ Minimum } & 56 & 88 & 436 & 1998 & 687 & 493 & \\
\hline \multicolumn{2}{|c|}{ Standard Deviation } & 2.5 & 0.7 & 5.8 & 3.2 & 16.7 & 12.0 & \\
\hline \multicolumn{4}{|c|}{ TEST CONDITIONS: MATR_7.XLS } & Temp. $=$ & $90 \mathrm{~F}$ & & & \\
\hline & & & & Press. $=$ & $60 \mathrm{psig}$ & & & \\
\hline \multicolumn{3}{|c|}{ Date Run: 13-May-96 } & & Rotor $=$ & $2000 \mathrm{rpm}$ & & & \\
\hline & & & & Solids $=$ & $1 w t \%$ & & & \\
\hline & & & & Flow $=$ & $500 \mathrm{~L} / \mathrm{hr}$ & & & \\
\hline
\end{tabular}




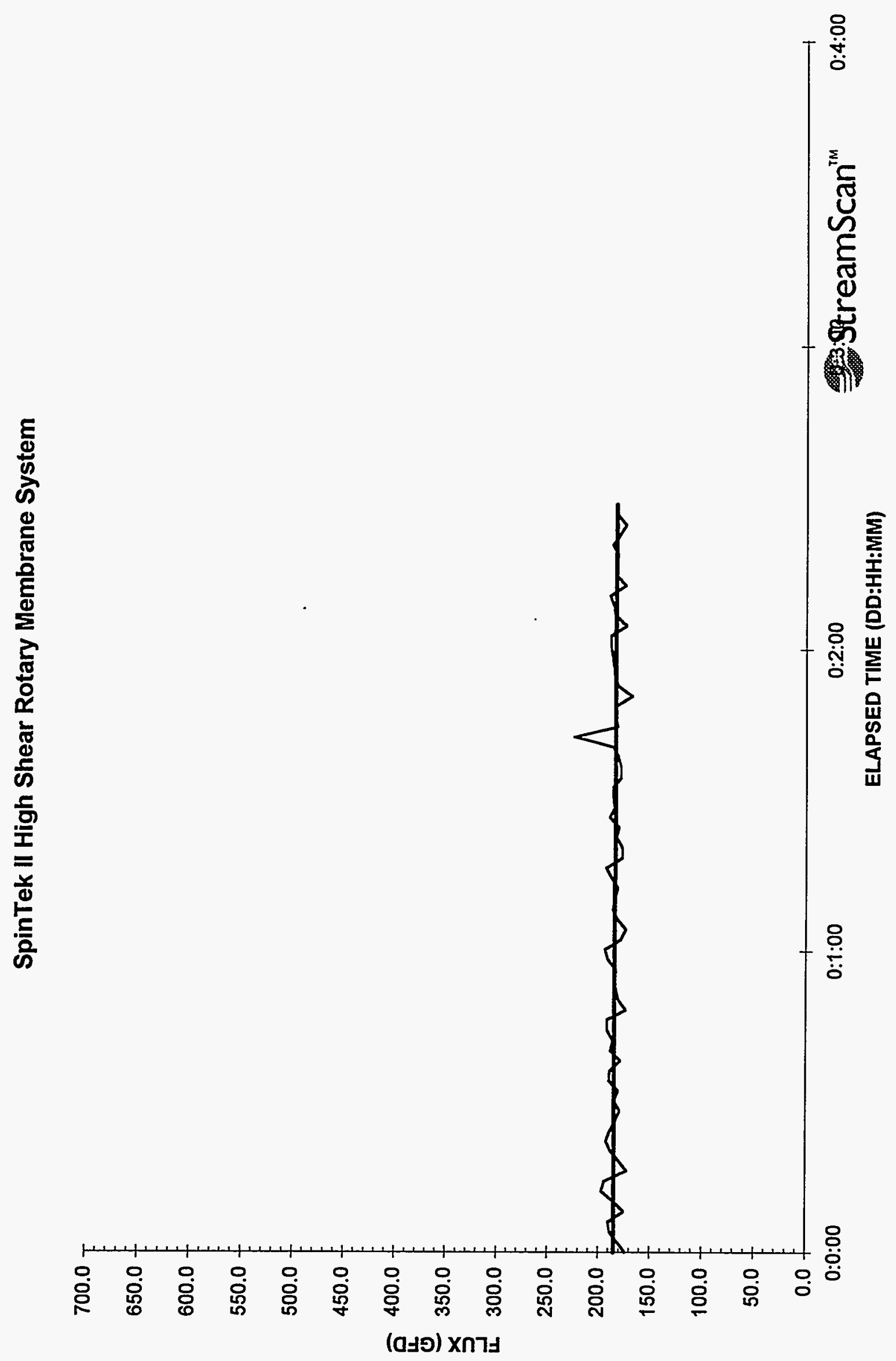




\begin{tabular}{|c|c|c|c|c|c|c|c|c|}
\hline $\begin{array}{c}\text { TIME OF } \\
\text { DAY }\end{array}$ & $\begin{array}{l}\text { ELAPSED } \\
\text { TIME }\end{array}$ & $\begin{array}{c}\text { FEED } \\
\text { PRESSURE }\end{array}$ & FEED TEMP & FEED FLOW & $\begin{array}{l}\text { ROTOR } \\
\text { SPEED }\end{array}$ & $\begin{array}{c}\text { PERMEATE } \\
\text { FLOW }\end{array}$ & $\begin{array}{c}\text { PERMEATE } \\
\text { FLUX }\end{array}$ & \\
\hline 1:06 & $0: 00$ & 45 & 90 & 491 & 1503 & 242 & 173.7 & \\
\hline $1: 08$ & $0: 01$ & 43 & 89 & 497 & 1500 & 252 & 180.9 & \\
\hline $1: 10$ & $0: 03$ & 44 & 89 & 487 & 1499 & 262 & 188.1 & \\
\hline $1: 12$ & $0: 05$ & 47 & 90 & 484 & 1501 & 265 & 190.2 & \\
\hline $1: 14$ & $0: 07$ & 43 & 90 & 483 & 1502 & 244 & 175.2 & \\
\hline $1: 16$ & $0: 09$ & 42 & 89 & 498 & 1501 & 257 & 184.5 & \\
\hline $1: 18$ & $0: 12$ & 46 & 89 & 487 & 1498 & 274 & 196.7 & \\
\hline $1: 20$ & $0: 14$ & 43 & 91 & 480 & 1506 & 270 & 193.8 & \\
\hline $1: 23$ & $0: 16$ & 41 & 90 & 482 & 1505 & 240 & 172.3 & \\
\hline $1: 25$ & $0: 18$ & 51 & 89 & 496 & 1501 & 251 & 180.2 & \\
\hline $1: 27$ & $0: 20$ & 51 & 89 & 497 & 1505 & 262 & 188.1 & \\
\hline $1: 29$ & $0: 22$ & 51 & 89 & 493 & 1508 & 268 & 192.4 & \\
\hline $1: 31$ & $0: 24$ & 40 & 90 & 485 & 1503 & 263 & 188.8 & \\
\hline $1: 33$ & $0: 26$ & 50 & 90 & 478 & 1504 & 256 & 183.8 & Begin Steady-state \\
\hline $1: 35$ & $0: 28$ & 53 & 89 & 484 & 1501 & 250 & 179.5 & \\
\hline $1: 37$ & $0: 30$ & 47 & 88 & 494 & 1504 & 258 & 185.2 & \\
\hline $1: 39$ & $0: 32$ & 46 & 88 & 495 & 1499 & 252 & 180.9 & \\
\hline $1: 41$ & $0: 34$ & 51 & 89 & 493 & 1502 & 264 & 189.5 & \\
\hline $1: 43$ & $0: 36$ & 51 & 90 & 496 & 1498 & 263 & 188.8 & \\
\hline $1: 45$ & $0: 38$ & 49 & 90 & 495 & 1506 & 249 & 178.7 & \\
\hline $1: 47$ & $0: 40$ & 51 & 90 & 490 & 1505 & 262 & 188.1 & \\
\hline $1: 49$ & $0: 42$ & 46 & 89 & 489 & 1503 & 259 & 185.9 & \\
\hline $1: 51$ & $0: 44$ & 52 & 89 & 491 & 1496 & 267 & 191.7 & \\
\hline $1: 53$ & $0: 46$ & 51 & 90 & 495 & 1496 & 267 & 191.7 & \\
\hline $1: 55$ & $0: 48$ & 45 & 90 & 494 & 1497 & 242 & 173.7 & \\
\hline $1: 57$ & $0: 50$ & 48 & 89 & 495 & 1507 & 252 & 180.9 & \\
\hline $1: 59$ & $0: 52$ & 43 & 88 & 493 & 1502 & 255 & 183.0 & \\
\hline $2: 01$ & $0: 54$ & 52 & 88 & 492 & 1505 & 257 & 184.5 & \\
\hline $2: 03$ & $0: 56$ & 45 & 89 & 485 & 1503 & 255 & 183.0 & \\
\hline $2: 05$ & $0: 58$ & 48 & 89 & 478 & 1495 & 266 & 190.9 & \\
\hline $2: 07$ & $1: 00$ & 47 & 90 & 478 & 1499 & 270 & 193.8 & \\
\hline $2: 09$ & $1: 02$ & 42 & 91 & 494 & 1501 & 249 & 178.7 & \\
\hline $2: 11$ & $1: 04$ & 43 & 90 & 494 & 1506 & 242 & 173.7 & \\
\hline $2: 13$ & $1: 06$ & 40 & 88 & 486 & 1502 & 254 & 182.3 & \\
\hline $2: 15$ & $1: 08$ & 52 & 88 & 475 & 1504 & 260 & 186.6 & \\
\hline $2: 17$ & $1: 10$ & 45 & 89 & 479 & 1495 & 257 & 184.5 & \\
\hline $2: 19$ & $1: 12$ & 42 & 89 & 492 & 1503 & 253 & 181.6 & \\
\hline $2: 21$ & $1: 14$ & 46 & 89 & 487 & 1502 & 261 & 187.4 & \\
\hline $2: 23$ & $1: 16$ & 50 & 90 & 482 & 1502 & 269 & 193.1 & \\
\hline $2: 25$ & $1: 18$ & 48 & 90 & 492 & 1490 & 248 & 178.0 & \\
\hline $2: 27$ & $1: 20$ & 52 & 91 & 484 & 1494 & 247 & 177.3 & \\
\hline $2: 29$ & $1: 22$ & 44 & 88 & 478 & 1497 & 255 & 183.0 & \\
\hline $2: 31$ & $1: 24$ & 48 & 88 & 491 & 1499 & 252 & 180.9 & \\
\hline $2: 33$ & $1: 26$ & 52 & 89 & 477 & 1502 & 264 & 189.5 & \\
\hline $2: 35$ & $1: 28$ & 51 & 89 & 492 & 1511 & 258 & 185.2 & \\
\hline
\end{tabular}


MATR_8.XLS

\begin{tabular}{|c|c|c|c|c|c|c|c|c|}
\hline $\begin{array}{c}\text { TIME OF } \\
\text { DAY }\end{array}$ & $\begin{array}{c}\text { ELAPSED } \\
\text { TIME } \\
\end{array}$ & $\begin{array}{c}\text { FEED } \\
\text { PRESSURE } \\
\end{array}$ & FEED TEMP & FEED FLOW & $\begin{array}{l}\text { ROTOR } \\
\text { SPEED } \\
\end{array}$ & $\begin{array}{c}\text { PERMEATE } \\
\text { FLOW }\end{array}$ & \begin{tabular}{|c|} 
PERMEATE \\
FLUX
\end{tabular} & \\
\hline $2: 37$ & $1: 30$ & 46 & 90 & 484 & 1497 & 260 & 186.6 & \\
\hline $2: 39$ & $1: 32$ & 47 & 90 & 482 & 1503 & 260 & 186.6 & \\
\hline $2: 41$ & $1: 34$ & 50 & 91 & 477 & 1503 & 249 & 178.7 & \\
\hline $2: 43$ & $1: 36$ & 40 & 89 & 477 & 1494 & 249 & 178.7 & \\
\hline $2: 45$ & $1: 38$ & 49 & 88 & 491 & 1494 & 253 & 181.6 & \\
\hline $2: 47$ & $1: 40$ & 52 & 88 & 478 & 1502 & 260 & 186.6 & \\
\hline $2: 49$ & $1: 42$ & 48 & 89 & 491 & 1499 & 313 & 224.7 & \\
\hline $2: 51$ & $1: 44$ & 44 & 89 & 477 & 1496 & 254 & 182.3 & \\
\hline $2: 53$ & $1: 46$ & 48 & 90 & 485 & 1505 & 257 & 184.5 & \\
\hline $2: 55$ & $1: 48$ & 48 & 90 & 487 & 1502 & 257 & 184.5 & \\
\hline $2: 57$ & $1: 50$ & 40 & 90 & 476 & 1503 & 234 & 168.0 & \\
\hline $2: 59$ & $1: 52$ & 50 & 89 & 490 & 1495 & 253 & 181.6 & \\
\hline $3: 01$ & $1: 54$ & 42 & 89 & 475 & 1500 & 258 & 185.2 & \\
\hline $3: 03$ & $1: 56$ & 42 & 89 & 491 & 1501 & 259 & 185.9 & \\
\hline $3: 05$ & $1: 58$ & 42 & 89 & 480 & 1500 & 261 & 187.4 & \\
\hline $3: 07$ & $2: 00$ & 42 & 90 & 474 & 1504 & 263 & 188.8 & \\
\hline $3: 09$ & $2: 02$ & 45 & 90 & 493 & 1499 & 263 & 188.8 & \\
\hline $3: 11$ & $2: 04$ & 49 & 90 & 473 & 1496 & 242 & 173.7 & \\
\hline $3: 13$ & $2: 06$ & 50 & 90 & 492 & 1503 & 257 & 184.5 & \\
\hline $3: 15$ & $2: 08$ & 44 & 89 & 482 & 1502 & 259 & 185.9 & \\
\hline $3: 17$ & $2: 10$ & 41 & 90 & 481 & 1503 & 264 & 189.5 & \\
\hline $3: 19$ & $2: 12$ & 42 & 90 & 483 & 1504 & 243 & 174.4 & \\
\hline $3: 21$ & $2: 14$ & 45 & 89 & 478 & 1503 & 256 & 183.8 & \\
\hline $3: 23$ & $2: 16$ & 43 & 89 & 489 & 1497 & 256 & 183.8 & \\
\hline $3: 25$ & $2: 18$ & 42 & 90 & 475 & 1503 & 254 & 182.3 & \\
\hline $3: 27$ & $2: 20$ & 43 & 90 & 482 & 1508 & 261 & 187.4 & \\
\hline $3: 29$ & $2: 22$ & 50 & 91 & 488 & 1496 & 252 & 180.9 & \\
\hline $3: 31$ & $2: 24$ & 43 & 90 & 473 & 1499 & 243 & 174.4 & \\
\hline $3: 33$ & $2: 26$ & 44 & 89 & 489 & 1503 & 256 & 183.8 & \\
\hline $3: 35$ & $2: 28$ & 46 & 89 & 485 & 1502 & 257 & 184.5 & End Steady-state \\
\hline \multicolumn{2}{|c|}{ Steady-state Avg. } & 47 & 89 & 185 & & 257 & & \\
\hline \multicolumn{2}{|l|}{ Maximum } & 53 & $\frac{89}{91}$ & $\frac{485}{496}$ & $\frac{1501}{1511}$ & $\frac{257}{313}$ & $\frac{184}{225}$ & \\
\hline Minimum & & 40 & 88 & $\frac{400}{473}$ & 1490 & $\frac{373}{234}$ & $\frac{225}{168}$ & \\
\hline \multicolumn{2}{|c|}{ Standard Deviation } & 3.7 & 0.8 & 7.0 & 4.0 & $\frac{234}{102}$ & $\frac{168}{72}$ & \\
\hline & & & & & & 70.2 & 7.3 & \\
\hline \multicolumn{9}{|c|}{ TEST CONDITIONS: MATR_8.XLS } \\
\hline & & & & Temp. $=$ & $90^{\circ} \mathrm{F}$ & & & \\
\hline \multicolumn{3}{|c|}{ Date Run: 14-May-96 } & & Press. $=$ & 45 psig & & & \\
\hline & & & & Rotor $=$ & $1500 \mathrm{rpm}$ & & & \\
\hline & & & & Solids $=$ & $30 w t \%$ & & & \\
\hline & & & & Flow $=$ & $500 \mathrm{l} / \mathrm{hr}$ & & & \\
\hline
\end{tabular}


SpinTek II High Shear Rotary Membrane System

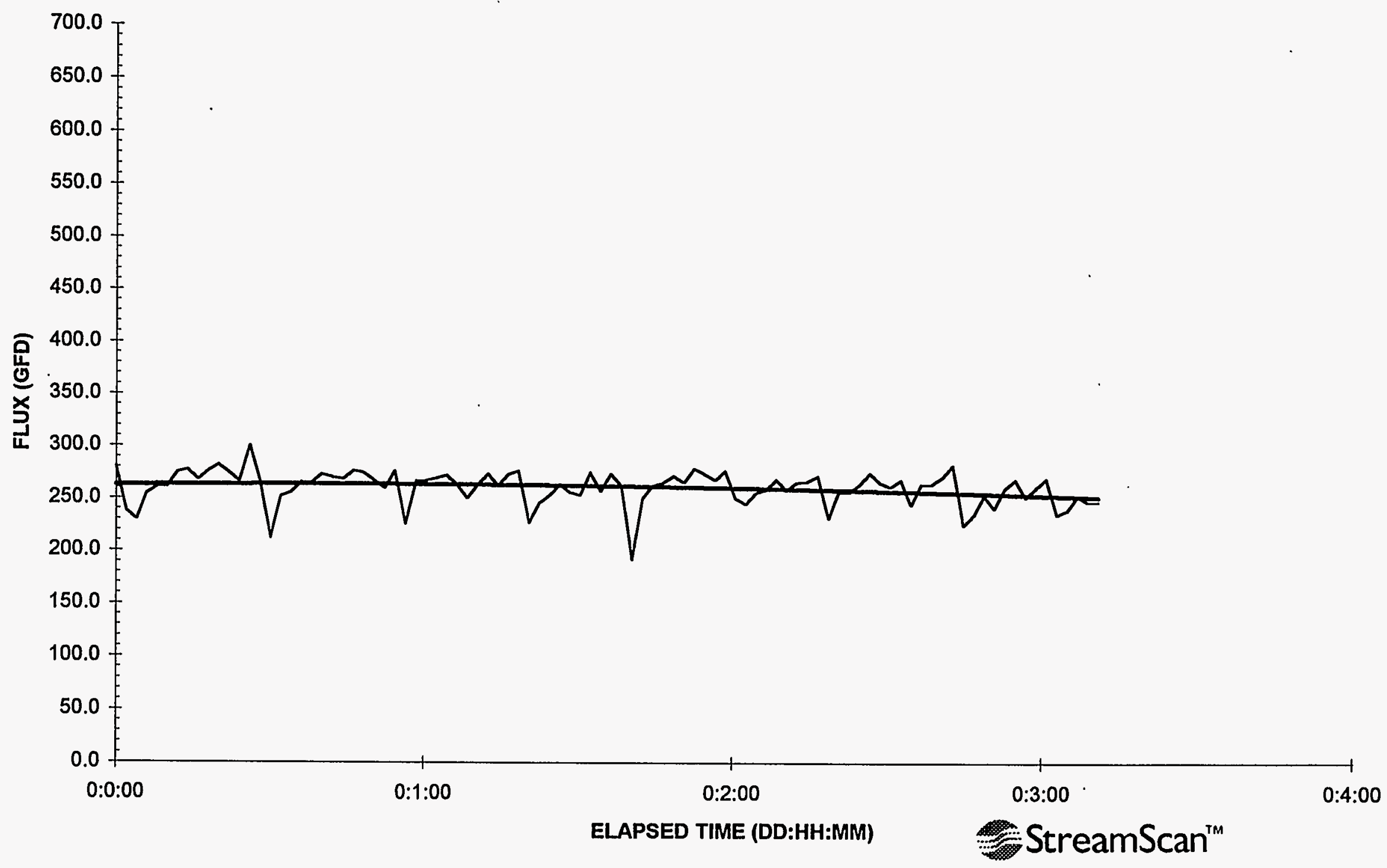




\begin{tabular}{|c|c|c|c|c|c|c|c|c|}
\hline $\begin{array}{c}\text { TIME OF } \\
\text { DAY }\end{array}$ & $\begin{array}{c}\text { ELAPSED } \\
\text { TIME }\end{array}$ & $\begin{array}{c}\text { FEED } \\
\text { PRESSURE }\end{array}$ & FEED TEMP & FEED FLOW & $\begin{array}{l}\text { ROTOR } \\
\text { SPEED }\end{array}$ & $\begin{array}{l}\text { PERMEATE } \\
\text { FLOW }\end{array}$ & $\begin{array}{l}\text { PERMEATE } \\
\text { FLUX }\end{array}$ & \\
\hline $5: 44$ & $0: 00$ & 65 & 119 & 511 & 1494 & 391 & 280.7 & \\
\hline $5: 46$ & $0: 02$ & 64 & 121 & 501 & 1499 & 331 & 237.6 & \\
\hline $5: 48$ & $0: 04$ & 66 & 119 & 499 & 1489 & 320 & 229.7 & \\
\hline $5: 50$ & $0: 06$ & 64 & 117 & 502 & 1499 & 354 & 254.1 & \\
\hline $5: 52$ & $0: 08$ & 65 & 116 & 501 & 1497 & 363 & 260.6 & \\
\hline $5: 54$ & $0: 10$ & 62 & 116 & 504 & 1502 & 364 & 261.3 & \\
\hline $5: 56$ & $0: 12$ & 64 & 116 & 506 & 1493 & 383 & 274.9 & \\
\hline $5: 58$ & $0: 14$ & 65 & 116 & 504 & 1485 & 386 & 277.1 & \\
\hline $6: 00$ & $0: 16$ & 63 & 117 & 503 & 1492 & 373 & 267.8 & \\
\hline $6: 02$ & $0: 18$ & 64 & 117 & 506 & 1496 & 385 & 276.4 & \\
\hline $6: 04$ & $0: 20$ & 66 & 118 & 505 & 1485 & 393 & 282.1 & \\
\hline $6: 06$ & $0: 22$ & 64 & 118 & 506 & 1503 & 382 & 274.2 & \\
\hline $6: 08$ & $0: 24$ & 55 & 118 & 505 & 1497 & 371 & 266.3 & \\
\hline $6: 10$ & $0: 26$ & 68 & 119 & 490 & 1502 & 418 & 300.1 & \\
\hline $6: 12$ & $0: 28$ & 64 & 120 & 492 & 1487 & 369 & 264.9 & \\
\hline $6: 14$ & $0: 30$ & 64 & 120 & 480 & 1491 & 295 & 211.8 & \\
\hline $6: 17$ & $0: 32$ & 61 & 117 & 481 & 1496 & 351 & 252.0 & \\
\hline $6: 19$ & $0: 34$ & 58 & 116 & 482 & 1490 & 356 & 255.5 & \\
\hline $6: 21$ & $0: 36$ & 61 & 117 & 493 & 1495 & 370 & 265.6 & \\
\hline $6: 23$ & $0: 38$ & 59 & 117 & 494 & 1492 & 369 & 264.9 & \\
\hline $6: 25$ & $0: 40$ & 62 & 117 & 497 & 1494 & 380 & 272.8 & \\
\hline $6: 27$ & $0: 42$ & 58 & 118 & 497 & 1486 & 376 & 269.9 & \\
\hline $6: 29$ & $0: 44$ & 63 & 119 & 497 & 1500 & 374 & 268.5 & \\
\hline $6: 31$ & $0: 46$ & 60 & 119 & 498 & 1495 & 385 & 276.4 & \\
\hline $6: 33$ & $0: 48$ & 64 & 120 & 499 & 1483 & 382 & 274.2 & \\
\hline $6: 35$ & $0: 50$ & 62 & 120 & 497 & 1486 & 372 & 267.0 & \\
\hline $6: 37$ & $0: 52$ & 61 & 120 & 487 & 1486 & 362 & 259.9 & \\
\hline $6: 39$ & $0: 54$ & 62 & 121 & 489 & 1483 & 384 & 275.6 & \\
\hline $6: 41$ & $0: 56$ & 63 & 121 & 485 & 1495 & 314 & 225.4 & \\
\hline $6: 43$ & $0: 58$ & 61 & 118 & 496 & 1482 & 371 & 266.3 & Begin Steady-state \\
\hline $6: 45$ & $1: 00$ & 62 & 117 & 495 & 1502 & 372 & 267.0 & \\
\hline $6: 47$ & $1: 02$ & 64 & 118 & 497 & 1503 & 375 & 269.2 & \\
\hline $6: 49$ & $1: 04$ & 63 & 118 & 497 & 1491 & 379 & 272.1 & \\
\hline $6: 51$ & $1: 06$ & 62 & 118 & 497 & 1500 & 366 & 262.7 & \\
\hline $6: 53$ & $1: 08$ & 65 & 119 & 499 & 1492 & 349 & 250.5 & \\
\hline $6: 55$ & $1: 10$ & 63 & 119 & 499 & 1496 & 366 & 262.7 & \\
\hline $6: 57$ & $1: 12$ & 59 & 120 & 499 & 1504 & 381 & 273.5 & \\
\hline $6: 59$ & $1: 14$ & 65 & 120 & 487 & 1492 & 365 & 262.0 & \\
\hline $7: 01$ & $1: 16$ & 64 & 121 & 487 & 1482 & 380 & 272.8 & \\
\hline $7: 03$ & $1: 18$ & 57 & 121 & 488 & 1491 & 385 & 276.4 & \\
\hline $7: 05$ & $1: 20$ & 58 & 120 & 492 & 1486 & 316 & 226.8 & \\
\hline $7: 07$ & $1: 22$ & 56 & 118 & 495 & 1496 & 342 & 245.5 & \\
\hline $7: 09$ & $1: 24$ & 61 & 117 & 493 & 1488 & 353 & 253.4 & \\
\hline $7: 11$ & $1: 26$ & 64 & 118 & 494 & 1485 & 367 & 263.4 & \\
\hline $7: 13$ & $1: 28$ & 62 & 119 & 494 & 1486 & 357 & 256.3 & \\
\hline
\end{tabular}


MATR_9.XLS

\begin{tabular}{|c|c|c|c|c|c|c|c|c|c|}
\hline $\begin{array}{l}\text { TIME OF } \\
\text { DAY }\end{array}$ & $\begin{array}{c}\text { ELAPSED } \\
\text { TIME }\end{array}$ & $\begin{array}{c}\text { FEED } \\
\text { PRESSURE }\end{array}$ & FEED TEMP & FEED FLOW & $\begin{array}{l}\text { ROTOR } \\
\text { SPEED }\end{array}$ & $\begin{array}{l}\text { PERMEATE } \\
\text { FLOW }\end{array}$ & $\begin{array}{l}\text { PERMEATE } \\
\text { FLUX }\end{array}$ & & \\
\hline $7: 15$ & $1: 30$ & 62 & 119 & 491 & 1494 & 353 & 253.4 & & \\
\hline $7: 17$ & $1: 32$ & 63 & 120 & 496 & 1480 & 383 & 274.9 & & \\
\hline $7: 19$ & $1: 34$ & 60 & 120 & 497 & 1489 & 358 & 257.0 & & \\
\hline $7: 21$ & $1: 36$ & 65 & 120 & 499 & 1503 & 381 & 273.5 & & \\
\hline $7: 23$ & $1: 38$ & 64 & 121 & 499 & 1494 & 365 & 262.0 & & \\
\hline $7: 25$ & $1: 40$ & 51 & 121 & 468 & 1481 & 267 & 191.7 & & \\
\hline $7: 27$ & $1: 42$ & 58 & 119 & 488 & 1490 & 349 & 250.5 & & \\
\hline $7: 29$ & $1: 44$ & 58 & 118 & 484 & 1490 & 365 & 262.0 & & \\
\hline $7: 31$ & $1: 46$ & 61 & 118 & 494 & 1491 & 369 & 264.9 & & \\
\hline $7: 33$ & $1: 48$ & 59 & 118 & 484 & 1493 & 378 & 271.3 & & \\
\hline $7: 35$ & $1: 50$ & 60 & 119 & 483 & 1503 & 370 & 265.6 & & \\
\hline $7: 37$ & $1: 52$ & 63 & 119 & 495 & 1489 & 388 & 278.5 & & \\
\hline $7: 39$ & $1: 54$ & 56 & 120 & 493 & 1497 & 381 & 273.5 & & \\
\hline $7: 41$ & $1: 56$ & 59 & 120 & 496 & 1494 & 373 & 267.8 & & \\
\hline $7: 43$ & $1: 58$ & 62 & 121 & 486 & 1493 & 385 & 276.4 & & \\
\hline $7: 45$ & $2: 00$ & 60 & 121 & 492 & 1494 & 349 & 250.5 & & \\
\hline $7: 47$ & $2: 02$ & 62 & 119 & 492 & 1485 & 341 & 244.8 & & \\
\hline $7: 49$ & $2: 04$ & 59 & 118 & 482 & 1489 & 356 & 255.5 & & \\
\hline $7: 51$ & $2: 06$ & 62 & 119 & 487 & 1489 & 360 & 258.4 & & \\
\hline $7: 53$ & $2: 08$ & 59 & 119 & 482 & 1485 & 374 & 268.5 & & \\
\hline $7: 55$ & $2: 10$ & 61 & 120 & 493 & 1496 & 359 & 257.7 & & \\
\hline $7: 57$ & $2: 12$ & 59 & 120 & 497 & 1484 & 370 & 265.6 & & \\
\hline $7: 59$ & $2: 14$ & 64 & 121 & 487 & 1495 & 371 & 266.3 & & \\
\hline $8: 01$ & $2: 16$ & 62 & 121 & 482 & 1489 & 378 & 271.3 & & \\
\hline $8: 03$ & $2: 18$ & 64 & 120 & 480 & 1489 & 322 & 231.1 & & \\
\hline $8: 05$ & $2: 20$ & 55 & 119 & 492 & 1495 & 357 & 256.3 & & \\
\hline $8: 07$ & $2: 22$ & 61 & 118 & 494 & 1497 & 357 & 256.3 & & \\
\hline $8: 09$ & $2: 24$ & 59 & 119 & 492 & 1493 & 368 & 264.2 & & \\
\hline $8: 11$ & $2: 26$ & 62 & 119 & 494 & 1493 & 382 & 274.2 & & \\
\hline $8: 13$ & $2: 28$ & 55 & 120 & 493 & 1489 & 370 & 265.6 & & \\
\hline $8: 15$ & $2: 30$ & 65 & 120 & 485 & 1482 & 364 & 261.3 & & \\
\hline $8: 17$ & $2: 32$ & 64 & 121 & 486 & 1483 & 373 & 267.8 & & \\
\hline $8: 19$ & $2: 34$ & 65 & 121 & 482 & 1496 & 340 & 244.1 & & \\
\hline $8: 21$ & $2: 36$ & 58 & 120 & 494 & 1484 & 367 & 263.4 & & \\
\hline $8: 23$ & $2: 39$ & 64 & 120 & 489 & 1489 & 368 & 264.2 & & \\
\hline $8: 25$ & $2: 41$ & 58 & 120 & 483 & 1485 & 377 & 270.6 & & \\
\hline $8: 27$ & $2: 43$ & 65 & 121 & 495 & 1483 & 393 & 282.1 & & \\
\hline $8: 29$ & $2: 45$ & 64 & 122 & 479 & 1482 & 313 & 224.7 & & \\
\hline $8: 31$ & $2: 47$ & 57 & 119 & 490 & 1496 & 328 & 235.4 & & \\
\hline $8: 33$ & $2: 49$ & 59 & 118 & 485 & 1496 & 352 & 252.7 & & \\
\hline $8: 35$ & $2: 51$ & 58 & 118 & 492 & 1504 & 336 & 241.2 & & \\
\hline $8: 37$ & $2: 53$ & 58 & 118 & 493 & 1484 & 362 & 259.9 & & \\
\hline $8: 39$ & $2: 55$ & 64 & 119 & 482 & 1497 & 374 & 268.5 & & \\
\hline $8: 41$ & $2: 57$ & 56 & 120 & 482 & 1482 & 350 & 251.2 & & \\
\hline $8: 43$ & $2: 59$ & 54 & 120 & 494 & 1489 & 363 & 260.6 & & \\
\hline $8: 45$ & $3: 01$ & 57 & 121 & 489 & 1496 & 375 & 269.2 & & \\
\hline $8: 47$ & $3: 03$ & 57 & 121 & 476 & 1489 & 327 & 234.7 & & \\
\hline $8: 49$ & $3: 05$ & 57 & 119 & 484 & 1489 & 333 & 239.0 & & \\
\hline $8: 51$ & $3: 07$ & 59 & 118 & 486 & 1484 & 352 & 252.7 & & \\
\hline $8: 53$ & $3: 09$ & 65 & 117 & 480 & 1491 & 344 & 246.9 & & \\
\hline
\end{tabular}


MATR_9.XLS

\begin{tabular}{|c|c|c|c|c|c|c|c|c|}
\hline $\begin{array}{l}\text { TIME OF } \\
\text { DAY }\end{array}$ & $\begin{array}{c}\text { ELAPSED } \\
\text { TIME }\end{array}$ & $\begin{array}{c}\text { FEED } \\
\text { PRESSURE }\end{array}$ & FEED TEMP & FEED FLOW & $\begin{array}{l}\text { ROTOR } \\
\text { SPEED }\end{array}$ & $\begin{array}{l}\text { PERMEATE } \\
\text { FLOW }\end{array}$ & $\begin{array}{l}\text { PERMEATE } \\
\text { FLUX }\end{array}$ & \\
\hline $8: 55$ & $3: 11$ & 58 & 118 & 477 & 1501 & 344 & 246.9 & End Steady-state \\
\hline \multirow{2}{*}{\multicolumn{2}{|c|}{$\frac{1}{\text { Steady-state Avg. }}$}} & & & & & & & \\
\hline & & 60 & 119 & 490 & 1491 & 360 & 259 & \\
\hline \multicolumn{2}{|c|}{ Maximum } & 65 & 122 & 499 & 1504 & 393 & 282 & \\
\hline Minimum & & 51 & 117 & 468 & 1480 & 267 & 192 & \\
\hline \multicolumn{2}{|c|}{ Standard Deviation } & 3.2 & 1.2 & 6.7 & 6.3 & 21.1 & 15.2 & \\
\hline & & & & & & & & \\
\hline \multicolumn{4}{|c|}{ TEST CONDITIONS: MATR_9.XLS } & Temp. $=$ & $120 \mathrm{~F}$ & & & \\
\hline & & & & Press. $=$ & $60 \mathrm{psig}$ & & & \\
\hline \multicolumn{3}{|c|}{ Date Run: 14-May-96 } & & Rotor $=$ & $1500 \mathrm{rpm}$ & & & \\
\hline & & & & Solids $=$ & $30 w t \%$ & & & \\
\hline & & & & Flow $=$ & $500 \mathrm{~L} / \mathrm{hr}$ & & & \\
\hline
\end{tabular}


SpinTek II High Shear Rotary Membrane System

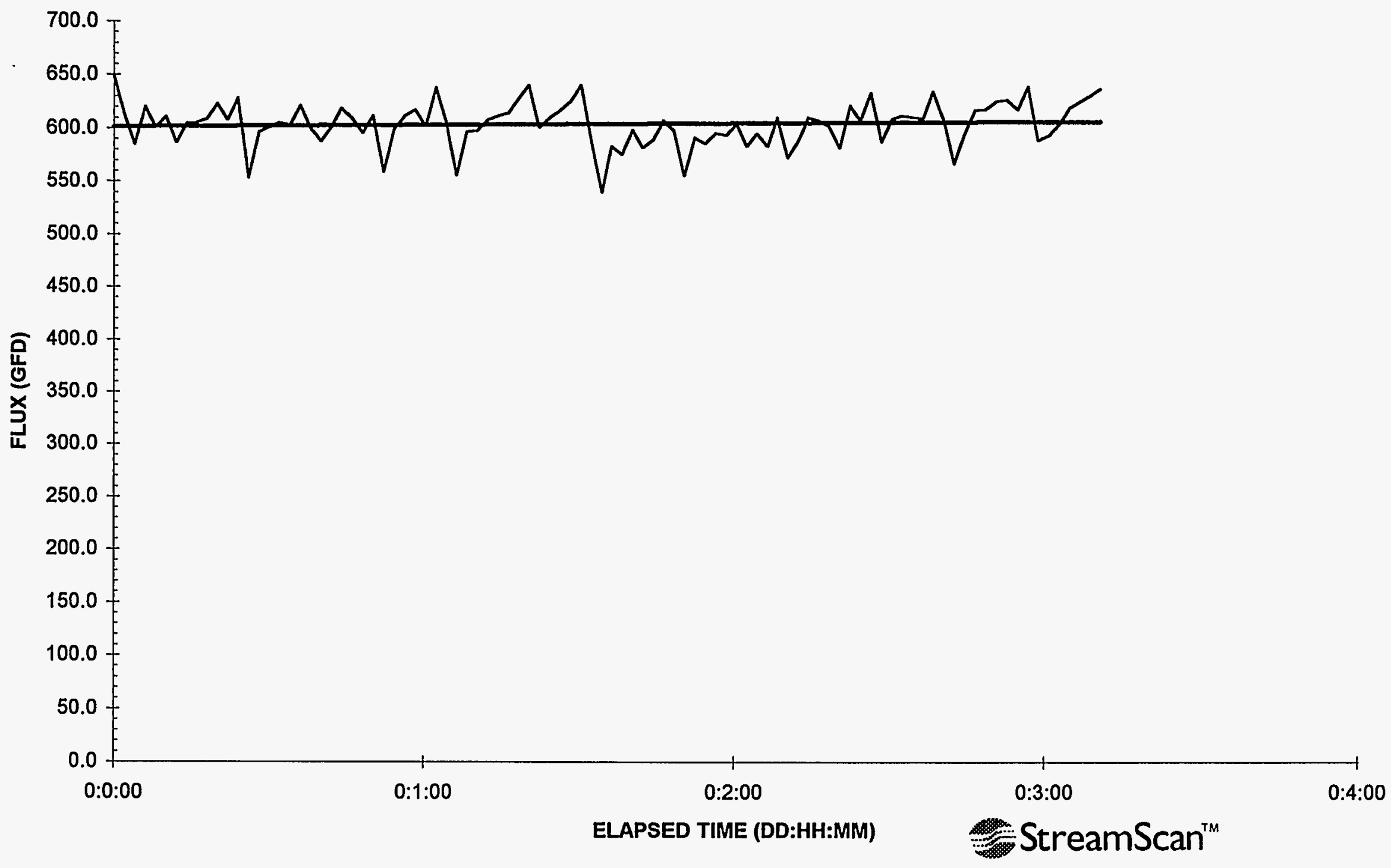




\begin{tabular}{|c|c|c|c|c|c|c|c|c|}
\hline $\begin{array}{c}\text { TIME OF } \\
\text { DAY }\end{array}$ & $\begin{array}{c}\text { ELAPSED } \\
\text { TIME }\end{array}$ & $\begin{array}{c}\text { FEED } \\
\text { PRESSURE }\end{array}$ & FEED TEMP & FEED FLOW & $\begin{array}{l}\text { ROTOR } \\
\text { SPEED }\end{array}$ & $\begin{array}{c}\text { PERMEATE } \\
\text { FLOW }\end{array}$ & $\begin{array}{l}\text { PERMEATE } \\
\text { FLUX }\end{array}$ & \\
\hline $12: 22$ & $0: 00$ & 69 & 120 & 464 & 1492 & 905 & 649.6 & \\
\hline $12: 24$ & $0: 02$ & 61 & 121 & 475 & 1502 & 855 & 613.7 & \\
\hline $12: 26$ & $0: 04$ & 65 & 122 & 480 & 1503 & 815 & 585.0 & \\
\hline $12: 28$ & $0: 06$ & 61 & 120 & 462 & 1504 & 864 & 620.2 & \\
\hline $12: 30$ & $0: 08$ & 64 & 120 & 476 & 1502 & 837 & 600.8 & \\
\hline $12: 32$ & $0: 10$ & 61 & 121 & 483 & 1504 & 851 & 610.9 & \\
\hline $12: 34$ & $0: 12$ & 62 & 118 & 464 & 1496 & 817 & 586.5 & \\
\hline $12: 36$ & $0: 14$ & 63 & 118 & 485 & 1506 & 842 & 604.4 & \\
\hline $12: 38$ & $0: 16$ & 63 & 118 & 460 & 1500 & 843 & 605.1 & \\
\hline $12: 40$ & $0: 18$ & 65 & 119 & 487 & 1495 & 848 & 608.7 & \\
\hline $12: 42$ & $0: 20$ & 60 & 120 & 469 & 1503 & 868 & 623.1 & \\
\hline $12: 44$ & $0: 22$ & 62 & 121 & 480 & 1494 & 847 & 608.0 & \\
\hline $12: 46$ & $0: 24$ & 64 & 121 & 487 & 1494 & 875 & 628.1 & \\
\hline $12: 48$ & $0: 26$ & 61 & 120 & 458 & 1504 & 771 & 553.4 & \\
\hline $12: 50$ & $0: 28$ & 62 & 117 & 480 & 1498 & 831 & 596.5 & \\
\hline $12: 52$ & $0: 30$ & 61 & 118 & 473 & 1495 & 837 & 600.8 & \\
\hline $12: 54$ & $0: 32$ & 64 & 119 & 463 & 1495 & 843 & 605.1 & \\
\hline $12: 56$ & $0: 34$ & 68 & 120 & 477 & 1500 & 840 & 603.0 & \\
\hline $12: 58$ & $0: 36$ & 64 & 120 & 484 & 1491 & 865 & 620.9 & \\
\hline $13: 00$ & $0: 38$ & 62 & 122 & 480 & 1501 & 836 & 600.1 & \\
\hline $13: 02$ & $0: 40$ & 64 & 119 & 475 & 1500 & 819 & 587.9 & \\
\hline $13: 04$ & $0: 42$ & 61 & 118 & 469 & 1499 & 838 & 601.5 & Begin Steady-state \\
\hline $13: 06$ & $0: 44$ & 61 & 119 & 462 & 1492 & 862 & 618.8 & \\
\hline $13: 08$ & $0: 46$ & 60 & 120 & 457 & 1494 & 849 & 609.4 & \\
\hline $13: 10$ & $0: 48$ & 63 & 120 & 459 & 1490 & 830 & 595.8 & \\
\hline $13: 12$ & $0: 50$ & 60 & 121 & 469 & 1503 & 852 & 611.6 & \\
\hline $13: 14$ & $0: 52$ & 63 & 121 & 463 & 1501 & 779 & 559.2 & \\
\hline $13: 16$ & $0: 54$ & 64 & 118 & 472 & 1499 & 835 & 599.4 & \\
\hline $13: 18$ & $0: 56$ & 62 & 119 & 463 & 1490 & 852 & 611.6 & \\
\hline $13: 20$ & $0: 58$ & 64 & 120 & 475 & 1503 & 860 & 617.3 & \\
\hline $13: 22$ & $1: 00$ & 60 & 120 & 485 & 1499 & 839 & 602.3 & \\
\hline $13: 24$ & $1: 02$ & 69 & 121 & 487 & 1491 & 889 & 638.1 & \\
\hline $13: 26$ & $1: 04$ & 60 & 121 & 469 & 1503 & 842 & 604.4 & \\
\hline $13: 28$ & $1: 06$ & 60 & 121 & 474 & 1490 & 774 & 555.6 & \\
\hline $13: 30$ & $1: 08$ & 63 & 118 & 489 & 1494 & 831 & 596.5 & \\
\hline $13: 32$ & $1: 10$ & 60 & 118 & 468 & 1500 & 833 & 598.0 & \\
\hline $13: 34$ & $1: 12$ & 66 & 119 & 478 & 1503 & 847 & 608.0 & \\
\hline $13: 36$ & $1: 14$ & 63 & 120 & 487 & 1502 & 852 & 611.6 & \\
\hline $13: 38$ & $1: 16$ & 60 & 120 & 469 & 1505 & 856 & 614.5 & \\
\hline $13: 40$ & $1: 18$ & 65 & 121 & 468 & 1499 & 875 & 628.1 & \\
\hline $13: 42$ & $1: 20$ & 67 & 122 & 498 & 1500 & 892 & 640.3 & \\
\hline $13: 44$ & $1: 22$ & 67 & 118 & 474 & 1502 & 837 & 600.8 & \\
\hline $13: 46$ & $1: 24$ & 67 & 118 & 465 & 1503 & 850 & 610.2 & \\
\hline $13: 48$ & $1: 26$ & 64 & 119 & 485 & 1500 & 859 & 616.6 & \\
\hline $13: 50$ & $1: 28$ & 64 & 119 & 485 & 1501 & 871 & 625.2 & \\
\hline
\end{tabular}


MATR_10.XLS

\begin{tabular}{|c|c|c|c|c|c|c|c|c|c|}
\hline $\begin{array}{c}\text { TIME OF } \\
\text { DAY }\end{array}$ & $\begin{array}{l}\text { ELAPSED } \\
\text { TIME }\end{array}$ & $\begin{array}{c}\text { FEED } \\
\text { PRESSURE }\end{array}$ & FEED TEMP & FEED FLOW & $\begin{array}{l}\text { ROTOR } \\
\text { SPEED }\end{array}$ & $\begin{array}{c}\text { PERMEATE } \\
\text { FLOW }\end{array}$ & $\begin{array}{l}\text { PERMEATE } \\
\text { FLUX }\end{array}$ & & \\
\hline $13: 52$ & $1: 30$ & 63 & 120 & 465 & 1499 & 892 & 640.3 & & \\
\hline $13: 54$ & $1: 32$ & 63 & 121 & 496 & 1509 & 819 & 587.9 & & \\
\hline $13: 56$ & $1: 34$ & 57 & 123 & 479 & 1498 & 752 & 539.8 & & \\
\hline $13: 58$ & $1: 36$ & 64 & 118 & 481 & 1501 & 812 & 582.9 & & \\
\hline $14: 00$ & $1: 38$ & 62 & 118 & 497 & 1502 & 802 & 575.7 & & \\
\hline $14: 02$ & $1: 40$ & 62 & 119 & 478 & 1503 & 834 & 598.7 & & \\
\hline $14: 04$ & $1: 42$ & 63 & 119 & 493 & 1497 & 811 & 582.2 & & \\
\hline 14:06 & $1: 44$ & 64 & 119 & 483 & 1497 & 821 & 589.3 & & \\
\hline $14: 08$ & $1: 46$ & 59 & 121 & 496 & 1494 & 846 & 607.3 & & \\
\hline $14: 10$ & $1: 48$ & 62 & 121 & 485 & 1505 & 834 & 598.7 & & \\
\hline $14: 12$ & $1: 50$ & 63 & 119 & 491 & 1502 & 774 & 555.6 & & \\
\hline $14: 14$ & $1: 52$ & 66 & 118 & 479 & 1493 & 824 & 591.5 & & \\
\hline $14: 16$ & $1: 54$ & 58 & 117 & 491 & 1502 & 816 & 585.7 & & \\
\hline $14: 18$ & $1: 56$ & 62 & 119 & 472 & 1503 & 830 & 595.8 & & \\
\hline $14: 20$ & $1: 58$ & 62 & 119 & 493 & 1506 & 827 & 593.6 & & \\
\hline $14: 22$ & $2: 00$ & 57 & 120 & 489 & 1503 & 842 & 604.4 & & \\
\hline $14: 24$ & $2: 02$ & 62 & 121 & 496 & 1498 & 813 & 583.6 & & \\
\hline $14: 26$ & $2: 04$ & 61 & 121 & 481 & 1493 & 829 & 595.1 & & \\
\hline $14: 28$ & $2: 06$ & 66 & 120 & 481 & 1496 & 813 & 583.6 & & \\
\hline $14: 30$ & $2: 08$ & 62 & 121 & 504 & 1499 & 850 & 610.2 & & \\
\hline $14: 32$ & $2: 10$ & 66 & 121 & 478 & 1497 & 798 & 572.8 & & \\
\hline $14: 34$ & $2: 12$ & 58 & 119 & 474 & 1502 & 819 & 587.9 & & \\
\hline $14: 36$ & $2: 14$ & 58 & 120 & 487 & 1502 & 850 & 610.2 & & \\
\hline $14: 39$ & $2: 16$ & 65 & 120 & 494 & 1502 & 846 & 607.3 & & \\
\hline $14: 41$ & $2: 18$ & 62 & 121 & 484 & 1503 & 839 & 602.3 & & \\
\hline $14: 43$ & $2: 20$ & 59 & 122 & 496 & 1496 & 811 & 582.2 & & \\
\hline $14: 45$ & $2: 22$ & 62 & 121 & 481 & 1501 & 866 & 621.6 & & \\
\hline $14: 47$ & $2: 24$ & 62 & 121 & 499 & 1496 & 847 & 608.0 & & \\
\hline $14: 49$ & $2: 26$ & 57 & 121 & 490 & 1496 & 882 & 633.1 & & \\
\hline $14: 51$ & $2: 28$ & 65 & 119 & 480 & 1502 & 819 & 587.9 & & \\
\hline $14: 53$ & $2: 31$ & 65 & 118 & 503 & 1496 & 848 & 608.7 & & \\
\hline $14: 55$ & $2: 33$ & 62 & 120 & 478 & 1504 & 853 & 612.3 & & \\
\hline $14: 57$ & $2: 35$ & 55 & 119 & 487 & 1502 & 851 & 610.9 & & \\
\hline $14: 59$ & $2: 37$ & 66 & 120 & 490 & 1502 & 849 & 609.4 & & \\
\hline $15: 01$ & $2: 39$ & 65 & 121 & 494 & 1503 & 884 & 634.6 & & \\
\hline $15: 03$ & $2: 41$ & 60 & 121 & 480 & 1499 & 848 & 608.7 & & \\
\hline $15: 05$ & $2: 43$ & 57 & 120 & 489 & 1502 & 790 & 567.1 & & \\
\hline $15: 07$ & $2: 45$ & 64 & 117 & 499 & 1504 & 828 & 594.4 & & \\
\hline $15: 09$ & $2: 47$ & 65 & 118 & 480 & 1499 & 860 & 617.3 & & \\
\hline $15: 11$ & $2: 49$ & 55 & 119 & 482 & 1499 & 861 & 618.1 & & \\
\hline $15: 13$ & $2: 51$ & 58 & 119 & 504 & 1505 & 872 & 625.9 & & \\
\hline $15: 15$ & $2: 53$ & 59 & 120 & 480 & 1504 & 874 & 627.4 & & \\
\hline $15: 17$ & $2: 55$ & 62 & 121 & 486 & 1496 & 861 & 618.1 & & \\
\hline $15: 19$ & $2: 57$ & 56 & 121 & 505 & 1504 & 891 & 639.6 & & \\
\hline $15: 21$ & $2: 59$ & 63 & 120 & 492 & 1496 & 821 & 589.3 & & \\
\hline $15: 23$ & $3: 01$ & 62 & 118 & 474 & 1503 & 827 & 593.6 & & \\
\hline $15: 25$ & $3: 03$ & 58 & 118 & 486 & 1502 & 842 & 604.4 & & \\
\hline $15: 27$ & $3: 05$ & 58 & 119 & 508 & 1503 & 863 & 619.5 & & \\
\hline $15: 29$ & $3: 07$ & 60 & 119 & 492 & 1503 & 871 & 625.2 & & \\
\hline $15: 31$ & $3: 09$ & 55 & 120 & 480 & 1494 & 879 & 631.0 & & \\
\hline
\end{tabular}


MATR_10.XLS

\begin{tabular}{|c|c|c|c|c|c|c|c|c|}
\hline $\begin{array}{c}\text { TIME OF } \\
\text { DAY }\end{array}$ & $\begin{array}{c}\text { ELAPSED } \\
\text { TIME }\end{array}$ & $\begin{array}{c}\text { FEED } \\
\text { PRESSURE }\end{array}$ & FEED TEMP & FEED FLOW & $\begin{array}{l}\text { ROTOR } \\
\text { SPEED }\end{array}$ & $\begin{array}{l}\text { PERMEATE } \\
\text { FLOW }\end{array}$ & $\begin{array}{c}\text { PERMEATE } \\
\text { FLUX }\end{array}$ & \\
\hline $15: 33$ & $3: 11$ & 56 & 120 & 473 & 1503 & 888 & 637.4 & End Steady-state \\
\hline \multicolumn{2}{|c|}{ Steady-state Avg. } & 62 & 120 & 483 & 1500 & 841 & 604 & \\
\hline \multicolumn{2}{|c|}{ Maximum } & 69 & 123 & 508 & 1509 & 892 & 640 & \\
\hline \multicolumn{2}{|l|}{ Minimum } & 55 & 117 & 457 & 1490 & 752 & 540 & \\
\hline \multicolumn{2}{|c|}{ Standard Deviation } & 3.2 & 1.3 & 11.8 & 4.2 & 29.0 & 20.8 & \\
\hline \multicolumn{4}{|c|}{ TEST CONDITIONS: MATR_10.XLS } & Temp. $=$ & $120 \mathrm{~F}$ & & & \\
\hline & & & & Press. $=$ & $60 \mathrm{psig}$ & & & \\
\hline \multicolumn{3}{|c|}{ Date Run: 14-May-96 } & & Rotor $=$ & $1500 \mathrm{rpm}$ & & & \\
\hline & & & & Solids = & $1 w t \%$ & & & \\
\hline & & & & Flow $=$ & $500 \mathrm{~L} / \mathrm{hr}$ & & & \\
\hline
\end{tabular}

Page 3 
SpinTek II High Shear Rotary Membrane System

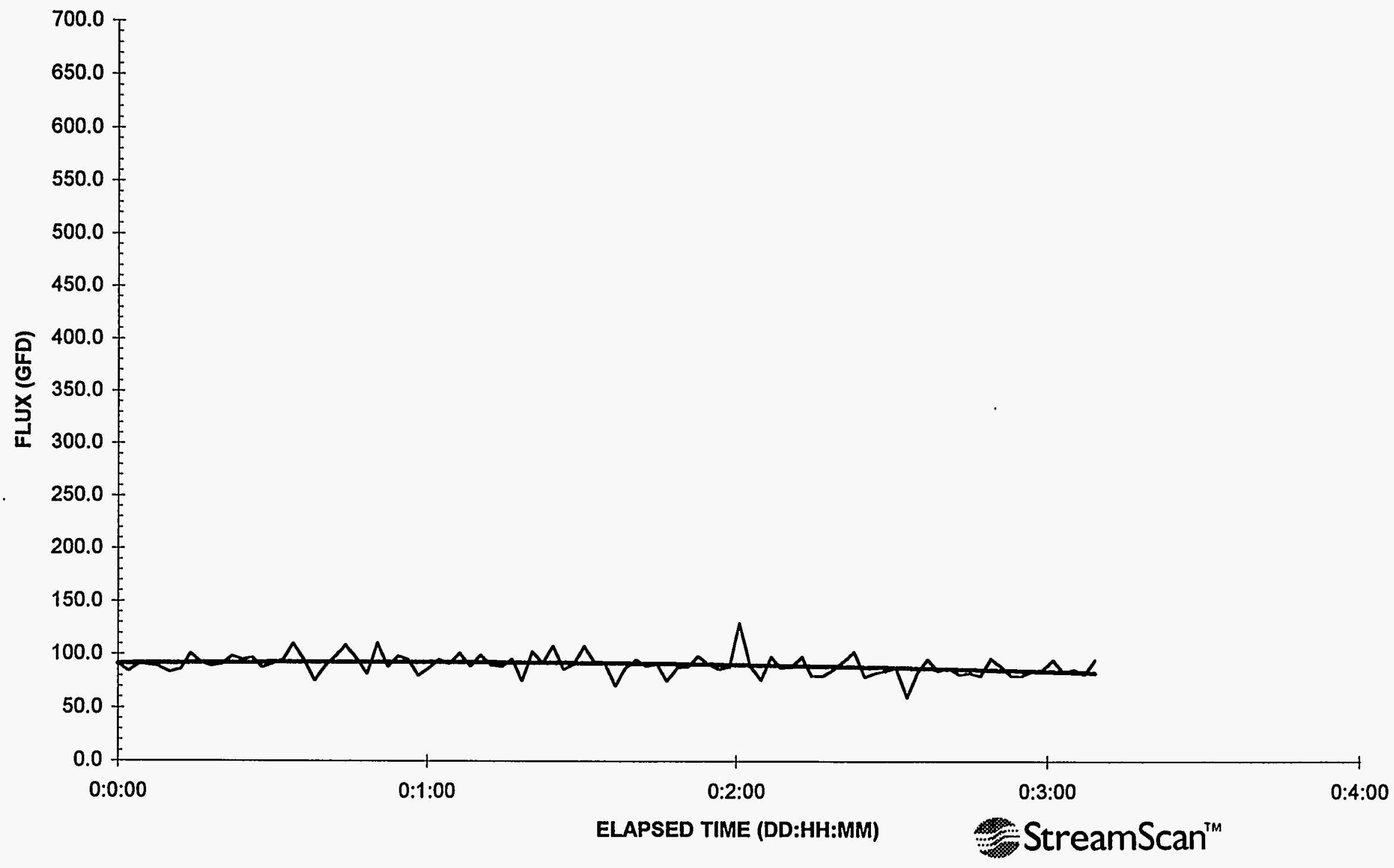




\begin{tabular}{|c|c|c|c|c|c|c|c|c|}
\hline $\begin{array}{c}\text { TIME OF } \\
\text { DAY }\end{array}$ & $\begin{array}{c}\text { ELAPSED } \\
\text { TIME }\end{array}$ & \begin{tabular}{c|} 
FEED \\
PRESSURE \\
\end{tabular} & FEED TEMP & FEED FLOW & $\begin{array}{l}\text { ROTOR } \\
\text { SPEED } \\
\end{array}$ & \begin{tabular}{|c|} 
PERMEATE \\
FLOW
\end{tabular} & $\begin{array}{c}\text { PERMEATE } \\
\text { FLUX } \\
\end{array}$ & \\
\hline $14: 39$ & $0: 00$ & 60 & 90 & 429 & 994 & 127 & 91.2 & \\
\hline $14: 41$ & $0: 02$ & 56 & 89 & 426 & 994 & 117 & 84.0 & \\
\hline $14: 43$ & $0: 04$ & 62 & 88 & 436 & 997 & 126 & 90.4 & \\
\hline $14: 45$ & $0: 06$ & 51 & 89 & 427 & 996 & 125 & 89.7 & \\
\hline $14: 47$ & $0: 08$ & 53 & 90 & 430 & 996 & 123 & 88.3 & \\
\hline $14: 49$ & $0: 10$ & 53 & 90 & 434 & 996 & 116 & 83.3 & \\
\hline $14: 51$ & $0: 12$ & 63 & 88 & 438 & 992 & 120 & 86.1 & \\
\hline $14: 53$ & $0: 14$ & 55 & 89 & 425 & 996 & 140 & 100.5 & \\
\hline $14: 55$ & $0: 16$ & 59 & 89 & 430 & 991 & 129 & 92.6 & \\
\hline $14: 57$ & $0: 18$ & 60 & 90 & 433 & 992 & 124 & 89.0 & Begin Steady-state \\
\hline $14: 59$ & $0: 20$ & 57 & 89 & 432 & 994 & 126 & 90.4 & \\
\hline $15: 01$ & $0: 22$ & 53 & 88 & 433 & 995 & 137 & 98.3 & \\
\hline $15: 03$ & $0: 24$ & 55 & 89 & 439 & 998 & 132 & 94.8 & \\
\hline $15: 05$ & $0: 26$ & 61 & 90 & 438 & 995 & 135 & 96.9 & \\
\hline $15: 07$ & $0: 28$ & 54 & 90 & 417 & 996 & 122 & 87.6 & \\
\hline $15: 09$ & $0: 30$ & 53 & 89 & 422 & 993 & 127 & 91.2 & \\
\hline $15: 11$ & $0: 32$ & 61 & 88 & 424 & 992 & 132 & 94.8 & \\
\hline $15: 13$ & $0: 34$ & 53 & 89 & 424 & 994 & 153 & 109.8 & \\
\hline $15: 15$ & $0: 36$ & 60 & 90 & 422 & 992 & 132 & 94.8 & \\
\hline $15: 17$ & $0: 38$ & 62 & 91 & 420 & 992 & 105 & 75.4 & \\
\hline $15: 19$ & $0: 40$ & 55 & 88 & 416 & 994 & 124 & 89.0 & \\
\hline $15: 21$ & $0: 42$ & 58 & 88 & 416 & 994 & 136 & 97.6 & \\
\hline $15: 23$ & $0: 44$ & 65 & 89 & 417 & 993 & 151 & 108.4 & \\
\hline $15: 25$ & $0: 46$ & 53 & 90 & 418 & 994 & 133 & 95.5 & \\
\hline $15: 27$ & $0: 48$ & 61 & 90 & 418 & 995 & 114 & 81.8 & \\
\hline $15: 29$ & $0: 50$ & 57 & 88 & 423 & 994 & 154 & 110.5 & \\
\hline $15: 31$ & $0: 52$ & 61 & 88 & 432 & 991 & 123 & 88.3 & \\
\hline $15: 33$ & $0: 54$ & 61 & 89 & 432 & 995 & 137 & 98.3 & \\
\hline $15: 35$ & $0: 56$ & 54 & 90 & 424 & 996 & 132 & 94.8 & \\
\hline $15: 37$ & $0: 58$ & 54 & 90 & 432 & 994 & 111 & 79.7 & \\
\hline $15: 39$ & $1: 00$ & 57 & 88 & 426 & 993 & 122 & 87.6 & \\
\hline $15: 41$ & $1: 02$ & 62 & 88 & 431 & 991 & 132 & 94.8 & \\
\hline $15: 43$ & $1: 04$ & 61 & 89 & 434 & 992 & 127 & 91.2 & \\
\hline $15: 45$ & $1: 06$ & 53 & 90 & 420 & 996 & 141 & 101.2 & \\
\hline $15: 47$ & $1: 08$ & 54 & 90 & 419 & 992 & 124 & 89.0 & \\
\hline $15: 49$ & $1: 10$ & 52 & 88 & 422 & 996 & 138 & 99.1 & \\
\hline $15: 51$ & $1: 12$ & 53 & 88 & 434 & 991 & 125 & 89.7 & \\
\hline $15: 53$ & $1: 14$ & 63 & 89 & 435 & 994 & 123 & 88.3 & \\
\hline $15: 55$ & $1: 16$ & 62 & 90 & 417 & 995 & 133 & 95.5 & \\
\hline $15: 57$ & $1: 18$ & 61 & 90 & 426 & 994 & 105 & 75.4 & \\
\hline $15: 59$ & $1: 20$ & 59 & 88 & 417 & 993 & 143 & 102.6 & \\
\hline $16: 01$ & $1: 22$ & 62 & 88 & 436 & 990 & 128 & 91.9 & \\
\hline $16: 03$ & $1: 24$ & 53 & 89 & 420 & 993 & 150 & 107.7 & \\
\hline $16: 05$ & $1: 26$ & 62 & 90 & 429 & 993 & 120 & 86.1 & \\
\hline $16: 07$ & $1: 28$ & 53 & 89 & 432 & 993 & 126 & 90.4 & \\
\hline
\end{tabular}




\begin{tabular}{|c|c|c|c|c|c|c|c|c|}
\hline $\begin{array}{l}\text { TIME OF } \\
\text { DAY }\end{array}$ & $\begin{array}{c}\text { ELAPSED } \\
\text { TIME }\end{array}$ & $\begin{array}{c}\text { FEED } \\
\text { PRESSURE } \\
\end{array}$ & FEED TEMP & FEED FLOW & $\begin{array}{l}\text { ROTOR } \\
\text { SPEED }\end{array}$ & \begin{tabular}{|c|} 
PERMEATE \\
FLOW
\end{tabular} & $\begin{array}{l}\text { PERMEATE } \\
\text { FLUX }\end{array}$ & \\
\hline $16: 09$ & $1: 30$ & 60 & 88 & 415 & 997 & 150 & 107.7 & \\
\hline $16: 11$ & $1: 32$ & 61 & 89 & 433 & 994 & 130 & 93.3 & \\
\hline $16: 13$ & $1: 34$ & 64 & 90 & 426 & 994 & 129 & 92.6 & \\
\hline $16: 15$ & $1: 36$ & 65 & 90 & 434 & 992 & 98 & 70.3 & \\
\hline $16: 17$ & $1: 38$ & 63 & 88 & 417 & 991 & 122 & 87.6 & \\
\hline $16: 19$ & $1: 40$ & 63 & 88 & 413 & 992 & 132 & 94.8 & \\
\hline $16: 21$ & $1: 42$ & 55 & 89 & 421 & 995 & 124 & 89.0 & \\
\hline $16: 23$ & $1: 44$ & 55 & 90 & 426 & 994 & 126 & 90.4 & \\
\hline $16: 25$ & $1: 46$ & 53 & 90 & 414 & 991 & 105 & 75.4 & \\
\hline $16: 27$ & $1: 48$ & 60 & 88 & 431 & 994 & 122 & 87.6 & \\
\hline $16: 29$ & $1: 50$ & 54 & 88 & 416 & 994 & 123 & 88.3 & \\
\hline $16: 31$ & $1: 52$ & 53 & 90 & 426 & 996 & 137 & 98.3 & \\
\hline $16: 33$ & $1: 54$ & 64 & 90 & 425 & 992 & 127 & 91.2 & \\
\hline $16: 35$ & $1: 56$ & 61 & 89 & 418 & 990 & 120 & 86.1 & \\
\hline $16: 37$ & $1: 58$ & 62 & 88 & 431 & 991 & 123 & 88.3 & \\
\hline $16: 39$ & $2: 00$ & 62 & 89 & 429 & 1004 & 180 & 129.2 & \\
\hline $16: 41$ & $2: 02$ & 63 & 90 & 415 & 991 & 125 & 89.7 & \\
\hline $16: 44$ & $2: 04$ & 57 & 90 & 430 & 994 & 106 & 76.1 & \\
\hline $16: 46$ & $2: 06$ & 61 & 88 & 419 & 991 & 136 & 97.6 & \\
\hline $16: 48$ & $2: 08$ & 54 & 88 & 421 & 992 & 122 & 87.6 & \\
\hline $16: 50$ & $2: 10$ & 58 & 89 & 424 & 991 & 123 & 88.3 & \\
\hline $16: 52$ & $2: 12$ & 61 & 90 & 431 & 994 & 136 & 97.6 & \\
\hline $16: 54$ & $2: 14$ & 53 & 90 & 414 & 997 & 111 & 79.7 & \\
\hline $16: 56$ & $2: 16$ & 57 & 88 & 415 & 992 & 111 & 79.7 & \\
\hline $16: 58$ & $2: 18$ & 62 & 89 & 415 & 994 & 120 & 86.1 & \\
\hline $17: 00$ & $2: 20$ & 52 & 90 & 427 & 994 & 131 & 94.0 & \\
\hline $17: 02$ & $2: 22$ & 53 & 90 & 413 & 991 & 143 & 102.6 & \\
\hline $17: 04$ & $2: 25$ & 64 & 90 & 415 & 990 & 110 & 79.0 & \\
\hline $17: 06$ & $2: 27$ & 64 & 88 & 415 & 991 & 115 & 82.6 & \\
\hline $17: 08$ & $2: 29$ & 57 & 88 & 413 & 990 & 118 & 84.7 & \\
\hline $17: 10$ & $2: 31$ & 59 & 89 & 426 & 997 & 122 & 87.6 & \\
\hline $17: 12$ & $2: 33$ & 55 & 90 & 433 & 990 & 83 & 59.6 & \\
\hline $17: 14$ & $2: 35$ & 62 & 90 & 429 & 990 & 115 & 82.6 & \\
\hline $17: 16$ & $2: 37$ & $64-$ & 89 & 426 & 990 & 133 & 95.5 & \\
\hline $17: 18$ & $2: 39$ & 60 & 88 & 426 & 990 & 118 & 84.7 & \\
\hline $17: 20$ & $2: 41$ & 62 & 90 & 421 & 992 & 122 & 87.6 & \\
\hline $17: 22$ & $2: 43$ & 62 & 90 & 411 & 991 & 113 & 81.1 & \\
\hline $17: 24$ & $2: 45$ & 62 & 89 & 410 & 996 & 115 & 82.6 & \\
\hline $17: 26$ & $2: 47$ & 53 & 88 & 419 & 992 & 111 & 79.7 & \\
\hline $17: 28$ & $2: 49$ & 54 & 88 & 429 & 992 & 134 & 96.2 & \\
\hline $17: 30$ & $2: 51$ & 59 & 90 & 410 & 992 & 124 & 89.0 & \\
\hline $17: 32$ & $2: 53$ & 53 & 91 & 409 & 991 & 111 & 79.7 & \\
\hline $17: 34$ & $2: 55$ & 53 & 90 & 431 & 990 & 112 & 80.4 & \\
\hline $17: 36$ & $2: 57$ & 59 & 88 & 416 & 990 & 117 & 84.0 & \\
\hline $17: 38$ & $2: 59$ & 53 & 89 & 413 & 992 & 120 & 86.1 & \\
\hline $17: 40$ & $3: 01$ & 59 & 90 & 430 & 993 & 132 & 94.8 & \\
\hline $17: 42$ & $3: 03$ & 54 & 90 & 412 & 990 & 115 & 82.6 & \\
\hline $17: 44$ & $3: 05$ & 57 & 89 & 430 & 993 & 120 & 86.1 & \\
\hline $17: 46$ & $3: 07$ & 53 & 89 & 412 & 997 & 113 & 81.1 & \\
\hline $17: 48$ & $3: 09$ & 54 & 89 & 415 & 993 & 132 & 94.8 & End Steady-state \\
\hline
\end{tabular}


MATR_11.XLS

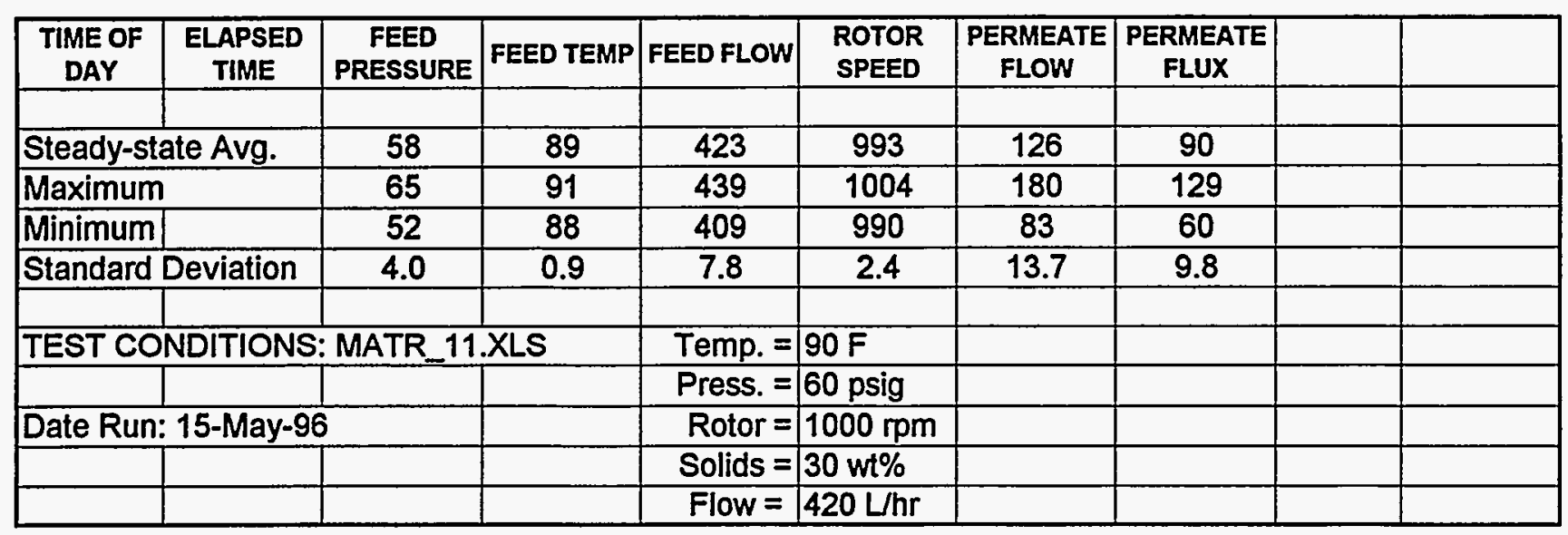


SpinTek II High Shear Rotary Membrane System

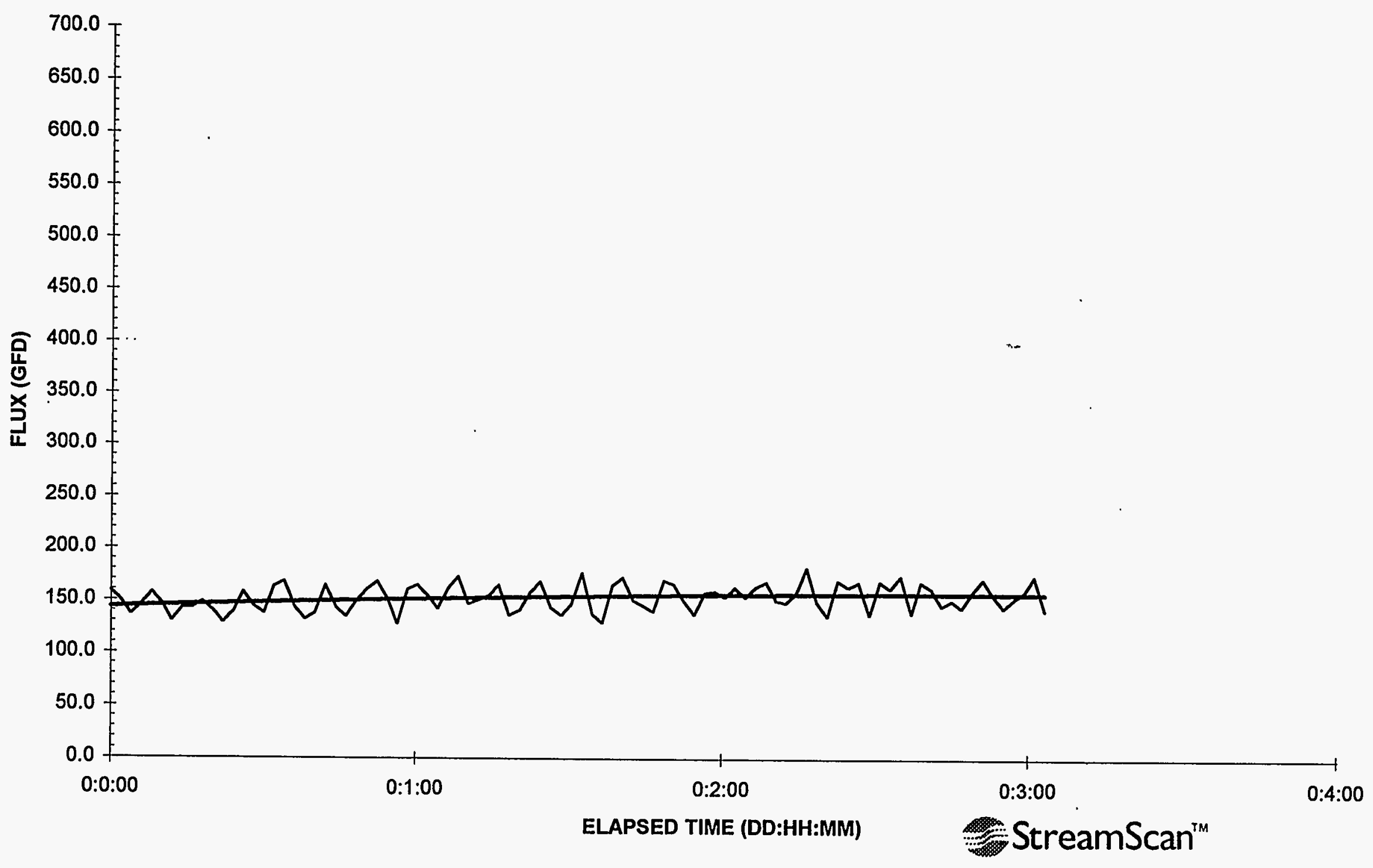




\begin{tabular}{|c|c|c|c|c|c|c|c|c|}
\hline $\begin{array}{c}\text { TIME OF } \\
\text { DAY }\end{array}$ & $\begin{array}{l}\text { ELAPSED } \\
\text { TIME }\end{array}$ & $\begin{array}{c}\text { FEED } \\
\text { PRESSURE }\end{array}$ & FEED TEMP & FEED FLOW & $\begin{array}{l}\text { ROTOR } \\
\text { SPEED }\end{array}$ & $\begin{array}{l}\text { PERMEATE } \\
\text { FLOW }\end{array}$ & $\begin{array}{c}\text { PERMEATE } \\
\text { FLUX } \\
\end{array}$ & \\
\hline $18: 33$ & $0: 00$ & 70 & 89 & 372 & 1494 & 221 & 158.6 & \\
\hline $18: 35$ & $0: 02$ & 70 & 91 & 368 & 1488 & 208 & 149.3 & \\
\hline $18: 37$ & $0: 04$ & 76 & 90 & 372 & 1489 & 190 & 136.4 & \\
\hline $18: 39$ & $0: 06$ & 70 & 88 & 372 & 1488 & 203 & 145.7 & \\
\hline $18: 41$ & $0: 08$ & 79 & 89 & 373 & 1489 & 219 & 157.2 & \\
\hline $18: 43$ & $0: 10$ & 71 & 90 & 371 & 1489 & 205 & 147.2 & \\
\hline $18: 45$ & $0: 12$ & 80 & 91 & 372 & 1489 & 181 & 129.9 & \\
\hline $18: 47$ & $0: 14$ & 71 & 89 & 368 & 1489 & 198 & 142.1 & \\
\hline $18: 49$ & $0: 16$ & 79 & 89 & 373 & 1489 & 199 & 142.8 & \\
\hline $18: 51$ & $0: 18$ & 70 & 90 & 369 & 1491 & 207 & 148.6 & \\
\hline $18: 53$ & $0: 20$ & 81 & 91 & 371 & 1489 & 196 & 140.7 & \\
\hline $18: 55$ & $0: 22$ & 69 & 89 & 369 & 1490 & 179 & 128.5 & \\
\hline $18: 57$ & $0: 24$ & 85 & 88 & 373 & 1497 & 194 & 139.3 & \\
\hline $18: 59$ & $0: 26$ & 72 & 89 & 368 & 1487 & 220 & 157.9 & \\
\hline $19: 02$ & $0: 28$ & 72 & 91 & 367 & 1489 & 201 & 144.3 & \\
\hline $19: 04$ & $0: 30$ & 77 & 89 & 364 & 1489 & 192 & 137.8 & \\
\hline $19: 06$ & $0: 32$ & 70 & 88 & 369 & 1488 & 228 & 163.7 & \\
\hline $19: 08$ & $0: 34$ & 78 & 89 & 368 & 1491 & 234 & 168.0 & \\
\hline $19: 10$ & $0: 36$ & 82 & 91 & 364 & 1488 & 200 & 143.6 & \\
\hline $19: 12$ & $0: 38$ & 73 & 90 & 366 & 1489 & 184 & 132.1 & \\
\hline $19: 14$ & $0: 40$ & 74 & 89 & 366 & 1490 & 192 & 137.8 & \\
\hline $19: 16$ & $0: 42$ & 70 & 89 & 368 & 1489 & 229 & 164.4 & \\
\hline $19: 18$ & $0: 44$ & 74 & 90 & 367 & 1489 & 200 & 143.6 & \\
\hline $19: 20$ & $0: 46$ & 83 & 91 & 365 & 1489 & 188 & 135.0 & \\
\hline $19: 22$ & $0: 48$ & 81 & 89 & 368 & 1490 & 208 & 149.3 & \\
\hline $19: 24$ & $0: 50$ & 71 & 88 & 366 & 1487 & 223 & 160.1 & \\
\hline $19: 26$ & $0: 52$ & 80 & 89 & 369 & 1494 & 234 & 168.0 & \\
\hline $19: 28$ & $0: 54$ & 73 & 91 & 365 & 1486 & 213 & 152.9 & \\
\hline $19: 30$ & $0: 56$ & 73 & 89 & 364 & 1488 & 178 & 127.8 & \\
\hline $19: 32$ & $0: 58$ & 71 & 88 & 367 & 1489 & 224 & 160.8 & \\
\hline $19: 34$ & $1: 00$ & 84 & 90 & 368 & 1488 & 230 & 165.1 & \\
\hline $19: 36$ & $1: 02$ & 72 & 90 & 363 & 1491 & 216 & 155.1 & Begin Steady-state \\
\hline $19: 38$ & $1: 04$ & 72 & 89 & 366 & 1492 & 198 & 142.1 & \\
\hline $19: 40$ & $1: 06$ & 74 & 89 & 367 & 1489 & 226 & 162.2 & \\
\hline $19: 42$ & $1: 08$ & 71 & 90 & 365 & 1489 & 241 & 173.0 & \\
\hline $19: 44$ & $1: 10$ & 72 & 91 & 364 & 1490 & 205 & 147.2 & \\
\hline $19: 46$ & $1: 12$ & 71 & 90 & 368 & 1492 & 210 & 150.7 & \\
\hline $19: 48$ & $1: 14$ & 78 & 89 & 368 & 1490 & 216 & 155.1 & \\
\hline $19: 50$ & $1: 16$ & 77 & 90 & 367 & 1488 & 230 & 165.1 & \\
\hline $19: 52$ & $1: 18$ & 80 & 91 & 365 & 1490 & 190 & 136.4 & \\
\hline $19: 54$ & $1: 20$ & 81 & 89 & 366 & 1488 & 197 & 141.4 & \\
\hline $19: 56$ & $1: 22$ & 73 & 89 & 365 & 1489 & 220 & 157.9 & \\
\hline 19:58 & $1: 24$ & 82 & 90 & 369 & 1488 & 234 & 168.0 & \\
\hline $20: 00$ & $1: 26$ & 82 & 91 & 364 & 1490 & 200 & 143.6 & \\
\hline $20: 02$ & $1: 28$ & 78 & 89 & 365 & 1490 & 190 & 136.4 & \\
\hline
\end{tabular}


MATR_12.XLS

\begin{tabular}{|c|c|c|c|c|c|c|c|c|}
\hline $\begin{array}{c}\text { TIME OF } \\
\text { DAY }\end{array}$ & $\begin{array}{c}\text { ELAPSED } \\
\text { TIME }\end{array}$ & \begin{tabular}{c|} 
FEED \\
PRESSURE \\
\end{tabular} & FEED TEMP & FEED FLOW & $\begin{array}{l}\text { ROTOR } \\
\text { SPEED }\end{array}$ & $\begin{array}{c}\text { PERMEATE } \\
\text { FLOW }\end{array}$ & $\begin{array}{l}\text { PERMEATE } \\
\text { FLUX }\end{array}$ & \\
\hline $20: 04$ & $1: 30$ & 79 & 89 & 364 & 1489 & 204 & 146.4 & \\
\hline $20: 06$ & $1: 32$ & 73 & 89 & 364 & 1490 & 246 & 176.6 & \\
\hline $20: 08$ & $1: 34$ & 81 & 91 & 362 & 1487 & 192 & 137.8 & \\
\hline $20: 10$ & $1: 36$ & 82 & 90 & 363 & 1489 & 180 & 129.2 & \\
\hline $20: 12$ & $1: 38$ & 81 & 88 & 363 & 1486 & 230 & 165.1 & \\
\hline $20: 14$ & $1: 40$ & 77 & 89 & 365 & 1489 & 240 & 172.3 & \\
\hline $20: 16$ & $1: 42$ & 85 & 90 & 365 & 1488 & 210 & 150.7 & \\
\hline $20: 18$ & $1: 44$ & 82 & 91 & 363 & $\overline{1496}$ & 203 & 145.7 & \\
\hline $20: 20$ & $1: 46$ & 72 & 89 & 364 & 1488 & 195 & 140.0 & \\
\hline $20: 22$ & $1: 48$ & 78 & 88 & 364 & 1493 & 236 & 169.4 & \\
\hline $20: 24$ & $1: 50$ & 80 & 89 & 363 & 1488 & 231 & 165.8 & \\
\hline $20: 26$ & $1: 52$ & 79 & 91 & 362 & 1489 & 209 & 150.0 & \\
\hline $20: 28$ & $1: 54$ & 85 & 90 & 360 & 1490 & 191 & 137.1 & \\
\hline $20: 30$ & $1: 56$ & 76 & 89 & 362 & 1489 & 220 & 157.9 & \\
\hline $20: 32$ & $1: 58$ & 75 & 89 & 364 & 1487 & 222 & 159.4 & \\
\hline $20: 34$ & $2: 00$ & 83 & 90 & 362 & 1490 & 214 & 153.6 & \\
\hline $20: 36$ & $2: 02$ & 79 & 90 & 360 & 1490 & 227 & 162.9 & \\
\hline $20: 38$ & $2: 04$ & 80 & 89 & 362 & 1488 & 214 & 153.6 & \\
\hline $20: 40$ & $2: 06$ & 76 & 89 & 361 & 1492 & 228 & 163.7 & \\
\hline $20: 42$ & $2: 08$ & 75 & 90 & 363 & 1489 & 234 & 168.0 & \\
\hline $20: 44$ & $2: 10$ & 81 & 91 & 361 & 1493 & 210 & 150.7 & \\
\hline $20: 46$ & $2: 12$ & 81 & 89 & 361 & 1492 & 207 & 148.6 & \\
\hline $20: 48$ & $2: 14$ & 75 & 88 & 363 & 1487 & 220 & 157.9 & \\
\hline $20: 50$ & $2: 16$ & 73 & 89 & 363 & 1491 & 253 & 181.6 & \\
\hline $20: 52$ & $2: 18$ & 73 & 90 & 362 & 1496 & 208 & 149.3 & \\
\hline $20: 54$ & $2: 20$ & 75 & 90 & 360 & 1491 & 189 & 135.7 & \\
\hline $20: 56$ & $2: 22$ & 72 & 88 & 363 & 1490 & 236 & 169.4 & \\
\hline $20: 58$ & $2: 24$ & 75 & 89 & 363 & 1492 & 228 & 163.7 & \\
\hline $21: 00$ & $2: 26$ & 80 & 90 & 359 & 1492 & 234 & 168.0 & \\
\hline $21: 02$ & $2: 29$ & 73 & 90 & 360 & 1491 & 191 & 137.1 & \\
\hline $21: 04$ & $2: 31$ & 76 & 88 & 363 & 1489 & 235 & 168.7 & \\
\hline $21: 06$ & $2: 33$ & 75 & 89 & 362 & 1487 & 226 & 162.2 & \\
\hline $21: 08$ & $2: 35$ & 75 & 90 & 361 & 1489 & 242 & 173.7 & \\
\hline $21: 10$ & $2: 37$ & 80 & 90 & 360 & 1489 & 193 & 138.5 & \\
\hline $21: 12$ & $2: 39$ & 80 & 88 & 364 & 1490 & 234 & 168.0 & \\
\hline $21: 14$ & $2: 41$ & 80 & 89 & 360 & 1490 & 226 & 162.2 & \\
\hline $21: 16$ & $2: 43$ & 80 & 90 & 360 & 1489 & 203 & 145.7 & \\
\hline $21: 18$ & $2: 45$ & 84 & 91 & 361 & 1489 & 210 & 150.7 & \\
\hline $21: 20$ & $2: 47$ & 84 & 90 & 362 & 1488 & 200 & 143.6 & \\
\hline $21: 23$ & $2: 49$ & 73 & 88 & 364 & 1493 & 221 & 158.6 & \\
\hline $21: 25$ & $2: 51$ & 75 & 89 & 361 & 1489 & 238 & 170.8 & \\
\hline $21: 27$ & $2: 53$ & 75 & 90 & 363 & 1489 & 217 & 155.8 & \\
\hline $21: 29$ & $2: 55$ & 82 & 90 & 362 & 1489 & 200 & 143.6 & \\
\hline $21: 31$ & $2: 57$ & 78 & 89 & 364 & 1487 & 213 & 152.9 & \\
\hline $21: 33$ & $2: 59$ & 72 & 88 & 364 & 1492 & 222 & 159.4 & \\
\hline $21: 35$ & $3: 01$ & 75 & 90 & 364 & 1489 & 242 & 173.7 & \\
\hline $21: 37$ & $3: 03$ & 78 & 91 & 360 & 1490 & 197 & 141.4 & End Steady-state \\
\hline
\end{tabular}


MATR_12.XLS

\begin{tabular}{|c|c|c|c|c|c|c|c|c|c|}
\hline $\begin{array}{c}\text { TIME OF } \\
\text { DAY }\end{array}$ & $\begin{array}{c}\text { ELAPSED } \\
\text { TIME } \\
\end{array}$ & $\begin{array}{c}\text { FEED } \\
\text { PRESSURE } \\
\end{array}$ & FEED TEMP & FEED FLOW & $\begin{array}{l}\text { ROTOR } \\
\text { SPEED }\end{array}$ & $\begin{array}{c}\text { PERMEATE } \\
\text { FLOW }\end{array}$ & \begin{tabular}{|c|} 
PERMEATE \\
FLUX
\end{tabular} & & \\
\hline \multicolumn{2}{|c|}{ Steady-state Avg. } & 77 & 90 & 363 & 1490 & 216 & 155 & & \\
\hline \multicolumn{2}{|c|}{ Maximum } & 85 & 91 & 369 & 1496 & 253 & 182 & & \\
\hline \multicolumn{2}{|c|}{ Minimum } & 71 & 88 & 359 & 1486 & 180 & 129 & & \\
\hline \multicolumn{2}{|c|}{ Standard Deviation } & 3.9 & 0.9 & 2.2 & 2.0 & 17.4 & 12.5 & & \\
\hline \multicolumn{4}{|c|}{ TEST CONDITIONS: MATR_12.XLS } & Temp. $=$ & $90 \mathrm{~F}$ & & & & \\
\hline & & & & Press. $=$ & 75 psig & & & & \\
\hline \multicolumn{3}{|c|}{ Date Run: 15-May-96 } & & Rotor $=$ & $1500 \mathrm{rpm}$ & & & & \\
\hline & & & & Solids $=$ & $30 w t \%$ & & & & \\
\hline & & & & Flow $=$ & $400 \mathrm{~L} / \mathrm{hr}$ & & & & \\
\hline
\end{tabular}

Page 3 


\section{SpinTek II High Shear Rotary Membrane System}

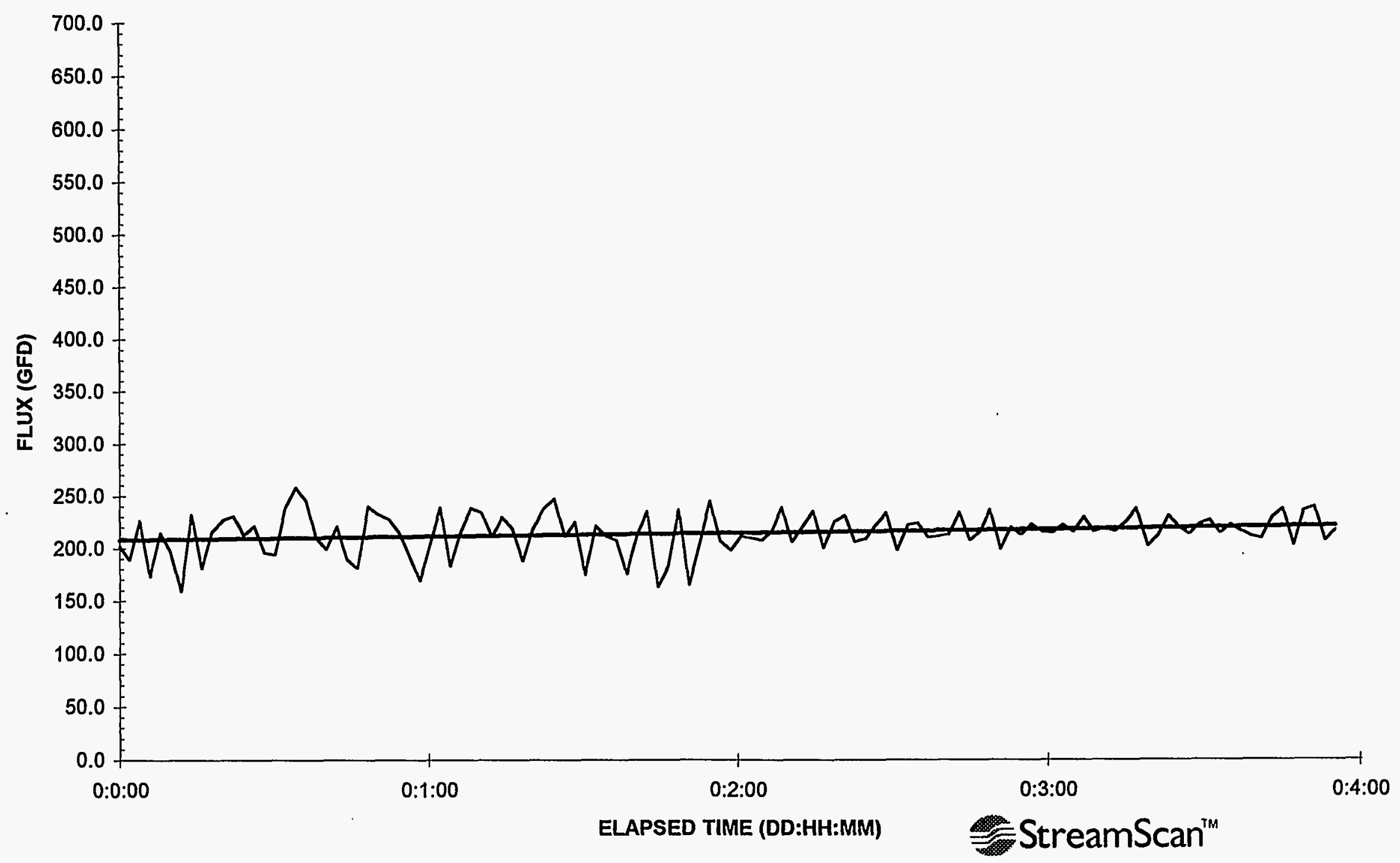




\begin{tabular}{|c|c|c|c|c|c|c|c|c|c|}
\hline $\begin{array}{l}\text { TIME OF } \\
\text { DAY }\end{array}$ & $\begin{array}{l}\text { ELAPSED } \\
\text { TIME }\end{array}$ & $\begin{array}{c}\text { FEED } \\
\text { PRESSURE }\end{array}$ & FEED TEMP & FEED FLOW & $\begin{array}{c}\text { ROTOR } \\
\text { SPEED }\end{array}$ & \begin{tabular}{|c|}
$\begin{array}{c}\text { PERMEATE } \\
\text { FLOW }\end{array}$ \\
\end{tabular} & \begin{tabular}{|c|}
$\begin{array}{c}\text { PERMEATE } \\
\text { FLUX }\end{array}$ \\
\end{tabular} & & \\
\hline $23: 15$ & $0: 00$ & 61 & 89 & 398 & 1507 & 281 & 201.7 & & \\
\hline $23: 17$ & $0: 02$ & 63 & 90 & 419 & 1503 & 263 & 188.8 & & \\
\hline $23: 19$ & $0: 04$ & 59 & 90 & 417 & 1509 & 315 & 226.1 & & \\
\hline $23: 21$ & $0: 06$ & 64 & 91 & 404 & 1510 & 241 & 173.0 & & \\
\hline $23: 23$ & $0: 08$ & 66 & 90 & 417 & 1499 & 299 & 214.6 & & \\
\hline $23: 25$ & $0: 10$ & 63 & 89 & 421 & 1508 & 274 & 196.7 & & \\
\hline 23:27 & $0: 12$ & 58 & 91 & 419 & 1503 & 221 & 158.6 & & \\
\hline $23: 29$ & $0: 14$ & 65 & 90 & 399 & 1497 & 323 & 231.9 & & \\
\hline $23: 31$ & $0: 16$ & 57 & 89 & 426 & 1503 & 252 & 180.9 & & \\
\hline $23: 33$ & $0: 18$ & 62 & 91 & 424 & 1505 & 301 & 216.1 & & \\
\hline $23: 35$ & $0: 20$ & 63 & 90 & 443 & 1507 & 316 & 226.8 & & \\
\hline $23: 37$ & $0: 22$ & 61 & 90 & 414 & 1502 & 321 & 230.4 & & \\
\hline $23: 39$ & $0: 24$ & 64 & 90 & 427 & 1498 & 296 & 212.5 & & \\
\hline $23: 41$ & $0: 26$ & 64 & 89 & 411 & 1506 & 308 & 221.1 & & \\
\hline $23: 43$ & $0: 28$ & 58 & 89 & 411 & 1501 & 273 & 196.0 & & \\
\hline $23: 45$ & $0: 30$ & 63 & 91 & 421 & 1507 & 270 & 193.8 & & \\
\hline $23: 47$ & $0: 32$ & 57 & 90 & 422 & 1497 & 332 & 238.3 & & \\
\hline $23: 49$ & $0: 34$ & 62 & 89 & 406 & 1507 & 359 & 257.7 & & \\
\hline $23: 51$ & $0: 36$ & 63 & 91 & 407 & 1497 & 342 & 245.5 & & \\
\hline $23: 53$ & $0: 38$ & 58 & 90 & 427 & 1509 & 293 & 210.3 & & \\
\hline $23: 55$ & $0: \overline{40}$ & 61 & 89 & 421 & 1509 & 277 & 198.8 & & \\
\hline $23: 58$ & $0: 42$ & 60 & 90 & 418 & 1509 & 307 & 220.4 & & \\
\hline $0: 00$ & $0: 44$ & 58 & 90 & 407 & 1499 & 264 & 189.5 & & \\
\hline $0: 02$ & $0: 46$ & 57 & 89 & 436 & 1501 & 252 & 180.9 & & \\
\hline $0: 04$ & $0: 48$ & 63 & 90 & 409 & 1503 & 334 & 239.8 & & \\
\hline $0: 06$ & $0: 50$ & 62 & 91 & 409 & 1498 & 324 & 232.6 & & \\
\hline $0: 08$ & $0: 52$ & 64 & 90 & 420 & 1507 & 317 & 227.6 & & \\
\hline $0: 10$ & $0: 54$ & 60 & 89 & 408 & 1518 & 298 & 213.9 & & \\
\hline $0: 12$ & $0: 56$ & 57 & 91 & 411 & 1502 & 268 & 192.4 & & \\
\hline $0: 14$ & $0: 58$ & 57 & 91 & 399 & 1507 & 236 & 169.4 & & \\
\hline $0: 16$ & $1: 00$ & 63 & 89 & 411 & 1503 & 284 & 203.9 & & \\
\hline $0: 18$ & $1: 02$ & 59 & 90 & 423 & 1499 & 332 & 238.3 & & \\
\hline $0: 20$ & $1: 04$ & 66 & 91 & 405 & 1500 & 255 & 183.0 & & \\
\hline $0: 22$ & $1: 06$ & 59 & 89 & 404 & 1503 & 301 & 216.1 & & \\
\hline $0: 24$ & $1: 08$ & 66 & 89 & 417 & 1493 & 332 & 238.3 & & \\
\hline $0: 26$ & $1: 10$ & 64 & 90 & 412 & 1502 & 326 & 234.0 & & \\
\hline $0: 28$ & $1: 12$ & 62 & 90 & 411 & 1502 & 294 & 211.0 & & \\
\hline $0: 30$ & $1: 14$ & 61 & 89 & 426 & 1496 & 320 & 229.7 & & \\
\hline $0: 32$ & $1: 16$ & 65 & 90 & 419 & 1502 & 305 & 218.9 & & \\
\hline $0: 34$ & $1: 18$ & 58 & 91 & 401 & 1499 & 262 & 188.1 & & \\
\hline $0: 36$ & $1: 20$ & 62 & 89 & 414 & 1509 & 306 & 219.7 & & \\
\hline $0: 38$ & $1: 22$ & 61 & 90 & 437 & 1502 & 331 & 237.6 & & \\
\hline $0: 40$ & $1: 24$ & 66 & 90 & 409 & 1510 & 344 & 246.9 & & \\
\hline $0: 42$ & $1: 26$ & 61 & 89 & 407 & 1503 & 294 & 211.0 & & \\
\hline $0: 44$ & $1: 28$ & 61 & 90 & 407 & 1503 & 313 & 224.7 & & \\
\hline
\end{tabular}


MATR_13.XLS

\begin{tabular}{|c|c|c|c|c|c|c|c|c|}
\hline $\begin{array}{c}\text { TIME OF } \\
\text { DAY }\end{array}$ & $\begin{array}{l}\text { ELAPSED } \\
\text { TIME }\end{array}$ & $\begin{array}{c}\text { FEED } \\
\text { PRESSURE } \\
\end{array}$ & FEED TEMP & FEED FLOW & $\begin{array}{l}\text { ROTOR } \\
\text { SPEED } \\
\end{array}$ & $\begin{array}{c}\text { PERMEATE } \\
\text { FLOW }\end{array}$ & \begin{tabular}{|c|} 
PERMEATE \\
FLUX
\end{tabular} & \\
\hline $0: 46$ & $1: 30$ & 63 & 91 & 412 & 1508 & 243 & 174.4 & \\
\hline $0: 48$ & $1: 32$ & 62 & 89 & 414 & 1507 & 308 & 221.1 & \\
\hline $0: 50$ & $1: 34$ & 59 & 89 & 426 & 1506 & 294 & 211.0 & \\
\hline $0: 52$ & $1: 36$ & 66 & 90 & 418 & 1502 & 289 & 207.5 & \\
\hline $0: 54$ & $1: 38$ & 63 & 89 & 402 & 1504 & 244 & 175.2 & \\
\hline $0: 56$ & $1: 40$ & 61 & 90 & 426 & 1504 & 297 & 213.2 & \\
\hline $0: 58$ & $1: 42$ & 59 & 91 & 412 & 1501 & 327 & 234.7 & \\
\hline $1: 00$ & $1: 44$ & 59 & 90 & 429 & 1506 & 227 & 162.9 & \\
\hline $1: 02$ & $1: 46$ & 62 & 90 & 407 & 1502 & 254 & 182.3 & \\
\hline $1: 04$ & $1: 48$ & 59 & 90 & 406 & 1506 & 329 & 236.2 & \\
\hline $1: 06$ & $1: 50$ & 57 & 91 & 403 & 1496 & 230 & 165.1 & \\
\hline $1: 08$ & $1: 52$ & 57 & 89 & 413 & 1508 & 284 & 203.9 & \\
\hline $1: 10$ & $1: 54$ & 58 & 89 & 420 & 1498 & 341 & 244.8 & \\
\hline $1: 12$ & $1: 56$ & 67 & 90 & 415 & 1500 & 288 & 206.7 & \\
\hline $1: 14$ & $1: 58$ & 58 & 89 & 406 & 1500 & 276 & 198.1 & \\
\hline $1: 16$ & $2: 00$ & 63 & 90 & 417 & 1509 & 294 & 211.0 & Begin Steady-state \\
\hline $1: 18$ & $2: 02$ & 58 & 91 & 409 & 1501 & 292 & 209.6 & \\
\hline $1: 20$ & $2: 04$ & 57 & 90 & 419 & 1510 & 289 & 207.5 & \\
\hline $1: 22$ & $2: 06$ & 59 & 90 & 411 & 1503 & 302 & 216.8 & \\
\hline $1: 24$ & $2: 08$ & 60 & 90 & 397 & 1501 & 332 & 238.3 & \\
\hline $1: 26$ & $2: 10$ & 60 & 91 & 408 & 1503 & 287 & 206.0 & \\
\hline $1: 28$ & $2: 12$ & 68 & 89 & 420 & 1506 & 308 & 221.1 & \\
\hline $1: 30$ & $2: 14$ & 62 & 90 & 419 & 1504 & 328 & 235.4 & \\
\hline $1: 32$ & $2: 16$ & 61 & 90 & 411 & 1510 & 279 & 200.3 & \\
\hline $1: 34$ & $2: 18$ & 58 & 89 & 418 & 1501 & 313 & 224.7 & \\
\hline $1: 36$ & $2: 20$ & 63 & 90 & 406 & 1513 & 322 & 231.1 & \\
\hline $1: 38$ & $2: 22$ & 67 & 91 & 400 & 1501 & 287 & 206.0 & \\
\hline $1: 40$ & $2: 24$ & 65 & 89 & 415 & 1503 & 291 & 208.9 & \\
\hline $1: 42$ & $2: 26$ & 58 & 89 & 416 & 1500 & 309 & 221.8 & \\
\hline $1: 44$ & $2: 28$ & 67 & 90 & 409 & 1508 & 325 & 233.3 & \\
\hline $1: 46$ & $2: 30$ & 66 & 90 & 412 & 1502 & 276 & 198.1 & \\
\hline $1: 48$ & $2: 32$ & 60 & 89 & 416 & 1506 & 309 & 221.8 & \\
\hline $1: 50$ & $2: 34$ & 60 & 90 & 416 & 1499 & 312 & 224.0 & \\
\hline $1: 52$ & $2: 36$ & 58 & 91 & 413 & 1497 & 293 & 210.3 & \\
\hline $1: 54$ & $2: 39$ & 58 & 90 & 402 & 1506 & 294 & 211.0 & \\
\hline $1: 56$ & $2: 41$ & 58 & 89 & 418 & 1501 & 297 & 213.2 & \\
\hline $1: 58$ & $2: 43$ & 62 & 90 & 417 & 1500 & 326 & 234.0 & \\
\hline $2: 00$ & $2: 45$ & 65 & 91 & 411 & 1503 & 289 & 207.5 & \\
\hline $2: 02$ & $2: 47$ & 59 & 89 & 409 & 1508 & 300 & 215.3 & \\
\hline $2: 04$ & $2: 49$ & 60 & 90 & 412 & 1493 & 329 & 236.2 & \\
\hline $2: 06$ & $2: 51$ & 64 & 90 & 424 & 1505 & 277 & 198.8 & \\
\hline $2: 08$ & $2: 53$ & 59 & 89 & 410 & 1507 & 306 & 219.7 & \\
\hline $2: 10$ & $2: 55$ & 61 & 89 & 410 & 1509 & 296 & 212.5 & \\
\hline $2: 12$ & $2: 57$ & 58 & 91 & 407 & 1503 & 310 & 222.5 & \\
\hline $2: 14$ & $2: 59$ & 67 & 90 & 404 & 1497 & 300 & 215.3 & \\
\hline $2: 16$ & $3: 01$ & 56 & 89 & 404 & 1493 & 299 & 214.6 & \\
\hline $2: 18$ & $3: 03$ & 58 & 90 & 421 & 1510 & 309 & 221.8 & \\
\hline $2: 20$ & $3: 05$ & 67 & 91 & 413 & 1505 & 300 & 215.3 & \\
\hline $2: 22$ & $3: 07$ & 68 & 89 & 403 & 1502 & 320 & 229.7 & \\
\hline $2: 24$ & $3: 09$ & 60 & 89 & 417 & 1500 & 300 & 215.3 & \\
\hline
\end{tabular}


MATR_13.XLS

\begin{tabular}{|c|c|c|c|c|c|c|c|c|}
\hline $\begin{array}{c}\text { TIME OF } \\
\text { DAY }\end{array}$ & $\begin{array}{c}\text { ELAPSED } \\
\text { TIME } \\
\end{array}$ & \begin{tabular}{|c|} 
FEED \\
PRESSURE \\
\end{tabular} & FEED TEMP & FEED FLOW & $\begin{array}{l}\text { ROTOR } \\
\text { SPEED } \\
\end{array}$ & $\begin{array}{l}\text { PERMEATE } \\
\text { FLOW }\end{array}$ & $\begin{array}{c}\text { PERMEATE } \\
\text { FLUX }\end{array}$ & \\
\hline $2: 26$ & $3: 11$ & 56 & 90 & 409 & 1507 & 305 & 218.9 & \\
\hline $2: 28$ & $3: 13$ & 63 & 90 & 399 & 1495 & 301 & 216.1 & \\
\hline $2: 30$ & $3: 15$ & 60 & 89 & 407 & 1506 & 313 & 224.7 & \\
\hline $2: 32$ & $3: 17$ & 60 & 90 & 412 & 1502 & 331 & 237.6 & \\
\hline $2: 34$ & $3: 19$ & 63 & 91 & 404 & 1506 & 281 & 201.7 & \\
\hline $2: 36$ & $3: 21$ & 66 & 88 & 405 & 1498 & 295 & 211.8 & \\
\hline $2: 38$ & $3: 23$ & 67 & 89 & 412 & 1505 & 321 & 230.4 & \\
\hline $2: 40$ & $3: 25$ & 60 & 90 & 425 & 1503 & 306 & 219.7 & \\
\hline $2: 42$ & $3: 27$ & 63 & 90 & 392 & 1504 & 297 & 213.2 & \\
\hline $2: 44$ & $3: 29$ & 56 & 89 & 420 & 1503 & 310 & 222.5 & \\
\hline $2: 46$ & $3: 31$ & 59 & 90 & 418 & 1506 & 315 & 226.1 & \\
\hline $2: 48$ & $3: 33$ & 59 & 90 & 400 & 1502 & 298 & 213.9 & \\
\hline $2: 51$ & $3: 35$ & 60 & 89 & 418 & 1508 & 309 & 221.8 & \\
\hline $2: 53$ & $3: 37$ & 59 & 90 & 407 & 1508 & 301 & 216.1 & \\
\hline $2: 55$ & $3: 39$ & 59 & 90 & 408 & 1508 & 294 & 211.0 & \\
\hline $2: 57$ & $3: 41$ & 60 & 89 & 422 & 1508 & 291 & 208.9 & \\
\hline $2: 59$ & $3: 43$ & 58 & 89 & 418 & 1500 & 319 & 229.0 & \\
\hline $3: 01$ & $3: 45$ & 68 & 90 & 406 & 1503 & 330 & 236.9 & \\
\hline $3: 03$ & $3: 47$ & 59 & 90 & 406 & 1498 & 282 & 202.4 & \\
\hline $3: 05$ & $3: 49$ & 67 & 89 & 418 & 1494 & 328 & 235.4 & \\
\hline $3: 07$ & $3: 51$ & 64 & 90 & 419 & 1505 & 333 & 239.0 & \\
\hline $3: 09$ & $3: 53$ & 61 & 91 & 421 & 1502 & 288 & 206.7 & \\
\hline $3: 11$ & $3: 55$ & 56 & 88 & 406 & 1505 & 302 & 216.8 & End Steady-state \\
\hline \multicolumn{2}{|c|}{ Steady-state Avg. } & 61 & 90 & 411 & 1503 & 304 & 218 & \\
\hline \multicolumn{2}{|l|}{ Maximum } & 68 & 91 & 425 & 1513 & 333 & 239 & \\
\hline Minimum & & 56 & 88 & 392 & 1493 & 276 & 198 & \\
\hline \multicolumn{2}{|c|}{ Standard Deviation } & 3.6 & 0.8 & 7.2 & 4.4 & 15.2 & 10.9 & \\
\hline & & & & & & & & \\
\hline \multicolumn{4}{|c|}{ TEST CONDITIONS: MATR_13.XLS } & Temp. $=$ & $90^{\circ} \mathrm{F}$ & & & \\
\hline & & & & Press. $=$ & $60 \mathrm{psig}$ & & & \\
\hline \multicolumn{3}{|c|}{ Date Run: 16-May-96 } & & Rotor $=$ & $1500 \mathrm{rpm}$ & & & \\
\hline & & & & Solids $=$ & $15 w t \%$ & & & \\
\hline & & & & Flow $=$ & $400 \mathrm{~L} / \mathrm{hr}$ & & & \\
\hline
\end{tabular}

Page 3 


\section{SpinTek II High Shear Rotary Membrane System}

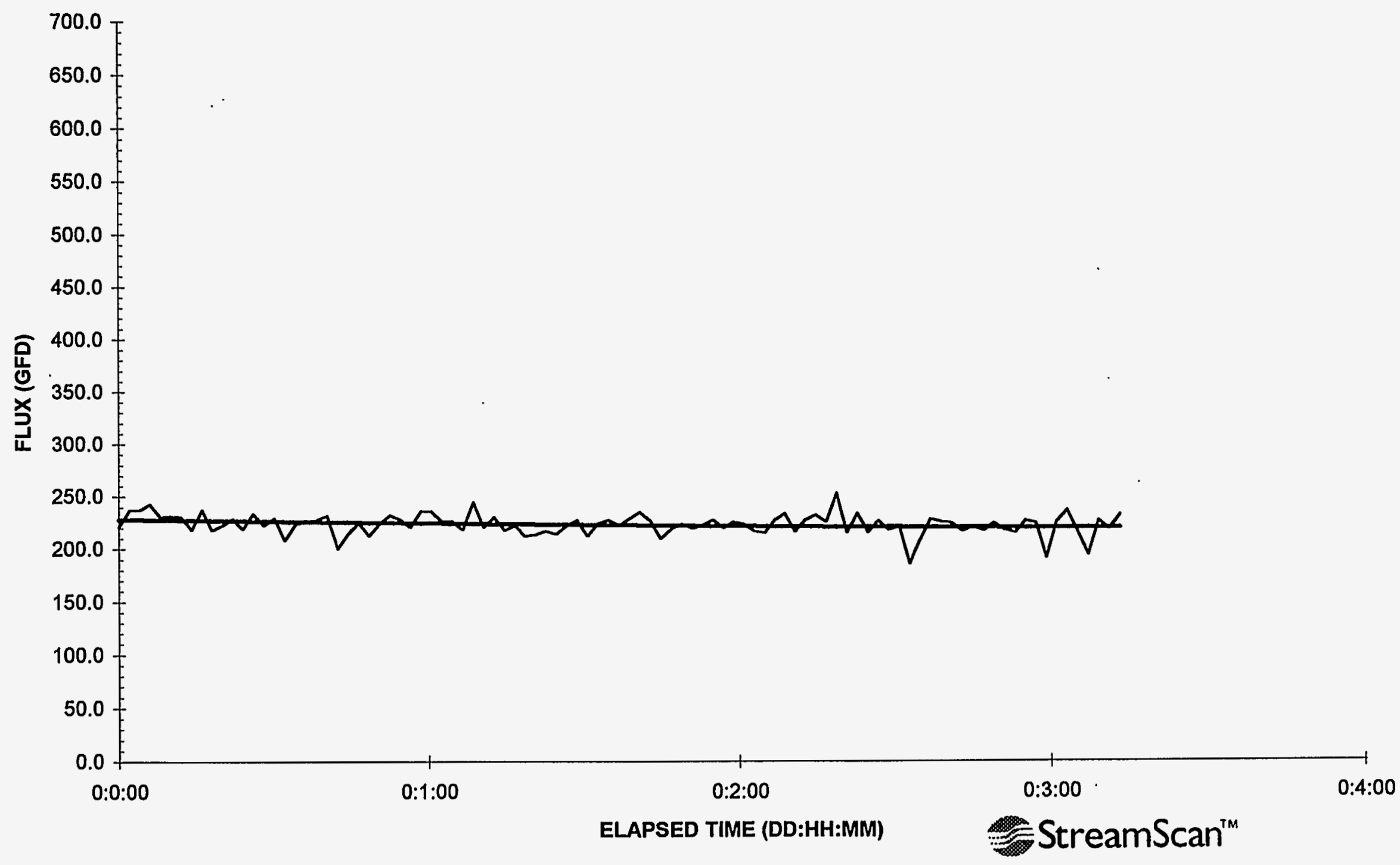




\begin{tabular}{|c|c|c|c|c|c|c|c|c|}
\hline $\begin{array}{c}\text { TIME OF } \\
\text { DAY }\end{array}$ & $\begin{array}{c}\text { ELAPSED } \\
\text { TIME }\end{array}$ & $\begin{array}{c}\text { FEED } \\
\text { PRESSURE }\end{array}$ & FEED TEMP & FEED FLOW & $\begin{array}{l}\text { ROTOR } \\
\text { SPEED }\end{array}$ & $\begin{array}{c}\text { PERMEATE } \\
\text { FLOW }\end{array}$ & \begin{tabular}{|c} 
PERMEATE \\
FLUX
\end{tabular} & \\
\hline $4: 55$ & $0: 00$ & 62 & 117 & 451 & 999 & 308 & 221.1 & \\
\hline $4: 57$ & $0: 02$ & 61 & 118 & 460 & 999 & 330 & 236.9 & \\
\hline $4: 59$ & $0: 04$ & 59 & 118 & 465 & 997 & 330 & 236.9 & \\
\hline $5: 01$ & $0: 06$ & 60 & 119 & 467 & 1000 & 338 & 242.6 & \\
\hline $5: 03$ & $0: 08$ & 60 & 119 & 456 & 999 & 321 & 230.4 & \\
\hline $5: 05$ & $0: 10$ & 67 & 119 & 470 & 1002 & 322 & 231.1 & \\
\hline 5:07 & $0: 12$ & 59 & 119 & 472 & 1004 & 321 & 230.4 & \\
\hline $5: 09$ & $0: 14$ & 59 & 120 & 475 & 997 & 304 & 218.2 & \\
\hline $5: 11$ & $0: 16$ & 59 & 120 & 465 & 998 & 330 & 236.9 & \\
\hline $5: 13$ & $0: 18$ & 61 & 119 & 465 & 997 & 303 & 217.5 & \\
\hline $5: 15$ & $0: 20$ & 61 & 117 & 479 & 999 & 310 & 222.5 & \\
\hline $5: 17$ & $0: 22$ & 65 & 117 & 470 & 997 & 318 & 228.3 & \\
\hline $5: 19$ & $0: 24$ & 64 & 117 & 453 & 999 & 305 & 218.9 & \\
\hline $5: 21$ & $0: 26$ & 62 & 118 & 462 & 1001 & 325 & 233.3 & \\
\hline $5: 23$ & $0: 28$ & 65 & 118 & 490 & 997 & 310 & 222.5 & \\
\hline $5: 25$ & $0: 30$ & 63 & 119 & 470 & 997 & 319 & 229.0 & \\
\hline $5: 27$ & $0: 32$ & 58 & 119 & 455 & 998 & 290 & 208.2 & \\
\hline $5: 29$ & $0: 34$ & 58 & 119 & 477 & 999 & 311 & 223.2 & \\
\hline $5: 32$ & $0: 36$ & 62 & 119 & 474 & 1003 & 315 & 226.1 & \\
\hline $5: 34$ & $0: 38$ & 58 & 120 & 466 & 1001 & 317 & 227.6 & \\
\hline $5: 36$ & $0: 40$ & 60 & 120 & 469 & 996 & 322 & 231.1 & \\
\hline $5: 38$ & $0: 42$ & 62 & 120 & 449 & 997 & 279 & 200.3 & \\
\hline $5: 40$ & $0: 44$ & 57 & 118 & 463 & 998 & 298 & 213.9 & \\
\hline $5: 42$ & $0: 46$ & 59 & 117 & 473 & 1000 & 313 & 224.7 & \\
\hline $5: 44$ & $0: 48$ & 63 & 118 & 465 & 1001 & 296 & 212.5 & \\
\hline $5: 46$ & $0: 50$ & 56 & 118 & 481 & 997 & 312 & 224.0 & \\
\hline $5: 48$ & $0: 52$ & 64 & 118 & 459 & 997 & 323 & 231.9 & \\
\hline $5: 50$ & $0: 54$ & 57 & 119 & 464 & 1000 & 317 & 227.6 & \\
\hline $5: 52$ & $0: 56$ & 57 & 119 & 477 & 998 & 307 & 220.4 & \\
\hline $5: 54$ & $0: 58$ & 61 & 119 & 473 & 997 & 328 & 235.4 & \\
\hline $5: 56$ & $1: 00$ & 61 & 119 & 480 & 997 & 328 & 235.4 & \\
\hline $5: 58$ & $1: 02$ & 58 & 120 & 473 & 998 & 315 & 226.1 & \\
\hline $6: 00$ & $1: 04$ & 58 & 120 & 485 & 997 & 315 & 226.1 & \\
\hline $6: 02$ & $1: 06$ & 63 & 120 & 480 & 1000 & 304 & 218.2 & \\
\hline $6: 04$ & $1: 08$ & 66 & 120 & 485 & 997 & 340 & 244.1 & \\
\hline $6: 06$ & $1: 10$ & 58 & 121 & 472 & 1000 & 307 & 220.4 & \\
\hline $6: 08$ & $1: 12$ & 58 & 121 & 459 & 999 & 320 & 229.7 & \\
\hline $6: 10$ & $1: 14$ & 61 & 119 & 470 & 997 & 303 & 217.5 & Begin Steady-state \\
\hline $6: 12$ & $1: 16$ & 60 & 118 & 470 & 998 & 309 & 221.8 & \\
\hline $6: 14$ & $1: 18$ & 59 & 118 & 473 & 1000 & 295 & 211.8 & \\
\hline $6: 16$ & $1: 20$ & 57 & 118 & 476 & 998 & 297 & 213.2 & \\
\hline $6: 18$ & $1: 22$ & 56 & 118 & 466 & 1000 & 302 & 216.8 & \\
\hline $6: 20$ & $1: 24$ & 58 & 119 & 480 & 997 & 298 & 213.9 & \\
\hline $6: 22$ & $1: 26$ & 57 & 119 & 478 & 998 & 309 & 221.8 & \\
\hline $6: 24$ & $1: 28$ & 58 & 119 & 470 & 997 & 316 & 226.8 & \\
\hline
\end{tabular}


MATR_14.XLS

\begin{tabular}{|c|c|c|c|c|c|c|c|c|c|}
\hline $\begin{array}{c}\text { TIME OF } \\
\text { DAY }\end{array}$ & $\begin{array}{c}\text { ELAPSED } \\
\text { TIME }\end{array}$ & $\begin{array}{c}\text { FEED } \\
\text { PRESSURE }\end{array}$ & FEED TEMP & FEED FLOW & $\begin{array}{l}\text { ROTOR } \\
\text { SPEED }\end{array}$ & $\begin{array}{l}\text { PERMEATE } \\
\text { FLOW }\end{array}$ & $\begin{array}{c}\text { PERMEATE } \\
\text { FLUX }\end{array}$ & & \\
\hline $6: 26$ & $1: 30$ & 58 & 119 & 468 & 998 & 295 & 211.8 & & \\
\hline $6: 28$ & $1: 32$ & 58 & 120 & 445 & 1001 & 312 & 224.0 & & \\
\hline $6: 30$ & $1: 34$ & 60 & 120 & 464 & 999 & 316 & 226.8 & & \\
\hline $6: 32$ & $1: 36$ & 61 & 120 & 489 & 999 & 309 & 221.8 & & \\
\hline $6: 34$ & $1: 38$ & 58 & 121 & 477 & 1000 & 318 & 228.3 & & \\
\hline $6: 36$ & $1: 40$ & 61 & 121 & 483 & 997 & 327 & 234.7 & & \\
\hline $6: 38$ & $1: 42$ & 57 & 121 & 456 & 997 & 315 & 226.1 & & \\
\hline $6: 40$ & $1: 44$ & 58 & 120 & 472 & 995 & 292 & 209.6 & & \\
\hline $6: 42$ & $1: 46$ & 58 & 118 & 471 & 997 & 305 & 218.9 & & \\
\hline $6: 44$ & $1: 48$ & 64 & 118 & 460 & 998 & 311 & 223.2 & & \\
\hline $6: 46$ & $1: 50$ & 56 & 119 & 470 & 999 & 305 & 218.9 & & \\
\hline $6: 48$ & $1: 52$ & 60 & 119 & 470 & 1000 & 310 & 222.5 & & \\
\hline $6: 50$ & $1: 54$ & 63 & 119 & 480 & 997 & 316 & 226.8 & & \\
\hline $6: 52$ & $1: 56$ & 63 & 119 & 471 & 1001 & 306 & 219.7 & & \\
\hline $6: 54$ & $1: 58$ & 59 & 120 & 455 & 997 & 314 & 225.4 & & \\
\hline $6: 56$ & $2: 00$ & 62 & 120 & 456 & 998 & 311 & 223.2 & & \\
\hline $6: 58$ & $2: 02$ & 63 & 120 & 492 & 997 & 302 & 216.8 & & \\
\hline $7: 00$ & $2: 05$ & 58 & 120 & 462 & 997 & 299 & 214.6 & & \\
\hline $7: 02$ & $2: 07$ & 62 & 121 & 463 & 998 & 316 & 226.8 & & \\
\hline $7: 04$ & $2: 09$ & 56 & 121 & 467 & 998 & 325 & 233.3 & & \\
\hline $7: 06$ & $2: 11$ & 62 & 121 & 473 & 1000 & 301 & 216.1 & & \\
\hline $7: 08$ & $2: 13$ & 56 & 119 & 467 & 999 & 316 & 226.8 & & \\
\hline $7: 10$ & $2: 15$ & 62 & 119 & 471 & 997 & 323 & 231.9 & & \\
\hline $7: 12$ & $2: 17$ & 56 & 119 & 478 & 998 & 314 & 225.4 & & \\
\hline $7: 14$ & $2: 19$ & 61 & 119 & 479 & 997 & 352 & 252.7 & & \\
\hline $7: 16$ & $2: 21$ & 59 & 120 & 451 & 998 & 300 & 215.3 & & \\
\hline $7: 18$ & $2: 23$ & 61 & 120 & 472 & 998 & 325 & 233.3 & & \\
\hline $7: 20$ & $2: 25$ & 57 & 120 & 472 & 997 & 300 & 215.3 & & \\
\hline $7: 22$ & $2: 27$ & 58 & 121 & 460 & 995 & 315 & 226.1 & & \\
\hline $7: 24$ & $2: 29$ & 56 & 120 & 473 & 996 & 303 & 217.5 & & \\
\hline $7: 26$ & $2: 31$ & 61 & 121 & 481 & 996 & 307 & 220.4 & & \\
\hline $7: 28$ & $2: 33$ & 56 & 120 & 470 & 997 & 258 & 185.2 & & \\
\hline $7: 30$ & $2: 35$ & 60 & 120 & 465 & 997 & 290 & 208.2 & & \\
\hline $7: 32$ & $2: 37$ & 56 & 120 & 465 & 999 & 317 & 227.6 & & \\
\hline $7: 34$ & $2: 39$ & 56 & 120 & 464 & 997 & 314 & 225.4 & & \\
\hline $7: 36$ & $2: 41$ & 61 & 120 & 479 & 997 & 312 & 224.0 & & \\
\hline $7: 38$ & $2: 43$ & 56 & 121 & 499 & 1002 & 301 & 216.1 & & \\
\hline $7: 40$ & $2: 45$ & 56 & 120 & 452 & 1000 & 307 & 220.4 & & \\
\hline $7: 42$ & $2: 47$ & 57 & 119 & 475 & 998 & 302 & 216.8 & & \\
\hline $7: 44$ & $2: 49$ & 57 & 119 & 471 & 999 & 312 & 224.0 & & \\
\hline $7: 46$ & $2: 51$ & 58 & 120 & 471 & 996 & 303 & 217.5 & & \\
\hline $7: 48$ & $2: 53$ & 64 & 120 & 458 & 997 & 299 & 214.6 & & \\
\hline $7: 50$ & $2: 55$ & 56 & 120 & 471 & 997 & 315 & 226.1 & & \\
\hline $7: 52$ & $2: 57$ & 64 & 120 & 463 & 997 & 312 & 224.0 & & \\
\hline $7: 54$ & $2: 59$ & 56 & 121 & 481 & 999 & 266 & 190.9 & & \\
\hline $7: 56$ & $3: 01$ & 65 & 121 & 467 & 999 & 314 & 225.4 & & \\
\hline $7: 58$ & $3: 03$ & 57 & 121 & 464 & 995 & 329 & 236.2 & & \\
\hline $8: 01$ & $3: 05$ & 61 & 121 & 463 & 997 & 299 & 214.6 & & \\
\hline $8: 03$ & $3: 07$ & 56 & 120 & 472 & 1000 & 270 & 193.8 & & \\
\hline $8: 05$ & $3: 09$ & 63 & 118 & 473 & 997 & 315 & 226.1 & & \\
\hline
\end{tabular}


MATR_14.XLS

\begin{tabular}{|c|c|c|c|c|c|c|c|c|}
\hline $\begin{array}{c}\text { TIME OF } \\
\text { DAY }\end{array}$ & $\begin{array}{l}\text { ELAPSED } \\
\text { TIME }\end{array}$ & $\begin{array}{c}\text { FEED } \\
\text { PRESSURE }\end{array}$ & FEED TEMP & FEED FLOW & $\begin{array}{l}\text { ROTOR } \\
\text { SPEED } \\
\end{array}$ & $\begin{array}{c}\text { PERMEATE } \\
\text { FLOW }\end{array}$ & \begin{tabular}{|c|} 
PERMEATE \\
FLUX
\end{tabular} & \\
\hline $8: 07$ & $3: 11$ & 56 & 118 & $\overline{478}$ & 999 & 304 & 218.2 & \\
\hline $8: 09$ & $3: 13$ & 56 & 119 & 487 & 998 & 323 & 231.9 & End Steady-state \\
\hline & & & & & & & & \\
\hline \multicolumn{2}{|c|}{ Steady-state Avg. } & 59 & 120 & 470 & 998 & 308 & 221 & \\
\hline \multicolumn{2}{|c|}{ Maximum } & 65 & 121 & 499 & 1002 & 352 & 253 & \\
\hline Minimum & & 56 & 118 & 445 & 995 & 258 & 185 & \\
\hline \multicolumn{2}{|c|}{ Standard Deviation } & 2.7 & 1.0 & 9.9 & 1.5 & 14.4 & 10.3 & \\
\hline & & & & & & & & \\
\hline \multicolumn{4}{|c|}{ TEST CONDITIONS: MATR_14.XLS } & Temp. $=$ & $120^{\circ} \mathrm{F}$ & & & \\
\hline & & & & Press. $=$ & 60 psig & & & \\
\hline \multicolumn{3}{|c|}{ Date Run: 16-May-96 } & & Rotor $=$ & $1000 \mathrm{rpm}$ & & & \\
\hline & & & & Solids $=$ & $15 w t \%$ & & & \\
\hline & & & & Flow $=$ & $500 \mathrm{~L} / \mathrm{hr}$ & & & \\
\hline
\end{tabular}




\section{SpinTek II High Shear Rotary Membrane System}

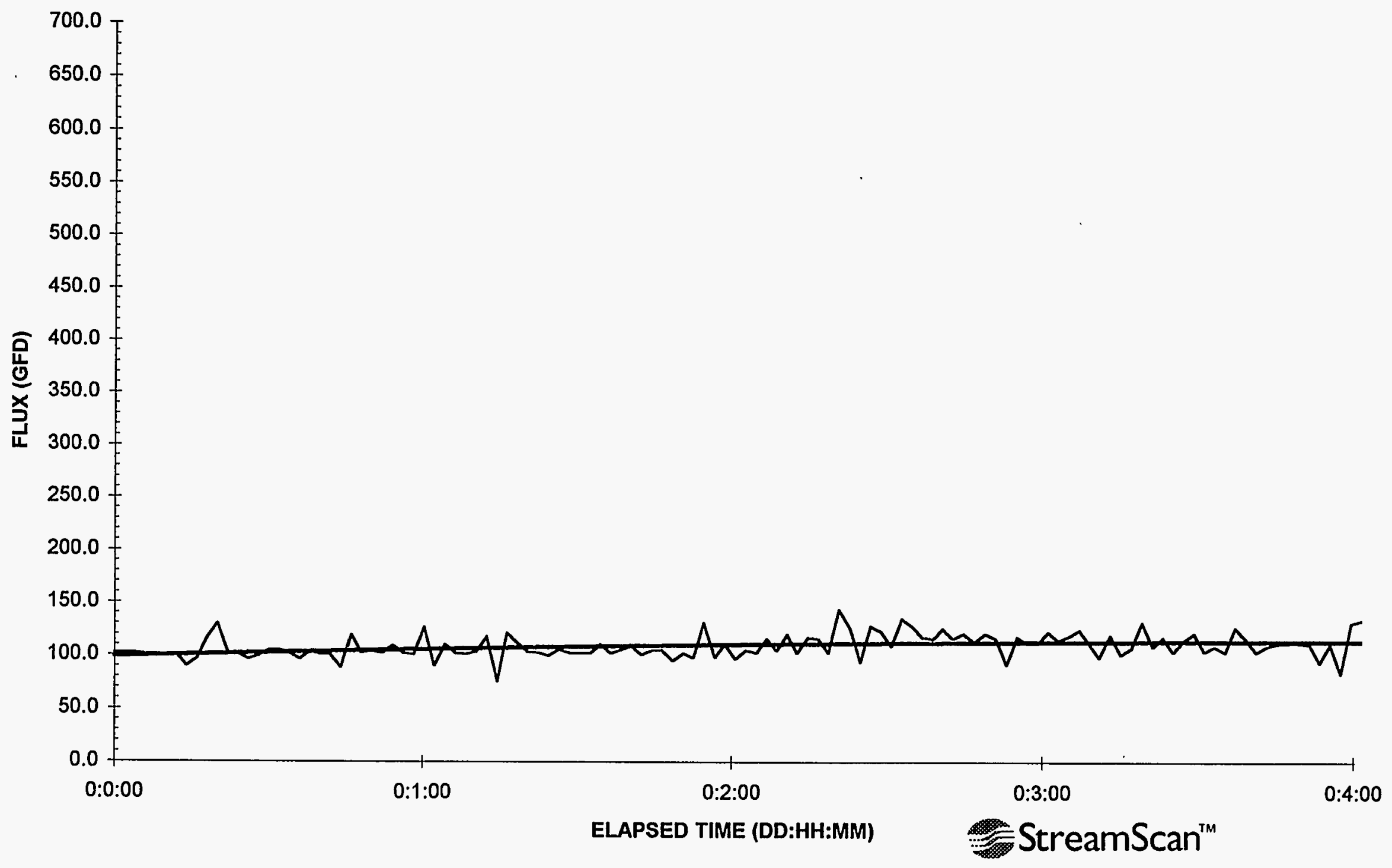




\begin{tabular}{|c|c|c|c|c|c|c|c|c|c|}
\hline $\begin{array}{l}\text { TIME OF } \\
\text { DAY }\end{array}$ & $\begin{array}{c}\text { ELAPSED } \\
\text { TIME }\end{array}$ & $\begin{array}{c}\text { FEED } \\
\text { PRESSURE }\end{array}$ & FEED TEMP & FEED FLOW & $\begin{array}{l}\text { ROTOR } \\
\text { SPEED }\end{array}$ & $\begin{array}{l}\text { PERMEATE } \\
\text { FLOW }\end{array}$ & \begin{tabular}{|c|} 
PERMEATE \\
FLUX
\end{tabular} & & \\
\hline $9: 53$ & $0: 00$ & 59 & 61 & 409 & 1508 & 142 & 101.9 & & \\
\hline $9: 55$ & $0: 02$ & 57 & 61 & 409 & 1503 & 142 & 101.9 & & \\
\hline $9: 57$ & $0: 04$ & 60 & 61 & 408 & 1505 & 142 & 101.9 & & \\
\hline $9: 59$ & $0: 06$ & 53 & 61 & 408 & 1505 & 140 & 100.5 & & \\
\hline $10: 01$ & $0: 08$ & 59 & 61 & 407 & 1514 & 140 & 100.5 & & \\
\hline $10: 03$ & $0: 10$ & 54 & 61 & 407 & 1493 & 138 & 99.1 & & \\
\hline $10: 05$ & $0: 12$ & 62 & 60 & 407 & 1503 & 140 & 100.5 & & \\
\hline $10: 07$ & $0: 14$ & 57 & 61 & 406 & 1511 & 125 & 89.7 & & \\
\hline $10: 09$ & $0: 16$ & 52 & 60 & 406 & 1503 & 135 & 96.9 & & \\
\hline $10: 11$ & $0: 18$ & 67 & 60 & 375 & 1503 & 164 & 117.7 & & \\
\hline $10: 13$ & $0: 20$ & 62 & 61 & 378 & 1501 & 181 & 129.9 & & \\
\hline $10: 15$ & $0: 22$ & 57 & 60 & 405 & 1501 & 143 & 102.6 & & \\
\hline $10: 17$ & $0: 24$ & 58 & 61 & 404 & 1506 & 141 & 101.2 & & \\
\hline $10: 19$ & $0: 26$ & 61 & 61 & 405 & 1504 & 134 & 96.2 & & \\
\hline $10: 21$ & $0: 28$ & 54 & 61 & 404 & 1496 & 139 & 99.8 & & \\
\hline $10: 23$ & $0: 30$ & 52 & 60 & 404 & 1504 & 146 & 104.8 & & \\
\hline $10: 25$ & $0: 32$ & 52 & 60 & 403 & 1504 & 146 & 104.8 & & \\
\hline $10: 27$ & $0: 34$ & 63 & 61 & 403 & 1506 & 142 & 101.9 & & \\
\hline $10: 29$ & $0: 36$ & 52 & 60 & 402 & 1498 & 134 & 96.2 & & \\
\hline $10: 31$ & $0: 38$ & 53 & 60 & 403 & 1507 & 144 & 103.4 & & \\
\hline $10: 34$ & $0: 40$ & 59 & 61 & 402 & 1500 & 141 & 101.2 & & \\
\hline $10: 36$ & $0: 42$ & 53 & 60 & 402 & 1506 & 140 & 100.5 & & \\
\hline $10: 38$ & $0: 44$ & 53 & 60 & 402 & 1501 & 123 & 88.3 & & \\
\hline $10: 40$ & $0: 46$ & 54 & 60 & 402 & 1503 & 166 & 119.2 & & \\
\hline $10: 42$ & $0: 48$ & 62 & 61 & 400 & 1496 & 143 & 102.6 & & \\
\hline $10: 44$ & $0: 50$ & 57 & 61 & 402 & 1501 & 145 & 104.1 & & \\
\hline $10: 46$ & $0: 52$ & 52 & 60 & 400 & 1510 & 142 & 101.9 & & \\
\hline $10: 48$ & $0: 54$ & 52 & 61 & 402 & 1505 & 152 & 109.1 & & \\
\hline $10: 50$ & $0: 56$ & 52 & 61 & 400 & 1506 & 142 & 101.9 & & \\
\hline $10: 52$ & $0: 58$ & 52 & 61 & 402 & 1503 & 140 & 100.5 & & \\
\hline $10: 54$ & $1: 00$ & 54 & 61 & 400 & 1504 & 176 & 126.3 & & \\
\hline $10: 56$ & $1: 02$ & 58 & 61 & 402 & 1494 & 126 & 90.4 & & \\
\hline $10: 58$ & $1: 04$ & 57 & 61 & 401 & 1509 & 153 & 109.8 & & \\
\hline $11: 00$ & $1: 06$ & 54 & 61 & 401 & 1518 & 142 & 101.9 & & \\
\hline $11: 02$ & $1: 08$ & 55 & 60 & 400 & 1504 & 141 & 101.2 & & \\
\hline $11: 04$ & $1: 10$ & 52 & 61 & 400 & 1503 & 144 & 103.4 & & \\
\hline $11: 06$ & $1: 12$ & 69 & 61 & 366 & 1499 & 164 & 117.7 & & \\
\hline $11: 08$ & $1: 14$ & 65 & 61 & 368 & 1506 & 105 & 75.4 & & \\
\hline $11: 10$ & $1: 16$ & 68 & 61 & 366 & 1507 & 168 & 120.6 & & \\
\hline $11: 12$ & $1: 18$ & 67 & 61 & 365 & 1501 & 156 & 112.0 & & \\
\hline $11: 14$ & $1: 20$ & 62 & 61 & 366 & 1509 & 144 & 103.4 & & \\
\hline $11: 16$ & $1: 22$ & 53 & 61 & 387 & 1503 & 143 & 102.6 & & \\
\hline $11: 18$ & $1: 24$ & 61 & 61 & 387 & 1507 & 139 & 99.8 & & \\
\hline $11: 20$ & $1: 26$ & 59 & 61 & 388 & 1512 & 147 & 105.5 & & \\
\hline $11: 22$ & $1: 28$ & 57 & 61 & 387 & 1507 & 143 & 102.6 & & \\
\hline
\end{tabular}


MATR_15.XLS

\begin{tabular}{|c|c|c|c|c|c|c|c|c|}
\hline $\begin{array}{l}\text { TIME OF } \\
\text { DAY }\end{array}$ & $\begin{array}{c}\text { ELAPSED } \\
\text { TIME }\end{array}$ & $\begin{array}{c}\text { FEED } \\
\text { PRESSURE } \\
\end{array}$ & FEED TEMP & FEED FLOW & $\begin{array}{l}\text { ROTOR } \\
\text { SPEED }\end{array}$ & $\begin{array}{c}\text { PERMEATE } \\
\text { FLOW }\end{array}$ & \begin{tabular}{|c|} 
PERMEATE \\
FLUX
\end{tabular} & \\
\hline $11: 24$ & $1: 30$ & 64 & 61 & 387 & 1503 & 142 & 101.9 & \\
\hline $11: 26$ & $1: 32$ & 54 & 61 & 386 & 1494 & 142 & 101.9 & \\
\hline $11: 28$ & $1: 34$ & 55 & 61 & 387 & 1505 & 154 & 110.5 & \\
\hline $11: 30$ & $1: 36$ & 57 & 60 & 387 & 1496 & 142 & 101.9 & \\
\hline $11: 32$ & $1: 38$ & 64 & 61 & 386 & 1506 & 147 & 105.5 & \\
\hline $11: 34$ & $1: 40$ & 57 & 61 & 386 & 1494 & 152 & 109.1 & \\
\hline $11: 36$ & $1: 42$ & 56 & 61 & 387 & 1503 & 140 & 100.5 & \\
\hline $11: 38$ & $1: 44$ & 55 & 61 & 387 & 1504 & 146 & 104.8 & \\
\hline $11: 40$ & $1: 46$ & 64 & 61 & 385 & 1497 & 147 & 105.5 & \\
\hline $11: 42$ & $1: 48$ & 52 & 61 & 385 & 1509 & 132 & 94.8 & \\
\hline $11: 44$ & $1: 50$ & 56 & 61 & 387 & 1495 & 142 & 101.9 & \\
\hline $11: 46$ & $1: 52$ & 55 & 61 & 385 & 1498 & 136 & 97.6 & \\
\hline $11: 48$ & $1: 54$ & 52 & 61 & 386 & 1496 & 182 & 130.6 & Begin Steady-state \\
\hline $11: 50$ & $1: 56$ & 54 & 61 & 385 & 1504 & 137 & 98.3 & \\
\hline $11: 52$ & $1: 58$ & 53 & 61 & 383 & 1498 & 154 & 110.5 & \\
\hline $11: 54$ & $2: 00$ & 58 & 61 & 384 & 1502 & 135 & 96.9 & \\
\hline $11: 56$ & $2: 02$ & 53 & 61 & 385 & 1496 & 146 & 104.8 & \\
\hline $11: 58$ & $2: 04$ & 60 & 61 & 385 & 1503 & 143 & 102.6 & \\
\hline $12: 00$ & $2: 06$ & 55 & 61 & 385 & 1500 & 161 & 115.6 & \\
\hline $12: 02$ & $2: 08$ & 56 & 61 & 383 & 1509 & 146 & 104.8 & \\
\hline $12: 04$ & $2: 10$ & 58 & 61 & 385 & 1504 & 167 & 119.9 & \\
\hline $12: 06$ & $2: 12$ & 56 & 61 & 383 & 1503 & 143 & 102.6 & \\
\hline $12: 08$ & $2: 14$ & 52 & 61 & 383 & 1506 & 163 & 117.0 & \\
\hline $12: 10$ & $2: 17$ & 52 & 61 & 384 & 1496 & 161 & 115.6 & \\
\hline $12: 12$ & $2: 18$ & 56 & 61 & 383 & 1498 & 143 & 102.6 & \\
\hline $12: 14$ & $2: 21$ & 68 & 61 & 304 & 1503 & 200 & 143.6 & \\
\hline $12: 16$ & $2: 23$ & 57 & 61 & 352 & 1501 & 176 & 126.3 & \\
\hline $12: 18$ & $2: 25$ & 62 & 61 & 352 & 1503 & 131 & 94.0 & \\
\hline $12: 20$ & $2: 27$ & 58 & 61 & 352 & 1498 & 178 & 127.8 & \\
\hline $12: 22$ & $2: 29$ & 60 & 61 & 351 & 1498 & 171 & 122.7 & \\
\hline $12: 24$ & $2: 31$ & 57 & 61 & 349 & 1497 & 152 & 109.1 & \\
\hline $12: 26$ & $2: 33$ & 63 & 61 & 350 & 1501 & 188 & 135.0 & \\
\hline $12: 28$ & $2: 35$ & 57 & 61 & 351 & 1503 & 178 & 127.8 & \\
\hline $12: 30$ & $2: 37$ & 59 & 62 & 349 & 1500 & 164 & 117.7 & \\
\hline $12: 32$ & $2: 39$ & 64 & 62 & 349 & 1498 & 161 & 115.6 & \\
\hline $12: 34$ & $2: 41$ & 56 & 62 & 350 & 1496 & 175 & 125.6 & \\
\hline $12: 36$ & $2: 43$ & 56 & 62 & 351 & 1501 & 162 & 116.3 & \\
\hline $12: 38$ & $2: 45$ & 56 & 62 & 349 & 1502 & 168 & 120.6 & \\
\hline $12: 40$ & $2: 47$ & 62 & 61 & 351 & 1500 & 157 & 112.7 & \\
\hline $12: 43$ & $2: 49$ & 57 & 62 & 349 & 1501 & 168 & 120.6 & \\
\hline $12: 45$ & $2: 51$ & 67 & 62 & 350 & 1499 & 162 & 116.3 & \\
\hline $12: 47$ & $2: 53$ & 56 & 62 & 350 & 1502 & 128 & 91.9 & \\
\hline $12: 49$ & $2: 55$ & 64 & 62 & 351 & 1503 & 164 & 117.7 & \\
\hline $12: 51$ & $2: 57$ & 57 & 61 & 349 & 1502 & 155 & 111.3 & \\
\hline $12: 53$ & $2: 59$ & 61 & 62 & 348 & 1498 & 156 & 112.0 & \\
\hline $12: 55$ & $3: 01$ & 59 & 62 & 349 & 1496 & 170 & 122.0 & \\
\hline $12: 57$ & $3: 03$ & 55 & 61 & 349 & 1503 & 159 & 114.1 & \\
\hline $12: 59$ & $3: 05$ & 61 & 61 & 349 & 1498 & 165 & 118.4 & \\
\hline $13: 01$ & $3: 07$ & 67 & 62 & 349 & 1489 & 173 & 124.2 & \\
\hline $13: 03$ & $3: 09$ & 66 & 61 & 349 & 1496 & 156 & 112.0 & \\
\hline
\end{tabular}


MATR_15.XLS

\begin{tabular}{|c|c|c|c|c|c|c|c|c|}
\hline $\begin{array}{l}\text { TIME OF } \\
\text { DAY }\end{array}$ & $\begin{array}{c}\text { ELAPSED } \\
\text { TIME }\end{array}$ & \begin{tabular}{|c|} 
FEED \\
PRESSURE
\end{tabular} & FEED TEMP & FEED FLOW & $\begin{array}{l}\text { ROTOR } \\
\text { SPEED }\end{array}$ & $\begin{array}{c}\text { PERMEATE } \\
\text { FLOW }\end{array}$ & $\begin{array}{c}\text { PERMEATE } \\
\text { FLUX }\end{array}$ & \\
\hline $13: 05$ & $3: 11$ & 63 & 61 & 350 & 1494 & 137 & 98.3 & \\
\hline $13: 07$ & $3: 13$ & 65 & 62 & 350 & 1494 & 166 & 119.2 & \\
\hline $13: 09$ & $3: 15$ & 62 & 62 & 348 & 1502 & 141 & 101.2 & \\
\hline $13: 11$ & $3: 17$ & 68 & 62 & 348 & 1491 & 149 & 107.0 & \\
\hline $13: 13$ & $3: 19$ & 60 & 62 & 349 & 1499 & 183 & 131.4 & \\
\hline $13: 15$ & 3:21 & 64 & 61 & 349 & 1500 & 151 & 108.4 & \\
\hline $13: 17$ & $3: 23$ & 62 & 62 & 347 & 1499 & 163 & 117.0 & \\
\hline $13: 19$ & $3: 25$ & 59 & 62 & 349 & 1504 & 144 & 103.4 & \\
\hline $13: 21$ & $3: 27$ & 68 & 61 & 349 & 1503 & 159 & 114.1 & \\
\hline $13: 23$ & $3: 29$ & 57 & 62 & 347 & 1496 & 168 & 120.6 & \\
\hline $13: 25$ & $3: 31$ & 58 & 61 & 348 & 1493 & 145 & 104.1 & \\
\hline $13: 27$ & $3: 33$ & 64 & 61 & 349 & 1496 & 151 & 108.4 & \\
\hline $13: 29$ & $3: 35$ & 55 & 60 & 347 & 1495 & 144 & 103.4 & \\
\hline $13: 31$ & $3: 37$ & 56 & 61 & 347 & 1495 & 176 & 126.3 & \\
\hline $13: 33$ & $3: 39$ & 56 & 60 & 347 & 1503 & 162 & 116.3 & \\
\hline $13: 35$ & $3: 41$ & 62 & 60 & 350 & 1491 & 144 & 103.4 & \\
\hline $13: 37$ & $3: 43$ & 65 & 61 & 348 & 1501 & 152 & 109.1 & \\
\hline $13: 39$ & $3: 45$ & 57 & 61 & 347 & 1495 & 155 & 111.3 & \\
\hline $13: 41$ & $3: 47$ & 59 & 61 & 349 & 1494 & 156 & 112.0 & \\
\hline $13: 43$ & $3: 49$ & 68 & 60 & 350 & 1501 & 156 & 112.0 & \\
\hline $13: 45$ & $3: 51$ & 58 & 61 & 348 & 1496 & 154 & 110.5 & \\
\hline $13: 47$ & $3: 53$ & 62 & 60 & 348 & 1502 & 130 & 93.3 & \\
\hline $13: 49$ & $3: 55$ & 55 & 61 & 348 & 1497 & 154 & 110.5 & \\
\hline $13: 51$ & $3: 57$ & 54 & 60 & 346 & 1497 & 116 & 83.3 & \\
\hline $13: 53$ & $3: 59$ & 55 & 61 & 348 & 1491 & 182 & 130.6 & \\
\hline $13: 55$ & $4: 01$ & 63 & 60 & 347 & 1504 & 186 & 133.5 & End Steady-state \\
\hline \multicolumn{2}{|c|}{ Steady-state Avg. } & 59 & 61 & 355 & 1499 & 158 & 114 & \\
\hline \multicolumn{2}{|l|}{ Maximum } & 68 & 62 & 386 & 1509 & 200 & 144 & \\
\hline Minimum & & 52 & 60 & 304 & 1489 & 116 & 83 & \\
\hline \multicolumn{2}{|c|}{ Standard Deviation } & 4.4 & 0.6 & 15.7 & 4.0 & 16.0 & 11.5 & \\
\hline \multicolumn{4}{|c|}{ TEST CONDITIONS: MATR_15.XLS } & Temp. $=t$ & $60^{\circ} \mathrm{F}$ & & & \\
\hline & & & & Press. $=$ & 60 psig & & & \\
\hline \multicolumn{3}{|c|}{ Date Run: 16-May-96 } & & Rotor $=$ & $1500 \mathrm{rpm}$ & & & \\
\hline & & & & Solids $=$ & $30 w t \%$ & & & \\
\hline & & & & Flow $=$ & $400 \mathrm{~L} / \mathrm{hr}$ & & & \\
\hline
\end{tabular}




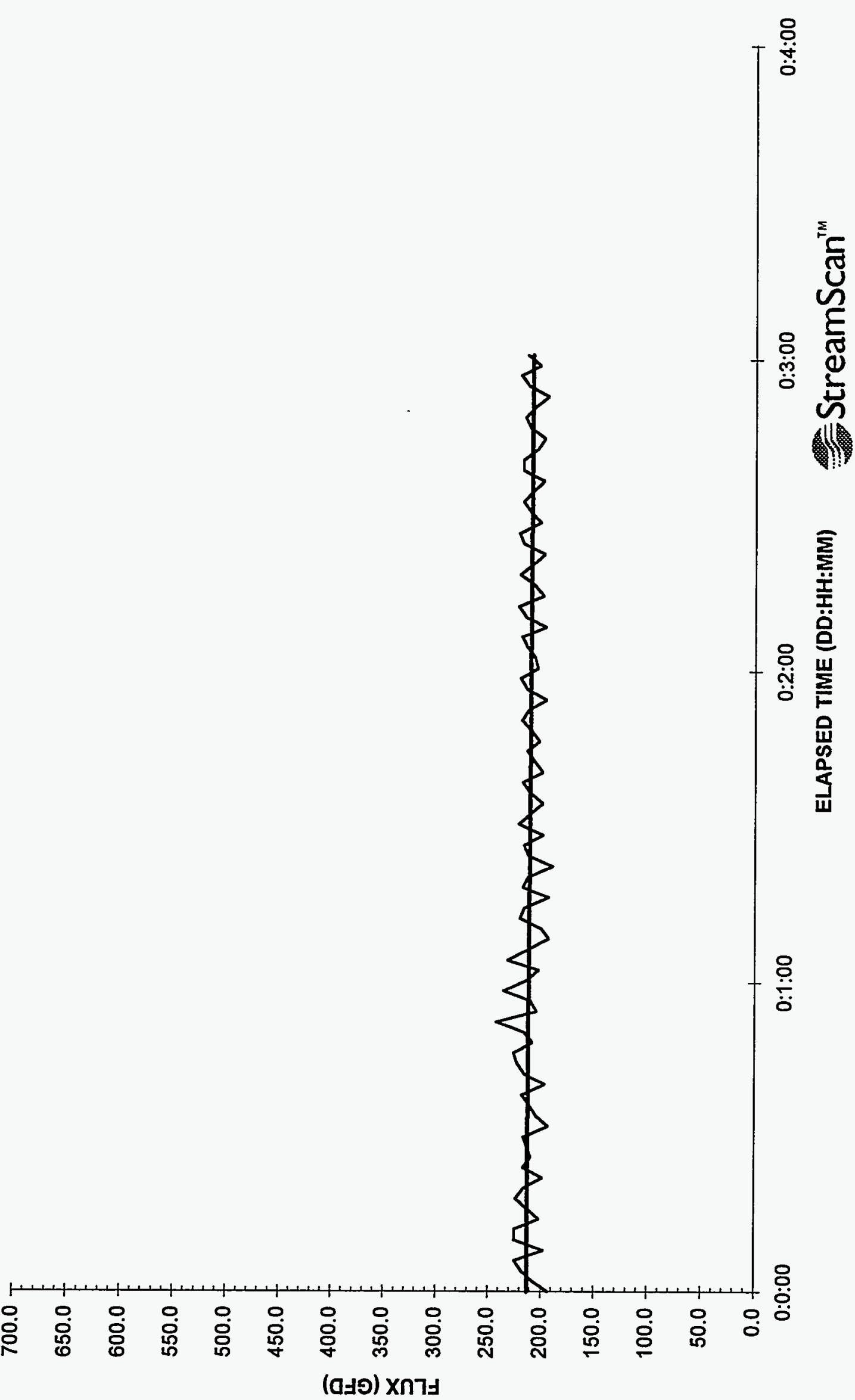




\begin{tabular}{|c|c|c|c|c|c|c|c|c|}
\hline $\begin{array}{l}\text { TIME OF } \\
\text { DAY }\end{array}$ & $\begin{array}{l}\text { ELAPSED } \\
\text { TIME }\end{array}$ & $\begin{array}{c}\text { FEED } \\
\text { PRESSURE }\end{array}$ & FEED TEMP & FEED FLOW & $\begin{array}{l}\text { ROTOR } \\
\text { SPEED }\end{array}$ & $\begin{array}{c}\text { PERMEATE } \\
\text { FLOW }\end{array}$ & \begin{tabular}{|c|} 
PERMEATE \\
FLUX
\end{tabular} & \\
\hline $15: 15$ & $0: 00$ & 59 & 90 & 359 & 1511 & 269 & 193.1 & \\
\hline $15: 17$ & $0: 02$ & 65 & 89 & 368 & 1516 & 289 & 207.5 & \\
\hline $15: 19$ & $0: 04$ & 66 & 89 & 377 & 1517 & 304 & 218.2 & \\
\hline $15: 21$ & $0: 06$ & 65 & 90 & 363 & 1518 & 313 & 224.7 & \\
\hline $15: 23$ & $0: 08$ & 61 & 90 & 368 & 1515 & 275 & 197.4 & \\
\hline $15: 25$ & $0: 10$ & 60 & 89 & 358 & 1516 & 314 & 225.4 & \\
\hline $15: 27$ & $0: 12$ & 65 & 90 & 373 & 1517 & 313 & 224.7 & \\
\hline $15: 29$ & $0: 14$ & 60 & 90 & 363 & 1513 & 281 & 201.7 & \\
\hline $15: 31$ & $0: 16$ & 59 & 89 & 358 & 1503 & 297 & 213.2 & \\
\hline $15: 33$ & $0: 18$ & 65 & 89 & 374 & 1510 & 312 & 224.0 & \\
\hline $15: 35$ & $0: 20$ & 66 & 90 & 361 & 1507 & 301 & 216.1 & \\
\hline $15: 37$ & $0: 22$ & 69 & 90 & 359 & 1505 & 277 & 198.8 & \\
\hline $15: 39$ & $0: 24$ & 65 & 88 & 365 & 1505 & 302 & 216.8 & \\
\hline $15: 41$ & $0: 26$ & 63 & 90 & 370 & 1516 & 292 & 209.6 & \\
\hline $15: 43$ & $0: 28$ & 70 & 90 & 360 & 1512 & 297 & 213.2 & \\
\hline $15: 45$ & $0: 30$ & 59 & 91 & 369 & 1511 & 302 & 216.8 & \\
\hline $15: 47$ & $0: 32$ & 65 & 91 & 368 & 1512 & 269 & 193.1 & \\
\hline $15: 49$ & $0: 34$ & 58 & 89 & 366 & 1511 & 285 & 204.6 & \\
\hline $15: 51$ & $0: 36$ & 58 & 89 & 374 & 1507 & 294 & 211.0 & \\
\hline $15: 53$ & $0: 38$ & 64 & 90 & 370 & 1516 & 304 & 218.2 & \\
\hline $15: 55$ & $0: 40$ & 62 & 90 & 358 & 1508 & 274 & 196.7 & \\
\hline $15: 57$ & $0: 42$ & 63 & 89 & 377 & 1514 & 300 & 215.3 & \\
\hline $15: 59$ & $0: 44$ & 63 & 89 & 366 & 1517 & 310 & 222.5 & \\
\hline $16: 01$ & $0: 46$ & 59 & 91 & 359 & 1513 & 315 & 226.1 & \\
\hline $16: 03$ & $0: 48$ & 60 & 90 & 367 & 1510 & 290 & 208.2 & \\
\hline $16: 05$ & $0: 50$ & 61 & 89 & 386 & 1517 & 301 & 216.1 & \\
\hline $16: 07$ & $0: 52$ & 64 & 90 & 370 & 1519 & 338 & 242.6 & \\
\hline $16: 09$ & $0: 54$ & 63 & 90 & 374 & 1522 & 285 & 204.6 & \\
\hline $16: 11$ & $0: 56$ & 67 & 89 & 389 & 1515 & 293 & 210.3 & \\
\hline $16: 14$ & $0: 58$ & 63 & 90 & 378 & 1517 & 328 & 235.4 & \\
\hline $16: 16$ & $1: 00$ & 64 & 91 & 374 & 1510 & 298 & 213.9 & \\
\hline $16: 18$ & $1: 02$ & 60 & 90 & 363 & 1513 & 282 & 202.4 & \\
\hline $16: 20$ & $1: 04$ & 61 & 89 & 373 & 1508 & 323 & 231.9 & \\
\hline $16: 22$ & $1: 06$ & 57 & 90 & 391 & 1510 & 297 & 213.2 & Begin Steady-state \\
\hline $16: 24$ & $1: 08$ & 61 & 91 & 391 & 1520 & 269 & 193.1 & \\
\hline $16: 26$ & $1: 10$ & 59 & 89 & 400 & 1512 & 279 & 200.3 & \\
\hline $16: 28$ & $1: 12$ & 55 & 89 & 374 & 1514 & 307 & 220.4 & \\
\hline $16: 30$ & $1: 14$ & 59 & 90 & 397 & 1513 & 301 & 216.1 & \\
\hline $16: 32$ & $1: 16$ & 62 & 90 & 388 & 1500 & 269 & 193.1 & \\
\hline $16: 34$ & $1: 18$ & 55 & 89 & 394 & 1503 & 303 & 217.5 & \\
\hline $16: 36$ & $1: 20$ & 56 & 90 & 402 & 1513 & 297 & 213.2 & \\
\hline $16: 38$ & $1: 22$ & 61 & 90 & 374 & 1515 & 264 & 189.5 & \\
\hline $16: 40$ & $1: 24$ & 59 & 89 & 392 & 1514 & 296 & 212.5 & \\
\hline $16: 42$ & $1: 26$ & 65 & 90 & 400 & 1513 & 302 & 216.8 & \\
\hline $16: 44$ & $1: 28$ & 55 & 90 & 396 & 1512 & 277 & 198.8 & \\
\hline
\end{tabular}


MATR_16.XLS

\begin{tabular}{|c|c|c|c|c|c|c|c|c|}
\hline $\begin{array}{c}\text { TIME OF } \\
\text { DAY }\end{array}$ & $\begin{array}{l}\text { ELAPSED } \\
\text { TIME }\end{array}$ & $\begin{array}{c}\text { FEED } \\
\text { PRESSURE } \\
\end{array}$ & FEED TEMP & FEED FLOW & $\begin{array}{l}\text { ROTOR } \\
\text { SPEED } \\
\end{array}$ & $\begin{array}{c}\text { PERMEATE } \\
\text { FLOW }\end{array}$ & $\begin{array}{l}\text { PERMEATE } \\
\text { FLUX }\end{array}$ & \\
\hline $16: 46$ & $1: 30$ & 59 & 89 & 400 & 1514 & 309 & 221.8 & \\
\hline $16: 48$ & $1: 32$ & 63 & 89 & 397 & 1517 & 294 & 211.0 & \\
\hline $16: 50$ & $1: 34$ & 55 & 91 & 389 & 1503 & 278 & 199.6 & \\
\hline $16: 52$ & $1: 36$ & 57 & 89 & 382 & 1504 & 294 & 211.0 & \\
\hline $16: 54$ & $1: 38$ & 56 & 89 & 393 & 1502 & 304 & 218.2 & \\
\hline $16: 56$ & $1: 40$ & 64 & 91 & 382 & 1518 & 278 & 199.6 & \\
\hline $16: 58$ & $1: 42$ & 60 & 90 & 386 & 1515 & 288 & 206.7 & \\
\hline $17: 00$ & $1: 44$ & 63 & 90 & 395 & 1514 & 298 & 213.9 & \\
\hline $17: 02$ & $1: 46$ & 63 & 91 & 384 & 1515 & 282 & 202.4 & \\
\hline $17: 04$ & $1: 48$ & 64 & 90 & 400 & 1507 & 293 & 210.3 & \\
\hline $17: 06$ & $1: 50$ & 60 & 89 & 391 & 1523 & 305 & 218.9 & \\
\hline $17: 08$ & $1: 52$ & 55 & 91 & 399 & 1515 & 296 & 212.5 & \\
\hline $17: 10$ & $1: 54$ & 57 & 90 & 394 & 1512 & 273 & 196.0 & \\
\hline $17: 12$ & $1: 56$ & 57 & 89 & 388 & 1510 & 298 & 213.9 & \\
\hline $17: 14$ & $1: 58$ & 59 & 90 & 400 & 1506 & 307 & 220.4 & \\
\hline $17: 16$ & $2: 00$ & 65 & 91 & 400 & 1517 & 284 & 203.9 & \\
\hline $17: 18$ & $2: 02$ & 59 & 90 & 391 & 1512 & 288 & 206.7 & \\
\hline $17: 20$ & $2: 04$ & 54 & 89 & 389 & 1517 & 298 & 213.9 & \\
\hline $17: 22$ & $2: 06$ & 57 & 91 & 386 & 1509 & 305 & 218.9 & \\
\hline $17: 24$ & $2: 08$ & 56 & 90 & 392 & 1512 & 274 & 196.7 & \\
\hline $17: 26$ & $2: 10$ & 55 & 89 & 389 & 1516 & 300 & 215.3 & \\
\hline $17: 28$ & $2: 12$ & 65 & 90 & 401 & 1514 & 310 & 222.5 & \\
\hline $17: 30$ & $2: 14$ & 60 & 90 & 388 & 1521 & 277 & 198.8 & \\
\hline $17: 32$ & $2: 16$ & $\overline{64}$ & 89 & 382 & 1510 & 289 & 207.5 & \\
\hline $17: 34$ & $2: 18$ & 62 & 90 & 395 & 1512 & 308 & 221.1 & \\
\hline $17: 36$ & $2: 20$ & 55 & 91 & 393 & 1511 & 291 & 208.9 & \\
\hline $17: 38$ & $2: 22$ & 64 & 90 & 398 & 1519 & 276 & 198.1 & \\
\hline $17: 40$ & $2: 24$ & 60 & 89 & 389 & 1503 & 303 & 217.5 & \\
\hline $17: 42$ & $2: 26$ & 60 & 90 & 390 & 1510 & 309 & 221.8 & \\
\hline $17: 44$ & $2: 28$ & 60 & 90 & 395 & 1513 & 281 & 201.7 & \\
\hline $17: 46$ & $2: 30$ & 54 & 89 & 397 & 1507 & 294 & 211.0 & \\
\hline $17: 48$ & $2: 32$ & 57 & 89 & 400 & 1519 & 304 & 218.2 & \\
\hline $17: 50$ & $2: 34$ & 60 & 91 & 390 & 1510 & 291 & 208.9 & \\
\hline $17: 52$ & $2: 36$ & 55 & 90 & 383 & 1515 & 277 & 198.8 & \\
\hline $17: 54$ & $2: 38$ & 56 & 89 & 380 & 1517 & 304 & 218.2 & \\
\hline $17: 56$ & $2: 40$ & 56 & 91 & 390 & 1507 & 304 & 218.2 & \\
\hline $17: 58$ & $2: 43$ & 59 & 91 & 390 & 1512 & 285 & 204.6 & \\
\hline $18: 00$ & $2: 45$ & 56 & 91 & 388 & 1516 & 276 & 198.1 & \\
\hline $18: 02$ & $2: 47$ & 56 & 89 & 392 & 1515 & 295 & 211.8 & \\
\hline $18: 04$ & $2: 49$ & 58 & 90 & 397 & 1517 & 301 & 216.1 & \\
\hline $18: 06$ & $2: 51$ & 57 & 91 & 376 & 1515 & 289 & 207.5 & \\
\hline $18: 08$ & $2: 53$ & 57 & 90 & 386 & 1512 & 271 & 194.5 & \\
\hline $18: 10$ & $2: 55$ & 61 & 89 & 396 & 1517 & 297 & 213.2 & \\
\hline $18: 12$ & $2: 57$ & 58 & 90 & 392 & 1509 & 307 & 220.4 & \\
\hline $18: 14$ & $2: 59$ & 58 & 90 & 380 & 1512 & 282 & 202.4 & \\
\hline $18: 16$ & $3: 01$ & 62 & 89 & 397 & 1513 & 298 & 213.9 & End Steady-state \\
\hline
\end{tabular}


MATR_16.XLS

\begin{tabular}{|c|c|c|c|c|c|c|c|c|c|}
\hline $\begin{array}{c}\text { TIME OF } \\
\text { DAY }\end{array}$ & $\begin{array}{c}\text { ELAPSED } \\
\text { TIME }\end{array}$ & $\begin{array}{c}\text { FEED } \\
\text { PRESSURE }\end{array}$ & FEED TEMP & FEED FLOW & $\begin{array}{l}\text { ROTOR } \\
\text { SPEED }\end{array}$ & $\begin{array}{c}\text { PERMEATE } \\
\text { FLOW }\end{array}$ & \begin{tabular}{|c}
$\begin{array}{c}\text { PERMEATE } \\
\text { FLUX }\end{array}$ \\
\end{tabular} & & \\
\hline \multicolumn{2}{|c|}{ Steady-state Avg. } & 59 & 90 & 391 & 1512 & 292 & 209 & & \\
\hline \multicolumn{2}{|l|}{ Maximum } & 65 & 91 & 402 & 1523 & 310 & 223 & & \\
\hline \multicolumn{2}{|l|}{ Minimum } & 54 & 89 & 374 & 1500 & 264 & 190 & & \\
\hline \multicolumn{2}{|c|}{ Standard Deviation } & 3.2 & 0.8 & 6.9 & 4.9 & 12.4 & 8.9 & & \\
\hline & & & & & & & & & \\
\hline \multicolumn{4}{|c|}{ TEST CONDITIONS: MATR_16.XLS } & Temp. $=$ & $90^{\circ} \mathrm{F}$ & & & & \\
\hline & & & & Press. $=$ & 60 psig & & & & \\
\hline \multicolumn{3}{|c|}{ Date Run: 16-May-96 } & & Rotor $=$ & $1500 \mathrm{rpm}$ & & & & \\
\hline & & & & Solids $=$ & $15 w t \%$ & & & & \\
\hline & & & & Flow $=$ & $400 \mathrm{~L} / \mathrm{hr}$ & & & & \\
\hline
\end{tabular}




\section{SpinTek II High Shear Rotary Membrane System}

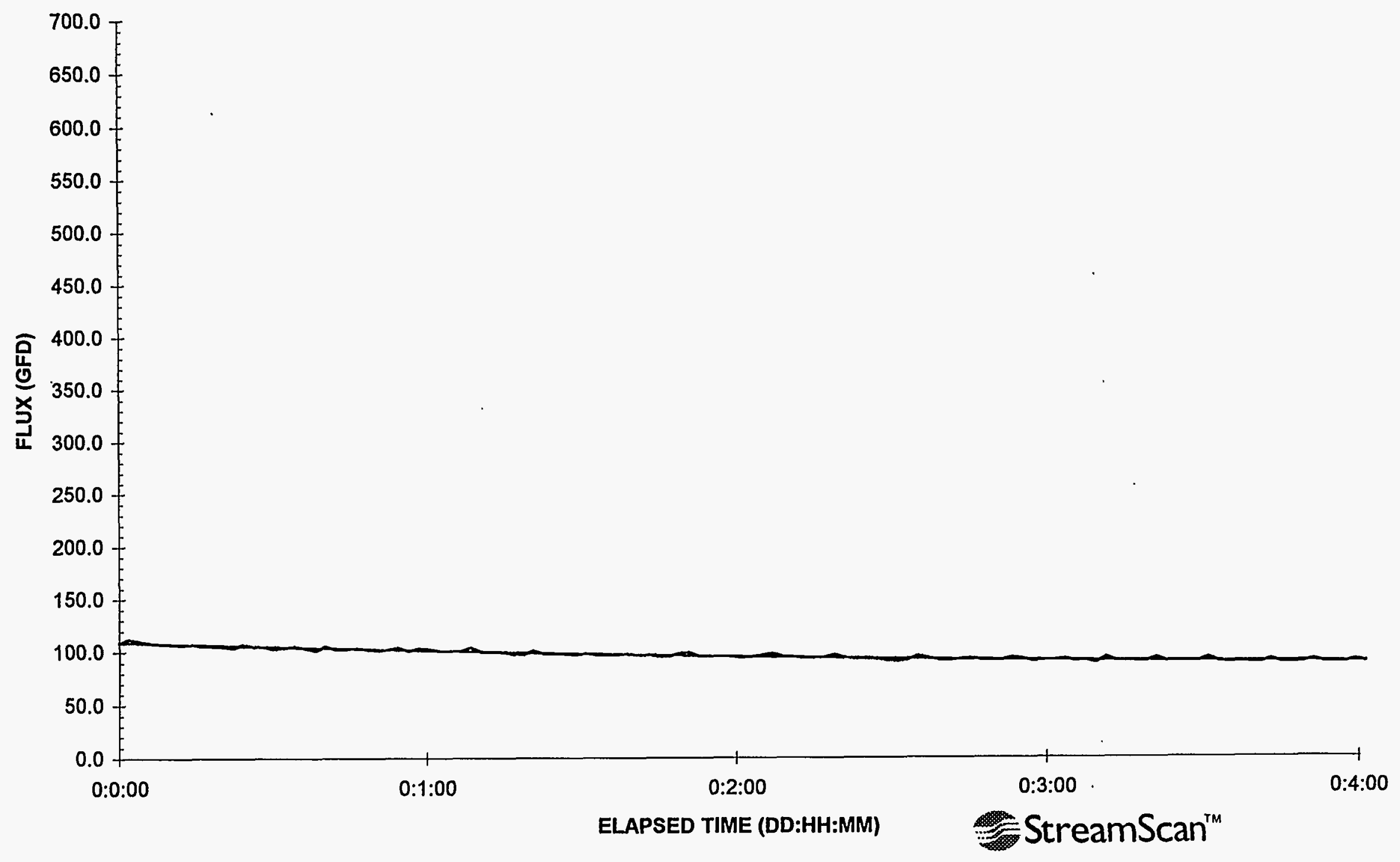




\begin{tabular}{|c|c|c|c|c|c|c|c|c|c|}
\hline $\begin{array}{l}\text { TIME OF } \\
\text { DAY }\end{array}$ & $\begin{array}{c}\text { ELAPSED } \\
\text { TIME }\end{array}$ & $\begin{array}{c}\text { FEED } \\
\text { PRESSURE }\end{array}$ & FEED TEMP & FEED FLOW & $\begin{array}{l}\text { ROTOR } \\
\text { SPEED }\end{array}$ & $\begin{array}{c}\text { PERMEATE } \\
\text { FLOW }\end{array}$ & \begin{tabular}{|c|}
$\begin{array}{c}\text { PERMEATE } \\
\text { FLUX }\end{array}$ \\
\end{tabular} & & \\
\hline $20: 44$ & $0: 00$ & 62 & 62 & 326 & 997 & 152 & 109.1 & & \\
\hline $20: 46$ & $0: 02$ & 66 & 62 & 306 & 998 & 156 & 112.0 & & \\
\hline $20: 48$ & $0: 04$ & 55 & 61 & 304 & 995 & 153 & 109.8 & & \\
\hline $20: 50$ & $0: 06$ & 57 & 61 & 309 & 996 & 151 & 108.4 & & \\
\hline $20: 52$ & $0: 08$ & 68 & 60 & 307 & 994 & 150 & 107.7 & & \\
\hline $20: 54$ & $0: 10$ & 58 & 60 & 306 & 996 & 149 & 107.0 & & \\
\hline $20: 57$ & $0: 12$ & 61 & 60 & 305 & 1005 & 148 & 106.2 & & \\
\hline $20: 59$ & $0: 14$ & 53 & 60 & 302 & 994 & 149 & 107.0 & & \\
\hline $21: 01$ & $0: 16$ & 68 & 60 & 303 & 995 & 147 & 105.5 & & \\
\hline $21: 03$ & $0: 18$ & 59 & 60 & 301 & 1002 & 147 & 105.5 & & \\
\hline $21: 05$ & $0: 20$ & 66 & 61 & 302 & 996 & 146 & 104.8 & & \\
\hline $21: 07$ & $0: 22$ & 61 & 60 & 305 & 1004 & 144 & 103.4 & & \\
\hline $21: 09$ & $0: 24$ & 56 & 60 & 296 & 999 & 149 & 107.0 & & \\
\hline $21: 11$ & $0: 26$ & 60 & 60 & 304 & 997 & 147 & 105.5 & & \\
\hline $21: 13$ & $0: 28$ & 58 & 61 & 310 & 996 & 146 & 104.8 & & \\
\hline $21: 15$ & $0: 30$ & 53 & 60 & 299 & 994 & 143 & 102.6 & & \\
\hline $21: 17$ & $0: 32$ & 58 & 60 & 300 & 995 & 145 & 104.1 & & \\
\hline $21: 19$ & $0: 34$ & 59 & 61 & 303 & 994 & 147 & 105.5 & & \\
\hline $21: 21$ & $0: 36$ & 61 & 60 & 297 & 993 & 144 & 103.4 & & \\
\hline $21: 23$ & $0: 38$ & 60 & 60 & 299 & 995 & 141 & 101.2 & & \\
\hline $21: 25$ & $0: 40$ & 57 & 60 & 298 & 996 & 147 & 105.5 & & \\
\hline $21: 27$ & $0: 42$ & 57 & 60 & 305 & 999 & 142 & 101.9 & & \\
\hline $21: 29$ & $0: 44$ & 57 & 60 & 303 & 994 & 143 & 102.6 & & \\
\hline $21: 31$ & $0: 46$ & 61 & 61 & 304 & 992 & 144 & 103.4 & & \\
\hline $21: 33$ & $0: 48$ & 62 & 61 & 293 & 997 & 142 & 101.9 & & \\
\hline $21: 35$ & $0: 50$ & 58 & 60 & 306 & 997 & 141 & 101.2 & & \\
\hline $21: 37$ & $0: 52$ & 54 & 61 & 293 & 996 & 142 & 101.9 & & \\
\hline $21: 39$ & $0: 54$ & 58 & 60 & 290 & 994 & 145 & 104.1 & & \\
\hline $21: 41$ & $0: 56$ & 52 & 60 & 292 & 992 & 140 & 100.5 & & \\
\hline $21: 43$ & $0: 58$ & 59 & 61 & 301 & 997 & 144 & 103.4 & & \\
\hline $21: 45$ & $1: 00$ & 60 & 60 & 298 & 997 & 143 & 102.6 & & \\
\hline $21: 47$ & $1: 02$ & 61 & 61 & 296 & 1001 & 140 & 100.5 & & \\
\hline $21: 49$ & $1: 04$ & 53 & 60 & 285 & 995 & 141 & 101.2 & & \\
\hline $21: 51$ & $1: 06$ & 54 & 60 & 307 & 993 & 140 & 100.5 & & \\
\hline $21: 53$ & $1: 08$ & 61 & 60 & 307 & 999 & 145 & 104.1 & & \\
\hline $21: 55$ & $1: 10$ & 54 & 61 & 296 & 997 & 139 & 99.8 & & \\
\hline $21: 57$ & $1: 12$ & 53 & 61 & 309 & 993 & 138 & 99.1 & & \\
\hline $21: 59$ & $1: 14$ & 58 & 61 & 292 & 999 & 138 & 99.1 & & \\
\hline $22: 01$ & $1: 16$ & 53 & 60 & 298 & 995 & 136 & 97.6 & & \\
\hline $22: 03$ & $1: 18$ & 55 & 60 & 297 & 1004 & 135 & 96.9 & & \\
\hline $22: 05$ & $1: 20$ & 67 & 60 & 292 & 998 & 141 & 101.2 & & \\
\hline $22: 07$ & $1: 22$ & 60 & 61 & 292 & 1005 & 137 & 98.3 & & \\
\hline $22: 09$ & $1: 24$ & 53 & 60 & 309 & 993 & 136 & 97.6 & & \\
\hline $22: 11$ & $1: 26$ & 59 & 61 & 293 & 993 & 136 & 97.6 & & \\
\hline $22: 13$ & $1: 28$ & 52 & 60 & 297 & 994 & 135 & 96.9 & & \\
\hline
\end{tabular}


MATR_17.XLS

\begin{tabular}{|c|c|c|c|c|c|c|c|c|}
\hline $\begin{array}{c}\text { TIME OF } \\
\text { DAY }\end{array}$ & $\begin{array}{l}\text { ELAPSED } \\
\text { TIME }\end{array}$ & $\begin{array}{c}\text { FEED } \\
\text { PRESSURE }\end{array}$ & FEED TEMP & FEED FLOW & $\begin{array}{l}\text { ROTOR } \\
\text { SPEED }\end{array}$ & $\begin{array}{c}\text { PERMEATE } \\
\text { FLOW }\end{array}$ & \begin{tabular}{|c|}
$\begin{array}{c}\text { PERMEATE } \\
\text { FLUX }\end{array}$ \\
\end{tabular} & \\
\hline $22: 15$ & $1: 30$ & 54 & 60 & 308 & 997 & 137 & 98.3 & \\
\hline $22: 17$ & $1: 32$ & 61 & 61 & 303 & 997 & 135 & 96.9 & \\
\hline $22: 19$ & $1: 34$ & 67 & 60 & 298 & 996 & 134 & 96.2 & \\
\hline $22: 21$ & $1: 36$ & 59 & 61 & 305 & 994 & 134 & 96.2 & \\
\hline $22: 23$ & $1: 38$ & 57 & 60 & 295 & 994 & 136 & 97.6 & \\
\hline $22: 25$ & $1: 40$ & 54 & 61 & 311 & 996 & 134 & 96.2 & \\
\hline $22: 27$ & $1: 42$ & 56 & 60 & 305 & 1005 & 135 & 96.9 & \\
\hline $22: 29$ & $1: 44$ & 64 & 60 & 311 & 1000 & 133 & 95.5 & \\
\hline $22: 31$ & $1: 46$ & 56 & 60 & 296 & 997 & 132 & 94.8 & \\
\hline $22: 33$ & $1: 48$ & 56 & 60 & 305 & 1001 & 137 & 98.3 & \\
\hline $22: 35$ & $1: 50$ & 52 & 60 & 303 & 994 & 138 & 99.1 & \\
\hline $22: 37$ & $1: 52$ & 55 & 61 & 305 & 997 & 133 & 95.5 & \\
\hline $22: 39$ & $1: 54$ & 58 & 61 & 308 & 995 & 133 & 95.5 & \\
\hline $22: 41$ & $1: 56$ & 65 & 60 & 295 & 995 & 133 & 95.5 & \\
\hline $22: 43$ & $1: 58$ & 67 & 61 & 296 & 1001 & 133 & 95.5 & \\
\hline $22: 45$ & $2: 00$ & 57 & 60 & 293 & 996 & 131 & 94.0 & Begin Steady-state \\
\hline $22: 47$ & $2: 02$ & 53 & 60 & 297 & 995 & 132 & 94.8 & \\
\hline $22: 49$ & $2: 04$ & 57 & 60 & 296 & 993 & 134 & 96.2 & \\
\hline $22: 51$ & $2: 06$ & 54 & 60 & 296 & 997 & 137 & 98.3 & \\
\hline $22: 53$ & $2: 08$ & 57 & 61 & 303 & 997 & 132 & 94.8 & \\
\hline $22: 55$ & $2: 10$ & 57 & 61 & 308 & 998 & 132 & 94.8 & \\
\hline $22: 57$ & $2: 12$ & 53 & 60 & 299 & 998 & 130 & 93.3 & \\
\hline $22: 59$ & $2: 14$ & 60 & 61 & 303 & 997 & 131 & 94.0 & \\
\hline $23: 01$ & $2: 17$ & 67 & 60 & 290 & 996 & 131 & 94.0 & \\
\hline $23: 04$ & $2: 19$ & 59 & 59 & 302 & 1001 & 135 & 96.9 & \\
\hline $23: 06$ & $2: 21$ & 61 & 61 & 298 & 1004 & 131 & 94.0 & \\
\hline $23: 08$ & $2: 23$ & 54 & 61 & 295 & 999 & 129 & 92.6 & \\
\hline $23: 10$ & $2: 25$ & 54 & 60 & 299 & 1000 & 129 & 92.6 & \\
\hline $23: 12$ & $2: 27$ & 59 & 60 & 301 & 998 & 129 & 92.6 & \\
\hline $23: 14$ & $2: 29$ & 66 & 60 & 300 & 997 & 127 & 91.2 & \\
\hline $23: 16$ & $2: 31$ & 53 & 60 & 292 & 995 & 126 & 90.4 & \\
\hline $23: 18$ & $2: 33$ & 60 & 60 & 298 & 1008 & 128 & 91.9 & \\
\hline $23: 20$ & $2: 35$ & 59 & 60 & 290 & 997 & 133 & 95.5 & \\
\hline $23: 22$ & $2: 37$ & 66 & 61 & 305 & 996 & 130 & 93.3 & \\
\hline $23: 24$ & $2: 39$ & 59 & 61 & 306 & 994 & 128 & 91.9 & \\
\hline $23: 26$ & $2: 41$ & 66 & 60 & 280 & 994 & 127 & 91.2 & \\
\hline $23: 28$ & $2: 43$ & 52 & 60 & 300 & 1011 & 129 & 92.6 & \\
\hline $23: 30$ & $2: 45$ & 57 & 60 & 299 & 997 & 130 & 93.3 & \\
\hline $23: 32$ & $2: 47$ & 55 & 60 & 297 & 997 & 128 & 91.9 & \\
\hline $23: 34$ & $2: 49$ & 52 & 60 & 303 & 996 & 127 & 91.2 & \\
\hline $23: 36$ & $2: 51$ & 53 & 60 & 292 & 994 & 127 & 91.2 & \\
\hline $23: 38$ & $2: 53$ & 55 & 60 & 297 & 998 & 131 & 94.0 & \\
\hline $23: 40$ & $2: 55$ & 56 & 60 & 303 & 997 & 129 & 92.6 & \\
\hline $23: 42$ & $2: 57$ & 54 & 60 & 283 & 994 & 126 & 90.4 & \\
\hline $23: 44$ & $2: 59$ & 58 & 60 & 298 & 996 & 127 & 91.2 & \\
\hline $23: 46$ & $3: 01$ & 60 & 60 & 296 & 994 & 127 & 91.2 & \\
\hline $23: 48$ & $3: 03$ & 51 & 60 & 296 & 999 & 129 & 92.6 & \\
\hline $23: 50$ & $3: 05$ & 50 & 61 & 296 & 996 & 126 & 90.4 & \\
\hline $23: 52$ & $3: 07$ & 53 & 60 & 303 & 998 & 127 & 91.2 & \\
\hline $23: 54$ & $3: 09$ & 66 & 60 & 296 & 995 & 124 & 89.0 & \\
\hline
\end{tabular}


MATR_17.XLS

\begin{tabular}{|c|c|c|c|c|c|c|c|c|}
\hline $\begin{array}{c}\text { TIME OF } \\
\text { DAY }\end{array}$ & $\begin{array}{c}\text { ELAPSED } \\
\text { TIME }\end{array}$ & $\begin{array}{c}\text { FEED } \\
\text { PRESSURE }\end{array}$ & FEED TEMP & FEED FLOW & $\begin{array}{l}\text { ROTOR } \\
\text { SPEED } \\
\end{array}$ & \begin{tabular}{|c|}
$\begin{array}{c}\text { PERMEATE } \\
\text { FLOW }\end{array}$ \\
\end{tabular} & $\begin{array}{c}\text { PERMEATE } \\
\text { FLUX }\end{array}$ & \\
\hline $23: 56$ & $3: 11$ & 51 & 60 & 292 & 996 & 131 & 94.0 & \\
\hline $23: 58$ & $3: 13$ & 62 & 60 & 304 & 994 & 126 & 90.4 & \\
\hline $0: 00$ & $3: 15$ & 54 & 61 & 288 & 996 & 126 & 90.4 & \\
\hline $0: 02$ & $3: 17$ & 53 & 61 & 295 & 994 & 126 & 90.4 & \\
\hline $0: 04$ & $3: 19$ & 54 & 60 & 291 & 993 & 125 & 89.7 & \\
\hline $0: 06$ & $3: 21$ & 56 & 60 & 283 & 995 & 130 & 93.3 & \\
\hline $0: 08$ & $3: 23$ & 59 & 61 & 301 & 997 & 125 & 89.7 & \\
\hline $0: 10$ & $3: 25$ & 59 & 60 & 296 & 995 & 126 & 90.4 & \\
\hline $0: 12$ & $3: 27$ & 55 & 60 & 308 & 995 & 125 & 89.7 & \\
\hline $0: 14$ & $3: 29$ & 64 & 60 & 299 & 995 & 125 & 89.7 & \\
\hline $0: 16$ & $3: 31$ & 58 & 60 & 305 & 1000 & 130 & 93.3 & \\
\hline $0: 18$ & $3: 33$ & 63 & 61 & 297 & 997 & 125 & 89.7 & \\
\hline $0: 20$ & $3: 35$ & 52 & 61 & 295 & 997 & 124 & 89.0 & \\
\hline $0: 22$ & $3: 37$ & 55 & 60 & 290 & 997 & 124 & 89.0 & \\
\hline $0: 24$ & $3: 39$ & 56 & 60 & 295 & 997 & 124 & 89.0 & \\
\hline $0: 26$ & $3: 41$ & 55 & 60 & 291 & 994 & 123 & 88.3 & \\
\hline $0: 28$ & $3: 43$ & 65 & 60 & 285 & 1006 & 128 & 91.9 & \\
\hline $0: 30$ & $3: 45$ & 52 & 61 & 289 & 994 & 124 & 89.0 & \\
\hline $0: 32$ & $3: 47$ & 55 & 60 & 290 & 996 & 123 & 88.3 & \\
\hline $0: 34$ & $3: 49$ & 56 & 60 & 295 & 996 & 124 & 89.0 & \\
\hline $0: 36$ & $3: 51$ & 51 & 60 & 303 & 997 & 127 & 91.2 & \\
\hline $0: 38$ & $3: 53$ & 59 & 61 & 291 & 995 & 124 & 89.0 & \\
\hline $0: 40$ & $3: 55$ & 51 & 60 & 297 & 995 & 123 & 88.3 & \\
\hline $0: 42$ & $3: 57$ & 59 & 60 & 301 & 996 & 124 & 89.0 & \\
\hline $0: 44$ & $3: 59$ & 57 & 60 & 301 & 995 & 127 & 91.2 & \\
\hline $0: 46$ & $4: 01$ & 58 & 61 & 293 & 998 & 124 & 89.0 & End Steady-state \\
\hline \multicolumn{2}{|c|}{ Steady-state Avg. } & 57 & 60 & 296 & 997 & 128 & 92 & \\
\hline \multicolumn{2}{|l|}{ Maximum } & 67 & 61 & 308 & 1011 & 137 & 98 & \\
\hline Minimum & & 50 & 59 & 280 & 993 & 123 & 88 & \\
\hline \multicolumn{2}{|c|}{ Standard Deviation } & 4.4 & 0.5 & 6.1 & 3.3 & 3.2 & 2.3 & \\
\hline \multicolumn{4}{|c|}{ TEST CONDITIONS: MATR_17.XLS } & Temp. $=$ & $60^{\circ} \mathrm{F}$ & & & \\
\hline & & & & Press. $=$ & $60 \mathrm{psig}$ & & & \\
\hline \multicolumn{3}{|c|}{ Date Run: 16,17-May-96 } & & Rotor $=$ & $1000 \mathrm{rpm}$ & & & \\
\hline & & & & Solids $=$ & $15 w \%$ & & & \\
\hline & & & & Flow $=$ & $300 \mathrm{~L} / \mathrm{hr}$ & & & \\
\hline
\end{tabular}




\section{SpinTek II High Shear Rotary Membrane System}

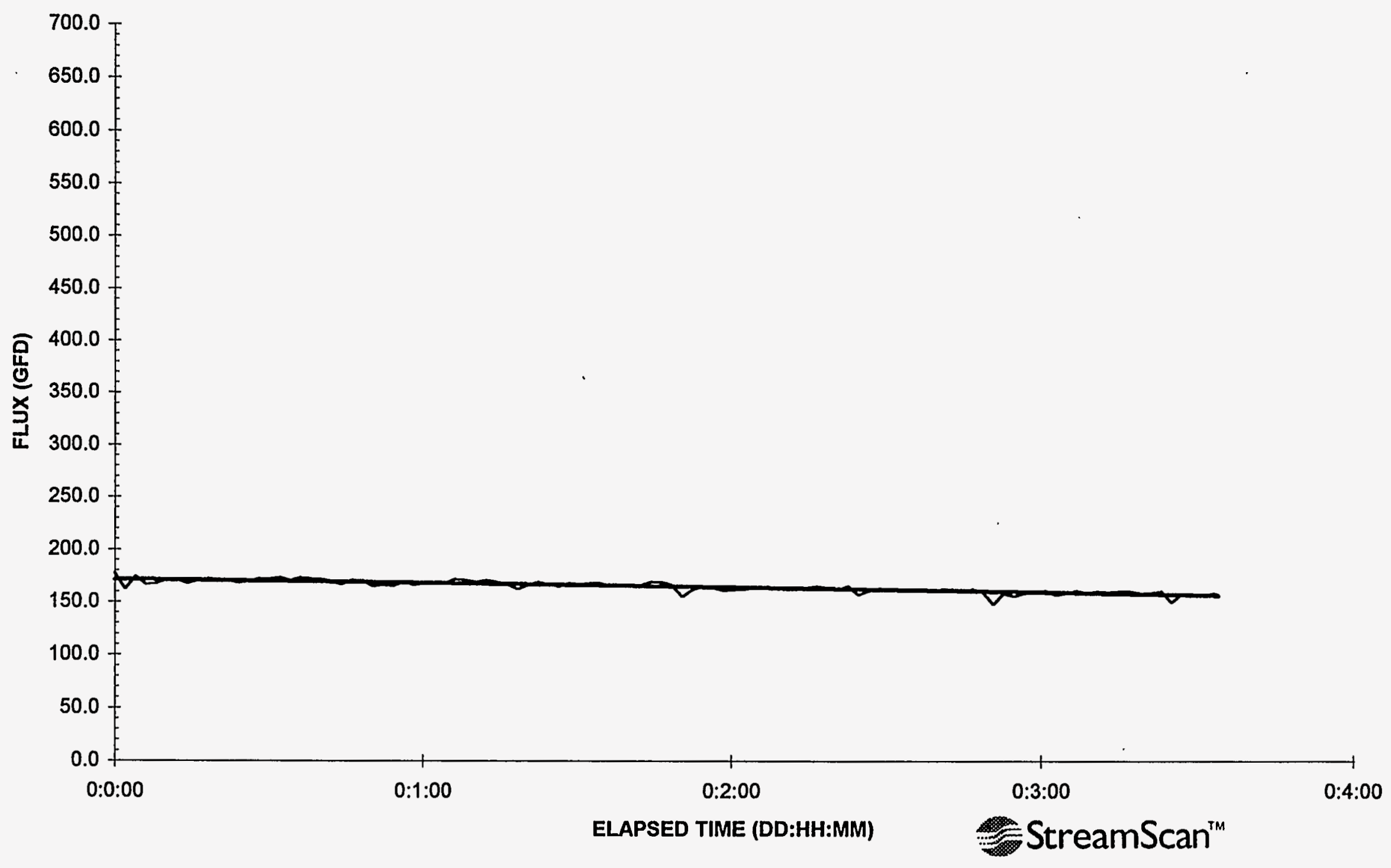




\begin{tabular}{|c|c|c|c|c|c|c|c|c|}
\hline $\begin{array}{c}\text { TIME OF } \\
\text { DAY }\end{array}$ & $\begin{array}{c}\text { ELAPSED } \\
\text { TIME }\end{array}$ & $\begin{array}{c}\text { FEED } \\
\text { PRESSURE }\end{array}$ & FEED TEMP & FEED FLOW & $\begin{array}{l}\text { ROTOR } \\
\text { SPEED }\end{array}$ & $\begin{array}{c}\text { PERMEATE } \\
\text { FLOW }\end{array}$ & \begin{tabular}{|c|} 
PERMEATE \\
FLUX
\end{tabular} & \\
\hline $3: 23$ & $0: 00$ & 84 & 90 & 275 & 997 & 247 & 177.3 & \\
\hline $3: 25$ & $0: 02$ & 79 & 90 & 272 & 995 & 226 & 162.2 & \\
\hline $3: 27$ & $0: 04$ & 74 & 88 & 265 & 993 & 242 & 173.7 & \\
\hline $3: 29$ & $0: 06$ & 78 & 88 & 291 & 993 & 232 & 166.5 & \\
\hline $3: 31$ & $0: 08$ & 83 & 88 & 288 & 995 & 233 & 167.3 & \\
\hline $3: 33$ & $0: 10$ & 74 & 88 & 296 & 996 & 238 & 170.8 & \\
\hline $3: 35$ & $0: 12$ & 70 & 88 & 293 & 997 & 238 & 170.8 & \\
\hline $3: 37$ & $0: 14$ & 82 & 88 & 285 & 996 & 233 & 167.3 & \\
\hline $3: 39$ & $0: 16$ & 80 & 88 & 292 & 995 & 237 & 170.1 & \\
\hline $3: 41$ & $0: 18$ & 75 & 88 & 284 & 996 & 240 & 172.3 & \\
\hline $3: 43$ & $0: 20$ & 73 & 89 & 282 & 993 & 237 & 170.1 & \\
\hline $3: 45$ & $0: 22$ & 81 & 89 & 284 & 994 & 237 & 170.1 & \\
\hline $3: 47$ & $0: 24$ & 81 & 89 & 289 & 995 & 234 & 168.0 & \\
\hline $3: 49$ & $0: 26$ & 81 & 89 & 284 & 997 & 236 & 169.4 & \\
\hline $3: 51$ & $0: 28$ & 82 & 89 & 289 & 994 & 240 & 172.3 & \\
\hline $3: 53$ & $0: 30$ & 77 & 89 & 280 & 993 & 239 & 171.6 & \\
\hline $3: 55$ & $0: 32$ & 80 & 89 & 288 & 996 & 241 & 173.0 & \\
\hline $3: 57$ & $0: 34$ & 74 & 89 & 283 & 993 & 237 & 170.1 & \\
\hline $3: 59$ & $0: 36$ & 73 & 89 & 286 & 1005 & 241 & 173.0 & \\
\hline $4: 01$ & $0: 38$ & 82 & 89 & 285 & 995 & 240 & 172.3 & \\
\hline $4: 03$ & $0: 40$ & 78 & 89 & 281 & 994 & 239 & 171.6 & \\
\hline $4: 05$ & $0: 42$ & 79 & 89 & 278 & 996 & 236 & 169.4 & \\
\hline $4: 07$ & $0: 44$ & 77 & 89 & 286 & 997 & 232 & 166.5 & \\
\hline $4: 09$ & $0: 46$ & 75 & 90 & 283 & 995 & 238 & 170.8 & \\
\hline $4: 11$ & $0: 48$ & 76 & 90 & 281 & 996 & 237 & 170.1 & \\
\hline $4: 13$ & $0: 50$ & 76 & 89 & 281 & 992 & 229 & 164.4 & \\
\hline $4: 15$ & $0: 52$ & 84 & 89 & 277 & 995 & 231 & 165.8 & \\
\hline $4: 17$ & $0: 54$ & 82 & 89 & 280 & 994 & 230 & 165.1 & \\
\hline $4: 19$ & $0: 56$ & 85 & 89 & 280 & 997 & 236 & 169.4 & \\
\hline $4: 21$ & $0: 58$ & 74 & 89 & 278 & 997 & 231 & 165.8 & \\
\hline $4: 23$ & $1: 00$ & 80 & 89 & 276 & 993 & 233 & 167.3 & \\
\hline $4: 25$ & $1: 02$ & 72 & 89 & 281 & 993 & 235 & 168.7 & \\
\hline $4: 27$ & $1: 04$ & 72 & 89 & 278 & 994 & 233 & 167.3 & \\
\hline $4: 29$ & $1: 06$ & 72 & 90 & 279 & 996 & 239 & 171.6 & \\
\hline $4: 31$ & $1: 08$ & 73 & 89 & 278 & 993 & 238 & 170.8 & \\
\hline $4: 33$ & $1: 10$ & 75 & 89 & 279 & 994 & 235 & 168.7 & \\
\hline $4: 35$ & $1: 12$ & 81 & 90 & 284 & 994 & 238 & 170.8 & \\
\hline $4: 37$ & $1: 14$ & 72 & 90 & 282 & 996 & 235 & 168.7 & \\
\hline $4: 39$ & $1: 16$ & 81 & 90 & 279 & 994 & 232 & 166.5 & \\
\hline $4: 41$ & $1: 18$ & 81 & 90 & 278 & 996 & 226 & 162.2 & \\
\hline $4: 43$ & $1: 20$ & 75 & 89 & 276 & 992 & 231 & 165.8 & \\
\hline $4: 45$ & $1: 22$ & 73 & 88 & 276 & 997 & 235 & 168.7 & \\
\hline $4: 47$ & $1: 24$ & 73 & 89 & 280 & 997 & 232 & 166.5 & \\
\hline $4: 49$ & $1: 26$ & 73 & 89 & 286 & 997 & 229 & 164.4 & \\
\hline $4: 51$ & $1: 28$ & 80 & 89 & 272 & 994 & 234 & 168.0 & \\
\hline
\end{tabular}




\begin{tabular}{|c|c|c|c|c|c|c|c|c|}
\hline $\begin{array}{c}\text { TIME OF } \\
\text { DAY }\end{array}$ & $\begin{array}{c}\text { ELAPSED } \\
\text { TIME }\end{array}$ & $\begin{array}{c}\text { FEED } \\
\text { PRESSURE }\end{array}$ & FEED TEMP & FEED FLOW & $\begin{array}{l}\text { ROTOR } \\
\text { SPEED } \\
\end{array}$ & $\begin{array}{c}\text { PERMEATE } \\
\text { FLOW }\end{array}$ & $\begin{array}{c}\text { PERMEATE } \\
\text { FLUX } \\
\end{array}$ & \\
\hline $4: 53$ & $1: 30$ & 83 & 89 & 279 & 1000 & 232 & 166.5 & \\
\hline $4: 55$ & $1: 32$ & $\overline{81}$ & 89 & 275 & 999 & 234 & 168.0 & \\
\hline $4: 57$ & $1: 34$ & 77 & 89 & 279 & 992 & 234 & 168.0 & \\
\hline $4: 59$ & $1: 36$ & 85 & 89 & 278 & 998 & 229 & 164.4 & \\
\hline 5:01 & $1: 38$ & 73 & 89 & 280 & 993 & 231 & 165.8 & \\
\hline $5: 03$ & $1: 40$ & 75 & 90 & 279 & 997 & 231 & 165.8 & \\
\hline $5: 05$ & $1: 42$ & 76 & 89 & 271 & 996 & 231 & 165.8 & \\
\hline $5: 07$ & $1: 44$ & 78 & 90 & 280 & 993 & 236 & 169.4 & \\
\hline $5: 09$ & $1: 46$ & 74 & 90 & 276 & 997 & 235 & 168.7 & \\
\hline $5: 11$ & $1: 48$ & 81 & 90 & 275 & 995 & 231 & 165.8 & \\
\hline $5: 13$ & $1: 50$ & 73 & 89 & 274 & 992 & 216 & 155.1 & Begin Steady-state \\
\hline $5: 15$ & $1: 52$ & 85 & 89 & 273 & 999 & 225 & 161.5 & \\
\hline $5: 18$ & $1: 54$ & 81 & 88 & 275 & 996 & 229 & 164.4 & \\
\hline $5: 20$ & $1: 56$ & 85 & 88 & 278 & 994 & 228 & 163.7 & \\
\hline $5: 22$ & $1: 58$ & 72 & 89 & 276 & 994 & 224 & 160.8 & \\
\hline $5: 24$ & $2: 00$ & 82 & 88 & 278 & 994 & 225 & 161.5 & \\
\hline $5: 26$ & $2: 02$ & 74 & 89 & 277 & 999 & 226 & 162.2 & \\
\hline $5: 28$ & 2:04 & 80 & 89 & 272 & 994 & 227 & 162.9 & \\
\hline $5: 30$ & $2: 06$ & 85 & 89 & 273 & 994 & 229 & 164.4 & \\
\hline $5: 32$ & $2: 08$ & 79 & 89 & 275 & $\overline{996}$ & 227 & 162.9 & \\
\hline $5: 34$ & $2: 10$ & 78 & 89 & 275 & 995 & 225 & 161.5 & \\
\hline $5: 36$ & $2: 12$ & 81 & 89 & 274 & 996 & 226 & 162.2 & \\
\hline $5: 38$ & $2: 14$ & 82 & 89 & 280 & 996 & 228 & 163.7 & \\
\hline $5: 40$ & $2: 16$ & 82 & 90 & 274 & 997 & 230 & 165.1 & \\
\hline $5: 42$ & $2: 18$ & 77 & 89 & 275 & 993 & 227 & 162.9 & \\
\hline $5: 44$ & $2: 20$ & 83 & 90 & 277 & 996 & 225 & 161.5 & \\
\hline $5: 46$ & $2: 22$ & 80 & 90 & 277 & 995 & 230 & 165.1 & \\
\hline $5: 48$ & $2: 24$ & 73 & 89 & 276 & 996 & 219 & 157.2 & \\
\hline $5: 50$ & $2: 26$ & 83 & 89 & 277 & 997 & 224 & 160.8 & \\
\hline $5: 52$ & $2: 28$ & 78 & 89 & 272 & 997 & 227 & 162.9 & \\
\hline $5: 54$ & $2: 30$ & 83 & 89 & 278 & 993 & 226 & 162.2 & \\
\hline $5: 56$ & $2: 32$ & 82 & 89 & 278 & 993 & 226 & 162.2 & \\
\hline $5: 58$ & $2: 34$ & 73 & 89 & 276 & 994 & 226 & 162.2 & \\
\hline $6: 00$ & $2: 36$ & 74 & 89 & 274 & 997 & 226 & 162.2 & \\
\hline $6: 02$ & $2: 38$ & 77 & 89 & 276 & 993 & 225 & 161.5 & \\
\hline $6: 04$ & $2: 40$ & 76 & 89 & 275 & 996 & 226 & 162.2 & \\
\hline $6: 06$ & $2: 43$ & 82 & 90 & 274 & 997 & 226 & 162.2 & \\
\hline $6: 08$ & $2: 45$ & 75 & 90 & 274 & 995 & 225 & 161.5 & \\
\hline $6: 10$ & $2: 47$ & 75 & 90 & 274 & 994 & 226 & 162.2 & \\
\hline $6: 12$ & $2: 49$ & $\overline{72}$ & 90 & 275 & 997 & 222 & 159.4 & \\
\hline $6: 14$ & $2: 51$ & 79 & 90 & 269 & 993 & 206 & 147.9 & \\
\hline $6: 16$ & $2: 53$ & 73 & 88 & 279 & 994 & 220 & 157.9 & \\
\hline $6: 18$ & $2: 55$ & . 83 & 88 & 278 & 995 & 217 & 155.8 & \\
\hline $6: 20$ & $2: 57$ & 73 & 88 & 274 & 995 & 221 & 158.6 & \\
\hline $6: 22$ & $2: 59$ & 84 & 89 & 273 & 997 & 221 & 158.6 & \\
\hline $6: 24$ & $3: 01$ & 71 & 89 & 271 & 997 & 224 & 160.8 & \\
\hline $6: 26$ & $3: 03$ & 80 & 89 & 278 & 997 & 218 & 156.5 & \\
\hline $6: 28$ & $3: 05$ & 73 & 89 & 277 & 995 & 221 & 158.6 & \\
\hline $6: 30$ & $3: 07$ & 75 & 89 & 270 & 996 & 224 & 160.8 & \\
\hline $6: 32$ & $3: 09$ & 76 & 89 & 272 & 998 & 221 & 158.6 & \\
\hline
\end{tabular}

Page 2 
MATR_18.XLS

\begin{tabular}{|c|c|c|c|c|c|c|c|c|}
\hline $\begin{array}{c}\text { TIME OF } \\
\text { DAY }\end{array}$ & $\begin{array}{c}\text { ELAPSED } \\
\text { TIME }\end{array}$ & $\begin{array}{c}\text { FEED } \\
\text { PRESSURE }\end{array}$ & FEED TEMP & FEED FLOW & $\begin{array}{l}\text { ROTOR } \\
\text { SPEED }\end{array}$ & $\begin{array}{c}\text { PERMEATE } \\
\text { FLOW }\end{array}$ & \begin{tabular}{|c|} 
PERMEATE \\
FLUX
\end{tabular} & \\
\hline $6: 34$ & $3: 11$ & 78 & 90 & 278 & 997 & 223 & 160.1 & \\
\hline $6: 36$ & $3: 13$ & 75 & 90 & 276 & 995 & 222 & 159.4 & \\
\hline $6: 38$ & $3: 15$ & 79 & 90 & 274 & 996 & 223 & 160.1 & \\
\hline $6: 40$ & $3: 17$ & 74 & 90 & 278 & 994 & 223 & 160.1 & \\
\hline $6: 42$ & $3: 19$ & 72 & 90 & 276 & 997 & 221 & 158.6 & \\
\hline $6: 44$ & $3: 21$ & 77 & 90 & 278 & 994 & 221 & 158.6 & \\
\hline $6: 46$ & $3: 23$ & 72 & 90 & 279 & 993 & 223 & 160.1 & \\
\hline $6: 48$ & $3: 25$ & 78 & 89 & 271 & 997 & 209 & 150.0 & \\
\hline $6: 50$ & $3: 27$ & 85 & 88 & 276 & 995 & 220 & 157.9 & \\
\hline $6: 52$ & $3: 29$ & 75 & 88 & 275 & 994 & 220 & 157.9 & \\
\hline $6: 54$ & $3: 31$ & 71 & 89 & 273 & 992 & 217 & 155.8 & \\
\hline $6: 56$ & $3: 33$ & 81 & 89 & 272 & 997 & 221 & 158.6 & \\
\hline $6: 57$ & $3: 34$ & 78 & 89 & 275 & 994 & 219 & 157.2 & End Steady-state \\
\hline \multicolumn{2}{|c|}{ Steady-state Avg. } & 78 & 89 & 275 & 995 & 223 & 160 & \\
\hline \multicolumn{2}{|l|}{ Maximum } & 85 & 90 & 280 & 999 & 230 & 165 & \\
\hline Minimum & & 71 & 88 & 269 & 992 & 206 & 148 & \\
\hline \multicolumn{2}{|c|}{ Standard Deviation } & 4.3 & 0.7 & 2.5 & 1.7 & 4.6 & 3.3 & \\
\hline \multicolumn{4}{|c|}{ TEST CONDITIONS: MATR_18.XLS } & Temp. $=$ & $90^{\circ} \mathrm{F}$ & & & \\
\hline & & & & Press. $=$ & 75 psig & & & \\
\hline \multicolumn{3}{|c|}{ Date Run: 17-May-96 } & & Rotor $=$ & $1000 \mathrm{rpm}$ & & & \\
\hline & & & & Solids = & $15 w t \%$ & & & \\
\hline & & & & Flow $=$ & $300 \mathrm{~L} / \mathrm{hr}$ & & & \\
\hline
\end{tabular}




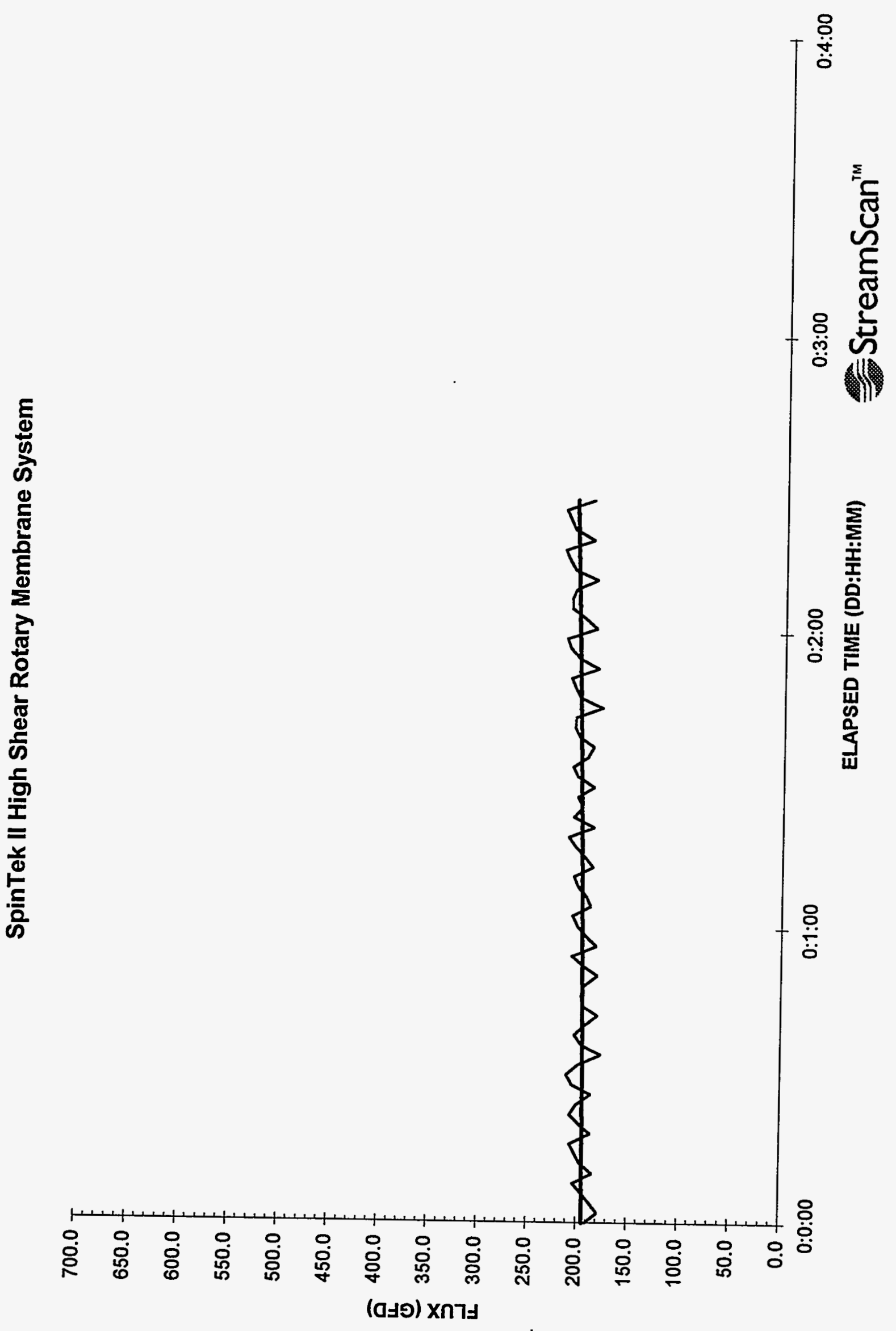




\begin{tabular}{|c|c|c|c|c|c|c|c|c|}
\hline $\begin{array}{c}\text { TIME OF } \\
\text { DAY }\end{array}$ & $\begin{array}{l}\text { ELAPSED } \\
\text { TIME }\end{array}$ & $\begin{array}{c}\text { FEED } \\
\text { PRESSURE }\end{array}$ & FEED TEMP & FEED FLOW & $\begin{array}{l}\text { ROTOR } \\
\text { SPEED }\end{array}$ & $\begin{array}{c}\text { PERMEATE } \\
\text { FLOW }\end{array}$ & \begin{tabular}{|c|} 
PERMEATE \\
FLUX
\end{tabular} & \\
\hline $7: 36$ & $0: 00$ & 60 & 90 & 289 & 1506 & 265 & 190.2 & \\
\hline $7: 38$ & $0: 02$ & 63 & 90 & 287 & 1507 & 249 & 178.7 & \\
\hline $7: 40$ & $0: 04$ & 59 & 88 & 291 & 1502 & 260 & 186.6 & \\
\hline $7: 42$ & $0: 06$ & 62 & 89 & 287 & 1497 & 271 & 194.5 & \\
\hline $7: 44$ & $0: 08$ & 64 & 90 & 290 & 1503 & 283 & 203.1 & \\
\hline $7: 46$ & $0: 10$ & 63 & 91 & 295 & 1493 & 257 & 184.5 & \\
\hline $7: 48$ & $0: 12$ & 66 & 89 & 295 & 1503 & 274 & 196.7 & \\
\hline $7: 50$ & $0: 14$ & 58 & 89 & 292 & 1503 & 281 & 201.7 & \\
\hline $7: 52$ & $0: 16$ & 59 & 90 & 297 & 1491 & 288 & 206.7 & \\
\hline $7: 54$ & $0: 18$ & 71 & 90 & 288 & 1491 & 260 & 186.6 & \\
\hline $7: 56$ & $0: 20$ & 66 & 89 & 293 & 1494 & 275 & 197.4 & \\
\hline $7: 58$ & $0: 22$ & 57 & 89 & 291 & 1501 & 289 & 207.5 & \\
\hline $8: 00$ & $0: 24$ & 66 & 90 & 293 & 1499 & 280 & 201.0 & Begin Steady-state \\
\hline $8: 02$ & $0: 26$ & 67 & 90 & 293 & 1497 & 260 & 186.6 & \\
\hline $8: 04$ & $0: 28$ & 74 & 89 & 285 & 1505 & 286 & 205.3 & \\
\hline 8:06 & $0: 30$ & 56 & 90 & 288 & 1503 & 294 & 211.0 & \\
\hline $8: 08$ & $0: 32$ & 68 & 90 & 291 & 1507 & 278 & 199.6 & \\
\hline $8: 10$ & $0: 34$ & 60 & 90 & 302 & 1504 & 247 & 177.3 & \\
\hline $8: 12$ & $0: 36$ & 60 & 89 & 304 & 1499 & 274 & 196.7 & \\
\hline $8: 14$ & $0: 38$ & 54 & 90 & 306 & 1503 & 284 & 203.9 & \\
\hline $8: 16$ & $0: 40$ & 61 & 91 & 306 & 1506 & 270 & 193.8 & \\
\hline $8: 18$ & $0: 42$ & 53 & 90 & 306 & 1505 & 252 & 180.9 & \\
\hline $8: 20$ & $0: 44$ & 60 & 89 & 305 & 1506 & 273 & 196.0 & \\
\hline $8: 22$ & $0: 46$ & 53 & 90 & 306 & 1494 & 275 & 197.4 & \\
\hline $8: 24$ & $0: 48$ & 54 & 90 & 309 & 1502 & 271 & 194.5 & \\
\hline $8: 26$ & $0: 50$ & 60 & 89 & 305 & 1491 & 253 & 181.6 & \\
\hline $8: 28$ & $0: 52$ & 63 & 89 & 310 & 1490 & 272 & 195.2 & \\
\hline $8: 30$ & $0: 54$ & 61 & 90 & 316 & 1489 & 288 & 206.7 & \\
\hline $8: 32$ & $0: 56$ & 55 & 91 & 304 & 1491 & 255 & 183.0 & \\
\hline $8: 34$ & $0: 58$ & 63 & 89 & 304 & 1501 & 269 & 193.1 & \\
\hline $8: 36$ & $1: 00$ & 52 & 89 & 312 & 1505 & 281 & 201.7 & \\
\hline $8: 38$ & $1: 02$ & 54 & 90 & 301 & 1497 & 288 & 206.7 & \\
\hline $8: 40$ & $1: 04$ & 53 & 91 & 309 & 1503 & 263 & 188.8 & \\
\hline $8: 42$ & $1: 06$ & 60 & 89 & 310 & 1503 & 269 & 193.1 & \\
\hline $8: 44$ & $1: 08$ & 52 & 89 & 308 & 1497 & 281 & 201.7 & \\
\hline $8: 46$ & $1: 10$ & 63 & 90 & 307 & 1503 & 287 & 206.0 & \\
\hline $8: 48$ & $1: 12$ & 61 & 90 & 303 & 1497 & 261 & 187.4 & \\
\hline $8: 50$ & $1: 14$ & 58 & 89 & 305 & 1504 & 272 & 195.2 & \\
\hline $8: 52$ & $1: 16$ & 56 & 89 & 299 & 1499 & 285 & 204.6 & \\
\hline $8: 55$ & $1: 18$ & 69 & 90 & 309 & 1496 & 295 & 211.8 & \\
\hline $8: 57$ & $1: 20$ & 63 & 90 & 305 & 1498 & 260 & 186.6 & \\
\hline $8: 59$ & $1: 22$ & 55 & 89 & 308 & 1502 & 288 & 206.7 & \\
\hline $9: 01$ & $1: 24$ & 69 & 89 & 306 & 1492 & 276 & 198.1 & \\
\hline $9: 03$ & $1: 26$ & 53 & 90 & 309 & 1496 & 283 & 203.1 & \\
\hline $9: 05$ & $1: 28$ & 62 & 90 & 308 & 1489 & 261 & 187.4 & \\
\hline
\end{tabular}


MATR_19.XLS

\begin{tabular}{|c|c|c|c|c|c|c|c|c|}
\hline $\begin{array}{c}\text { TIME OF } \\
\text { DAY }\end{array}$ & $\begin{array}{c}\text { ELAPSED } \\
\text { TIME }\end{array}$ & \begin{tabular}{c|} 
FEED \\
PRESSURE \\
\end{tabular} & FEED TEMP & FEED FLOW & $\begin{array}{l}\text { ROTOR } \\
\text { SPEED }\end{array}$ & $\begin{array}{c}\text { PERMEATE } \\
\text { FLOW }\end{array}$ & $\begin{array}{c}\text { PERMEATE } \\
\text { FLUX }\end{array}$ & \\
\hline $9: 07$ & $1: 30$ & 63 & 89 & 300 & 1505 & 283 & 203.1 & \\
\hline $9: 09$ & $1: 32$ & 52 & 89 & 315 & 1503 & 290 & 208.2 & \\
\hline $9: 11$ & $1: 34$ & 52 & 91 & 308 & 1491 & 270 & 193.8 & \\
\hline $9: 13$ & $1: 36$ & 54 & 89 & 302 & 1506 & 262 & 188.1 & \\
\hline $9: 15$ & $1: 38$ & 57 & 89 & 308 & 1496 & 282 & 202.4 & \\
\hline $9: 17$ & $1: 40$ & 54 & 90 & 308 & 1500 & 288 & 206.7 & \\
\hline $9: 19$ & $1: 42$ & 64 & 90 & 313 & 1500 & 287 & 206.0 & \\
\hline $9: 21$ & $1: 44$ & 63 & 90 & 307 & 1503 & 250 & 179.5 & \\
\hline $9: 23$ & $1: 46$ & 54 & 88 & 308 & 1506 & 281 & 201.7 & \\
\hline $9: 25$ & $1: 48$ & 66 & 90 & 312 & 1495 & 288 & 206.7 & \\
\hline $9: 27$ & $1: 50$ & 69 & 90 & 311 & 1501 & 294 & 211.0 & \\
\hline $9: 29$ & $1: 52$ & 63 & 90 & 306 & 1503 & 257 & 184.5 & \\
\hline $9: 31$ & $1: 54$ & 62 & 88 & 307 & 1502 & 283 & 203.1 & \\
\hline $9: 33$ & $1: 56$ & 57 & 89 & 301 & 1501 & 296 & 212.5 & \\
\hline $9: 35$ & $1: 58$ & 59 & 90 & 314 & 1505 & 301 & 216.1 & \\
\hline $9: 37$ & $2: 00$ & 55 & 91 & 309 & 1490 & 260 & 186.6 & \\
\hline $9: 39$ & $2: 02$ & 65 & 89 & 303 & 1493 & 276 & 198.1 & \\
\hline $9: 41$ & $2: 04$ & 54 & 89 & 308 & 1501 & 294 & 211.0 & \\
\hline $9: 43$ & $2: 06$ & 52 & 90 & 304 & 1500 & 295 & 211.8 & \\
\hline $9: 45$ & $2: 08$ & 53 & 90 & 306 & 1502 & 289 & 207.5 & \\
\hline $9: 47$ & $2: 10$ & 69 & 90 & 307 & 1495 & 260 & 186.6 & \\
\hline $9: 49$ & $2: 12$ & 53 & 88 & 303 & 1503 & 290 & 208.2 & \\
\hline $9: 51$ & $2: 14$ & 62 & 89 & 304 & 1503 & 299 & 214.6 & \\
\hline $9: 53$ & $2: 16$ & 52 & 90 & 300 & 1496 & 305 & 218.9 & \\
\hline $9: 55$ & $2: 18$ & 58 & 90 & 303 & 1499 & 265 & 190.2 & \\
\hline $9: 57$ & $2: 20$ & 53 & 89 & 304 & 1489 & 291 & 208.9 & \\
\hline $9: 59$ & $2: 22$ & 68 & 89 & 308 & 1490 & 298 & 213.9 & \\
\hline $10: 01$ & $2: 24$ & 55 & 90 & 307 & 1502 & 304 & 218.2 & \\
\hline $10: 03$ & $2: 26$ & 52 & 91 & 316 & 1503 & 265 & 190.2 & End Steady-state \\
\hline & & & & & & & & \\
\hline \multicolumn{2}{|c|}{ Steady-state Avg. } & 59 & 90 & 305 & 1499 & 278 & 200 & \\
\hline \multicolumn{2}{|l|}{ Maximum } & 74 & 91 & 316 & 1507 & 305 & 219 & \\
\hline Minimum & & 52 & 88 & 285 & 1489 & 247 & 177 & \\
\hline \multicolumn{2}{|c|}{ Standard Deviation } & 5.8 & 0.7 & 6.0 & 5.2 & 14.7 & 10.5 & \\
\hline \multicolumn{4}{|c|}{ TEST CONDITIONS: MATR_19.XLS } & Temp. $=$ & $90^{\circ} \mathrm{F}$ & & & \\
\hline & & & & Press. $=$ & 60 psig & & & \\
\hline \multicolumn{3}{|c|}{ Date Run: 17-May-96 } & & Rotor $=$ & $1500 \mathrm{rpm}$ & & & \\
\hline & & & & Solids $=$ & $15 w \%$ & & & \\
\hline & & & & Flow $=$ & $300 \mathrm{~L} / \mathrm{hr}$ & & & \\
\hline
\end{tabular}




\section{SpinTek II High Shear Rotary Membrane System}

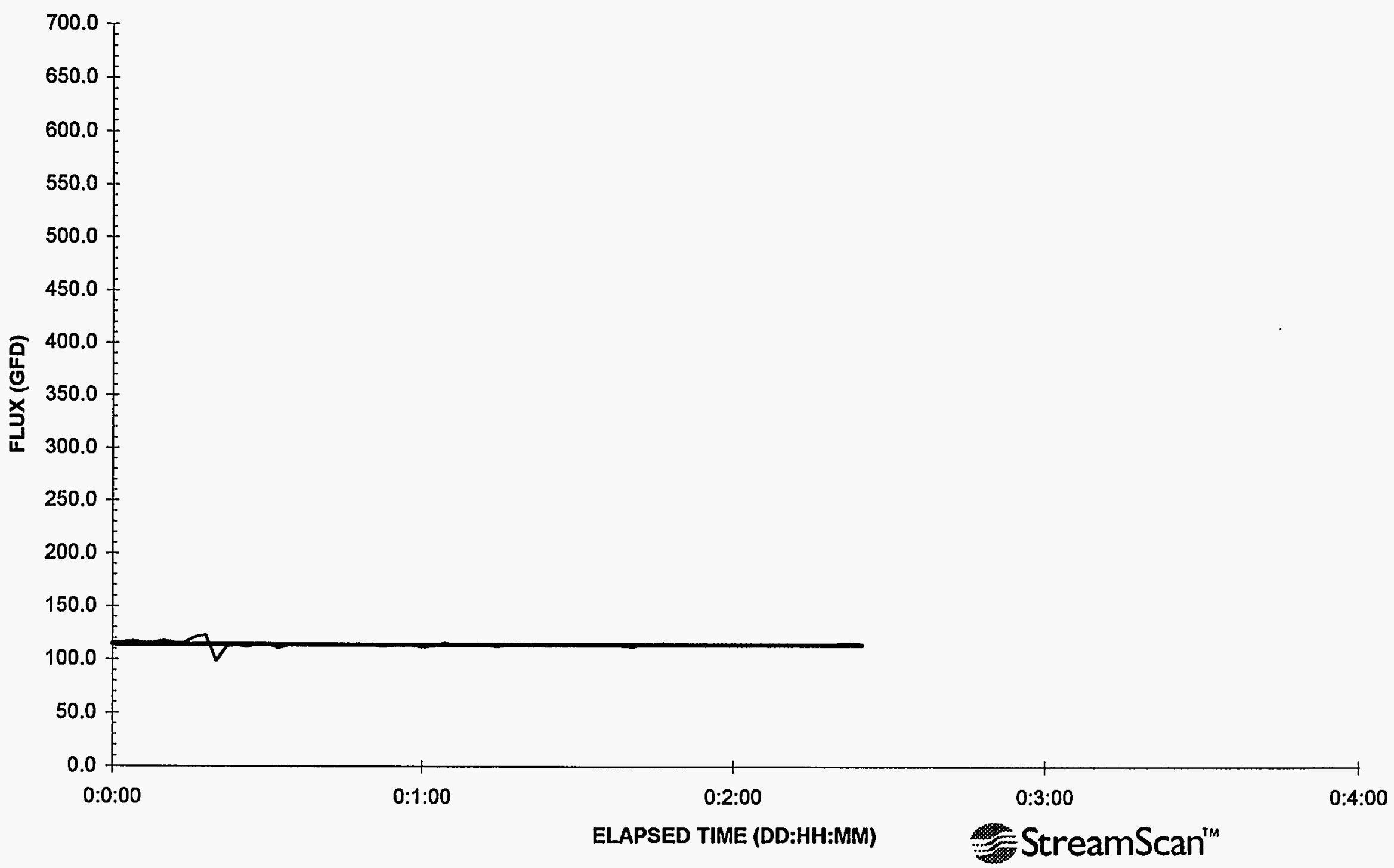




\begin{tabular}{|c|c|c|c|c|c|c|c|c|}
\hline $\begin{array}{c}\text { TIME OF } \\
\text { DAY }\end{array}$ & $\begin{array}{l}\text { ELAPSED } \\
\text { TIME }\end{array}$ & $\begin{array}{c}\text { FEED } \\
\text { PRESSURE }\end{array}$ & FEED TEMP & FEED FLOW & $\begin{array}{l}\text { ROTOR } \\
\text { SPEED }\end{array}$ & $\begin{array}{l}\text { PERMEATE } \\
\text { FLOW }\end{array}$ & $\begin{array}{c}\text { PERMEATE } \\
\text { FLUX }\end{array}$ & \\
\hline $11: 15$ & $0: 00$ & 45 & 61 & 311 & 1501 & 162 & 116.3 & \\
\hline $11: 17$ & $0: 02$ & 42 & 61 & 311 & 1503 & 162 & 116.3 & \\
\hline $11: 19$ & $0: 04$ & 44 & 61 & 298 & $1504^{\circ}$ & 163 & 117.0 & \\
\hline $11: 21$ & $0: 06$ & 42 & 60 & 313 & 1496 & 161 & 115.6 & \\
\hline $11: 23$ & $0: 08$ & 49 & 61 & 306 & 1500 & 161 & 115.6 & \\
\hline $11: 25$ & $0: 10$ & 41 & 61 & 307 & 1500 & 164 & 117.7 & \\
\hline $11: 27$ & $0: 12$ & 42 & 61 & 306 & 1505 & 161 & 115.6 & \\
\hline $11: 29$ & $0: 14$ & 42 & 60 & 317 & 1492 & 161 & 115.6 & \\
\hline $11: 31$ & $0: 16$ & 43 & 60 & 294 & 1503 & 168 & 120.6 & \\
\hline $11: 33$ & $0: 18$ & 50 & 60 & 284 & 1502 & 171 & 122.7 & \\
\hline $11: 35$ & $0: 20$ & 39 & 60 & 318 & 1498 & 137 & 98.3 & \\
\hline $11: 37$ & $0: 22$ & 43 & 60 & 306 & 1502 & 156 & 112.0 & Begin Steady-state \\
\hline $11: 39$ & $0: 24$ & 48 & 61 & 315 & 1503 & 158 & 113.4 & \\
\hline $11: 41$ & $0: 26$ & 40 & 60 & 308 & 1496 & 155 & 111.3 & \\
\hline $11: 43$ & $0: 28$ & 43 & 60 & 310 & 1503 & 159 & 114.1 & \\
\hline $11: 45$ & $0: 30$ & 44 & 60 & 314 & 1509 & 159 & 114.1 & \\
\hline $11: 47$ & $0: 32$ & 44 & 60 & 315 & 1501 & 154 & 110.5 & \\
\hline $11: 49$ & $0: 34$ & 40 & 60 & 309 & 1504 & 158 & 113.4 & \\
\hline $11: 51$ & $0: 36$ & 43 & 60 & 295 & 1499 & 160 & 114.9 & \\
\hline $11: 53$ & $0: 38$ & 43 & 61 & 308 & 1504 & 157 & 112.7 & \\
\hline $11: 55$ & $0: 40$ & 39 & 60 & 314 & 1497 & 157 & 112.7 & \\
\hline $11: 57$ & $0: 42$ & 44 & 60 & 308 & 1497 & 159 & 114.1 & \\
\hline $11: 59$ & $0: 44$ & 41 & 60 & 313 & 1511 & 157 & 112.7 & \\
\hline $12: 01$ & $0: 46$ & 47 & 60 & 306 & 1503 & 157 & 112.7 & \\
\hline $12: 03$ & $0: 48$ & 40 & 61 & 306 & 1503 & 159 & 114.1 & \\
\hline $12: 05$ & $0: 50$ & 39 & 60 & 312 & 1501 & 158 & 113.4 & \\
\hline $12: 07$ & $0: 52$ & 40 & 60 & 303 & 1499 & 156 & 112.0 & \\
\hline $12: 09$ & $0: 54$ & 48 & 60 & 307 & 1501 & 157 & 112.7 & \\
\hline $12: 11$ & $0: 56$ & 43 & 60 & 305 & 1504 & 159 & 114.1 & \\
\hline $12: 13$ & $0: 58$ & 47 & 60 & 313 & 1503 & 157 & 112.7 & \\
\hline 12:15 & $1: 00$ & 44 & 60 & 303 & 1504 & 155 & 111.3 & \\
\hline $12: 17$ & $1: 02$ & 39 & 60 & 301 & 1503 & 157 & 112.7 & \\
\hline $12: 19$ & $1: 04$ & 44 & 60 & 314 & 1502 & 161 & 115.6 & \\
\hline $12: 21$ & $1: 06$ & 42 & 60 & 308 & 1491 & 158 & 113.4 & \\
\hline $12: 23$ & $1: 08$ & 39 & 60 & 303 & 1503 & 160 & 114.9 & \\
\hline $12: 25$ & $1: 10$ & 48 & 60 & 301 & 1499 & 158 & 113.4 & \\
\hline $12: 27$ & $1: 12$ & 46 & 60 & 314 & 1504 & 158 & 113.4 & \\
\hline $12: 29$ & $1: 14$ & 40 & 60 & 321 & 1510 & 156 & 112.0 & \\
\hline $12: 31$ & $1: 16$ & 46 & 61 & 318 & 1508 & 158 & 113.4 & \\
\hline $12: 33$ & $1: 18$ & 42 & 60 & 308 & 1501 & 158 & 113.4 & \\
\hline $12: 35$ & $1: 20$ & 43 & 60 & 299 & 1499 & 158 & 113.4 & \\
\hline $12: 37$ & $1: 22$ & 42 & 60 & 311 & 1498 & 158 & 113.4 & \\
\hline $12: 39$ & $1: 24$ & 42 & 60 & 290 & 1503 & 159 & 114.1 & \\
\hline $12: 41$ & $1: 26$ & 41 & 61 & 307 & 1495 & 158 & 113.4 & \\
\hline $12: 43$ & $1: 28$ & 47 & 61 & 307 & 1499 & 159 & 114.1 & \\
\hline
\end{tabular}


MATR_20.XLS

\begin{tabular}{|c|c|c|c|c|c|c|c|c|}
\hline $\begin{array}{c}\text { TIME OF } \\
\text { DAY }\end{array}$ & $\begin{array}{c}\text { ELAPSED } \\
\text { TIME }\end{array}$ & $\begin{array}{c}\text { FEED } \\
\text { PRESSURE }\end{array}$ & FEED TEMP & FEED FLOW & $\begin{array}{l}\text { ROTOR } \\
\text { SPEED }\end{array}$ & $\begin{array}{c}\text { PERMEATE } \\
\text { FLOW }\end{array}$ & \begin{tabular}{|c|} 
PERMEATE \\
FLUX
\end{tabular} & \\
\hline $12: 45$ & $1: 30$ & 40 & 61 & 319 & 1502 & 158 & 113.4 & \\
\hline $12: 47$ & $1: 32$ & 40 & 60 & 300 & 1503 & 158 & 113.4 & \\
\hline $12: 49$ & $1: 34$ & 40 & 60 & 301 & 1501 & 159 & 114.1 & \\
\hline $12: 51$ & $1: 36$ & 44 & 60 & 312 & 1496 & 158 & 113.4 & \\
\hline $12: 53$ & $1: 38$ & 39 & 60 & 312 & 1495 & 157 & 112.7 & \\
\hline $12: 55$ & $1: 40$ & 44 & 60 & 294 & 1503 & 156 & 112.0 & \\
\hline $12: 57$ & $1: 42$ & 47 & 60 & 318 & 1496 & 159 & 114.1 & \\
\hline $12: 59$ & $1: 44$ & 44 & 61 & 305 & 1489 & 160 & 114.9 & \\
\hline $13: 01$ & $1: 46$ & 39 & 61 & 298 & 1503 & 161 & 115.6 & \\
\hline $13: 03$ & $1: 48$ & 39 & 60 & 313 & 1503 & 160 & 114.9 & \\
\hline $13: 05$ & $1: 50$ & 41 & 60 & 304 & 1502 & 160 & 114.9 & \\
\hline $13: 08$ & $1: 52$ & 43 & 60 & 305 & 1498 & 158 & 113.4 & \\
\hline $13: 10$ & $1: 54$ & 41 & 60 & 307 & 1500 & 160 & 114.9 & \\
\hline $13: 12$ & $1: 56$ & 42 & 60 & 315 & 1501 & 160 & 114.9 & \\
\hline $13: 14$ & $1: 58$ & 43 & 60 & 307 & 1498 & 158 & 113.4 & \\
\hline $13: 16$ & $2: 00$ & 41 & 60 & 310 & 1500 & 159 & 114.1 & \\
\hline $13: 18$ & $2: 02$ & 44 & 60 & 307 & 1501 & 159 & 114.1 & \\
\hline $13: 20$ & $2: 04$ & 43 & 60 & 295 & 1500 & 160 & 114.9 & \\
\hline $13: 22$ & $2: 06$ & 44 & 60 & 303 & 1501 & 158 & 113.4 & \\
\hline $13: 24$ & $2: 08$ & 40 & 60 & 309 & 1499 & 158 & 113.4 & \\
\hline 13:26 & $2: 10$ & 41 & 60 & 296 & 1508 & 159 & 114.1 & \\
\hline $13: 28$ & $2: 12$ & 44 & 61 & 300 & 1500 & 160 & 114.9 & \\
\hline $13: 30$ & $2: 14$ & 39 & 61 & 303 & 1490 & 160 & 114.9 & \\
\hline $13: 32$ & $2: 16$ & 41 & 61 & 305 & 1496 & 160 & 114.9 & \\
\hline $13: 34$ & $2: 18$ & 43 & 60 & 296 & 1497 & 157 & 112.7 & \\
\hline $13: 36$ & $2: 20$ & 44 & 60 & 306 & 1499 & 161 & 115.6 & \\
\hline $13: 38$ & $2: 22$ & 48 & 61 & 317 & 1506 & 161 & 115.6 & \\
\hline $13: 40$ & $2: 24$ & 41 & 61 & 310 & 1491 & 160 & 114.9 & End Steady-state \\
\hline \multicolumn{2}{|c|}{ Steady-state Avg. } & 43 & 60 & 307 & 1501 & 158 & 114 & \\
\hline \multicolumn{2}{|c|}{ Maximum } & 48 & 61 & 321 & 1511 & 161 & 116 & \\
\hline Minimum & & 39 & 60 & 290 & 1489 & 154 & 111 & \\
\hline \multicolumn{2}{|c|}{ Standard Deviation } & 2.7 & 0.4 & 6.7 & 4.4 & 1.6 & 1.1 & \\
\hline \multicolumn{4}{|c|}{ TEST CONDITIONS: MATR_20.XLS } & Temp. $=$ & $60 \mathrm{~F}$ & & & \\
\hline & & & & Press. = & 45 psig & & & \\
\hline \multicolumn{3}{|c|}{ Date Run: 17-May-96 } & & Rotor $=$ & $1500 \mathrm{rpm}$ & & & \\
\hline & & & & Solids $=$ & $15 w t \%$ & & & \\
\hline & & & & Flow $=$ & $400 \mathrm{~L} / \mathrm{hr}$ & & & \\
\hline
\end{tabular}




\section{SpinTek II High Shear Rotary Membrane System}

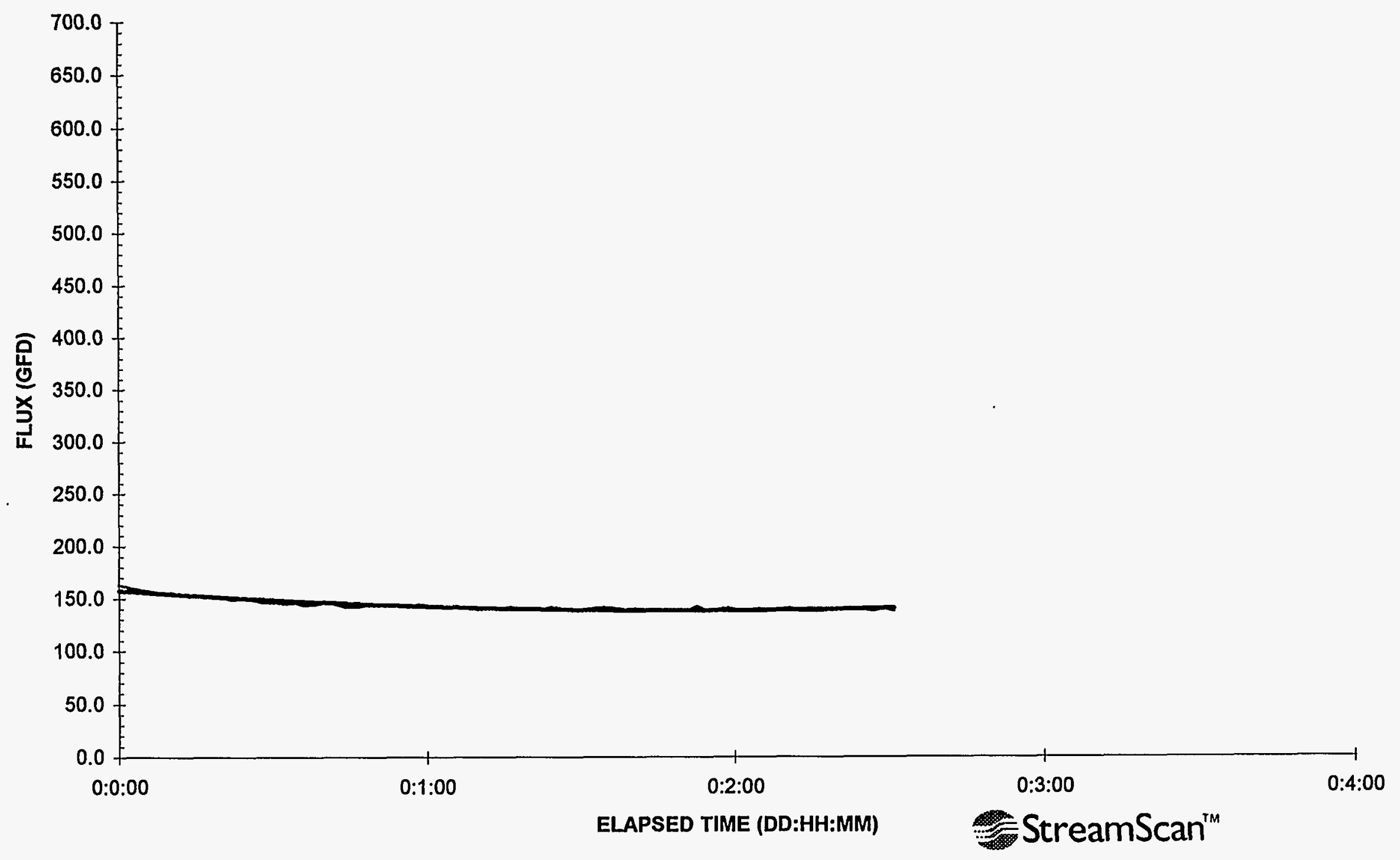




\begin{tabular}{|c|c|c|c|c|c|c|c|c|}
\hline $\begin{array}{c}\text { TIME OF } \\
\text { DAY }\end{array}$ & $\begin{array}{c}\text { ELAPSED } \\
\text { TIME }\end{array}$ & \begin{tabular}{|c|c} 
FEED \\
PRESSURE
\end{tabular} & FEED TEMP & FEED FLOW & $\begin{array}{l}\text { ROTOR } \\
\text { SPEED }\end{array}$ & $\begin{array}{c}\text { PERMEATE } \\
\text { FLOW }\end{array}$ & \begin{tabular}{|c|} 
PERMEATE \\
FLUX
\end{tabular} & \\
\hline $15: 04$ & $0: 00$ & 57 & 62 & 269 & 1499 & 227 & 162.9 & \\
\hline $15: 06$ & $0: 02$ & 52 & 62 & 269 & 1496 & 223 & 160.1 & \\
\hline $15: 08$ & $0: 04$ & 55 & 62 & 266 & 1500 & 221 & 158.6 & \\
\hline $15: 10$ & $0: 06$ & 51 & 62 & 267 & 1501 & 219 & 157.2 & \\
\hline $15: 12$ & $0: 08$ & 58 & 62 & 267 & 1493 & 216 & 155.1 & \\
\hline $15: 14$ & $0: 10$ & 55 & 61 & 268 & 1490 & 217 & 155.8 & \\
\hline $15: 16$ & $0: 12$ & 52 & 62 & 269 & 1499 & 214 & 153.6 & \\
\hline $15: 18$ & $0: 14$ & 65 & 62 & 265 & 1503 & 213 & 152.9 & \\
\hline $15: 20$ & $0: 16$ & 51 & 62 & 268 & 1489 & 212 & 152.2 & \\
\hline $15: 22$ & $0: 18$ & 59 & 62 & 266 & 1500 & 210 & 150.7 & \\
\hline $15: 24$ & $0: 20$ & 65 & 62 & 269 & 1494 & 210 & 150.7 & \\
\hline $15: 26$ & $0: 22$ & 58 & 61 & 270 & 1503 & 207 & 148.6 & \\
\hline $15: 28$ & $0: 24$ & 68 & 62 & 268 & 1502 & 208 & 149.3 & \\
\hline $15: 30$ & $0: 26$ & 64 & 62 & 263 & 1503 & 207 & 148.6 & \\
\hline $15: 32$ & $0: 28$ & 65 & 62 & 266 & 1499 & 203 & 145.7 & \\
\hline $15: 34$ & $0: 30$ & 55 & 62 & 268 & 1505 & 204 & 146.4 & \\
\hline $15: 36$ & $0: 32$ & 63 & 62 & 267 & 1503 & 202 & 145.0 & \\
\hline $15: 38$ & $0: 34$ & 55 & 62 & 263 & 1496 & 203 & 145.7 & \\
\hline $15: 40$ & $0: 36$ & 66 & 62 & 268 & 1498 & 200 & 143.6 & \\
\hline $15: 42$ & $0: 38$ & 52 & 62 & 264 & 1489 & 201 & 144.3 & \\
\hline $15: 44$ & $0: 40$ & 54 & 61 & 272 & 1498 & 203 & 145.7 & \\
\hline $15: 46$ & $0: 42$ & 67 & 62 & 268 & 1495 & 201 & 144.3 & \\
\hline $15: 48$ & $0: 44$ & 55 & 61 & 264 & 1496 & 197 & 141.4 & Begin Steady-state \\
\hline $15: 50$ & $0: 46$ & 58 & 60 & 268 & 1501 & 197 & 141.4 & \\
\hline $15: 52$ & $0: 48$ & 54 & 62 & 264 & 1497 & 200 & 143.6 & \\
\hline $15: 54$ & $0: 50$ & 63 & 61 & 268 & 1502 & 199 & 142.8 & \\
\hline $15: 56$ & $0: 52$ & 67 & 61 & 268 & 1500 & 199 & 142.8 & \\
\hline $15: 58$ & $0: 54$ & 67 & 61 & 263 & 1499 & 200 & 143.6 & \\
\hline $16: 00$ & $0: 56$ & 59 & 62 & 268 & 1499 & 197 & 141.4 & \\
\hline $16: 02$ & $0: 58$ & 58 & 61 & 267 & 1489 & 201 & 144.3 & \\
\hline $16: 04$ & $1: 00$ & 57 & 60 & 265 & 1498 & 199 & 142.8 & \\
\hline $16: 06$ & $1: 02$ & 55 & 61 & 270 & 1496 & 198 & 142.1 & \\
\hline $16: 08$ & $1: 04$ & 59 & 61 & 266 & 1498 & 197 & 141.4 & \\
\hline $16: 10$ & $1: 06$ & 56 & 61 & 266 & 1499 & 197 & 141.4 & \\
\hline $16: 12$ & $1: 08$ & 56 & 60 & 267 & 1501 & 195 & 140.0 & \\
\hline $16: 14$ & $1: 10$ & 59 & 61 & 262 & 1495 & 194 & 139.3 & \\
\hline $16: 16$ & $1: 12$ & 55 & 62 & 266 & 1493 & 196 & 140.7 & \\
\hline $16: 18$ & $1: 14$ & 65 & 62 & 266 & 1502 & 195 & 140.0 & \\
\hline $16: 20$ & $1: 16$ & 59 & 61 & 262 & 1496 & 198 & 142.1 & \\
\hline $16: 22$ & $1: 18$ & 59 & 60 & 271 & 1494 & 194 & 139.3 & \\
\hline $16: 24$ & $1: 20$ & 57 & 61 & 266 & 1503 & 196 & 140.7 & \\
\hline $16: 26$ & $1: 22$ & 55 & 61 & 266 & 1499 & 194 & 139.3 & \\
\hline $16: 28$ & $1: 24$ & 60 & 61 & 269 & 1496 & 197 & 141.4 & \\
\hline $16: 30$ & $1: 26$ & 68 & 60 & 268 & 1500 & 195 & 140.0 & \\
\hline $16: 32$ & $1: 28$ & 53 & 61 & 270 & 1492 & 193 & 138.5 & \\
\hline
\end{tabular}


MATR_21.XLS

\begin{tabular}{|c|c|c|c|c|c|c|c|c|}
\hline $\begin{array}{l}\text { TIME OF } \\
\text { DAY }\end{array}$ & $\begin{array}{c}\text { ELAPSED } \\
\text { TIME }\end{array}$ & \begin{tabular}{|c|} 
FEED \\
PRESSURE \\
\end{tabular} & FEED TEMP & FEED FLOW & $\begin{array}{l}\text { ROTOR } \\
\text { SPEED }\end{array}$ & $\begin{array}{c}\text { PERMEATE } \\
\text { FLOW }\end{array}$ & $\begin{array}{c}\text { PERMEATE } \\
\text { FLUX }\end{array}$ & \\
\hline $16: 34$ & $1: 30$ & 67 & 60 & 267 & 1490 & 194 & 139.3 & \\
\hline $16: 36$ & $1: 32$ & 66 & 60 & 265 & 1495 & 196 & 140.7 & \\
\hline $16: 38$ & $1: 34$ & 67 & 60 & 266 & 1497 & 197 & 141.4 & \\
\hline $16: 40$ & $1: 36$ & 62 & 60 & 263 & 1498 & 196 & 140.7 & \\
\hline $16: 42$ & $1: 38$ & 59 & 60 & 267 & 1497 & 194 & 139.3 & \\
\hline $16: 45$ & $1: 40$ & 53 & 61 & 263 & 1503 & 195 & 140.0 & \\
\hline $16: 47$ & $1: 42$ & 55 & 60 & 266 & 1500 & 194 & 139.3 & \\
\hline $16: 49$ & $1: 44$ & 56 & 60 & 265 & 1498 & 193 & 138.5 & \\
\hline $16: 51$ & $1: 46$ & 65 & 61 & 263 & 1501 & 194 & 139.3 & \\
\hline $16: 53$ & $1: 48$ & 68 & 61 & 269 & 1494 & 193 & 138.5 & \\
\hline $16: 55$ & $1: 50$ & 55 & 60 & 265 & 1496 & 191 & 137.1 & \\
\hline $16: 57$ & $1: 52$ & 54 & 61 & 265 & 1498 & 198 & 142.1 & \\
\hline $16: 59$ & $1: 54$ & 54 & 61 & 273 & 1497 & 192 & 137.8 & \\
\hline $17: 01$ & $1: 56$ & 57 & 61 & 269 & 1495 & 194 & 139.3 & \\
\hline $17: 03$ & $1: 58$ & 53 & 61 & 266 & 1500 & 196 & 140.7 & \\
\hline $17: 05$ & $2: 00$ & 53 & 61 & 268 & 1504 & 193 & 138.5 & \\
\hline $17: 07$ & $2: 02$ & 57 & 61 & 267 & 1503 & 193 & 138.5 & \\
\hline $17: 09$ & $2: 04$ & 59 & 61 & 267 & 1502 & 195 & 140.0 & \\
\hline $17: 11$ & $2: 06$ & 54 & 61 & 264 & 1497 & 193 & 138.5 & \\
\hline $17: 13$ & $2: 08$ & 60 & 61 & 267 & 1491 & 194 & 139.3 & \\
\hline $17: 15$ & $2: 10$ & 56 & 60 & 268 & 1498 & 196 & 140.7 & \\
\hline $17: 17$ & $2: 12$ & 59 & 61 & 266 & 1496 & 192 & 137.8 & \\
\hline $17: 19$ & $2: 14$ & 59 & 60 & 269 & 1496 & 191 & 137.1 & \\
\hline $17: 21$ & $2: 16$ & 67 & 63 & 263 & 1499 & 191 & 137.1 & \\
\hline $17: 23$ & $2: 18$ & 67 & 63 & 265 & 1501 & 193 & 138.5 & \\
\hline $17: 25$ & $2: 20$ & 54 & 62 & 270 & 1503 & 193 & 138.5 & \\
\hline $17: 27$ & $2: 22$ & 58 & 62 & 264 & 1494 & 193 & 138.5 & \\
\hline $17: 29$ & $2: 24$ & 56 & 62 & 262 & 1499 & 194 & 139.3 & \\
\hline $17: 31$ & $2: 26$ & 53 & 62 & 265 & 1504 & 192 & 137.8 & \\
\hline $17: 33$ & $2: 28$ & 58 & 62 & 269 & 1499 & 195 & 140.0 & \\
\hline $17: 35$ & $2: 30$ & 67 & 63 & 268 & 1501 & 192 & 137.8 & End Steady-state \\
\hline \multicolumn{2}{|c|}{ Steady-state Avg. } & 59 & 61 & 266 & 1498 & 195 & 140 & \\
\hline \multicolumn{2}{|c|}{ Maximum } & 68 & 63 & 273 & 1504 & 201 & 144 & \\
\hline Minimum & & 53 & 60 & 262 & 1489 & 191 & 137 & \\
\hline \multicolumn{2}{|c|}{ Standard Deviation } & 4.8 & 0.8 & 2.4 & 3.5 & 2.5 & 1.8 & \\
\hline \multicolumn{4}{|c|}{ TEST CONDITIONS: MATR_21.XLS } & Temp. $=$ & $60 \mathrm{~F}$ & & & \\
\hline & & & & Press. $=$ & $60 \mathrm{psig}$ & & & \\
\hline \multicolumn{3}{|c|}{ Run Date: 17-May-96 } & & Rotor $=$ & $1500 \mathrm{rpm}$ & & & \\
\hline & & & & Solids = & $1 w t \%$ & & & \\
\hline & & & & Flow $=$ & $400 \mathrm{~L} / \mathrm{hr}$ & & & \\
\hline
\end{tabular}


SpinTek II High Shear Rotary Membrane System

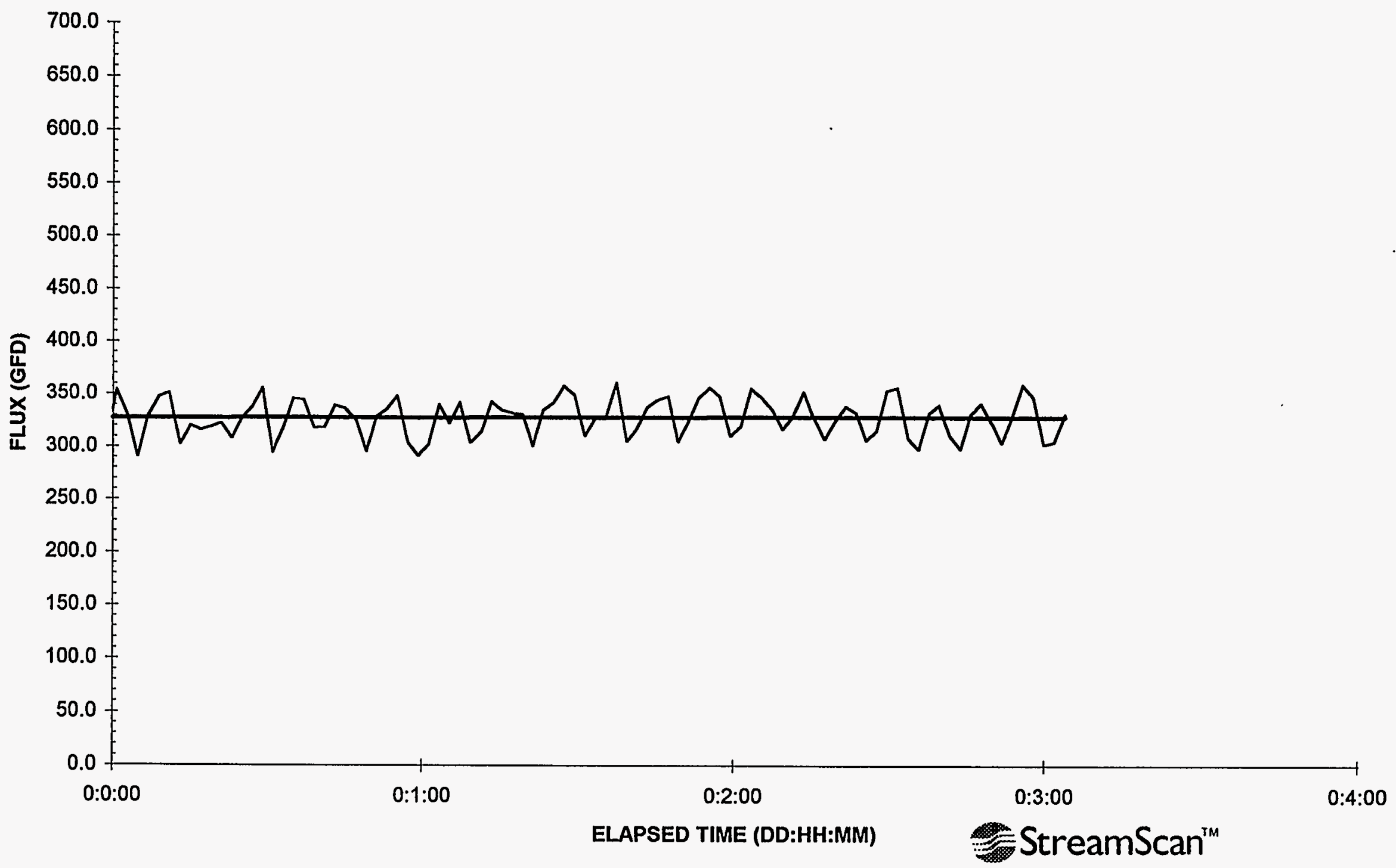




\begin{tabular}{|c|c|c|c|c|c|c|c|c|}
\hline $\begin{array}{l}\text { TIME OF } \\
\text { DAY }\end{array}$ & $\begin{array}{c}\text { ELAPSED } \\
\text { TIME }\end{array}$ & \begin{tabular}{c|} 
FEED \\
PRESSURE
\end{tabular} & FEED TEMP & FEED FLOW & $\begin{array}{l}\text { ROTOR } \\
\text { SPEED }\end{array}$ & $\begin{array}{l}\text { PERMEATE } \\
\text { FLOW }\end{array}$ & $\begin{array}{c}\text { PERMEATE } \\
\text { FLUX }\end{array}$ & \\
\hline $18: 31$ & $0: 00$ & 80 & 90 & 194 & 2010 & 467 & 335.2 & \\
\hline $18: 32$ & $0: 00$ & 77 & 90 & 193 & 2016 & 493 & 353.9 & \\
\hline $18: 34$ & $0: 02$ & 75 & 91 & 190 & 2016 & 461 & 330.9 & \\
\hline $18: 36$ & $0: 04$ & 77 & 89 & 242 & 2020 & 405 & 290.7 & \\
\hline $18: 38$ & $0: 06$ & 73 & 88 & 241 & 2008 & 459 & 329.5 & \\
\hline $18: 40$ & $0: 08$ & 70 & 90 & 241 & 2011 & 484 & 347.4 & \\
\hline $18: 42$ & $0: 10$ & 70 & 90 & 244 & 2016 & 489 & 351.0 & \\
\hline $18: 44$ & $0: 12$ & 70 & 90 & 240 & 2019 & 421 & 302.2 & \\
\hline $18: 46$ & $0: 14$ & 78 & 89 & 244 & 2016 & 446 & 320.2 & \\
\hline $18: 48$ & $0: 16$ & 84 & 88 & 240 & 2009 & 440 & 315.8 & \\
\hline $18: 50$ & $0: 18$ & 83 & 90 & 239 & 2012 & 444 & 318.7 & \\
\hline $18: 52$ & $0: 20$ & 84 & 91 & 243 & 2016 & 449 & 322.3 & \\
\hline $18: 54$ & $0: 22$ & 80 & 90 & 240 & 2014 & 429 & 307.9 & \\
\hline $18: 56$ & $0: 24$ & 71 & 88 & 241 & 2016 & 455 & 326.6 & \\
\hline $18: 58$ & $0: 26$ & 75 & 90 & 245 & 2009 & 471 & 338.1 & \\
\hline $19: 00$ & $0: 29$ & 83 & 91 & 244 & 2005 & 496 & 356.0 & \\
\hline $19: 02$ & $0: 31$ & 78 & 90 & 240 & 2012 & 410 & 294.3 & \\
\hline $19: 04$ & $0: 33$ & 71 & 89 & 239 & 2017 & 442 & 317.3 & Begin Steady-state \\
\hline $19: 06$ & $0: 34$ & 74 & 89 & 244 & 2010 & 482 & 346.0 & \\
\hline $19: 08$ & $0: 37$ & 75 & 90 & 241 & 2011 & 480 & 344.6 & \\
\hline $19: 10$ & $0: 39$ & 76 & 90 & 240 & 2015 & 443 & 318.0 & \\
\hline $19: 12$ & $0: 41$ & 77 & 89 & 243 & 2016 & $\overline{444}$ & 318.7 & \\
\hline $19: 14$ & $0: 43$ & 66 & 88 & 240 & 2009 & 473 & 339.5 & \\
\hline $19: 16$ & $0: 45$ & 69 & 90 & 241 & 2015 & 469 & 336.7 & \\
\hline $19: 18$ & $0: 47$ & 78 & 91 & 243 & 2011 & 453 & 325.2 & \\
\hline $19: 20$ & $0: 49$ & 84 & 91 & 240 & 2014 & 412 & 295.7 & \\
\hline $19: 22$ & $0: 51$ & 69 & 89 & 244 & 2012 & 458 & 328.8 & \\
\hline $19: 24$ & $0: 53$ & 77 & 89 & 247 & 2010 & 468 & 335.9 & \\
\hline $19: 26$ & $0: 55$ & 69 & 91 & 243 & 2014 & 485 & 348.1 & \\
\hline $19: 28$ & $0: 57$ & 78 & 93 & 243 & 2024 & 424 & 304.4 & \\
\hline $19: 30$ & $0: 59$ & 73 & 91 & 237 & 2013 & 406 & 291.4 & \\
\hline $19: 32$ & $1: 01$ & 69 & 89 & 238 & 2020 & 421 & 302.2 & \\
\hline $19: 34$ & $1: 03$ & 76 & 88 & 240 & 2009 & 474 & 340.3 & \\
\hline $19: 36$ & $1: 05$ & 68 & 89 & 239 & 2013 & 449 & 322.3 & \\
\hline $19: 38$ & $1: 07$ & 73 & 90 & 237 & 2016 & 477 & 342.4 & \\
\hline $19: 40$ & $1: 09$ & 66 & 90 & 240 & 2011 & 424 & 304.4 & \\
\hline $19: 42$ & $1: 11$ & 76 & 89 & 240 & 2016 & 438 & 314.4 & \\
\hline $19: 44$ & $1: 13$ & 69 & 88 & 239 & 2016 & 478 & 343.1 & \\
\hline $19: 46$ & $1: 15$ & 71 & 89 & 238 & 2025 & 467 & 335.2 & \\
\hline $19: 48$ & $1: 17$ & 70 & 90 & 242 & 2016 & 463 & 332.4 & \\
\hline $19: 50$ & $1: 19$ & 66 & 91 & 239 & 2023 & 461 & 330.9 & \\
\hline $19: 52$ & $1: 21$ & 67 & 90 & 241 & 2003 & 419 & 300.8 & \\
\hline $19: 54$ & $1: 23$ & 69 & 88 & 244 & 2010 & 467 & 335.2 & \\
\hline $19: 56$ & $1: 25$ & 71 & 90 & 244 & 2011 & 477 & 342.4 & \\
\hline $19: 58$ & $1: 27$ & 84 & 90 & 244 & 2026 & 499 & 358.2 & \\
\hline
\end{tabular}


MATR_22.XLS

\begin{tabular}{|c|c|c|c|c|c|c|c|c|}
\hline $\begin{array}{c}\text { TIME OF } \\
\text { DAY }\end{array}$ & $\begin{array}{c}\text { ELAPSED } \\
\text { TIME }\end{array}$ & \begin{tabular}{c|} 
FEED \\
PRESSURE
\end{tabular} & FEED TEMP & FEED FLOW & $\begin{array}{l}\text { ROTOR } \\
\text { SPEED }\end{array}$ & $\begin{array}{l}\text { PERMEATE } \\
\text { FLOW }\end{array}$ & $\begin{array}{l}\text { PERMEATE } \\
\text { FLUX }\end{array}$ & \\
\hline $20: 00$ & $1: 29$ & 76 & 91 & 244 & 2002 & 487 & 349.6 & \\
\hline $20: 02$ & $1: 31$ & 69 & 90 & 237 & 2014 & 433 & 310.8 & \\
\hline $20: 04$ & $1: 33$ & 83 & 89 & 245 & 2013 & 456 & 327.3 & \\
\hline $20: 06$ & $1: 35$ & 72 & 89 & 241 & 2015 & 455 & 326.6 & \\
\hline $20: 08$ & $1: 37$ & 68 & 90 & 241 & 2016 & 503 & 361.1 & \\
\hline $20: 10$ & $1: 39$ & 75 & 91 & 245 & 2019 & 426 & 305.8 & \\
\hline $20: 12$ & $1: 41$ & 78 & 89 & 242 & 2018 & 442 & 317.3 & \\
\hline $20: 14$ & $1: 43$ & 72 & 88 & 238 & 2010 & 471 & 338.1 & \\
\hline $20: 16$ & $1: 45$ & 66 & 89 & 245 & 2017 & 481 & 345.3 & \\
\hline $20: 18$ & $1: 47$ & 72 & 90 & 242 & 2017 & 485 & 348.1 & \\
\hline $20: 20$ & $1: 49$ & 66 & 90 & 237 & 2010 & 425 & 305.1 & \\
\hline $20: 22$ & $1: 51$ & 72 & 90 & 243 & 2016 & 452 & 324.5 & \\
\hline $20: 24$ & $1: 53$ & 69 & 88 & 243 & 2017 & 483 & 346.7 & \\
\hline $20: 26$ & $1: 55$ & 69 & 90 & 241 & 2011 & 497 & 356.8 & \\
\hline $20: 28$ & $1: 57$ & 71 & 90 & 244 & 2008 & 485 & 348.1 & \\
\hline $20: 30$ & $1: 59$ & 73 & 91 & 247 & 2014 & 433 & 310.8 & \\
\hline $20: 32$ & $2: 01$ & 77 & 88 & 241 & 2014 & 446 & 320.2 & \\
\hline $20: 34$ & $2: 03$ & 67 & 88 & 240 & 2011 & 496 & 356.0 & \\
\hline $20: 36$ & $2: 05$ & 82 & 89 & 244 & 2010 & 484 & 347.4 & \\
\hline $20: 38$ & $2: 07$ & 72 & 91 & 243 & 2017 & 468 & 335.9 & \\
\hline $20: 41$ & $2: 09$ & 74 & 90 & 244 & 2014 & 442 & 317.3 & \\
\hline $20: 43$ & $2: 11$ & 82 & 89 & 241 & 2024 & 459 & 329.5 & \\
\hline $20: 45$ & $2: 13$ & 67 & 89 & 239 & 2016 & 491 & 352.5 & \\
\hline $20: 47$ & $2: 15$ & 84 & 91 & 241 & 2016 & 456 & 327.3 & \\
\hline $20: 49$ & $2: 17$ & 70 & 91 & 236 & 2018 & 429 & 307.9 & \\
\hline $20: 51$ & $2: 19$ & 69 & 90 & 246 & 2022 & 452 & 324.5 & \\
\hline $20: 53$ & $2: 21$ & 84 & 89 & 244 & 2010 & 472 & 338.8 & \\
\hline $20: 55$ & $2: 23$ & 77 & 91 & 243 & 2019 & 464 & 333.1 & \\
\hline $20: 57$ & $2: 25$ & 72 & 91 & 243 & 2015 & 427 & 306.5 & \\
\hline $20: 59$ & $2: 27$ & 73 & 89 & 241 & 2016 & 440 & 315.8 & \\
\hline $21: 01$ & $2: 29$ & 76 & 89 & 237 & 2017 & 493 & 353.9 & \\
\hline $21: 03$ & $2: 31$ & 71 & 90 & 244 & 2019 & 497 & 356.8 & \\
\hline $21: 05$ & $2: 33$ & 77 & 92 & 237 & 2010 & 430 & 308.7 & \\
\hline $21: 07$ & $2: 35$ & 69 & 91 & 241 & 2018 & 415 & 297.9 & \\
\hline $21: 09$ & $2: 37$ & 70 & 88 & 242 & 2019 & 463 & 332.4 & \\
\hline $21: 11$ & $2: 39$ & 78 & 89 & 242 & 2010 & 474 & 340.3 & \\
\hline $21: 13$ & $2: 41$ & 74 & 91 & 241 & 2016 & 434 & 311.5 & \\
\hline $21: 15$ & $2: 43$ & 70 & 90 & 241 & 2008 & 416 & 298.6 & \\
\hline $21: 17$ & $2: 45$ & 71 & 89 & 244 & 2021 & 462 & 331.6 & \\
\hline $21: 19$ & $2: 47$ & 77 & 90 & 241 & 2029 & 476 & 341.7 & \\
\hline $21: 21$ & $2: 49$ & 71 & 92 & 242 & 2003 & 451 & 323.7 & \\
\hline $21: 23$ & $2: 51$ & 68 & 91 & 240 & 2010 & 424 & 304.4 & \\
\hline $21: 25$ & $2: 53$ & 76 & 89 & 240 & 2016 & 458 & 328.8 & \\
\hline $21: 27$ & $2: 55$ & 76 & 89 & 244 & 2010 & 501 & 359.6 & \\
\hline $21: 29$ & $2: 57$ & 70 & 91 & 245 & 2008 & 485 & 348.1 & \\
\hline $21: 31$ & $2: 59$ & 69 & 91 & 239 & 2016 & 422 & 302.9 & \\
\hline $21: 33$ & $3: 01$ & 66 & 90 & 240 & 2014 & 425 & 305.1 & \\
\hline $21: 35$ & $3: 04$ & 74 & 89 & 245 & 2016 & 462 & 331.6 & End Steady-state \\
\hline
\end{tabular}


MATR_22.XLS

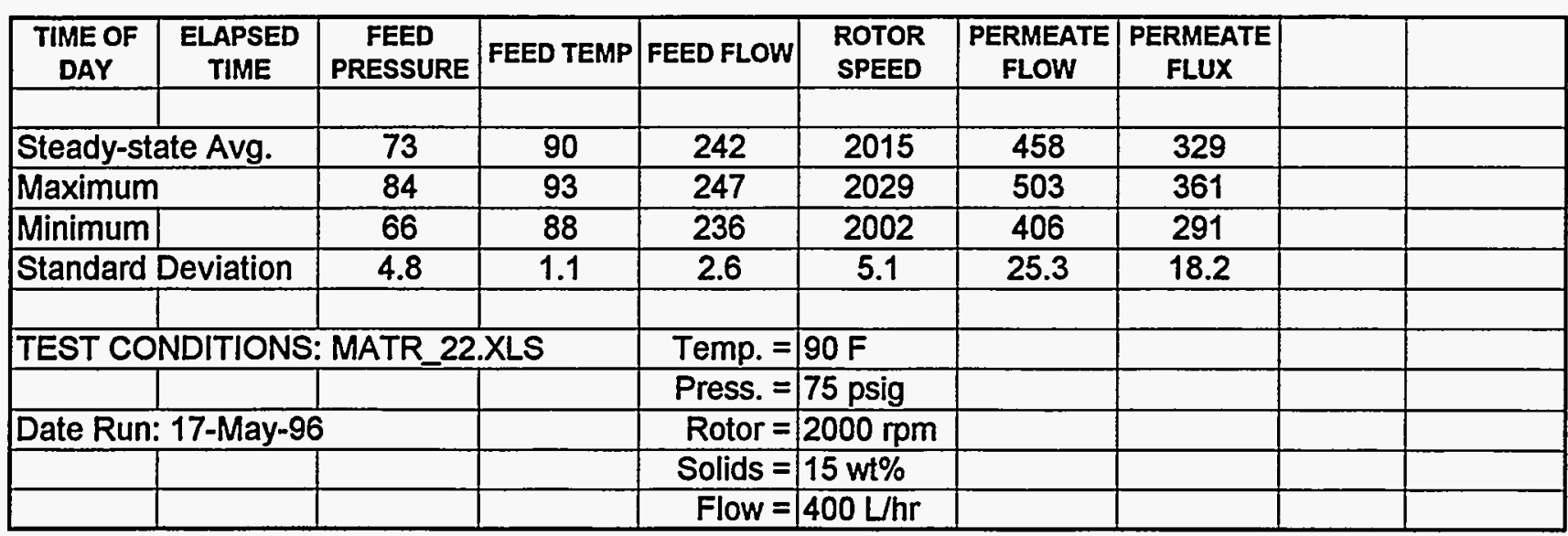


SpinTek II High Shear Rotary Membrane System

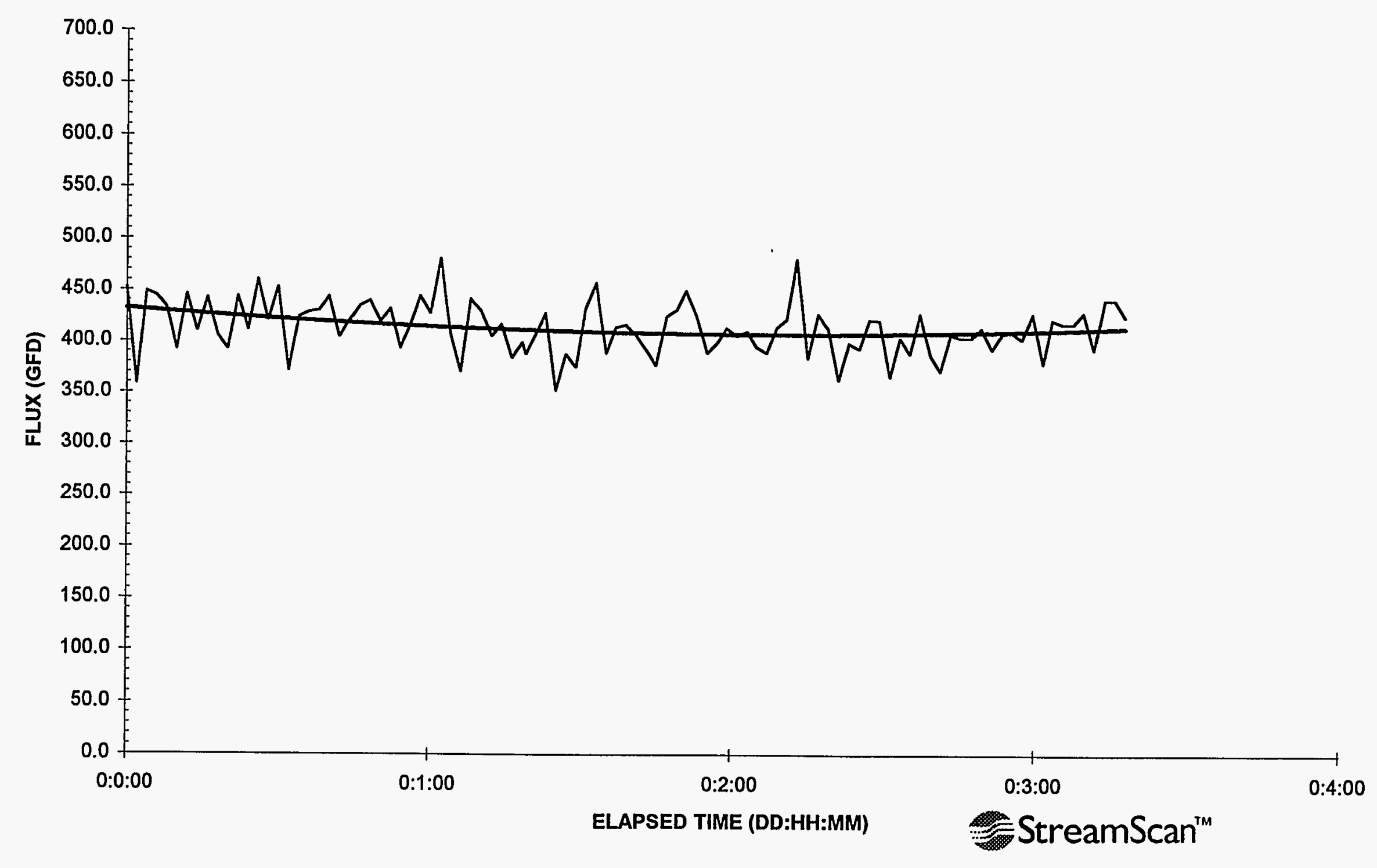




\begin{tabular}{|c|c|c|c|c|c|c|c|c|}
\hline $\begin{array}{l}\text { TIME OF } \\
\text { DAY }\end{array}$ & $\begin{array}{c}\text { ELAPSED } \\
\text { TIME }\end{array}$ & $\begin{array}{c}\text { FEED } \\
\text { PRESSURE }\end{array}$ & FEED TEMP & FEED FLOW & $\begin{array}{l}\text { ROTOR } \\
\text { SPEED }\end{array}$ & $\begin{array}{l}\text { PERMEATE } \\
\text { FLOW }\end{array}$ & $\begin{array}{c}\text { PERMEATE } \\
\text { FLUX }\end{array}$ & \\
\hline $22: 40$ & $0: 00$ & 56 & 120 & 420 & 1991 & 630 & 452.2 & \\
\hline $22: 42$ & $0: 02$ & 57 & 120 & 399 & 1990 & 500 & 358.9 & \\
\hline $22: 44$ & $0: 04$ & 52 & 117 & 418 & 1990 & 625 & 448.6 & \\
\hline $22: 46$ & $0: 06$ & 52 & 117 & $\overline{418}$ & 1991 & 619 & 444.3 & \\
\hline $22: 48$ & $0: 08$ & 59 & 117 & 412 & 1992 & 603 & 432.9 & \\
\hline $22: 50$ & $0: 10$ & 62 & 118 & 417 & 1992 & 546 & 391.9 & \\
\hline $22: 52$ & $0: 12$ & 54 & 118 & 432 & 1996 & 621 & 445.8 & \\
\hline $22: 55$ & $0: 14$ & 56 & 119 & 440 & 1988 & 572 & 410.6 & \\
\hline $22: 57$ & $0: 16$ & 52 & 120 & 428 & 1987 & 616 & 442.2 & \\
\hline $22: 59$ & $0: 18$ & 57 & $\overline{121}$ & 417 & 1989 & 566 & 406.3 & \\
\hline $23: 01$ & $0: 20$ & 62 & 121 & 430 & 1988 & 547 & 392.7 & \\
\hline $23: 03$ & $0: 22$ & 59 & 118 & 414 & 2000 & 618 & 443.6 & \\
\hline 23:05 & $0: 24$ & 54 & 118 & 421 & 2002 & 573 & 411.3 & \\
\hline $23: 07$ & $0: 26$ & 53 & 119 & 416 & 1989 & 641 & 460.1 & \\
\hline $23: 09$ & $0: 28$ & 52 & 120 & 423 & 1989 & 586 & 420.6 & \\
\hline $23: 11$ & $0: 30$ & 67 & 121 & 425 & 1994 & 631 & 452.9 & \\
\hline $23: 13$ & $0: 32$ & 59 & 120 & 436 & 1992 & 518 & 371.8 & \\
\hline $23: 15$ & $0: 34$ & 55 & 118 & 407 & 1986 & 591 & 424.2 & \\
\hline $23: 17$ & $0: 36$ & 54 & 119 & 408 & 1989 & 597 & 428.5 & \\
\hline $23: 19$ & $0: 38$ & 57 & 120 & 422 & 1989 & 600 & 430.7 & \\
\hline $23: 21$ & $0: 40$ & 54 & 120 & 421 & 1996 & 618 & 443.6 & \\
\hline $23: 23$ & $0: 42$ & 57 & 122 & 424 & 1994 & 564 & 404.9 & \\
\hline $23: 25$ & $0: 44$ & 64 & 118 & 424 & 1989 & 587 & 421.4 & \\
\hline $23: 27$ & $0: 46$ & 61 & 118 & 419 & 2003 & 606 & 435.0 & \\
\hline $23: 29$ & $0: 48$ & 65 & 119 & 415 & 1987 & 613 & 440.0 & \\
\hline $23: 31$ & $0: 50$ & 58 & 120 & 414 & 2002 & 585 & 419.9 & \\
\hline $23: 33$ & $0: 52$ & 56 & 121 & 419 & 1988 & 602 & 432.1 & \\
\hline $23: 35$ & $0: 54$ & 58 & 121 & 420 & 1991 & 548 & 393.4 & \\
\hline $23: 37$ & $0: 56$ & 54 & 119 & 434 & 1997 & 580 & 416.3 & \\
\hline $23: 39$ & $0: 58$ & 53 & 119 & 427 & 1992 & 619 & 444.3 & \\
\hline $23: 41$ & $1: 00$ & 67 & 120 & 421 & 1994 & 596 & 427.8 & \\
\hline $23: 43$ & $1: 02$ & 54 & 121 & 429 & 1995 & 670 & 480.9 & \\
\hline $23: 45$ & $1: 04$ & 60 & 124 & 419 & 1991 & 566 & 406.3 & \\
\hline $23: 47$ & $1: 06$ & 54 & 123 & 422 & 1989 & 517 & 371.1 & \\
\hline $23: 49$ & $1: 08$ & 54 & 118 & 419 & 1989 & 615 & 441.5 & \\
\hline $23: 51$ & $1: 10$ & 61 & 119 & 401 & 1989 & 600 & 430.7 & \\
\hline $23: 53$ & $1: 12$ & 52 & 120 & 414 & 1989 & 565 & 405.6 & \\
\hline $23: 55$ & $1: 14$ & 52 & 121 & 426 & 1989 & 581 & 417.1 & \\
\hline $23: 57$ & $1: 16$ & 53 & 122 & 411 & 2000 & 536 & 384.8 & Beqin Steady-state \\
\hline $23: 59$ & $1: 18$ & 56 & 119 & 397 & 1996 & 556 & 399.1 & \\
\hline $0: 00$ & $1: 19$ & 55 & 118 & 411 & 1993 & 541 & 388.3 & \\
\hline $0: 02$ & $1: 21$ & 64 & 119 & 402 & 1989 & 568 & 407.7 & \\
\hline $0: 04$ & $1: 23$ & 52 & 120 & 424 & 1999 & 596 & 427.8 & \\
\hline $0: 06$ & $1: 25$ & 61 & 122 & 426 & 1989 & 491 & 352.5 & \\
\hline $0: 08$ & $1: 27$ & 59 & 118 & 409 & 1992 & 540 & 387.6 & \\
\hline
\end{tabular}


MATR_23.XLS

\begin{tabular}{|c|c|c|c|c|c|c|c|c|c|}
\hline $\begin{array}{l}\text { TIME OF } \\
\text { DAY }\end{array}$ & $\begin{array}{c}\text { ELAPSED } \\
\text { TIME }\end{array}$ & $\begin{array}{c}\text { FEED } \\
\text { PRESSURE } \\
\end{array}$ & FEED TEMP & FEED FLOW & $\begin{array}{l}\text { ROTOR } \\
\text { SPEED } \\
\end{array}$ & $\begin{array}{l}\text { PERMEATE } \\
\text { FLOW }\end{array}$ & $\begin{array}{c}\text { PERMEATE } \\
\text { FLUX }\end{array}$ & & \\
\hline $0: 10$ & $1: 29$ & $\overline{71}$ & 118 & 402 & 2002 & 523 & 375.4 & & \\
\hline $0: 12$ & $1: 31$ & 66 & 119 & 383 & 2003 & 602 & 432.1 & & \\
\hline $0: 14$ & $1: 33$ & 65 & 120 & 394 & 2003 & 637 & 457.3 & & \\
\hline $0: 16$ & $1: 35$ & 59 & 122 & 386 & 1993 & 542 & 389.1 & & \\
\hline $0: 18$ & $1: 37$ & 60 & 118 & 396 & 1995 & 577 & 414.2 & & \\
\hline $0: 20$ & $1: 39$ & 56 & 118 & 392 & 1997 & 581 & 417.1 & & \\
\hline $0: 22$ & $1: 41$ & 64 & 119 & 387 & 1989 & 567 & 407.0 & & \\
\hline $0: 24$ & $1: 43$ & 63 & 121 & 401 & 1997 & 547 & 392.7 & & \\
\hline $0: 26$ & $1: 45$ & 61 & 122 & 383 & 1989 & 526 & 377.6 & & \\
\hline $0: 28$ & $1: 47$ & 57 & 118 & 387 & 1996 & 592 & 425.0 & & \\
\hline $0: 30$ & $1: 49$ & 63 & 119 & 383 & 2003 & 601 & 431.4 & & \\
\hline $0: 32$ & $1: 51$ & 65 & 119 & 388 & 1989 & 627 & 450.1 & & \\
\hline $0: 34$ & $1: 53$ & 59 & 121 & 394 & 1991 & 594 & 426.4 & & \\
\hline $0: 36$ & $1: 55$ & 71 & 121 & 384 & 1992 & 543 & 389.8 & & \\
\hline $0: 38$ & $1: 57$ & 66 & 119 & 390 & 1987 & 556 & 399.1 & & \\
\hline $0: 40$ & $1: 59$ & 69 & 118 & 391 & 2000 & 576 & 413.5 & & \\
\hline $0: 42$ & $2: 01$ & 64 & 119 & 397 & 1989 & 566 & 406.3 & & \\
\hline $0: 44$ & $2: 03$ & 61 & 120 & 383 & 1996 & 572 & 410.6 & & \\
\hline $0: 46$ & $2: 05$ & 59 & 121 & 384 & 1991 & 551 & 395.5 & & \\
\hline $0: 48$ & $2: 07$ & 68 & 119 & 389 & 1999 & 543 & 389.8 & & \\
\hline $0: 50$ & $2: 09$ & 65 & 118 & 397 & 1989 & 577 & 414.2 & & \\
\hline $0: 52$ & $2: 11$ & 66 & 120 & 372 & 1990 & 589 & 422.8 & & \\
\hline $0: 54$ & $2: 13$ & 70 & 121 & 396 & 1991 & 670 & 480.9 & & \\
\hline $0: 56$ & $2: 15$ & 67 & 121 & 396 & 1992 & 536 & 384.8 & & \\
\hline $0: 58$ & $2: 17$ & 66 & 120 & 391 & 1990 & 595 & 427.1 & & \\
\hline $1: 00$ & $2: 19$ & 60 & 121 & 395 & 1990 & 576 & 413.5 & & \\
\hline $1: 02$ & $2: 21$ & 58 & 121 & 398 & 1998 & 506 & 363.2 & & \\
\hline $1: 04$ & $2: 23$ & 70 & 119 & 382 & 1993 & 556 & 399.1 & & \\
\hline $1: 06$ & $2: 25$ & 57 & 119 & 391 & 1991 & 549 & 394.1 & & \\
\hline $1: 08$ & $2: 27$ & 65 & 120 & 389 & 1992 & 588 & 422.1 & & \\
\hline $1: 10$ & $2: 29$ & 65 & 122 & 385 & 1992 & 587 & 421.4 & & \\
\hline $1: 12$ & $2: 31$ & 60 & 120 & 386 & 1988 & 511 & 366.8 & & \\
\hline $1: 14$ & $2: 33$ & 66 & 119 & 393 & 1991 & 563 & 404.1 & & \\
\hline $1: 16$ & $2: 35$ & 64 & 120 & 393 & 1995 & 542 & 389.1 & & \\
\hline $1: 18$ & $2: 37$ & 57 & 120 & 388 & 1993 & 596 & 427.8 & & \\
\hline $1: 20$ & $2: 39$ & 57 & 121 & 379 & 2007 & 540 & 387.6 & & \\
\hline $1: 22$ & $2: 41$ & 57 & 119 & 388 & 1989 & 519 & 372.6 & & \\
\hline $1: 24$ & $2: 43$ & 65 & 119 & 386 & 1990 & 568 & 407.7 & & \\
\hline $1: 26$ & $2: 45$ & 68 & 119 & 395 & 2000 & 564 & 404.9 & & \\
\hline $1: 28$ & $2: 47$ & 70 & 120 & 378 & 1994 & 564 & 404.9 & & \\
\hline $1: 30$ & $2: 49$ & 64 & 121 & 367 & 1996 & 576 & 413.5 & & \\
\hline $1: 32$ & $2: 51$ & 66 & 119 & 392 & 1989 & 548 & 393.4 & & \\
\hline $1: 34$ & $2: 53$ & 56 & 118 & 387 & 1993 & 569 & 408.4 & & \\
\hline $1: 36$ & $2: 55$ & 69 & 120 & 389 & 1998 & 572 & 410.6 & & \\
\hline $1: 38$ & $2: 57$ & 65 & 120 & 388 & 1999 & 562 & 403.4 & & \\
\hline $1: 40$ & $2: 59$ & 59 & 121 & 390 & 1993 & 596 & 427.8 & & \\
\hline $1: 42$ & $3: 01$ & 71 & 122 & 395 & 1989 & 529 & 379.7 & & \\
\hline $1: 44$ & $3: 03$ & 65 & 119 & 389 & 1995 & 588 & 422.1 & & \\
\hline $1: 46$ & $3: 05$ & 60 & 119 & 400 & 1992 & 583 & 418.5 & & \\
\hline $1: 48$ & $3: 07$ & 64 & 121 & 397 & 1999 & 583 & 418.5 & & \\
\hline
\end{tabular}


MATR_23.XLS

\begin{tabular}{|}
\begin{tabular}{|c|c|c|c|c|c|c|c|c|c|}
\hline $\begin{array}{c}\text { TIME OF } \\
\text { DAY }\end{array}$ & $\begin{array}{c}\text { ELAPSED } \\
\text { TIME }\end{array}$ & $\begin{array}{c}\text { FEED } \\
\text { PRESSURE }\end{array}$ & FEED TEMP & FEED FLOW & $\begin{array}{c}\text { ROTOR } \\
\text { SPEED }\end{array}$ & $\begin{array}{c}\text { PERMEATE } \\
\text { FLOW }\end{array}$ & $\begin{array}{c}\text { PERMEATE } \\
\text { FLUX }\end{array}$ & & \\
\hline $1: 50$ & $3: 09$ & 59 & 122 & 387 & 1989 & 598 & 429.3 & & \\
\hline $1: 52$ & $3: 11$ & 70 & 119 & 389 & 1989 & 548 & 393.4 & & \\
\hline $1: 54$ & $3: 13$ & 64 & 118 & 393 & 1991 & 615 & 441.5 & & \\
\hline $1: 56$ & $3: 15$ & 59 & 120 & 388 & 2002 & 615 & 441.5 & & \\
\hline $1: 58$ & $3: 17$ & 59 & 120 & 392 & 1997 & 593 & 425.7 & End Steady-state \\
\hline & & & & & & & & & \\
\hline
\end{tabular} Steady-state Avg. & 63 & 120 & 392 & 1994 & 568 & 408 & & \\
\hline Maximum & 71 & 122 & 426 & 2007 & 670 & 481 & & \\
\hline Minimum & 52 & 118 & 367 & 1987 & 491 & 352 & & \\
\hline Standard Deviation & 4.8 & 1.3 & 10.0 & 4.7 & 32.4 & 23.2 & & \\
\hline \multicolumn{7}{|l|}{} & & & & & & & & \\
\hline
\end{tabular}




\section{SpinTek II High Shear Rotary Membrane System}

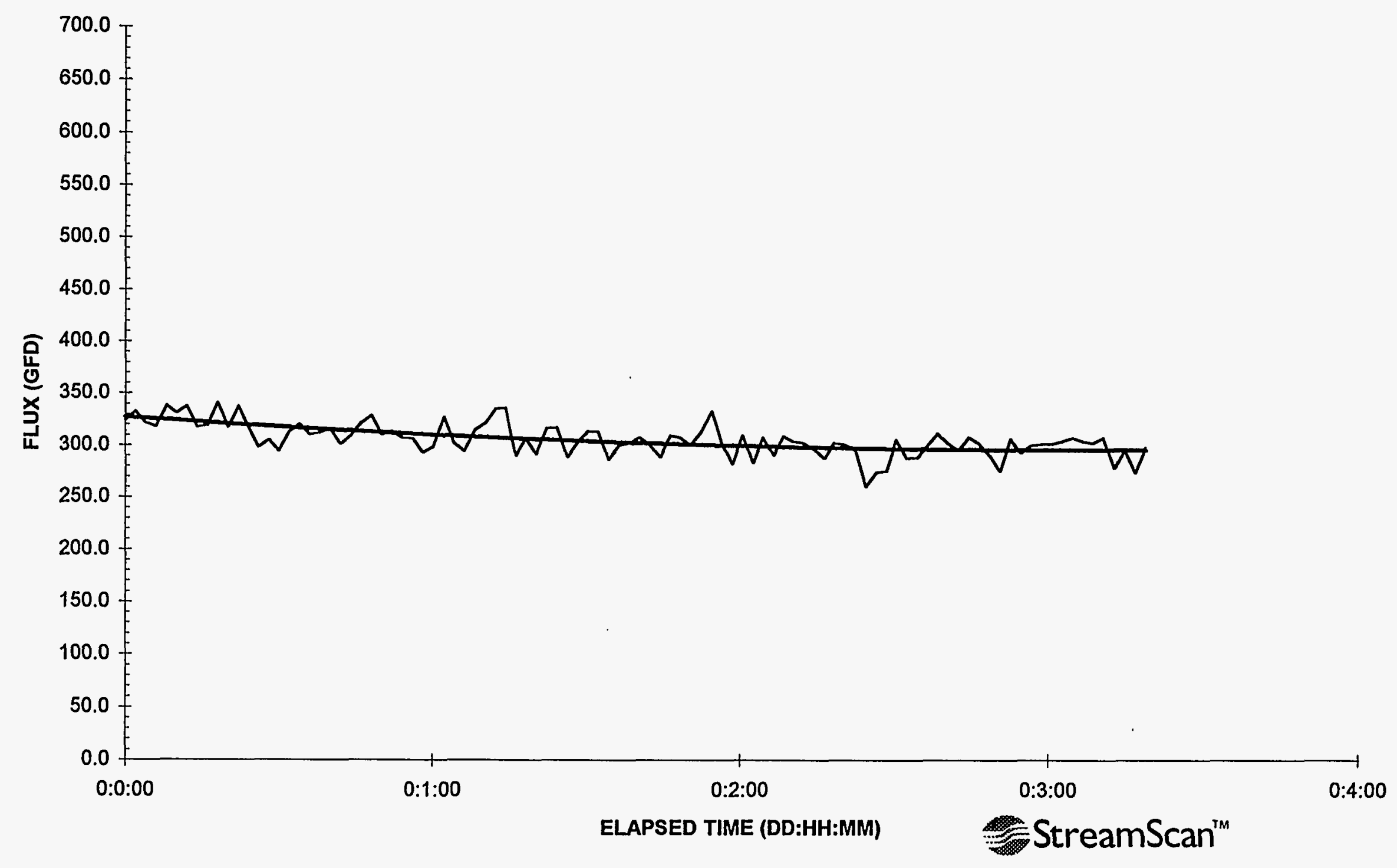




\begin{tabular}{|c|c|c|c|c|c|c|c|c|}
\hline $\begin{array}{c}\text { TIME OF } \\
\text { DAY }\end{array}$ & $\begin{array}{l}\text { ELAPSED } \\
\text { TIME }\end{array}$ & $\begin{array}{c}\text { FEED } \\
\text { PRESSURE }\end{array}$ & FEED TEMP & FEED FLOW & $\begin{array}{l}\text { ROTOR } \\
\text { SPEED }\end{array}$ & $\begin{array}{c}\text { PERMEATE } \\
\text { FLOW }\end{array}$ & $\begin{array}{c}\text { PERMEATE } \\
\text { FLUX }\end{array}$ & \\
\hline $2: 35$ & $0: 00$ & 43 & 120 & 396 & 1519 & 451 & 323.7 & \\
\hline $2: 37$ & $0: 01$ & 51 & 121 & 388 & 1507 & 463 & 332.4 & \\
\hline $2: 39$ & $0: 03$ & 50 & 119 & 394 & 1510 & 448 & 321.6 & \\
\hline $2: 41$ & $0: 05$ & 48 & 117 & 396 & 1505 & 443 & 318.0 & \\
\hline $2: 43$ & $0: 07$ & 42 & 118 & 399 & 1520 & 471 & 338.1 & \\
\hline $2: 45$ & $0: 09$ & 42 & 118 & 378 & 1513 & 461 & 330.9 & \\
\hline $2: 47$ & $0: 11$ & 53 & 118 & 395 & 1514 & 470 & 337.4 & \\
\hline $2: 49$ & $0: 13$ & 49 & 118 & 395 & 1508 & 442 & 317.3 & \\
\hline $2: 51$ & $0: 15$ & 50 & 119 & 383 & 1519 & 445 & 319.4 & \\
\hline $2: 53$ & $0: 17$ & 46 & 119 & 392 & 1515 & 475 & 341.0 & \\
\hline $2: 55$ & $0: 20$ & 44 & 120 & 389 & 1520 & 442 & 317.3 & \\
\hline $2: 57$ & $0: 22$ & 49 & 120 & 409 & 1515 & 470 & 337.4 & \\
\hline $2: 59$ & $0: 24$ & 43 & 121 & 390 & 1522 & 443 & 318.0 & \\
\hline $3: 01$ & $0: 26$ & 47 & 120 & 390 & 1521 & 415 & 297.9 & \\
\hline $3: 03$ & $0: 28$ & 53 & 118 & 380 & 1505 & 425 & 305.1 & \\
\hline $3: 05$ & $0: 30$ & 44 & 118 & 378 & 1517 & 410 & 294.3 & \\
\hline 3:07 & $0: 32$ & 51 & 119 & 382 & 1512 & 436 & 313.0 & \\
\hline $3: 09$ & $0: 34$ & 43 & 119 & 385 & 1510 & 446 & 320.2 & \\
\hline $3: 11$ & $0: 36$ & 48 & 119 & 378 & 1507 & 432 & 310.1 & \\
\hline $3: 13$ & $0: 38$ & 43 & 119 & 378 & 1509 & 435 & 312.3 & \\
\hline $3: 15$ & $0: 40$ & 46 & 119 & 378 & 1516 & 440 & 315.8 & \\
\hline $3: 17$ & $0: 42$ & 52 & 120 & 378 & 1513 & 420 & 301.5 & \\
\hline $3: 19$ & $0: 44$ & 46 & 120 & 379 & 1514 & 430 & 308.7 & \\
\hline $3: 21$ & $0: 46$ & 53 & 120 & 398 & 1513 & 448 & 321.6 & \\
\hline $3: 23$ & $0: 48$ & 42 & 120 & 402 & 1521 & 458 & 328.8 & \\
\hline $3: 25$ & $0: 50$ & 53 & 120 & 387 & 1512 & 432 & 310.1 & \\
\hline $3: 27$ & $0: 52$ & 44 & 121 & 403 & 1512 & 437 & 313.7 & \\
\hline $3: 29$ & $0: 54$ & 45 & 121 & 392 & 1521 & 428 & 307.2 & \\
\hline $3: 32$ & $0: 56$ & 42 & 120 & 402 & 1517 & 427 & 306.5 & \\
\hline $3: 34$ & $0: 58$ & 42 & 119 & 387 & 1507 & 408 & 292.9 & \\
\hline $3: 36$ & $1: 00$ & 42 & 119 & 399 & 1507 & 416 & 298.6 & \\
\hline $3: 38$ & $1: 02$ & 49 & 119 & 388 & 1512 & 456 & 327.3 & \\
\hline $3: 40$ & $1: 04$ & 42 & 120 & 387 & 1507 & 421 & 302.2 & \\
\hline $3: 42$ & $1: 06$ & 47 & 120 & 391 & 1515 & 410 & 294.3 & \\
\hline $3: 44$ & $1: 08$ & 45 & 120 & 386 & 1509 & 439 & 315.1 & \\
\hline $3: 46$ & $1: 10$ & 46 & 121 & 404 & 1513 & 448 & 321.6 & \\
\hline $3: 48$ & $1: 12$ & 44 & 121 & 393 & 1501 & 467 & 335.2 & \\
\hline $3: 50$ & $1: 14$ & 44 & 121 & 391 & 1500 & 468 & 335.9 & \\
\hline $3: 52$ & $1: 16$ & 47 & 120 & 393 & 1508 & 404 & 290.0 & Begin Steady-state \\
\hline $3: 54$ & $1: 18$ & 44 & 119 & 387 & 1505 & 427 & 306.5 & \\
\hline $3: 56$ & $1: 20$ & 47 & 119 & 383 & 1503 & 406 & 291.4 & \\
\hline $3: 58$ & $1: 22$ & 44 & 119 & 392 & 1510 & 441 & 316.6 & \\
\hline $4: 00$ & $1: 24$ & 44 & 120 & 387 & 1502 & 442 & 317.3 & \\
\hline $4: 02$ & $1: 26$ & 44 & 120 & 397 & 1517 & 402 & 288.6 & \\
\hline $4: 04$ & $1: 28$ & 44 & 120 & 387 & 1500 & 421 & 302.2 & \\
\hline
\end{tabular}


MATR_24.XLS

\begin{tabular}{|c|c|c|c|c|c|c|c|c|c|}
\hline $\begin{array}{c}\text { TIME OF } \\
\text { DAY } \\
\end{array}$ & $\begin{array}{c}\text { ELAPSED } \\
\text { TIME }\end{array}$ & \begin{tabular}{c|} 
FEED \\
PRESSURE \\
\end{tabular} & FEED TEMP & FEED FLOW & $\begin{array}{l}\text { ROTOR } \\
\text { SPEED } \\
\end{array}$ & $\begin{array}{c}\text { PERMEATE } \\
\text { FLOW }\end{array}$ & $\begin{array}{c}\text { PERMEATE } \\
\text { FLUX }\end{array}$ & & \\
\hline $4: 06$ & $1: 30$ & 53 & 121 & 393 & 1507 & 437 & 313.7 & & \\
\hline $4: 08$ & $1: 32$ & 45 & 120 & 390 & 1505 & 436 & 313.0 & & \\
\hline $4: 10$ & $1: 34$ & 44 & 121 & 379 & 1499 & 399 & 286.4 & & \\
\hline $4: 12$ & $1: 36$ & 49 & 118 & 390 & 1510 & 418 & 300.1 & & \\
\hline $4: 14$ & $1: 38$ & 48 & 119 & 379 & 1510 & 421 & 302.2 & & \\
\hline $4: 16$ & $1: 40$ & 48 & 119 & 391 & 1503 & 428 & 307.2 & & \\
\hline $4: 18$ & $1: 42$ & 48 & 119 & 383 & 1509 & 419 & 300.8 & & \\
\hline $4: 20$ & $1: 44$ & 48 & 119 & 397 & 1503 & 402 & 288.6 & & \\
\hline $4: 22$ & $1: 46$ & 43 & 120 & 404 & 1512 & 431 & 309.4 & & \\
\hline $4: 24$ & $1: 48$ & 43 & 120 & 392 & 1505 & 428 & 307.2 & & \\
\hline $4: 26$ & $1: 50$ & 42 & 121 & 395 & 1504 & 418 & 300.1 & & \\
\hline $4: 28$ & $1: 52$ & 48 & 120 & 392 & 1500 & 436 & 313.0 & & \\
\hline $4: 30$ & $1: 54$ & 43 & 120 & 383 & 1503 & 464 & 333.1 & & \\
\hline $4: 32$ & $1: 56$ & 46 & 121 & 383 & 1514 & 421 & 302.2 & & \\
\hline $4: 34$ & $1: 58$ & 44 & 120 & 389 & 1507 & 393 & 282.1 & & \\
\hline $4: 36$ & $2: 00$ & 51 & 118 & 394 & 1510 & 431 & 309.4 & & \\
\hline $4: 38$ & $2: 02$ & 53 & 118 & 385 & 1515 & 394 & 282.8 & & \\
\hline $4: 40$ & $2: 04$ & 43 & 119 & 406 & 1510 & 428 & 307.2 & & \\
\hline $4: 42$ & $2: 06$ & 42 & 119 & 388 & 1504 & 405 & 290.7 & & \\
\hline $4: 44$ & $2: 08$ & 42 & 119 & 387 & 1510 & 430 & 308.7 & & \\
\hline $4: 46$ & $2: 10$ & 42 & 120 & 399 & 1507 & 424 & 304.4 & & \\
\hline $4: 48$ & $2: 12$ & 45 & 120 & 398 & 1502 & 421 & 302.2 & & \\
\hline $4: 50$ & $2: 14$ & 53 & 121 & 402 & 1501 & 413 & 296.5 & & \\
\hline $4: 52$ & $2: 16$ & 45 & 120 & 401 & 1511 & 401 & 287.8 & & \\
\hline $4: 54$ & $2: 18$ & 49 & 121 & 400 & 1508 & 421 & 302.2 & & \\
\hline $4: 56$ & $2: 20$ & 51 & 121 & 385 & 1503 & 419 & 300.8 & & \\
\hline $4: 58$ & $2: 22$ & 53 & 120 & 385 & 1502 & 412 & 295.7 & & \\
\hline $5: 00$ & $2: 24$ & 50 & 118 & 378 & 1504 & 363 & 260.6 & & \\
\hline $5: 02$ & $2: 26$ & 46 & 118 & 399 & 1504 & 382 & 274.2 & & \\
\hline $5: 04$ & $2: 28$ & 43 & 119 & 393 & 1506 & 384 & 275.6 & & \\
\hline $5: 06$ & $2: 30$ & 48 & 118 & 392 & 1505 & 426 & 305.8 & & \\
\hline $5: 08$ & $2: 32$ & 43 & 119 & 391 & 1508 & 401 & 287.8 & & \\
\hline $5: 10$ & $2: 34$ & 45 & 119 & 391 & 1503 & 402 & 288.6 & & \\
\hline $5: 12$ & $2: 36$ & 48 & 119 & 373 & 1518 & 417 & 299.3 & & \\
\hline $5: 14$ & $2: 38$ & 49 & 120 & 370 & 1506 & 434 & 311.5 & & \\
\hline $5: 16$ & $2: 40$ & 44 & 120 & 387 & 1508 & 421 & 302.2 & & \\
\hline $5: 18$ & $2: 42$ & 54 & 120 & 401 & 1510 & 411 & 295.0 & & \\
\hline $5: 20$ & $2: 44$ & 50 & 120 & 386 & 1508 & 429 & 307.9 & & \\
\hline $5: 22$ & $2: 46$ & 48 & 121 & 388 & 1509 & 421 & 302.2 & & \\
\hline $5: 24$ & $2: 48$ & 46 & 121 & 375 & 1511 & 405 & 290.7 & & \\
\hline $5: 26$ & $2: 50$ & 48 & 119 & 380 & 1500 & 384 & 275.6 & & \\
\hline $5: 28$ & $2: 52$ & 43 & 118 & 374 & 1505 & 427 & 306.5 & & \\
\hline $5: 30$ & $2: 54$ & 46 & 119 & 394 & 1518 & 408 & 292.9 & & \\
\hline $5: 32$ & $2: 56$ & 48 & 119 & 386 & 1512 & 418 & 300.1 & & \\
\hline $5: 34$ & $2: 59$ & 51 & 119 & 393 & 1510 & 420 & 301.5 & & \\
\hline $5: 36$ & 3:01 & 52 & 120 & 377 & 1502 & 420 & 301.5 & & \\
\hline $5: 38$ & $3: 03$ & 51 & 120 & 373 & 1504 & 424 & 304.4 & & \\
\hline $5: 40$ & $3: 05$ & 46 & 120 & 395 & 1509 & 428 & 307.2 & & \\
\hline $5: 42$ & $3: 07$ & 46 & 121 & 375 & 1513 & 424 & 304.4 & & \\
\hline $5: 44$ & $3: 09$ & 42 & 121 & 376 & 1507 & 421 & 302.2 & & \\
\hline
\end{tabular}

Page 2 
MATR_24.XLS

\begin{tabular}{|c|c|c|c|c|c|c|c|c|}
\hline $\begin{array}{c}\text { TIME OF } \\
\text { DAY }\end{array}$ & $\begin{array}{c}\text { ELAPSED } \\
\text { TIME }\end{array}$ & $\begin{array}{c}\text { FEED } \\
\text { PRESSURE }\end{array}$ & FEED TEMP & FEED FLOW & $\begin{array}{l}\text { ROTOR } \\
\text { SPEED }\end{array}$ & $\begin{array}{l}\text { PERMEATE } \\
\text { FLOW }\end{array}$ & $\begin{array}{l}\text { PERMEATE } \\
\text { FLUX }\end{array}$ & \\
\hline $5: 46$ & $3: 11$ & 49 & 121 & 385 & 1506 & 428 & 307.2 & \\
\hline $5: 48$ & $3: 13$ & 45 & 120 & 381 & 1512 & 388 & 278.5 & \\
\hline $5: 50$ & $3: 15$ & 42 & 118 & 390 & 1506 & 412 & 295.7 & \\
\hline $5: 52$ & $3: 17$ & 52 & 118 & 387 & 1508 & 381 & 273.5 & \\
\hline $5: 54$ & $3: 19$ & 52 & 119 & 392 & 1499 & 415 & 297.9 & End Steady-state \\
\hline \multicolumn{2}{|c|}{ Steady-state Avg. } & 47 & 120 & 388 & 1507 & 416 & 299 & \\
\hline \multicolumn{2}{|c|}{ Maximum } & 54 & 121 & 406 & 1518 & 464 & 333 & \\
\hline Minimum & & 42 & 118 & 370 & 1499 & 363 & 261 & \\
\hline \multicolumn{2}{|c|}{ Standard Deviation } & 3.5 & 1.0 & 8.3 & 4.6 & 17.4 & 12.5 & \\
\hline \multirow{2}{*}{\multicolumn{4}{|c|}{ TEST CONDITIONS: MATR_24.XLS }} & & & & & \\
\hline & & & & Temp. $=$ & $120^{\circ} \mathrm{F}$ & & & \\
\hline & & & & Press. $=$ & $45 \mathrm{psig}$ & & & \\
\hline \multicolumn{3}{|c|}{ Date Run: 18-May-96 } & & Rotor $=$ & $1500 \mathrm{rpm}$ & & & \\
\hline & & & & Solids $=$ & $15 w t \%$ & & & \\
\hline & & & & Flow $=2$ & $400 \mathrm{~L} / \mathrm{hr}$ & & & \\
\hline
\end{tabular}




\section{SpinTek II High Shear Rotary Membrane System}

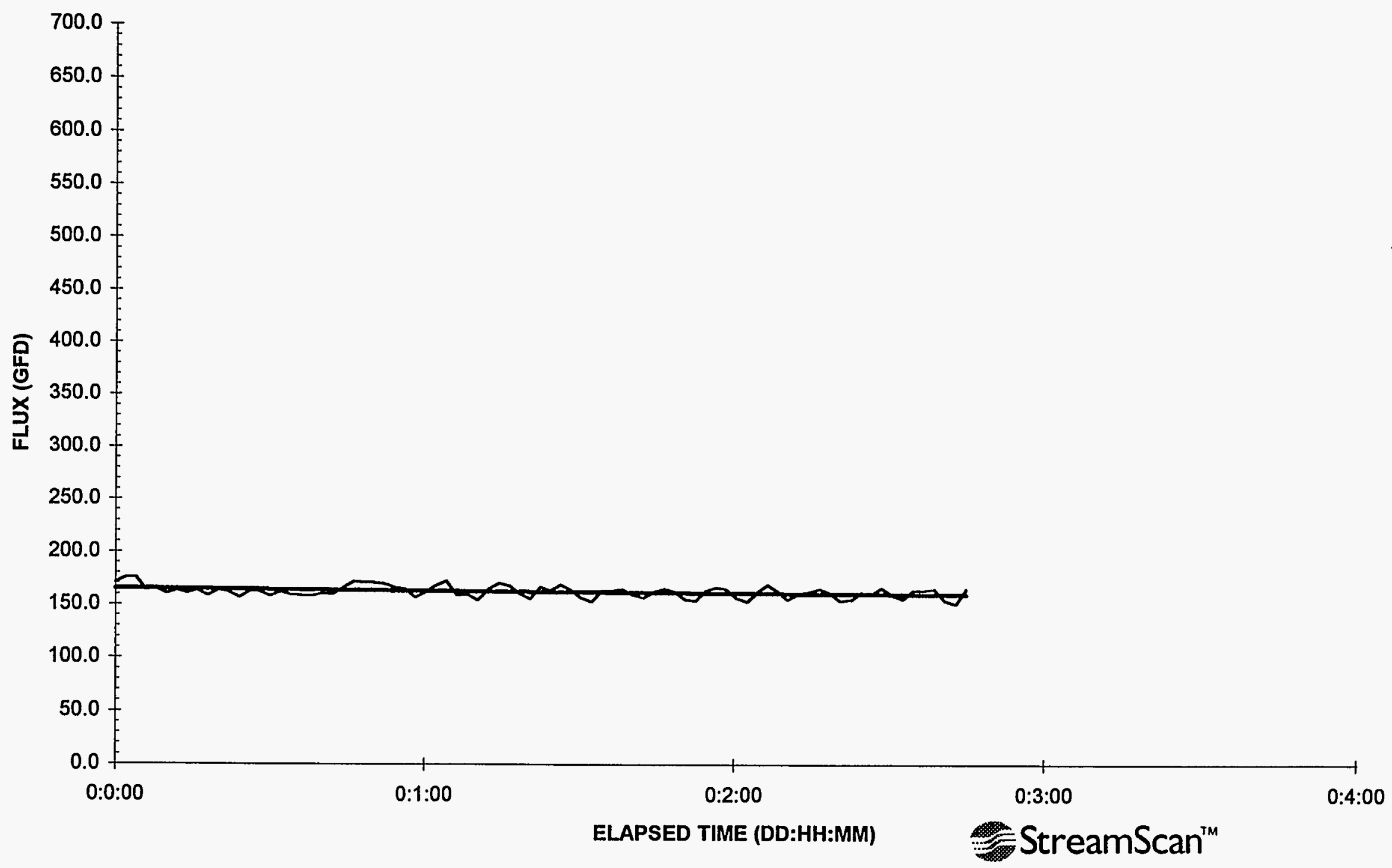




\begin{tabular}{|c|c|c|c|c|c|c|c|c|}
\hline $\begin{array}{c}\text { TIME OF } \\
\text { DAY }\end{array}$ & $\begin{array}{c}\text { ELAPSED } \\
\text { TIME }\end{array}$ & $\begin{array}{c}\text { FEED } \\
\text { PRESSURE }\end{array}$ & FEED TEMP & FEED FLOW & $\begin{array}{l}\text { ROTOR } \\
\text { SPEED }\end{array}$ & $\begin{array}{c}\text { PERMEATE } \\
\text { FLOW }\end{array}$ & $\begin{array}{c}\text { PERMEATE } \\
\text { FLUX }\end{array}$ & \\
\hline $7: 48$ & $0: 00$ & 80 & 58 & 146 & 1503 & 238 & 170.8 & \\
\hline $7: 50$ & $0: 02$ & 80 & 59 & 147 & 1505 & 244 & 175.2 & \\
\hline $7: 52$ & $0: 04$ & 65 & 60 & 146 & 1505 & 244 & 175.2 & \\
\hline $7: 54$ & $0: 06$ & 66 & 61 & 145 & 1510 & 228 & 163.7 & \\
\hline $7: 56$ & $0: 08$ & 73 & 62 & 146 & 1495 & 230 & 165.1 & \\
\hline $7: 58$ & $0: 10$ & 77 & 62 & 147 & 1498 & 223 & 160.1 & \\
\hline $8: 00$ & $0: 12$ & 75 & 62 & 147 & 1497 & 227 & 162.9 & \\
\hline $8: 02$ & $0: 14$ & 79 & 62 & 146 & 1505 & 224 & 160.8 & \\
\hline $8: 04$ & $0: 16$ & 71 & 62 & 146 & 1503 & 227 & 162.9 & \\
\hline $8: 06$ & $0: 18$ & 72 & 62 & 147 & 1507 & 221 & 158.6 & \\
\hline $8: 08$ & $0: 20$ & 71 & 62 & 146 & 1494 & 228 & 163.7 & \\
\hline $8: 10$ & $0: 22$ & 69 & 62 & 147 & 1502 & 225 & 161.5 & \\
\hline $8: 12$ & $0: 24$ & 74 & 61 & 147 & 1500 & 218 & 156.5 & \\
\hline $8: 14$ & $0: 26$ & 76 & 61 & 147 & 1504 & 226 & 162.2 & \\
\hline $8: 16$ & $0: 28$ & 70 & 61 & 146 & 1496 & 226 & 162.2 & \\
\hline $8: 18$ & $0: 30$ & 66 & 61 & 146 & 1502 & 220 & 157.9 & \\
\hline $8: 20$ & $0: 32$ & 82 & 61 & 146 & 1503 & 225 & 161.5 & \\
\hline $8: 22$ & $0: 34$ & 72 & 61 & 146 & 1502 & 222 & 159.4 & \\
\hline $8: 24$ & $0: 36$ & 77 & 61 & 146 & 1500 & 221 & 158.6 & \\
\hline $8: 26$ & $0: 38$ & 82 & 61 & 144 & 1503 & 220 & 157.9 & \\
\hline $8: 28$ & $0: 40$ & 76 & 61 & 144 & 1503 & 223 & 160.1 & \\
\hline $8: 30$ & $0: 42$ & 81 & 60 & 143 & 1508 & 222 & 159.4 & \\
\hline $8: 32$ & $0: 44$ & 76 & 59 & 144 & 1505 & 230 & 165.1 & Begin Steady-state \\
\hline $8: 35$ & $0: 46$ & 75 & 59 & 143 & 1504 & 239 & 171.6 & \\
\hline $8: 37$ & $0: 48$ & 68 & 59 & 143 & 1503 & 238 & 170.8 & \\
\hline $8: 39$ & $0: 50$ & 81 & 59 & 144 & 1499 & 238 & 170.8 & \\
\hline $8: 41$ & $0: 52$ & 75 & 60 & 143 & 1500 & 236 & 169.4 & \\
\hline $8: 43$ & $0: 54$ & 72 & 61 & 142 & 1511 & 231 & 165.8 & \\
\hline $8: 45$ & $0: 56$ & 73 & 60 & 143 & 1495 & 229 & 164.4 & \\
\hline $8: 47$ & $0: 58$ & 72 & 60 & 142 & 1503 & 218 & 156.5 & \\
\hline $8: 49$ & $1: 00$ & 69 & 59 & 143 & 1504 & 225 & 161.5 & \\
\hline $8: 51$ & $1: 02$ & 81 & 60 & 143 & 1505 & 234 & 168.0 & \\
\hline $8: 53$ & $1: 04$ & 69 & 60 & 143 & 1502 & 240 & 172.3 & \\
\hline $8: 55$ & $1: 06$ & 68 & 60 & 143 & 1503 & 221 & 158.6 & \\
\hline $8: 57$ & $1: 08$ & 68 & 60 & 142 & 1500 & 222 & 159.4 & \\
\hline $8: 59$ & $1: 10$ & 68 & 60 & 142 & 1503 & 215 & 154.3 & \\
\hline $9: 01$ & $1: 12$ & 68 & 60 & 143 & 1495 & 229 & 164.4 & \\
\hline $9: 03$ & $1: 14$ & 80 & 60 & 143 & 1503 & 237 & 170.1 & \\
\hline $9: 05$ & $1: 16$ & 67 & 60 & 142 & 1502 & 234 & 168.0 & \\
\hline $9: 07$ & $1: 18$ & 65 & 60 & 141 & 1503 & 223 & 160.1 & \\
\hline $9: 09$ & $1: 20$ & 64 & 60 & 142 & 1505 & 217 & 155.8 & \\
\hline $9: 11$ & $1: 22$ & 68 & 60 & 143 & 1500 & 232 & 166.5 & \\
\hline $9: 13$ & $1: 24$ & 75 & 60 & 143 & 1507 & 227 & 162.9 & \\
\hline $9: 15$ & $1: 26$ & 69 & 60 & 144 & 1503 & 235 & 168.7 & \\
\hline $9: 17$ & $1: 28$ & 74 & 60 & 141 & 1508 & 228 & 163.7 & \\
\hline
\end{tabular}


MATR_25.XLS

\begin{tabular}{|c|c|c|c|c|c|c|c|c|}
\hline $\begin{array}{l}\text { TIME OF } \\
\text { DAY }\end{array}$ & $\begin{array}{c}\text { ELAPSED } \\
\text { TIME }\end{array}$ & \begin{tabular}{|c|} 
FEED \\
PRESSURE \\
\end{tabular} & FEED TEMP & FEED FLOW & \begin{tabular}{|c|} 
ROTOR \\
SPEED \\
\end{tabular} & $\begin{array}{c}\text { PERMEATE } \\
\text { FLOW }\end{array}$ & \begin{tabular}{|c|}
$\begin{array}{c}\text { PERMEATE } \\
\text { FLUX }\end{array}$ \\
\end{tabular} & \\
\hline $9: 19$ & $1: 30$ & 67 & 60 & 141 & 1503 & 218 & 156.5 & \\
\hline $9: 21$ & $1: 32$ & 66 & 60 & 141 & 1503 & 213 & 152.9 & \\
\hline $9: \overline{23}$ & $1: 34$ & 79 & 60 & 141 & 1495 & 227 & 162.9 & \\
\hline $9: 25$ & $1: 36$ & 67 & 60 & 141 & 1507 & 227 & 162.9 & \\
\hline $9: 27$ & $1: 38$ & 74 & 60 & 142 & 1492 & 229 & 164.4 & \\
\hline $9: 29$ & $1: 40$ & 74 & 60 & 142 & 1501 & 222 & 159.4 & \\
\hline $9: 31$ & $1: 42$ & 68 & 60 & 141 & 1503 & 218 & 156.5 & \\
\hline $9: 33$ & $1: 44$ & 77 & 60 & 142 & 1503 & 226 & 162.2 & \\
\hline $9: 35$ & $1: 46$ & 75 & 60 & 142 & 1497 & 230 & 165.1 & \\
\hline $9: 37$ & $1: 48$ & 78 & 60 & 141 & 1505 & 226 & 162.2 & \\
\hline $9: 39$ & $1: 50$ & 67 & 60 & 141 & 1504 & 216 & 155.1 & \\
\hline $9: 41$ & $1: 52$ & 74 & 60 & 140 & 1493 & 214 & 153.6 & \\
\hline $9: 43$ & $1: 54$ & 72 & 59 & 142 & 1500 & 227 & 162.9 & \\
\hline $9: 45$ & $1: 56$ & 70 & 60 & 141 & 1506 & 232 & 166.5 & \\
\hline $9: 47$ & $1: 58$ & $\overline{73}$ & 60 & 142 & 1493 & 229 & 164.4 & \\
\hline $9: 49$ & $2: 00$ & 75 & 60 & 137 & 1495 & 218 & 156.5 & \\
\hline $9: 51$ & $2: 02$ & 65 & 60 & 136 & 1503 & 213 & 152.9 & \\
\hline $9: 53$ & $2: 04$ & 74 & 60 & 136 & 1503 & 226 & 162.2 & \\
\hline $9: 55$ & $2: 06$ & 72 & 60 & 136 & 1507 & 235 & 168.7 & \\
\hline $9: 57$ & $2: 08$ & 71 & 60 & 136 & 1499 & 227 & 162.9 & \\
\hline $9: 59$ & $2: 10$ & 74 & 60 & 137 & 1503 & 216 & 155.1 & \\
\hline $10: 01$ & $2: 12$ & 66 & 60 & 135 & 1508 & 224 & 160.8 & \\
\hline $10: 03$ & $2: 14$ & 74 & 60 & 135 & 1499 & 226 & 162.2 & \\
\hline $10: 05$ & $2: 16$ & 70 & 60 & 136 & 1493 & 230 & 165.1 & \\
\hline $10: 07$ & $2: 18$ & 64 & 61 & 135 & 1503 & 224 & 160.8 & \\
\hline $10: 09$ & $2: 20$ & 70 & 61 & 136 & 1504 & 214 & 153.6 & \\
\hline $10: 11$ & $2: 22$ & 74 & 60 & 135 & 1503 & 216 & 155.1 & \\
\hline $10: 13$ & $2: 24$ & 67 & 60 & 135 & 1497 & 225 & 161.5 & \\
\hline $10: 15$ & $2: 26$ & 77 & 60 & 136 & 1501 & 223 & 160.1 & \\
\hline $10: 17$ & $2: 28$ & 69 & 60 & 136 & 1503 & 231 & 165.8 & \\
\hline $10: 19$ & $2: 30$ & 64 & 61 & 135 & 1497 & 222 & 159.4 & \\
\hline $10: 21$ & $2: 32$ & 66 & 60 & 135 & 1498 & 217 & 155.8 & \\
\hline $10: 23$ & $2: 34$ & 71 & 60 & 136 & 1507 & 228 & 163.7 & \\
\hline $10: 25$ & $2: 36$ & 73 & 60 & 136 & 1497 & 228 & 163.7 & \\
\hline $10: 27$ & $2: 38$ & 79 & 60 & 136 & 1494 & 230 & 165.1 & \\
\hline $10: 29$ & $2: 41$ & 65 & 60 & 135 & 1501 & 215 & 154.3 & \\
\hline $10: 31$ & $2: 43$ & 79 & 60 & 135 & 1499 & 210 & 150.7 & \\
\hline $10: 33$ & $2: 45$ & 75 & 59 & 137 & 1504 & 230 & 165.1 & End Steady-state \\
\hline \multirow{2}{*}{\multicolumn{2}{|c|}{ Steady-state Avg. }} & & & & & 20 & 109 & \\
\hline & & 71 & 60 & 140 & 1501 & 226 & 162 & \\
\hline \multicolumn{2}{|l|}{ Maximum } & 81 & 61 & 144 & 1511 & 240 & 172 & \\
\hline Minimum & & 64 & 59 & 135 & 1492 & 210 & 151 & \\
\hline \multicolumn{2}{|c|}{ Standard Deviation } & 4.6 & 0.4 & 3.3 & 4.3 & 7.5 & 5.4 & \\
\hline \multirow{2}{*}{\multicolumn{4}{|c|}{ TEST CONDITIONS: MATR_25.XLS }} & & & & & \\
\hline & & & & Temp. $=$ & $60 \mathrm{~F}$ & & & \\
\hline & & & & Press. $=$ & 75 psig & & & \\
\hline \multicolumn{3}{|c|}{ Date Run: 18-May-96 } & & Rotor $=$ & $1500 \mathrm{rpm}$ & & & \\
\hline & & & & Solids $=$ & $15 w t \%$ & & & \\
\hline & & & & Flow $=$ & $400 \mathrm{~L} / \mathrm{hr}$ & & & \\
\hline
\end{tabular}


SpinTek Il High Shear Rotary Membrane System

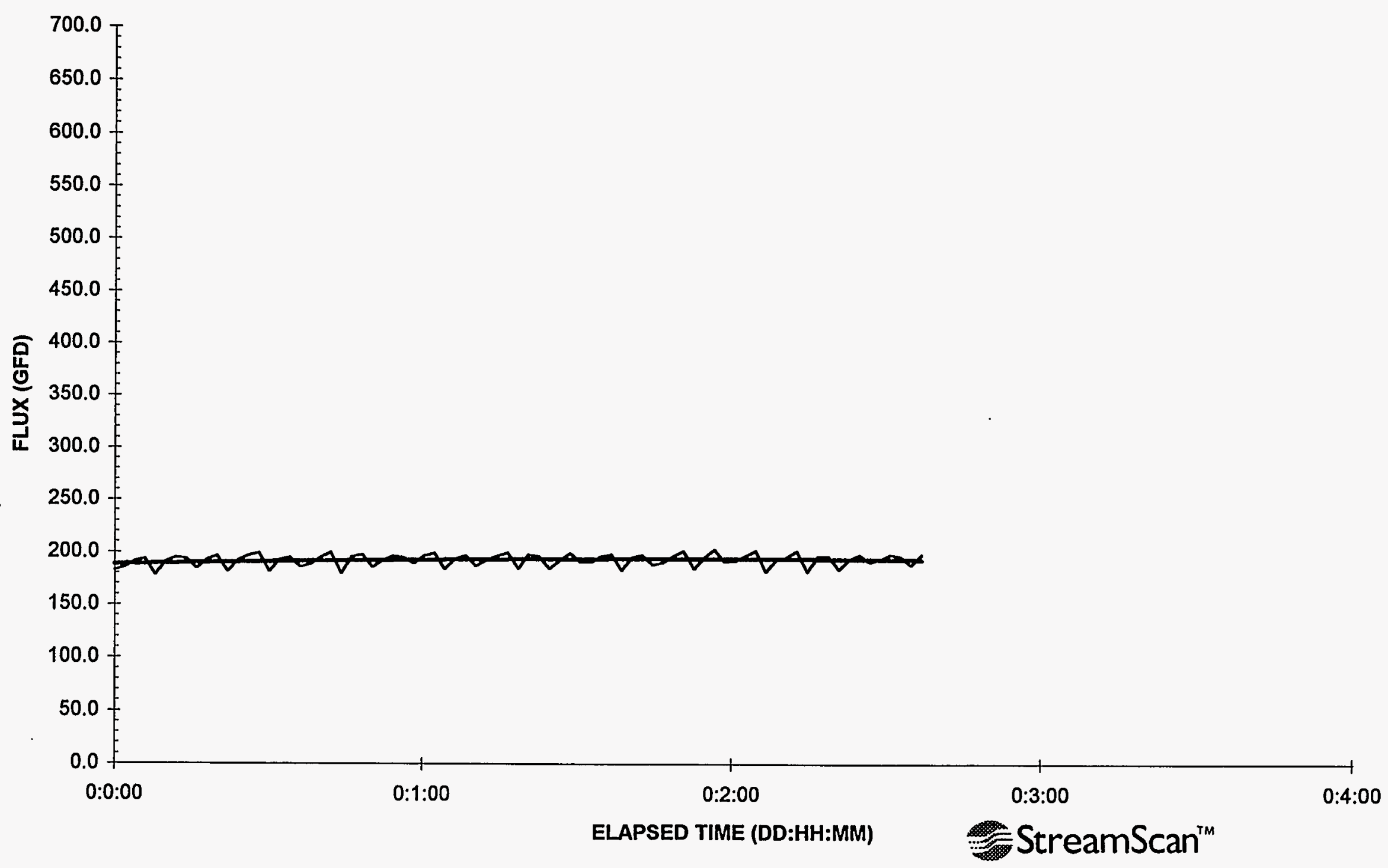




\begin{tabular}{|c|c|c|c|c|c|c|c|c|}
\hline $\begin{array}{c}\text { TIME OF } \\
\text { DAY }\end{array}$ & $\begin{array}{l}\text { ELAPSED } \\
\text { TIME }\end{array}$ & $\begin{array}{c}\text { FEED } \\
\text { PRESSURE }\end{array}$ & FEED TEMP & FEED FLOW & $\begin{array}{l}\text { ROTOR } \\
\text { SPEED }\end{array}$ & $\begin{array}{l}\text { PERMEATE } \\
\text { FLOW }\end{array}$ & $\begin{array}{l}\text { PERMEATE } \\
\text { FLUX }\end{array}$ & \\
\hline $11: 57$ & $0: 00$ & 46 & 91 & 402 & 1491 & 254 & 182.3 & \\
\hline $11: 59$ & $0: 01$ & 52 & 89 & 392 & 1491 & 258 & 185.2 & \\
\hline $12: 01$ & $0: 03$ & 54 & 89 & 391 & 1483 & 266 & 190.9 & \\
\hline $12: 03$ & $0: 05$ & 52 & 90 & 395 & 1490 & 269 & 193.1 & \\
\hline $12: 05$ & $0: 07$ & 45 & 90 & 390 & 1489 & 248 & 178.0 & \\
\hline $12: 07$ & $0: 10$ & 48 & 89 & 388 & 1491 & 265 & 190.2 & \\
\hline $12: 09$ & $0: 12$ & 49 & 90 & 393 & 1489 & 271 & 194.5 & \\
\hline $12: 11$ & $0: 14$ & 48 & 90 & 395 & 1485 & 269 & 193.1 & \\
\hline $12: 13$ & $0: 16$ & 48 & 89 & 392 & 1490 & 257 & 184.5 & \\
\hline $12: 15$ & $0: 18$ & 45 & 89 & 391 & 1491 & 268 & 192.4 & \\
\hline $12: 17$ & $0: 20$ & 40 & 90 & 391 & 1492 & 273 & 196.0 & \\
\hline $12: 19$ & $0: 22$ & 47 & 91 & 399 & 1489 & 253 & 181.6 & \\
\hline $12: 21$ & $0: 24$ & 42 & 89 & 392 & 1487 & 267 & 191.7 & \\
\hline $12: 23$ & $0: 26$ & 49 & 89 & 395 & 1485 & 274 & 196.7 & \\
\hline $12: 25$ & $0: 28$ & 40 & 90 & 401 & 1485 & 277 & 198.8 & \\
\hline $12: 27$ & $0: 30$ & 41 & 90 & 392 & 1485 & 253 & 181.6 & Begin Steady-state \\
\hline $12: 29$ & $0: 32$ & 54 & 88 & 395 & 1492 & 268 & 192.4 & \\
\hline $12: 31$ & $0: 34$ & 45 & 89 & 391 & 1485 & 271 & 194.5 & \\
\hline $12: 33$ & $0: 36$ & 40 & 91 & 391 & 1489 & 259 & 185.9 & \\
\hline $12: 35$ & $0: 38$ & 45 & 89 & 393 & 1490 & 262 & 188.1 & \\
\hline $12: 37$ & $0: 40$ & 44 & 89 & 392 & 1487 & 271 & 194.5 & \\
\hline $12: 39$ & $0: 42$ & 44 & 90 & 396 & 1490 & 278 & 199.6 & \\
\hline $12: 41$ & $0: 44$ & 41 & 90 & 392 & 1492 & 250 & 179.5 & \\
\hline $12: 43$ & $0: 46$ & 43 & 89 & 394 & 1489 & 272 & 195.2 & \\
\hline $12: 45$ & $0: 48$ & 41 & 90 & 391 & 1485 & 275 & 197.4 & \\
\hline $12: 47$ & $0: 50$ & 43 & 91 & 397 & 1482 & 259 & 185.9 & \\
\hline $12: 49$ & $0: 52$ & 40 & 89 & 397 & 1489 & 267 & 191.7 & \\
\hline $12: 51$ & $0: 54$ & 47 & 89 & 396 & 1489 & 273 & 196.0 & \\
\hline $12: 53$ & $0: 56$ & 47 & 91 & 396 & 1488 & 271 & 194.5 & \\
\hline $12: 55$ & $0: 58$ & 43 & 89 & 395 & 1483 & 263 & 188.8 & \\
\hline $12: 57$ & $1: 00$ & 49 & 89 & 392 & 1488 & 273 & 196.0 & \\
\hline $12: 59$ & $1: 02$ & 44 & 90 & 395 & 1491 & 277 & 198.8 & \\
\hline $13: 01$ & $1: 04$ & 44 & 90 & 392 & 1490 & 256 & 183.8 & \\
\hline $13: 03$ & $1: 06$ & 51 & 88 & 395 & 1493 & 269 & 193.1 & \\
\hline $13: 05$ & $1: 08$ & 53 & 89 & 400 & 1491 & 274 & 196.7 & \\
\hline $13: 07$ & $1: 10$ & 52 & 90 & 402 & 1490 & 260 & 186.6 & \\
\hline $13: 09$ & $1: 12$ & 41 & 89 & 392 & 1495 & 268 & 192.4 & \\
\hline $13: 11$ & $1: 14$ & 48 & 89 & 392 & 1489 & 273 & 196.0 & \\
\hline $13: 13$ & $1: 16$ & 40 & 90 & 395 & 1489 & 278 & 199.6 & \\
\hline $13: 15$ & $1: 18$ & 51 & 89 & 404 & 1489 & 257 & 184.5 & \\
\hline $13: 17$ & $1: 20$ & 48 & 88 & 394 & 1482 & 275 & 197.4 & \\
\hline $13: 19$ & $1: 22$ & 43 & 90 & 392 & 1482 & 273 & 196.0 & \\
\hline $13: 21$ & $1: 24$ & 45 & 90 & 390 & 1485 & 257 & 184.5 & \\
\hline $13: 23$ & $1: 26$ & 45 & 88 & 392 & 1490 & 268 & 192.4 & \\
\hline $13: 25$ & $1: 28$ & 50 & 89 & 394 & 1490 & 277 & 198.8 & \\
\hline
\end{tabular}


MATR_26.XLS

\begin{tabular}{|c|c|c|c|c|c|c|c|c|}
\hline $\begin{array}{l}\text { TIME OF } \\
\text { DAY }\end{array}$ & $\begin{array}{c}\text { ELAPSED } \\
\text { TIME }\end{array}$ & $\begin{array}{c}\text { FEED } \\
\text { PRESSURE } \\
\end{array}$ & FEED TEMP & FEED FLOW & $\begin{array}{l}\text { ROTOR } \\
\text { SPEED }\end{array}$ & \begin{tabular}{|c|} 
PERMEATE \\
FLOW
\end{tabular} & \begin{tabular}{|c|} 
PERMEATE \\
FLUX
\end{tabular} & \\
\hline 13:27 & $1: 30$ & 44 & 91 & 392 & 1489 & 265 & 190.2 & \\
\hline $13: 29$ & $1: 32$ & 46 & 89 & 393 & 1485 & 265 & 190.2 & \\
\hline $13: 31$ & $1: 34$ & 51 & 89 & 389 & 1488 & 273 & 196.0 & \\
\hline $13: 33$ & $1: 36$ & 40 & 90 & 389 & 1489 & 276 & 198.1 & \\
\hline $13: 35$ & $1: 38$ & 41 & 90 & 394 & 1477 & 255 & 183.0 & \\
\hline $13: 37$ & $1: 40$ & 47 & 88 & 392 & 1491 & 272 & 195.2 & \\
\hline $13: 39$ & $1: 42$ & 41 & 89 & 391 & 1489 & 275 & 197.4 & \\
\hline $13: 42$ & $1: 44$ & 54 & 90 & 394 & 1487 & 262 & 188.1 & \\
\hline $13: 44$ & $1: 46$ & 45 & 89 & 394 & 1486 & 265 & 190.2 & \\
\hline $13: 46$ & $1: 48$ & 47 & 89 & 389 & 1493 & 273 & 196.0 & \\
\hline $13: 48$ & $1: 50$ & 48 & 90 & 392 & 1481 & 280 & 201.0 & \\
\hline $13: 50$ & $1: 52$ & 41 & 90 & 396 & 1483 & 257 & 184.5 & \\
\hline $13: 52$ & $1: 54$ & 40 & 88 & 392 & 1487 & 272 & 195.2 & \\
\hline $13: 54$ & $1: 56$ & 46 & 89 & 393 & 1489 & 282 & 202.4 & \\
\hline $13: 56$ & $1: 58$ & 47 & 90 & 392 & 1492 & 266 & 190.9 & \\
\hline $13: 58$ & $2: 00$ & 40 & 89 & 397 & 1489 & 267 & 191.7 & \\
\hline $14: 00$ & $2: 02$ & 41 & 89 & 388 & 1486 & 274 & 196.7 & \\
\hline $14: 02$ & $2: 04$ & 41 & 90 & 397 & 1487 & 281 & 201.7 & \\
\hline $14: 04$ & $2: 06$ & 43 & 90 & 391 & 1487 & 253 & 181.6 & \\
\hline $14: 06$ & $2: 08$ & 48 & 88 & 388 & 1489 & 268 & 192.4 & \\
\hline $14: 08$ & $2: 10$ & 40 & 89 & 396 & 1489 & 272 & 195.2 & \\
\hline $14: 10$ & $2: 12$ & 48 & 90 & 392 & 1482 & 280 & 201.0 & \\
\hline $14: 12$ & $2: 14$ & 54 & 89 & 389 & 1479 & 253 & 181.6 & \\
\hline $14: 14$ & $2: 16$ & 49 & 89 & 397 & 1486 & 273 & 196.0 & \\
\hline $14: 16$ & $2: 19$ & 53 & 90 & 392 & 1475 & 273 & 196.0 & \\
\hline $14: 18$ & $2: 21$ & 48 & 90 & 389 & 1489 & 256 & 183.8 & \\
\hline $14: 20$ & $2: 23$ & 44 & 88 & 391 & 1487 & 269 & 193.1 & \\
\hline $14: 22$ & $2: 25$ & 54 & 89 & 398 & 1487 & 275 & 197.4 & \\
\hline $14: 24$ & $2: 27$ & 44 & 91 & 388 & 1481 & 266 & 190.9 & \\
\hline $14: 26$ & $2: 29$ & 53 & 89 & 395 & 1487 & 269 & 193.1 & \\
\hline $14: 28$ & $2: 31$ & 42 & 90 & 385 & 1490 & 276 & 198.1 & \\
\hline $14: 30$ & $2: 33$ & 41 & 91 & 399 & 1484 & 273 & 196.0 & \\
\hline $14: 32$ & $2: 35$ & 45 & 89 & 393 & 1486 & 262 & 188.1 & \\
\hline $14: 34$ & $2: 37$ & 43 & 89 & 392 & 1486 & 276 & 198.1 & End Steady-state \\
\hline \multicolumn{2}{|c|}{ Steady-state Avg. } & 45 & 89 & 393 & 1487 & 268 & 193 & \\
\hline \multicolumn{2}{|c|}{ Maximum } & 54 & 91 & 404 & 1495 & 282 & 202 & \\
\hline Minimum & & 40 & 88 & 385 & 1475 & 250 & 179 & \\
\hline \multicolumn{2}{|c|}{ Standard Deviation } & 4.3 & 0.8 & 3.4 & 3.8 & 8.0 & 5.7 & \\
\hline \multicolumn{4}{|c|}{ TEST CONDITIONS: MATR_26.XLS } & Temp. $=$ & $90 \mathrm{~F}$ & & & \\
\hline & & & & Press. $=$ & $45 \mathrm{psig}$ & & & \\
\hline \multicolumn{3}{|c|}{ Date Run: 18-May-96 } & & Rotor $=$ & $1500 \mathrm{rpm}$ & & & \\
\hline & & & & Solids = & $1 w t \%$ & & & \\
\hline & & & & Flow $=$ & $400 \mathrm{~L} / \mathrm{hr}$ & & & \\
\hline
\end{tabular}


SpinTek II High Shear Rotary Membrane System

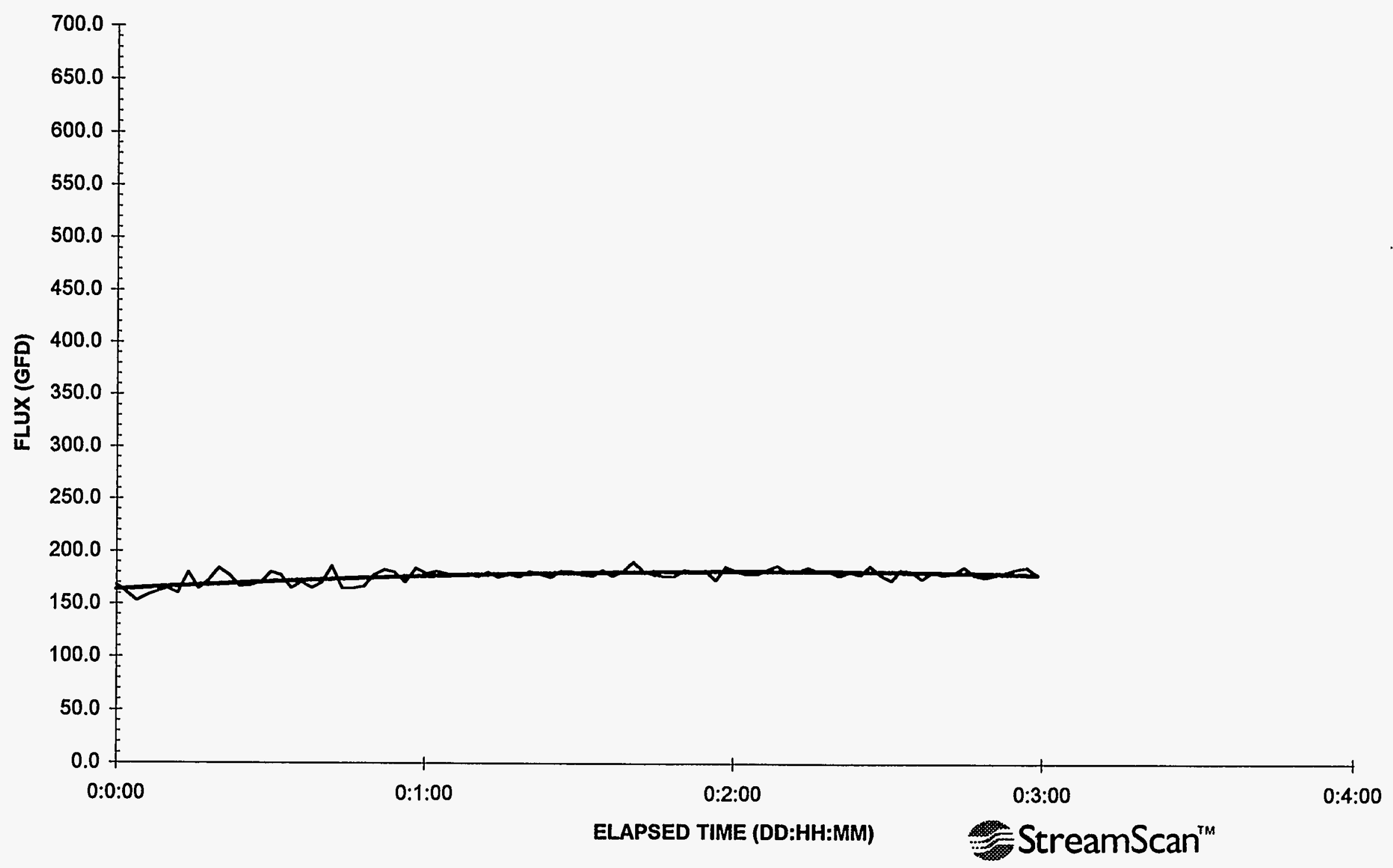




\begin{tabular}{|c|c|c|c|c|c|c|c|c|}
\hline $\begin{array}{c}\text { TIME OF } \\
\text { DAY }\end{array}$ & $\begin{array}{c}\text { ELAPSED } \\
\text { TIME }\end{array}$ & $\begin{array}{c}\text { FEED } \\
\text { PRESSURE }\end{array}$ & FEED TEMP & FEED FLOW & $\begin{array}{l}\text { ROTOR } \\
\text { SPEED }\end{array}$ & $\begin{array}{c}\text { PERMEATE } \\
\text { FLOW }\end{array}$ & $\begin{array}{c}\text { PERMEATE } \\
\text { FLUX }\end{array}$ & \\
\hline $15: 13$ & $0: 00$ & 58 & 60 & 225 & 2008 & 234 & 168.0 & \\
\hline $15: 15$ & $0: 01$ & 67 & 61 & 234 & 2009 & 224 & 160.8 & \\
\hline $15: 17$ & $0: 03$ & 65 & 61 & 230 & 2005 & 213 & 152.9 & \\
\hline $15: 19$ & $0: 05$ & 53 & 61 & 231 & 2008 & 220 & 157.9 & \\
\hline $15: 21$ & $0: 07$ & 50 & 61 & 234 & 2009 & 225 & 161.5 & \\
\hline $15: 23$ & $0: 10$ & 50 & 61 & 230 & 2005 & 229 & 164.4 & \\
\hline $15: 25$ & $0: 12$ & 49 & 61 & 230 & 2007 & 223 & 160.1 & \\
\hline $15: 27$ & $0: 14$ & 53 & 61 & 213 & 2003 & 250 & 179.5 & \\
\hline $15: 29$ & $0: 16$ & 53 & 61 & 211 & 2012 & 229 & 164.4 & \\
\hline $15: 31$ & $0: 18$ & 53 & 60 & 213 & 2006 & 240 & 172.3 & \\
\hline $15: 33$ & $0: 20$ & 51 & 60 & 213 & 2001 & 256 & 183.8 & \\
\hline $15: 35$ & $0: 22$ & 67 & 60 & 213 & 2001 & 247 & 177.3 & \\
\hline $15: 37$ & $0: 24$ & 54 & 61 & 214 & 2008 & 232 & 166.5 & \\
\hline $15: 39$ & $0: 26$ & 56 & 61 & 213 & 2006 & 233 & 167.3 & \\
\hline $15: 41$ & $0: 28$ & 67 & 60 & 214 & 2001 & 237 & 170.1 & \\
\hline $15: 43$ & $0: 30$ & 55 & 61 & 213 & 2000 & 251 & 180.2 & \\
\hline $15: 45$ & $0: 32$ & 58 & 60 & 214 & 2006 & 247 & 177.3 & \\
\hline $15: 47$ & $0: 34$ & 54 & 60 & 214 & 2009 & 230 & 165.1 & \\
\hline $15: 49$ & $0: 36$ & 57 & 60 & 213 & 2000 & 238 & 170.8 & \\
\hline $15: 51$ & $0: 38$ & 61 & 60 & 212 & 2006 & 230 & 165.1 & \\
\hline $15: 53$ & $0: 40$ & 53 & 60 & 213 & 2001 & 237 & 170.1 & \\
\hline $15: 55$ & $0: 42$ & 53 & 60 & 213 & 2014 & 259 & 185.9 & \\
\hline $15: 57$ & $0: 44$ & 57 & 61 & 213 & 2002 & 230 & 165.1 & \\
\hline $15: 59$ & $0: 46$ & 55 & 61 & 214 & 2010 & 229 & 164.4 & \\
\hline $16: 01$ & $0: 48$ & 60 & 61 & 206 & 2016 & 232 & 166.5 & \\
\hline $16: 03$ & $0: 50$ & 69 & 61 & 203 & 2006 & 247 & 177.3 & \\
\hline $16: 05$ & $0: 52$ & 58 & 61 & 201 & 2008 & 254 & 182.3 & \\
\hline $16: 07$ & $0: 54$ & 54 & 60 & 202 & 2003 & 251 & 180.2 & \\
\hline $16: 09$ & $0: 56$ & 68 & 60 & 201 & 2004 & 238 & 170.8 & \\
\hline $16: 11$ & $0: 58$ & 54 & 60 & 200 & 2001 & 256 & 183.8 & \\
\hline $16: 13$ & $1: 00$ & 56 & 60 & 202 & 2009 & 249 & 178.7 & Begin Steady-state \\
\hline $16: 15$ & $1: 02$ & 60 & 60 & 201 & 2003 & 252 & 180.9 & \\
\hline $16: 17$ & $1: 04$ & 60 & 60 & 201 & 2002 & 249 & 178.7 & \\
\hline $16: 19$ & $1: 06$ & 55 & 60 & 202 & 2002 & 246 & 176.6 & \\
\hline $16: 21$ & 1:08 & 54 & 60 & 201 & 2002 & 249 & 178.7 & \\
\hline $16: 23$ & $1: 10$ & 60 & 60 & 201 & 2000 & 245 & 175.9 & \\
\hline $16: 25$ & $1: 12$ & 54 & 60 & 202 & 2002 & 251 & 180.2 & \\
\hline $16: 27$ & $1: 14$ & 65 & 60 & 203 & 2004 & 244 & 175.2 & \\
\hline $16: 29$ & $1: 16$ & 62 & 61 & 201 & 2001 & 248 & 178.0 & \\
\hline $16: 31$ & $1: 18$ & 52 & 60 & 200 & 1999 & 245 & 175.9 & \\
\hline $16: 33$ & $1: 20$ & 52 & 60 & 202 & 2006 & 252 & 180.9 & \\
\hline $16: 35$ & $1: 22$ & 54 & 61 & 201 & 2013 & 249 & 178.7 & \\
\hline $16: 37$ & $1: 24$ & 68 & 60 & 201 & 2002 & 244 & 175.2 & \\
\hline $16: 39$ & $1: 26$ & 60 & 60 & 202 & 2002 & 253 & 181.6 & \\
\hline $16: 41$ & $1: 28$ & 55 & 61 & 202 & 1999 & 253 & 181.6 & \\
\hline
\end{tabular}




\begin{tabular}{|c|c|c|c|c|c|c|c|c|}
\hline $\begin{array}{c}\text { TIME OF } \\
\text { DAY }\end{array}$ & $\begin{array}{c}\text { ELAPSED } \\
\text { TIME }\end{array}$ & \begin{tabular}{|c|} 
FEED \\
PRESSURE
\end{tabular} & FEED TEMP & FEED FLOW & $\begin{array}{l}\text { ROTOR } \\
\text { SPEED }\end{array}$ & $\begin{array}{c}\text { PERMEATE } \\
\text { FLOW }\end{array}$ & $\begin{array}{c}\text { PERMEATE } \\
\text { FLUX }\end{array}$ & \\
\hline $16: 44$ & $1: 30$ & 54 & 60 & 201 & 2013 & 248 & 178.0 & \\
\hline $16: 46$ & $1: 32$ & 57 & 60 & 200 & 2007 & 246 & 176.6 & \\
\hline $16: 48$ & $1: 34$ & 56 & 60 & 202 & 2002 & 254 & 182.3 & \\
\hline $16: 50$ & $1: 36$ & 68 & 60 & 202 & 2015 & 246 & 176.6 & \\
\hline $16: 52$ & $1: 38$ & 53 & 60 & 201 & 2002 & 253 & 181.6 & \\
\hline $16: 54$ & $1: 40$ & 69 & 60 & 202 & 2010 & 265 & 190.2 & \\
\hline $16: 56$ & $1: 42$ & 52 & 60 & 202 & 2009 & 252 & 180.9 & \\
\hline $16: 58$ & $1: 44$ & 52 & 60 & 201 & 2005 & 248 & 178.0 & \\
\hline $17: 00$ & $1: 46$ & 55 & 60 & 202 & 2005 & 246 & 176.6 & \\
\hline $17: 02$ & $1: 48$ & 55 & 60 & 203 & 2005 & 247 & 177.3 & \\
\hline $17: 04$ & $1: 50$ & 53 & 60 & 201 & 2002 & 255 & 183.0 & \\
\hline $17: 06$ & $1: 52$ & 60 & 60 & 202 & 2002 & 252 & 180.9 & \\
\hline $17: 08$ & $1: 54$ & 54 & 60 & 202 & 1998 & 254 & 182.3 & \\
\hline $17: 10$ & $1: 56$ & 53 & 60 & 202 & 2002 & 241 & 173.0 & \\
\hline $17: 12$ & $1: 58$ & 52 & 60 & 201 & 2003 & 259 & 185.9 & \\
\hline $17: 14$ & $2: 00$ & 53 & 60 & 202 & 2004 & 253 & 181.6 & \\
\hline $17: 16$ & $2: 02$ & 62 & 60 & 201 & 2017 & 249 & 178.7 & \\
\hline $17: 18$ & $2: 04$ & 68 & 60 & 201 & 2003 & 249 & 178.7 & \\
\hline $17: 20$ & $2: 06$ & 55 & 60 & 201 & 2007 & 255 & 183.0 & \\
\hline $17: 22$ & $2: 08$ & 56 & 60 & 200 & 1999 & 260 & 186.6 & \\
\hline $17: 24$ & $2: 10$ & 55 & 60 & 201 & 2019 & 252 & 180.9 & \\
\hline $17: 26$ & $2: 12$ & 57 & 60 & 201 & 2023 & 252 & 180.9 & \\
\hline $17: 28$ & $2: 14$ & 53 & 60 & 201 & 2003 & 258 & 185.2 & \\
\hline $17: 30$ & $2: 16$ & 57 & 60 & 201 & 2006 & 252 & 180.9 & \\
\hline $17: 32$ & $2: 18$ & 60 & 60 & 201 & 2000 & 253 & 181.6 & \\
\hline $17: 34$ & $2: 20$ & 53 & 60 & 201 & 2011 & 247 & 177.3 & \\
\hline $17: 36$ & $2: 22$ & 57 & 60 & 202 & 2002 & 252 & 180.9 & \\
\hline $17: 38$ & $2: 24$ & 63 & 60 & 201 & 1990 & 248 & 178.0 & \\
\hline $17: 40$ & $2: 26$ & 68 & 60 & 201 & 1996 & 260 & 186.6 & \\
\hline $17: 42$ & $2: 28$ & 53 & 60 & 200 & 2000 & 248 & 178.0 & \\
\hline $17: 44$ & $2: 30$ & 56 & 60 & 200 & 2001 & 241 & 173.0 & \\
\hline $17: 46$ & $2: 32$ & 59 & 60 & 201 & 2000 & 255 & 183.0 & \\
\hline $17: 48$ & $2: 35$ & 51 & 60 & 201 & 2002 & 253 & 181.6 & \\
\hline $17: 50$ & $2: 36$ & 51 & 60 & 200 & 2000 & 243 & 174.4 & \\
\hline $17: 52$ & $2: 39$ & 58 & 60 & 201 & 2019 & 252 & 180.9 & \\
\hline $17: 54$ & $2: 41$ & 52 & 60 & 202 & 2010 & 248 & 178.0 & \\
\hline $17: 56$ & $2: 43$ & 65 & 60 & 201 & 2001 & 250 & 179.5 & \\
\hline $17: 58$ & $2: 45$ & 56 & 60 & 200 & 2002 & 259 & 185.9 & \\
\hline $18: 00$ & $2: 47$ & 69 & 60 & 201 & 2010 & 248 & 178.0 & \\
\hline $18: 02$ & $2: 49$ & 54 & 60 & 201 & 2012 & 245 & 175.9 & \\
\hline $18: 04$ & $2: 51$ & 51 & 60 & 201 & 2000 & 248 & 178.0 & \\
\hline $18: 06$ & $2: 53$ & 57 & 60 & 201 & 1999 & 252 & 180.9 & \\
\hline $18: 08$ & $2: 55$ & 67 & 60 & 201 & 2011 & 256 & 183.8 & \\
\hline $18: 10$ & $2: 57$ & 57 & 60 & 201 & 2002 & 259 & 185.9 & \\
\hline $18: 12$ & $2: 59$ & 64 & 60 & 200 & 2000 & 249 & 178.7 & End Steady-state \\
\hline
\end{tabular}


MATR_27.XLS

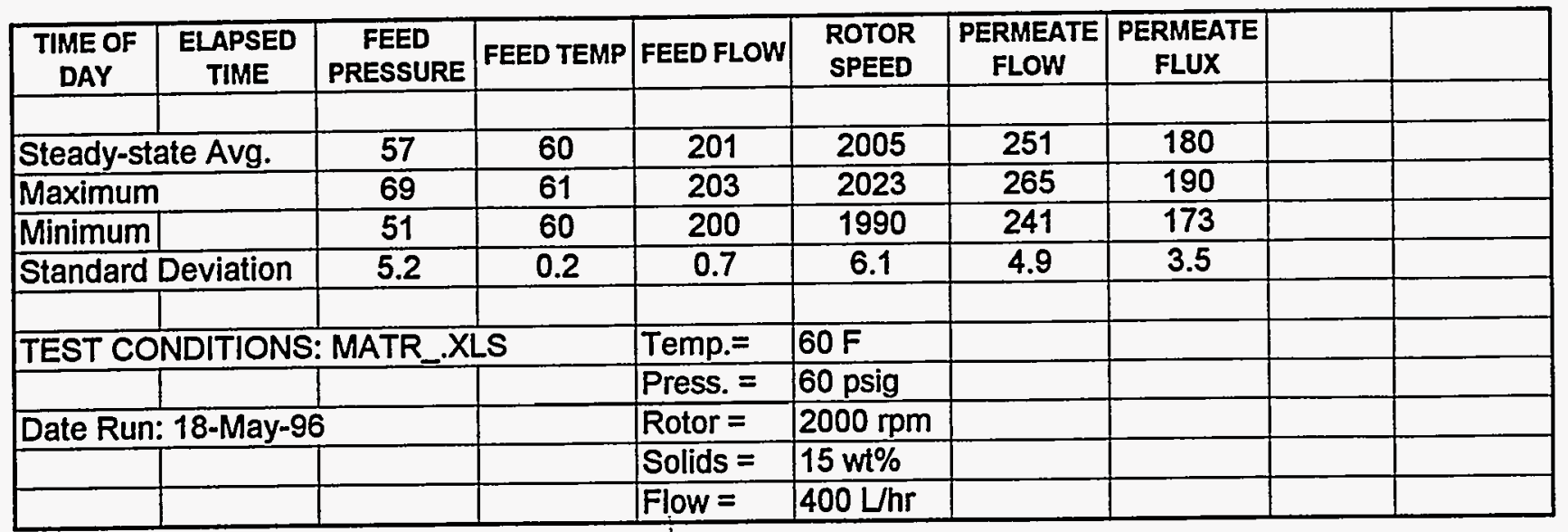

